Dinâmica complexa e formalismo termodinâmico

Carlos Alberto Siqueira Lima 



\title{
Dinâmica complexa e formalismo termodinâmico
}

\author{
Carlos Alberto Siqueira Lima ${ }^{1}$
}

Orientador: Prof. Dr. Daniel Smania Brandão

Dissertação apresentada ao Instituto de Ciências Matemáticas e de Computação - ICMC-USP, como parte dos requisitos para obtenção do título de Mestre em Matemática. VERSÃO REVISADA.

\section{USP - São Carlos \\ Maio/2011}

\footnotetext{
${ }^{1} \mathrm{O}$ autor teve apoio financeiro da FAPESP (N. Processo: 2008/07356-7)
} 
Ficha catalográfica elaborada pela Biblioteca Prof. Achille Bassi e Seção Técnica de Informática, ICMC/USP, com os dados fornecidos pelo(a) autor(a)

\begin{tabular}{|l|} 
S619d Siqueira Lima, Carlos Alberto \\
Dinâmica Complexa e Formalismo Termodinâmico /, \\
Carlos Alberto Siqueira Lima; orientador Daniel \\
Smania Brandão -- São Carlos, 2011. \\
125 p. \\
Dissertação (Mestrado - Programa de Pós-Graduação en \\
Matemática)-- Instituto de Ciências Matemáticas e \\
de Computação, Universidade de São Paulo, 2011. \\
1. Dinâmica Complexa. 2. Teoria Ergódica. 3. \\
Conjuntos de Julia. 4. Dimensão de Hausdorff. I. \\
Smania Brandão, Daniel, orient. II. Título.
\end{tabular}


À Márcia

e Helena. 



\section{Agradecimentos}

Muito tenho aprendido na instituição ICMC-USP, desde 2005, quando iniciava meus estudos de graduação. Encontrei nos Professores a fonte de ideal para o futuro e, nos amigos, companheiros de aprendizado que, sem o saberem, me ajudaram bastante no esforço de "aprender fazendo". Encontrei, também, grandes Mestres, como a Profa. Sueli Mieko T. Aki, que me ensinaram os primeiros passos de uma caminhada, que, por certo, não terá fim. No mestrado renovaram-se as motivações, novos horizontes, fiz novas amizades, e na orientação do Prof. Daniel Smania Brandão recolhi precioso acervo de conhecimento, habilitandome a mais profundos voos dentro da Ciência. Apesar de me reconhecer cada vez. mais ignorante diante do todo, porém muito bem adestrado para lidar com os brinquedos do dia-a-dia, muito tenho a agradecer a todos estes colaboradores. A maioria passará anônima, pois cometi a falta de omitir os nomes daqueles que tiveram influência decisiva na minha vida nos últimos tempos.

Gostaria de agradecer à sociedade que, em seus trabalhos diários, cooperam nos meus estudos. Espero que, nesta pequena obra de esforço pessoal, possa ter correspondido à confiança indireta da multidão, carente do conhecimento que ilumina consciências, incentivadora de pesquisas, e ansiosa pela nossa dedicação na obra educativa.

Agradeço à Fapesp e a todos os Matemáticos, principalmente os da área de Sistemas Dinâmicos e afins, que ajudaram a desenvolver uma teoria tão bela e profunda. 



\section{Resumo}

Estudaremos sistemas dinâmicos complexos da esfera de Riemann, e empregaremos técnicas do Formalismo Termodinâmico - incluindo a fórmula de Bowen - para provar que a dimensão de Hausdorff $\operatorname{dim}_{H} J\left(f_{\lambda}\right)$ do conjunto de Julia $J\left(f_{\lambda}\right)$ de uma família holomorfa de funções racionais hiperbólicas $f_{\lambda}$ define uma função real analítica do parâmetro $\lambda$. Este resultado foi provado por Ruelle [44] em 1981. Daremos uma prova alternativa usando movimentos holomorfos. Trata-se de uma técnica inovadora, originalmente desenvolvida por Mañé, Sad e Sullivan no trabalho [31] sobre estabilidade estrutural de sistemas dinâmicos complexos. 



\section{Abstract}

We shall study complex dynamical systems in the Riemann sphere and prove that the Hausdorff dimension $\operatorname{dim}_{\mathrm{H}} J\left(f_{\lambda}\right)$ of the Julia set $J\left(f_{\lambda}\right)$ of an holomorphic family of hyperbolic rational maps $f_{\lambda}$ defines a real analytic map of the parameter $\lambda$. This result was proved in 1981 by D. Ruelle (see [44]). We give an alternative proof using holomorphic motions (see [31]), which was originally developed to study the structural stability problem of complex dynamical systems. Throughout this work, we shall use several tools of Thermodynamic Formalism, including Bowen's formula. 



\section{Prefácio}

O objetivo deste trabalho é oferecer uma demonstração alternativa de um belo resultado de dinâmica complexa, provado por David Ruelle em 1981. Aproveitamos o ensejo para descrever diversas técnicas da área, dando uma abordagem diferenciada ao tema, visto que parte substancial desta obra é dedicada aos pré-requisitos, não só de dinâmica, mas principalmente os de Análise Complexa em várias variáveis. Grande parte das ferramentas são "importadas", e raramente temos encontrado um material que as reúna num único volume.

A abordagem é, em geral, paciente. Preferimos motivar os conceitos em vez de apresentá-los somente. A única exceção talvez seja o capítulo final, onde fizemos uso implícito de muitas ferramentas descritas nos capítulos precedentes.

Incluímos diversos marcadores (negrito e itálico), principalmente para facilitar a vida dos leitores apressados. O índice, também, está bem detalhado.

Apesar do enfoque ser elementar, existem passagens (raras) que fizemos questão de omitir, por duas razões. A primeira é que elas podem facilmente ser deduzidas - muitas vezes, embora, isso dê algum trabalho -, bastando um nível razoável de compreensão do texto, e um pouco de habilidade com desigualdades. A segunda desculpa é estética e, naturalmente, deve-se ao gosto pessoal do autor.

Subseções e subsubseções serão chamadas apenas de seções. O principal capítulo deste trabalho é o último. De fato, todos os capítulos precedentes são apenas prérequisitos deste. Assim, um modo mais rápido de se apreender o conteúdo total é partir-se diretamente para a leitura do capítulo final, voltando, posteriormente, aos anteriores.

Somos particularmente gratos às sugestões dos membros da banca, constituída pelos Professores Artur Oscar Lopes, Katrin G. Gelfert e Daniel Smania Brandão, que nos possibilitou melhorar a redação final do texto. Reiteramos nossos agradecimentos ao orientador Daniel Smania, afirmando que, desde o momento inicial, foi ele é o verdadeiro idealizador desta obra. Obviamente, coube-nos o trabalho um tanto árduo de organizar todo o conteúdo esparso em mais de 40 referências, imprimindo uma forma original na exposição.

São Carlos, maio de 2011. 



\section{Sumário}

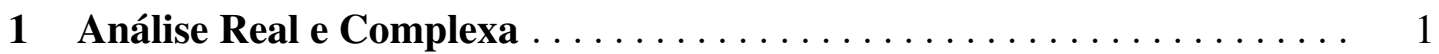

1.1 Teoria da Medida .............................. 3

1.1.1 Regularidade: Medidas de Radon $\ldots \ldots \ldots \ldots \ldots \ldots \ldots \ldots$

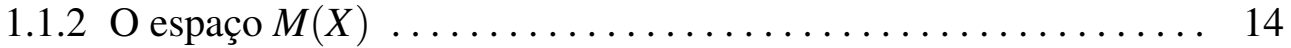

1.2 Medida e Dimensão de Hausdorff $\ldots \ldots \ldots \ldots \ldots \ldots \ldots \ldots \ldots \ldots \ldots . \ldots \ldots$

1.3 O Espaço da funções Hölder contínuas . . . . . . . . . . . . 23

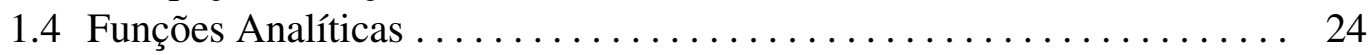

1.4.1 Teorema da função inversa (e implícita) para espaços de Banach . 26

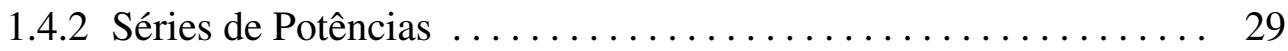

1.4.3 Funções Analíticas .......................... 33

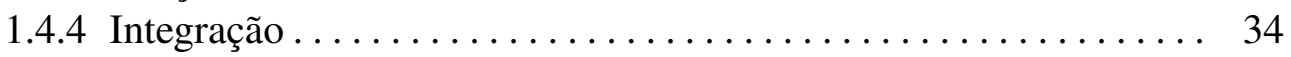

1.4.5 Aplicações Conformes ..................... 45

1.5 Análise Complexa na esfera de Riemann................. 47

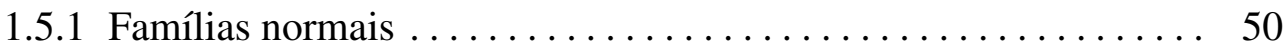

1.5.2 Resultados gerais sobre funções analíticas $\ldots \ldots \ldots \ldots \ldots \ldots 52$

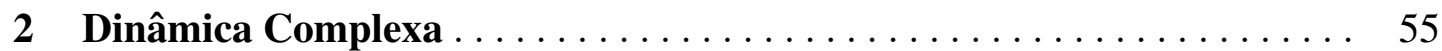

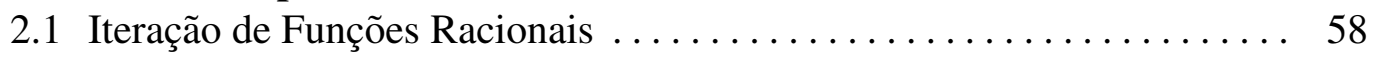

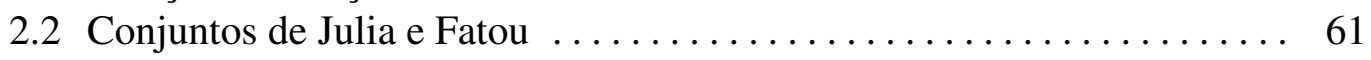

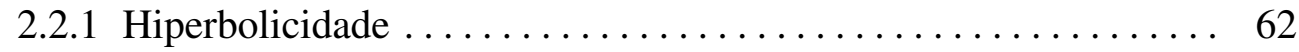

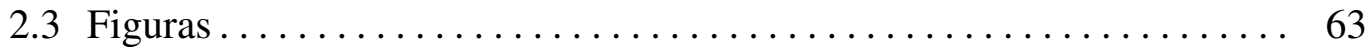

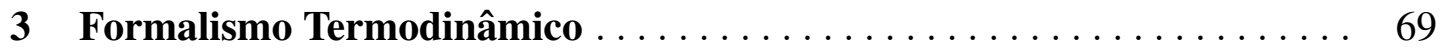

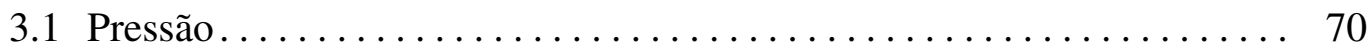

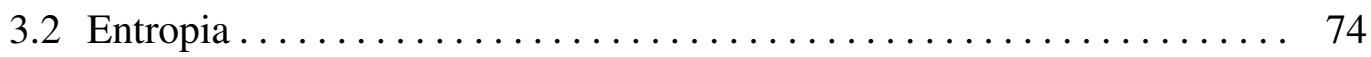

3.3 Transformações expansoras e estados de equilíbrio . . . . . . . . . 79

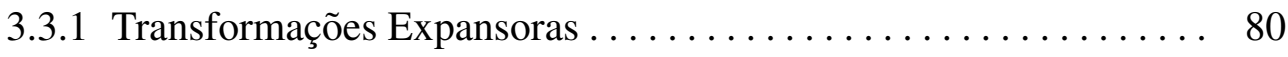

3.3.2 O comportamento do espectro sob perturbação do operador . . . 83

3.3.3 Analiticidade de auto-valores simples . . . . . . . . . . . . 84

3.3.4 Operador de Transferência .................... 85

3.3.5 Definindo pressão para potenciais complexos $\ldots \ldots \ldots \ldots \ldots 87$ 
3.3.6 Cálculo do diferencial da pressão $\ldots \ldots \ldots \ldots \ldots \ldots \ldots \ldots . \ldots 9$

3.3.7 Fórmula de Bowen $\ldots \ldots \ldots \ldots \ldots \ldots \ldots \ldots \ldots \ldots \ldots$

4 Analiticidade real da dimensão de Hausdorff . . . . . . . . . . . . . . 91

4.1 Movimentos holomorfos parametrizados no disco . . . . . . . . . . 91

4.1.1 Extensão de Movimentos holomorfos . . . . . . . . . . . . . . . . 91

4.1.2 Equicontinuidade de movimentos holomorfos ........... 92

$4.2 J$-Estabilidade de funções racionais hiperbólicas . . . . . . . . . 95

4.3 Analiticidade real da dimensão de Hausdorff $\ldots \ldots \ldots \ldots \ldots \ldots \ldots$

Medidas de Haar . . . . . . . . . . . . . . . . . . . . . . . . . . . . . . 109

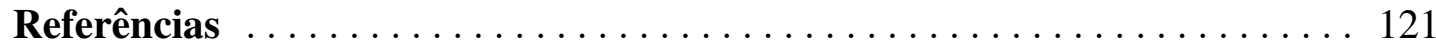

Índice Remissivo . . . . . . . . . . . . . . . . . . . . . . . 123 


\title{
Análise Real e Complexa
}

\begin{abstract}
"Can one learn mathematics by reading it? I am inclined to say no. Reading has an edge over listening because reading is more active - but not much. Reading with pencil and paper on the side is very much better - it is a big step in the right direction. The very best way to read a book, however, with, to be sure, pencil and paper on the side, is to keep the pencil busy on the paper and throw away the book."
\end{abstract}

(PAul Halmos; [23])

Neste capítulo, faremos um estudo preliminar de Análise, incluindo tópicos como Teoria da Medida e Funções Analíticas em espaços normados. Este estudo é de fundamental importância para uma boa compreensão dos próximos capítulos. Procuramos detalhar o índice remissivo, de modo a facilitar eventuais consultas. O leitor familiarizado com o tema pode ir direto ao próximo capítulo e consultar o índice quando necessitar obter informações sobre a notação. Enfatizamos, porém, que o propósito deste capítulo não é meramente informativo; nele, buscamos oferecer ao leitor uma boa visão do assunto, e também demonstramos alguns resultados diretamente relacionados à diferenciabilidade da dimensão de Hausdorff, como o Teorema da Função Implícita em espaços normados e o Lema de Osgood.

\section{Referências: [17] [41] [22] [1] [5] [29] [36] [35].}

\section{Conjuntos e funções}

Denotaremos por $\mathbf{R}$ o corpo dos números reais e por $\mathbf{C}$ o corpo dos números complexos. Usaremos a letra $\emptyset$ para designar o conjunto vazio.

Para $x \in \mathbf{R}$, empregaremos a seguinte nomenclatura:

$$
\begin{cases}x \text { é não-negativo } & \text { se } x \geq 0, \\ x \text { é positivo } & \text { se } x>0, \\ x \text { é não-positivo } & \text { se } x \leq 0 \text { e } \\ x \text { é negativo } & \text { se } x<0 .\end{cases}
$$

Denotaremos o conjunto dos números racionais por $\mathbf{Q}$, os inteiros por $\mathbf{Z}$ e os naturais por $\mathbf{N}$. Notemos que $\mathbf{N}$ coincide com o conjunto dos números inteiros não-negativos. Indicaremos por $\mathbf{R}^{+}$e $\mathbf{Z}^{+}$o conjunto dos números reais positivos e inteiros positivos, respectivamente. 
No contexto de funções de uma ou mais variáveis complexas, será comum denotar um função $f$ por $f(z)$ ou $w=f(z)$. Assim, vemos $z$ e $w$ como variáveis, e $f$ como uma associação unicamente determinada de $z$ para $w$. Aqui, entramos em contato com uma peculiaridade notacional: nem toda notação tem uma conexão direta com o seu fundamento lógico. Por exemplo, o fundamento da expressão sen $z$ como função do plano é o conjunto

$$
\{(z, \operatorname{sen} z): z \in \mathbf{C}\} \text {. }
$$

É claro que, uma vez superado o problema da fundamentação, preferimos lidar com uma linguagem o mais natural possível, longe dos formalismos estéreis.

No caso de conjuntos, usaremos um exemplo para explicar a convenção. Dependendo do caso, poderemos recorrer qualquer uma das expressões

$$
\{z:|z-2|<2\},\{|z-2|<2\} \text { ou }|z-2|<2
$$

para indicar o conjunto dos pontos $z$ do plano que estão a uma distância menor que 2 do ponto 2 .

Um endomorfismo será simplesmente uma aplicação de um conjunto nele mesmo. Usaremos termos como endomorfismo holomorfo, mensurável, etc, cujos significados são óbvios. Um automorfismo é um endomorfismo bijetivo.

Sem maiores explicações, sempre que escrevermos $z=x+i y$ ou $z=\alpha+i \beta$, ficará implícito que os números $x, y, \alpha$ e $\beta$ são reais. A menos que se diga o contrário, ou que estejamos fora do plano complexo, as letras $z$ e $w$ indicarão números complexos, enquanto que $x, y, u, v$ e outras, serão reservadas para as partes real e imaginária. Muitas vezes, escreveremos $f=u+i v$ para denotar uma função complexa de uma variável complexa cujas partes real e imaginária são, respectivamente, $u(z)$ e $v(z)$.

\section{Difinições e Hipóteses}

Não utilizaremos nenhum ambiente especial para Definições e Hipóteses. As definições virão sempre destacadas em negrito, enquanto que as hipóteses serão escritas em itálico, geralmente, no começo de uma seção, ou de um parágrafo. Introduzimos estes marcadores visando facilitar a leitura do texto, e também torná-lo mais fluente, evitando-se a repetição de hipóteses em cada enunciado de uma proposição. Deste modo, muitos resultados serão enunciados admitindo-se tais hipóteses; antes de ler uma Proposição, por exemplo, será conveniente que o leitor procure se inteirar do contexto geral da seção, o que pode ser feito apenas com a leitura dos textos em negrito e itálico.

\section{Parênteses}

Conferimos um certo grau de flexibilidade no uso dos parênteses. Por exemplo, se $V$ for uma vizinhança de um ponto $p$, então denotaremos $V(p)$. Também quebramos a convensão usual de que, ao escrevermos $f(x), f$ será uma função e $x$ a variável. Em alguns casos essa convenção poderá estar presente, mas nem sempre. Por exemplo, 
podemos fixar um ponto $z_{0}$ do plano e à cada parâmetro complexo $\lambda$ atribuir um outro número complexo, denotado por $z_{0}(\lambda)$. De modo algum, $z_{0}$ deixa de ser ponto para se tornar uma função! A função $\lambda \mapsto z_{0}(\lambda)$ será denotada por $z_{0}(\cdot)$, ou simplesmente $z_{0}(\lambda)$.

Ainda no contexto de funções, poderemos omitir o uso dos parênteses. Se $f$ for uma transformação e $x$ for um ponto de seu domínio, então indicaremos por $f x$, ou $f(x)$, o valor de $f$ no ponto $x$, e denotaremos por $f A$, ou $f(A)$, o conjunto dos pontos $f x$, $x \in A$. Se $f_{i}$ for uma transformação de $X_{i}$ em $X_{i+1}$, com $i$ variando de 1 até $n$, então definimos

$$
\left.f_{n} f_{n-1} \cdots f_{1} x:=f_{n}\left(f_{n-1}\left(\cdots f_{2}\left(f_{1}(x)\right)\right)\right) \cdots\right) .
$$

O sinal o de composição de funções algumas vezes será omitido. De três formas diferentes, escreveremos

$$
f g(x)=f \circ g(x)=(f \circ g)(x) .
$$

Essa convenção, porém, pode gerar alguma confusão com relação ao produto de funções. Por exemplo, se $g, f$ forem tais que ambas as operações de composição e multiplicação fazem sentido, então $f g(x)$ será a composta no ponto $x$, enquanto que $(f g)(x)=(f x)(g x)$. De qualquer forma, o contexto não deixará margem a dúvidas.

\subsection{Teoria da Medida}

Seja $X$ um conjunto não vazio. Uma $\sigma$-álgebra de $X$ é uma coleção não-vazia de subconjuntos de $X$, fechada para complementos e reuniões enumeráveis. Diretamente da definição concluímos que ambos $\emptyset$ e $X$ estão em toda $\sigma$-álgebra $\mathscr{A}$ de $X$; e pelas leis de de Morgan, $\mathscr{A}$ também deve ser fechada para interseções enumeráveis. Uma medida sobre $X$ é uma função escalar $\mu$, definida em alguma $\sigma$-álgebra $\mathscr{A}$ de $X$, satisfazendo às propriedades

$$
\mu\left(\bigcup_{i=1}^{\infty} A_{i}\right)=\sum_{i=1}^{\infty} \mu\left(A_{i}\right)
$$

$\mathrm{e}$

$$
\mu(\emptyset)=0,
$$

toda vez que $A_{i}$ for uma coleção enumerável de conjuntos em $\mathscr{A}$ cujos elementos são dois a dois disjuntos. Se $A$ estiver no domínio de $\mu$, então diremos que $A$ é $\mu$-mensurável, ou, simplesmente, mensurável. O contradomínio de uma medida é sempre um dos quatro conjuntos:

$$
\text { (i) } \mathbf{C} ; \quad(i i)(-\infty, \infty] ; \quad(\text { iii })[-\infty, \infty) \text { ou }(\text { iv })[0, \infty]
$$

Naturalmente, quando o contradomínio de $\mu$ for diferente de $[0, \infty)$, devemos exigir que a série em (1.1) seja absolutamente convergente toda vez que a medida da reunião 
$\operatorname{dos} A_{i}$ for finita. Isso porque o lado esquerdo de (1.1) independe da indexação dos conjuntos $A_{i}$, o que faz com que a série seja comutativamente convergente.

Denotaremos o contradomínio de uma medida $\mu$ por $R(\mu)$. Se $R(\mu)=\mathbf{C}$, então diremos que $\mu$ é uma medida complexa. Nos casos em que $R(\mu)$ for um dos conjuntos (ii) ou (iii) entre possibilidades anteriores, diremos que $\mu$ é uma medida com sinal. Não consideramos a possibilidade de $\mu$ assumir ambos os valores $-\infty$ e $\infty$, pois a operação $\infty-\infty$ não está definida. Se $R(\mu)=[0, \infty]$, então diremos que $\mu$ é uma medida positiva. Nessa seção usaremos o termo medida num sentido amplo, incluindo qualquer um dos casos examinados acima. Em futuros tópicos, porém, o leitor deverá ser um pouco cuidadoso, porque vamos nos referir a medidas que são claramente positivas apenas como "medidas", sem nenhuma outra especificação. Essa, aliás, é a convenção usual da literatura.

Uma medida $\mu$ será finita quando seu contradomínio excluir ambos $\infty \mathrm{e}-\infty$; e será $\sigma$-finita quando $X$ puder ser escrito como reunião enumerável de conjuntos $A_{i}$ de medida $\mu$-finita. Podemos ver que as partes real e imaginária de uma medida complexa são medidas finitas com sinal. Por sua vez, toda medida com sinal pode ser expressa como uma diferença de duas medidas positivas. Para tornar esse enunciado mais preciso, faremos a seguinte definição. Sejam $\mu$ e $v$ duas medidas positivas, definidas numa mesma $\sigma$-álgebra de $X$. Diremos que $\mu$ e $v$ são mutuamente singulares - escrevendo $\mu \perp v$ - quando $X$ puder ser escrito como uma reunião disjunta de dois conjuntos mensuráveis $E$ e $F$, com $\mu(F)=0$ e $v(E)=0$. Podemos estender essa definição para medidas com sinal e depois para medidas complexas. Se $\mu$ for uma medida com sinal, um conjunto mensurável $E$ será nulo quando a medida $\mu(F)$ de todo subconjunto mensurável $F \subset E$ for zero. Se $\mu$ e $v$ forem duas medidas com sinal, definidas numa mesma $\sigma$-álgebra de $X$, escreveremos $\mu \perp v$ quando existirem $E$ e $F$, como anteriormente, sendo $E$ nulo para $v$, e $F$ nulo para $\mu$. Já para medidas complexas $\mu$ e $v$, diremos que $\mu \perp v$ quando as partes real e imaginária de $\mu$ forem, cada uma, mutuamente singulares com respeito às partes real e imaginária de $v$.

Teorema 1.1 (Decomposição de Jordan) Toda medida com sinal $\mu$ pode ser unicamente expressa como diferença

$$
\mu=\mu^{+}-\mu^{-}
$$

de duas medidas positivas $\mu^{+}$e $\mu^{-}$- chamadas variação positiva e negativa de $\mu$, respectivamente - ambas definidas na $\sigma$-álgebra de $\mu$, com $\mu^{+} \perp \mu^{-}$.

DEMONSTRAÇÃO. Ver pág. 87 de [17].

A reta real, bem como o plano $\mathbf{C}$, possui uma medida natural, também chamada de medida de Lebesgue, a qual coincide a noção usual de comprimento e área quando aplicada à conjuntos simples, tais como intervalos (caso real), ou triângulos e quadrados (caso complexo). Mais detalhes sobre o assunto podem ser encontrados em quaisquer das referências [17], [22] ou [43]. Por ora, afirmamos que essas medidas estão definidas numa $\sigma$-álgebra que contém a topologia do conjunto. É fácil verificar que 
interseção de $\sigma$-álgebras ainda é uma $\sigma$-álgebra. Definimos a $\sigma$-álgebra de Borel de qualquer espaço topológico, considerando-se a interseção de todas as $\sigma$-álgebras que contém a topologia. Os elementos da $\sigma$-álgebra de Borel são chamados de Borelianos. No caso da medida de Lebesgue (em $\mathbf{R}$ ou $\mathbf{C}$ ), podemos afimar que todo Boreliano é mensurável.

Agora consideremos uma $\sigma$-álgebra em $X$. Diremos que uma função $f$ de $X$ em C $\left(^{1}\right)$ é mensurável com respeito à essa $\sigma$-álgebra quando a imagem inversa $f^{-1}(B)$ de qualquer Boreliano $B$ de $\mathbf{C}$ for um subconjunto mensurável de $X$. Se tivermos uma medida $\mu$ definida nessa $\sigma$-álgebra, então poderemos resumir a definição dizendo simplesmente que $f$ é $\mu$-mensurável. Mas enfatizamos que o conceito de mensurabilidade depende da $\sigma$-álgebra, e não da medida.

Observação. Logo adiante, vamos usar alguns fatos sobre integração de funções mensuráveis com relação a uma medida qualquer. Todos eles podem ser encontrados no Capítulo 2 de [17].

Seja $v$ uma medida com sinal e consideremos uma medida positiva $\mu$, ambas definidas numa mesma $\sigma$-álgebra. Diremos que $v$ é absolutamente contínua com respeito a $\mu$ - escrevendo $v \ll \mu$ - quando $v(E)=0$ toda vez que $\mu(E)=0$. Se $v$ for complexa, $\operatorname{com} \mu$ permanecendo positiva, então escreveremos $v \ll \mu$ quando ambas as partes real e imaginária de $v$ forem absolutamente contínuas com respeito à $\lambda$. Notemos que se $v \perp \mu$ e $v \ll \mu$, então $v=0$. Nesse sentido, ser mutuamente singular está na direção oposta de continuidade absoluta. É natural, portanto, que procuremos decompor uma medida com sinal como soma de outras duas, uma singular e outra absolutamente contínua, tomando-se como referência uma mesma medida positiva (ver Teorema 1.2). Seja $\mu$ uma medida positiva e consideremos uma função $\mu$-mensurável $f$ sobre $X$, com valores na reta real estendida $[-\infty, \infty]$. Diremos que $f$ é $\mu$-integrável no sentido estendido quando alguma das integrais

$$
\int f^{+} d \mu, \quad \int f^{-} d \mu
$$

for finita. Como usual, a integral de uma função integrável no sentido estendido é definida como a soma das duas integrais acima (podendo valer, assim, $-\infty$ ou $\infty$ ).

Sejam $v$ e $\lambda$ medidas definidas numa mesma $\sigma$-álgebra $\mathscr{A}$. Consideremos uma função $\lambda$-mensurável $g$, tomando valores em $\mathbf{R}$ ou $\mathbf{C}$. Se para qualquer conjunto $E \in \mathscr{A}$, tivermos

$$
v(E)=\int_{E} g d \lambda
$$

então escreveremos

$$
d v=g d \lambda
$$

sendo usual que empreguemos quaisquer das notações $d v$ ou $g d \lambda$ para indicar $v$.

\footnotetext{
${ }^{1}$ Mais geralmente, podemos considerar $f$ tomando valores em qualquer espaço topológico $Y$.
} 
Se a medida $\lambda$ acima for positiva, então assumiremos automaticamente que, ao escrevermos $d v=g d \lambda, g$ deverá ser $\lambda$-integrável, ou, pelo menos, integrável no sentido estendido (quando $v$ for uma medida com sinal ou positiva). Um e outro caso ficam determinados pela natureza de $v$ : se $v$ for finita, então $g$ deverá ser integrável com respeito a $\lambda$; e se $v$ não for finita, então este será o caso em que $v$ é uma medida com sinal ou positiva, sendo $g$ uma função a valores em $[-\infty, \infty]$, integrável somente no sentido estendido. Quando $\lambda$ não for positiva, assumiremos sempre que g é integrável com respeito à $\lambda$.

Considerando-se uma medida qualquer $\mu$ sobre $X$, uma propriedade $P$ referente a pontos de $X$ vale $\mu$-quase sempre quando $P$ for verdadeira em todos os pontos de $X$, exceto por um conjunto de medida $\mu$-nula. Usaremos q.s. como forma abreviada de quase sempre. Das propriedades da integral decorre que

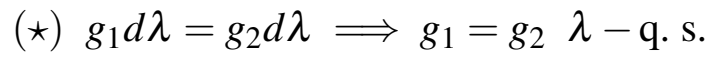

Teorema 1.2 (Lebesgue-Radon-Nikodym) Seja $\mu$ uma medida positiva $\sigma$-finita sobre $X$. Toda medida $\sigma$-finita $v$, definida na mesma $\sigma$-álgebra de $\mu$, pode ser escrita de modo único como uma soma

$$
v=\lambda+\rho
$$

de outras duas medidas $\sigma$-finitas $\lambda, \rho$ do mesmo tipo ${ }^{2}$ de $v$, com $\rho \ll \mu e \lambda \perp \mu$. Além disso, existe $f$ tal que

$$
d \rho=f d \mu .
$$

A função $f$ é única para essa propriedade, no sentido de que qualquer outra g nas mesmas condições deve ser igual a $f \mu$-quase sempre. Se $v$ for positiva, então $f \geq 0$ $\mu$-quase sempre.

DemonstraÇão. Ver [17], págs. 90 e 93.

Como Corolário obtemos um resultado muito interessante, que nos vai permitir definir a derivada de uma medida com relação à outra, sob determinadas condições. Sejam $v$ e $\mu$ nas condições do Teorema anterior. Suponhamos, ainda, que $v$ seja absolutamente contínua com respeito à $\mu$. Então concluímos que $\lambda$ é nula e, portanto, existe uma função $f$, unicamente determinada ( $\mu$-quase sempre), tal que $d v=f d \mu$. Vamos definíla como sendo a derivada de Radon-Nikodym de $v$ com respeito à $\mu$, escrevendo

$$
f=\frac{d v}{d \mu}
$$

Trata-se, portanto, de uma classe de equivalência de funções, na qual duas funções mensuráveis estão indenficadas se, e somente se, elas coincidem $\mu$-quase sempre sobre $X$. Na prática, trataremos essas classes pelos seus representantes, tomando-se apenas o cuidado de que uma igualdade de funções, nesse contexto, significa somente uma igualdade válida $\mu$-quase sempre. Logo, veremos a derivada de Radon-Nikodym como

\footnotetext{
2"Tipo", aqui, se refere aos termos: complexa, positiva, com sinal.
} 
uma função, a qual (pelas observações que precedem o Teorema anterior) deverá ser $\mu$-integrável quando $v$ for finita; e integrável no sentido estendido quando $v$ for uma medida real - positiva ou com sinal.

As propriedades de cancelamento

$$
g d v=g \frac{d v}{d \mu} d \mu
$$

$$
\frac{d v}{d \lambda}=\frac{d v}{d \mu} \frac{d \mu}{d \lambda}(\lambda \text {-q.s. })
$$

sugeridas pela notação, são válidas, mas mediante as seguintes condições, facilmente memorizadas pelo fato de que elas são exigências naturais para que as igualdades acima façam sentido. A primeira delas é que todas as medidas em questão devem ser $\sigma$-finitas, com

$$
v \ll \lambda, \quad v \ll \mu \ll \lambda .
$$

Em (1.4), $g$ não pode ser qualquer função mensurável; devemos supor que ela seja integrável com respeito a $v$. Sob essa condição, verifica-se que $g(d \nu / d \mu)$ é integrável com respeito à $\mu$, valendo (1.4). Estes resultados estão todos demonstrados em [17], págs. 90 e 91.

Agora usaremos a derivada de Radon-Nikodym para definir a variação total de uma medida $v$. Se $v$ for uma medida positiva, então $|v|$ será a própria $v$. Se $v$ for uma medida com sinal, então definiremos $|v|$ como sendo a soma de suas variações positiva e negativa. Observemos que, nesses dois casos, $|v|$ é uma medida positiva, com relação a qual $v$ é absolutamente contínua. Essa propriedade ainda será válida para o caso complexo, que definiremos logo mais. Suponhamos, então, que $v$ seja uma medida complexa; seja $\mu$ a soma das quatro medidas positivas que determinam $v$, isto é, as variações positiva e negativa de suas partes real e imaginária. Observamos, então, que $v \ll \mu$. Logo, existe uma função $\mu$-integrável $g$, a valores complexos, tal que

$$
d v=g d \mu
$$

e definimos

$$
d|v|=|g| d \mu .
$$

O modo pelo qual definimos $|v|$ não deixa espaço para ambiguidades; todavia, afirmamos que $|v|$ é independente da escolha de $\mu$, desde que tomemos $\mu$ positiva, com $v \ll \mu$. Com efeito, sejam $\mu_{1}, \mu_{2}$ duas medidas positivas nessas condições. Consideremos funções $f_{1}$ e $f_{2}$, integráveis com respeito a $\mu_{1}$ e $\mu_{2}$, respectivamente, com

$$
f_{1} d \mu_{1}=f_{2} d \mu_{2}
$$

Então introduzimos $\lambda=\mu_{1}+\mu_{2}$, e usando (1.4), escrevemos (1.8) na forma 


$$
f_{1} \frac{d \mu_{1}}{d \lambda} d \lambda=f_{2} \frac{d \mu_{2}}{d \lambda} d \lambda
$$

de onde concluímos que os dois integrandos são iguais $\lambda$-quase sempre. Portanto, o mesmo vale para seus módulos; e como as derivadas $d \mu_{i} / d \lambda$ são positivas, podemos escrever

$$
\left|f_{1}\right| \frac{d \mu_{1}}{d \lambda}=\left|f_{2}\right| \frac{d \mu_{2}}{d \lambda}
$$

Essa última igualdade pode ser colocada na forma desejada $\left|f_{1}\right| d \mu_{1}=\left|f_{2}\right| d \mu_{2}$, bastando que se "multiplique" $d \lambda$ em ambos os lados [ver (1.4)].

Observação. A definição de $|v|$ por meio de integrais não vale apenas para o caso em que $v$ é complexa, mas também nos demais. Por exemplo, se $v$ for uma medida com sinal, escolhemos ${ }^{3} f=1_{E^{+}}-1_{E^{-}}$(onde $E^{+}$e $E^{-}$são os dois conjuntos $v$-mensuráveis determinados pela decomposição de Jordan), e observamos que $|v|=v^{+}+v^{-}$verifica

$$
d v=f d\left(v^{+}+v^{-}\right), d|v|=|f| d\left(v^{+}+v^{-}\right) .
$$

A variação total de uma medida $v$ está definida na mesma $\sigma$-álgebra de $v$. Se $E$ for um conjunto $v$-mensurável, então, de acordo com a notação anterior,

$$
|v(E)|=\left|\int_{E} f d \mu\right| \leq \int_{E}|f| d \mu=|v|(E) ;
$$

o que mostra que $v$ é absolutamente contínua com respeito a $|v|$. Podemos ver que o módulo da derivada de $v$ com relação à sua variação total é igual a 1 . Para tanto, colocamos $\lambda=|v|$ e escrevemos $v$ na forma (1.3), com $g=d v / d|v|$; em seguida, aplicamos a definição de variação total e da propriedade $(\star)$ vemos que $|g|=1 \lambda$-q.s. Finalmente, observamos que uma função $f$ será $v$-integrável se, e somente se, também for integrável com relação à sua variação total, valendo

$$
\left|\int f d v\right| \leq \int|f| d|v|,
$$

sempre que $f$ for $v$-integrável (ver [17], p. 94). Com relação a desigualdade acima, lembramos que $d \mu$ comporta-se de forma linear na variável $\mu$, de modo que se $v_{R}$ e $v_{I}$ forem as partes real e imaginária de $v$ (quando esta for complexa), então

$$
\int f d v=\int f d v_{R}+i \int f d v_{I}
$$

\footnotetext{
${ }^{3} 1_{A}(x)$, ou $\chi_{A}(x)$, é a função característica do conjunto $A$, ou seja, vale 1 quando $x$ estiver em $A$ e 0 caso contrário.
} 


\subsubsection{Regularidade: Medidas de Radon}

Agora vamos explorar o conceito de medida em espaços topológicos, analisando medidas positivas sob o aspecto contínuo, ou seja, estudaremos propriedades de aproximação advindas da topologia. Restringiremos nossos estudos ao caso das medidas positivas; voltaremos a considerar o caso geral na próxima seção. Assumiremos que X é um espaço topológico localmente compacto e Hausdorff. Logo,

$(\star \star)$ para toda vizinhança $V(p)$ existe uma outra vizinhança $U(p)$, cujo fecho é um compacto contido em $V$.

Essa propriedade - e outras como Lema de Urysohn, etc. - será de fundamental importância nos resultados que daremos a seguir. Nosso enfoque, todavia, não será sistemático; e em vista disso, muitas das idéias essenciais ficarão apenas implícitas nos enunciados. Todo o conteúdo foi extraído de [17].

Denotaremos por $\mathscr{B}(X)$ a $\sigma$-álgebra dos Borelianos de $X$; por $\mathscr{F}_{\sigma}(X)$ a classe dos Borelianos de $X$ que podem ser escritos como reunião enumerável de fechados; e por $\mathscr{G}_{\delta}(X)$ a coleção de todos os Borelianos de $X$ que podem ser expressos como o interseção enumerável de abertos. Omitiremos o espaço $X$, sempre que não houver confusão. Se $\mu$ for uma medida definida em $\mathscr{B}(X)$, então diremos que $\mu$ é uma medida de Borel - ou Boreliana - de $X$.

Dados um Boreliano $E$ de $X$ e uma medida de Borel $\mu$ sobre $X$, diremos que $\mu$ é exteriormente regular sobre $E$ quando $\mu(E)$ for o ínfimo das medidas dos abertos que contém $E$. Diremos, ainda, que $\mu$ é internamente regular sobre $E$ se $\mu(E)$ for o supremo das medidas dos compactos que estão contidos em $E$. As medidas de Borel sobre $X$ que forem exteriormente regulares sobre qualquer Boreliano, internamente regulares sobre todo aberto e finitas sobre compactos serão chamadas de Medidas de Radon. Assim, vemos que uma medida de Radon é unicamente determinada pelos seus valores sobre os subconjuntos compactos.

Proposição 1.1 Se $\mu$ for uma medida de Radon $\sigma$-finita sobre $X$, então, para qualquer Boreliano E de X:

(1) Para todo $\varepsilon>0$, existem um fechado $F$ contido em $E$ e um aberto $U$ contendo $E$ tais que $\mu(U \backslash F)<\varepsilon$;

(2) Existem um $A \in \mathscr{F}_{\sigma}$ contido em $E$ e um $B \in \mathscr{G}_{\delta}$ contendo $E$ tais que $\mu(B \backslash A)=0$.

Existem muitos exemplos de medidas de Radon. O próximo resultado, por exemplo, afirma que se $X$ for segundo enumerável, então qualquer medida de Borel $\mu$ finita sobre compactos será uma medida de Radon. Este resultado será de extrema valia no futuro, onde estudaremos medidas finitas sobre espaços métricos compactos, em conexão com o transposto do operador de Ruelle-Perron-Frobenius. Por ora, apenas lembramos que todo espaço métrico compacto é segundo enumerável.

Para o critério que daremos a seguir, precisaremos de um outro conceito. Uma medida Boreliana $\mu$ é chamada de regular quando $\mu$ é interna e externamente regular 
sobre qualquer Boreliano. Um subconjunto $E$ de $X$ será $\sigma$-compacto quando $E$ for uma reunião enumerável de compactos. Notemos que se $X$ for segundo enumerável, então todo aberto de $X$ será $\sigma$-compacto [basta considerar uma base local satisfazendo $(\star \star)]$.

Teorema 1.3 (Regularidade) Se todo aberto de $X$ for $\sigma$-compacto, então qualquer medida Boreliana $\mu$ sobre $X$ que for finita sobre compactos será regular e, portanto, de Radon. Em particular, se X tiver uma base enumerável, então toda medida Boreliana finita sobre compactos será regular.

DEMONSTRAÇÃO. Ver final da seção.

Observamos que um resultado semelhante vale para espaços métricos (não necessariamente localmente compactos):

Teorema 1.4 (Regularidade) Seja $M$ um espaço métrico, no qual todo fechado é $\sigma$-compacto. Então toda medida Boreliana finita $\mu$ sobre $M$ é regular.

DemonstraÇão. Seja $\mathscr{R}$ a classe dos Borelianos de $M$ sobre os quais $\mu$ é regular. Mostraremos que $\mathscr{R}$ é uma $\sigma$-álgebra que contém todos os fechados de $M$. Primeiramente, verifiquemos que $\mathscr{R}$ é fechada para reuniões enumeráveis. Seja $\left\{A_{n}\right\}_{1}^{\infty}$ uma subclasse de $\mathscr{R}$, e seja $A$ sua reunião. Consideremos um $\varepsilon>0$ arbitrário. Vamos construir uma conjunto aberto $U$ contendo $A$ e outro compacto $C$ contido em $A$ tais que $\mu(U \backslash C)<\varepsilon$. Isso é equivalente à condição de regularidade de $\mu \mathrm{em} A$. Para cada $n$ existem um aberto $U_{n}$ contendo $A_{n}$ e um compacto $C_{n}$ contido em $A_{n}$ tais que $\mu\left(U_{n} \backslash C_{n}\right)<\varepsilon / 3^{n}$. Tomamos, então, $U$ como sendo a reunião dos $U_{n}$, definimos $\check{C}$ como sendo a reunião $\operatorname{dos} C_{n}$, e colocamos

$$
C:=\bigcup_{n=1}^{k} C_{n},
$$

onde $k$ é escolhido de forma que $\mu(\check{C} \backslash C)<\varepsilon / 2$ (aqui usamos o fato de que o espaço é de medida finita). $\mathrm{O}$ compacto $C$ está contido em $A$, que por sua vez está contido no aberto $U$. Além disso,

$$
\begin{aligned}
\mu(U \backslash C) & \leq \mu(U \backslash \check{C})+\mu(\check{C} \backslash C) \\
& \leq \sum_{n=1}^{\infty} \mu\left(U_{n} \backslash C_{n}\right)+\mu(\check{C} \backslash C) \\
& <\sum_{n=1}^{\infty} \frac{\varepsilon}{3^{n}}+\frac{\varepsilon}{2}=\varepsilon .
\end{aligned}
$$

Usando-se o mesmo argumento acima pode-se mostrar que $\mathscr{R}$ é fechada para complementos. Para verificar que todo subconjunto fechado $F$ de $M$ está em $\mathscr{R}$, basta considerar os abertos 


$$
U_{n}=\{x \in M: d(x, F)<1 / n\},
$$

e usar o fato de que $F$ pode ser escrito como uma reunião enumerável de compactos encaixados.

Agora enunciaremos algumas propriedades de aproximação para funções mensuráveis. Muitas outras, como Teorema da Convergência Dominada, etc, podem ser encontradas em [17].

Usaremos $1_{A}$ ou $\chi_{A}$ para denotar a função característica de um conjunto qualquer $A$. Recordemos que $1_{A}$ vale 1 sobre $A$, e zero no seu complementar. Em nível crescente de complexidade, depois das funções características temos as funções simples, que nada mais são que combinações lineares finitas (sobre $\mathbf{C}$ ) de funções características. Logo, nenhuma função simples pode assumir qualquer dos valores $\infty,-\infty$. Vamos considerar apenas funções simples que sejam mensuráveis. Obviamente, uma função simples pode ser escrita de formas diferentes como combinação linear de funções características. Contudo, existe uma maneira independente de se tratá-las: funções simples são aquelas a cuja imagem consiste de um número finito de valores $a_{j}$, todos diferentes de $\infty,-\infty$. Assim, toda função simples possui uma representação canônica, dada como combinação linear das funções $\chi_{f^{-1} a_{j}}$, as quais serão mensuráveis precisamente quando a função simples for mensurável.

Sem fazer menção à qualquer aspecto relacionado à topologia do conjunto, podemos afirmar que qualquer função mensurável sobre $X$ pode ser aproximada por funções simples:

Proposição 1.2 (Aproximação por funções simples) Seja $Y$ um conjunto não-vazio e consideremos uma $\sigma$-álgebra de $Y$. Se f for uma função mensurável sobre $Y$, com valores em $\mathbf{R} \cup\{\infty\}$ ou $\mathbf{C}$, então existirá uma sequência de funções mensuráveis simples $\phi_{n}$ tais que

$$
0 \leq\left|\phi_{1}\right| \leq\left|\phi_{2}\right| \leq \cdots \leq|f|, \quad \phi_{n} \rightarrow f \text { (pontualmente) }
$$

A convergência acima será uniforme sobre todo conjunto onde $f$ for limitada. Além disso, se $f \geq 0$, então poderemos escolher $\phi_{n} \geq 0$, satisfazendo às mesmas propriedades acima.

Demonstração. Ver [17], pág. 47.

Consideremos os espaços de Banach $\left(\mathscr{L}^{p}(\mu),\|\cdot\|_{p}\right), 1 \leq p \leq \infty$. Para $p \neq \infty, \mathscr{L}^{p}$ constitui-se das funções mensuráveis $f: X \rightarrow \mathbf{C}$ (identificadas quando coincidirem $\mu$-q.s.) tais que $|f|^{p}$ é integrável, com

$$
\|f\|_{p}=\left(\int|f|^{p} d \mu\right)^{1 / p} .
$$

Já $\mathscr{L}^{\infty}(\mu)$ é o espaço de todas as funções $\mu$-mensuráveis $f$ que são limitadas $\mu$-quase sempre, ou seja, $|f| \leq M$, num conjunto de medida total, para algum $M$. O ínfimo das constantes $M$, para uma dada função $f$, é chamadado de supremo essencial, o qual denota-se por 


$$
\|f\|_{\infty}=\sup _{x \in X} \operatorname{ess}|f(x)| .
$$

A função $\|\cdot\|_{\infty}$ é a norma do espaço $\mathscr{L}^{\infty}$, com relação à qual ele é completo. Para detalhes, ver [17], Cap. 6.

Considerando-se as funções simples que estão em $\mathscr{L}^{p}(\mu), p \in[1, \infty)$, podemos afirmar que o fecho deste conjunto na norma $\|\cdot\|_{p}$ é todo o espaço $\mathscr{L}^{p}(\mu)$ (ver [17], págs. 183,184). Esse é um resultado que vale mesmo quando $\mu$ não for uma medida de Radon; de fato, $X$ sequer precisa ser um espaço topológico. Mas se $\mu$ for Radon, então valem resultados mais fortes.

Teorema 1.5 Seja $\mu$ uma medida de Radon sobre $X$. O conjunto $C_{c}(X)$ das funções contínuas e de suporte compacto é denso em $\left(\mathscr{L}^{p}(\mu),\|\cdot\|_{p}\right)$, para $1 \leq p<\infty$.

DEMONSTRAÇÃo. Ver [17], pág. 217.

Dada qualquer $f$, definimos $\|f\|_{u}$ como sendo o supremo do módulo $|f|$ sobre $X$.

Teorema 1.6 (Lusin) Seja $\mu$ uma medida de Radon sobre X. Dados $\varepsilon>0$ e uma função mensurável

$$
f: X \rightarrow \mathbf{C},
$$

existe $\phi \in C_{c}(X)$ tal que $f=\phi$ fora de um conjunto de medida $\mu$ menor que $\varepsilon$. Podemos tomar $\phi$ de modo que $\|\phi\|_{u}$ seja menor ou igual que $\|f\|_{u}$.

DEMONSTRAÇÃO. Ver [17], pág. 217.

Agora enunciaremos o Teorema da Representação da Riesz para medidas de Radon, um poderoso ingrediente na construção de medidas invariantes, sendo, portanto, uma ferramenta de fundamental importância em Teoria Ergódica.

Um funcional linear

$$
I: C_{c}(X) \rightarrow \mathbf{C}
$$

será positivo quando $I(f) \geq 0$ toda vez que $f$ for real e $f \geq 0$. Tais funcionais serão sempre limitados sobre compactos $K \subset X$, no sentido de que se $I$ for positivo, então necessariamente existirá uma constante $C_{K}$ tal que

$$
|I(f)| \leq C_{K}\left(\sup _{x \in X}|f(x)|\right)
$$

para toda $f \in C_{c}(X)$ cujo suporte esteja contido em $K$ (ver [17], pág. 212).

No próximo resultado, usaremos a seguinte notação. Se $U$ for um aberto de $X$, designaremos por $\underline{A}(U)$ o conjunto de todas as funções $f \in C_{c}(X)$ cujo suporte está contido em $U$ e que satisfazem $0 \leq f \leq 1$. Se $K$ for um compacto, então

$$
\bar{A}(K)=\left\{f \in C_{c}(X) ; f \geq 1_{K}\right\}
$$


Teorema 1.7 (Representação de Riesz) A cada funcional linear positivo I sobre $C_{c}(X)$ corresponde uma única medida de Radon $\mu$ sobre $X$ tal que

$$
I(f)=\int f d \mu
$$

para toda $f \in C_{c}(X)$. Além disso, para qualquer aberto $U$ e qualquer compacto $K$,

$$
\mu(U)=\sup _{f \in \underline{A}(U)} I(f) \quad \text { e } \mu(K)=\inf _{f \in \bar{A}(K)} I(f) .
$$

DEMONSTRAÇÃO. Ver [17], pág. 213.

Como toda medida de Radon $\mu$ é finita sobre compactos, a expressão (1.13) sempre define um funcional linear positivo $I_{\mu}$ sobre $C_{c}(X)$. O Teorema da Representação de Riesz estabelece uma correspondência bijetora $\mu \mapsto I_{\mu}$ entre o conjunto das medidas de Radon e o conjunto dos funcionais lineares positivos sobre $C_{c}(X)$.

Com o auxílio do Teorema da Representação, faremos a

DEMONSTRAÇÃo Do TEOREMA 1.3 - Como $\mu$ é finita sobre compactos, toda função contínua de suporte compacto é $\mu$-integrável. Com isso, podemos afirmar que $I_{\mu}(f)$ está bem definido para $f \in C_{c}(X)$, sendo um funcional linear positivo. Usando o Teorema anterior, vemos que existe uma medida de Radon $v$ sobre $X$ tal que

$$
\int f d \mu=\int f d v, f \in C_{c}(X)
$$

Mostraremos que $\mu$ e $v$ são iguais. Consideremos, primeiramente, um aberto qualquer $U$, que, por hipótese, escreve-se como reunião enumerável de compactos $K_{j}$. Com o auxílio do Lema de Urysohn (ver [17], pág. 131), construímos uma sequência $f_{j}$ em $C_{c}(X)$, definindo o primeiro termo $f_{1}$ de forma que seu suporte esteja contido em $U$ e $\left.f_{1}\right|_{K_{1}}=1_{K_{1}}$; indutivamente, tomamos $0 \leq f_{n} \leq 1$, com suporte ainda contido em $U$, com a propriedade de que, quando restrita à reunião dos compactos

$$
K_{1} \cup \cdots \cup K_{n} \cup \operatorname{supp}\left(f_{1}\right) \cup \cdots \cup \operatorname{supp}\left(f_{n-1}\right),
$$

$f_{n}$ vale 1 . Podemos ver que $f_{j}$ converge pontualmente para $\chi_{U}$ e, além disso, $f_{j} \leq f_{j+1}$. Do Teorema da Convergência Monótona (cf. [17], pág. 50) concluímos que

$$
\mu(U)=\int \chi_{U} d \mu=\lim _{n \rightarrow \infty} \int f_{n} d \mu=\lim _{n \rightarrow \infty} \int f_{n} d v=v(U) .
$$

Logo, $\mu$ e $v$ coincidem sobre abertos. Seja $E$ um Boreliano qualquer. Dado $\varepsilon>0$, existem um aberto $V$ contendo $E$ e um fechado $F$ contido em $E$ tais que $v(V \backslash F)<\varepsilon$; de modo que

$$
\mu(V)-\mu(E) \leq \mu(V \backslash F)=v(V \backslash F)<\varepsilon
$$

e 


$$
\mu(E)-\mu(F) \leq \mu(V \backslash F)<\varepsilon .
$$

Como $F$ é $\sigma$-compacto, existem compactos $K_{j}$ tais que $\mu\left(K_{j}\right) \rightarrow \mu(F)$. Logo, existe um compacto $K \subset E$ tal que $\mu(E) \leq \mu(K)+\varepsilon$. Com estes fatos, concluímos que $\mu$ é interior e exteriormente regular sobre qualquer Boreliano $E$; em particular, $\mu$ é uma medida de Radon, e pela unicidade garantida pelo Teorema de Riesz, temos $\mu=v$.

\subsubsection{O espaço $M(X)$}

Consideremos os espaço de Banach $C_{0}(X)$, constituído das funções complexas e contínuas definidas em $X$ que se anulam no infinito, ${ }^{4}$ munido da norma uniforme

$$
\|f\|_{u}=\sup _{x \in X}|f(x)|
$$

Objetivamos identificar o dual contínuo $C_{0}(X)^{*}$ com o conjunto $M(X)$ de todas as medidas complexas definidas na $\sigma$-álgebra de Borel de $X$. Naturalmente, usaremos o Teorema da Representação de Riesz para estabelecer uma bijeção entre estes dois conjuntos. Vamos supor que $X$ é um espaço topológico segundo enumerável, localmente compacto e Hausdorff.

As partes real e imaginária de $\mu \in M(X)$ são medidas finitas com sinal, cujas variações positiva e negativa são medidas positivas regulares sobre $X$, pelo Teorema 1.3. Assim, todas as medidas $\mu \in M(X)$ são regulares. Essa, aliás, é uma consequência que simplificará bastante a nossa exposição. (Nossa principal aplicação será dada em espaços métricos compactos). Apesar disso, observamos que os resultados dessa seção se estendem de modo natural para espaços que sejam apenas localmente compactos e Hausdorff, sendo $M(X)$ substituído pelo conjunto das medidas Borelianas complexas de Radon. Para essa abordagem - um pouco mais geral - consultar [17], págs. $221 \mathrm{e}$ seguintes.

Começaremos definindo uma norma em $M(X)$. Se $\mu \in M(X)$, então colocamos

$$
\|\mu\|=|\mu|(X) .
$$

Proposição $1.3(M(X),\|\cdot\|)$ é um espaço vetorial normado sobre $\mathbf{C}$, com as operações usuais de soma e multiplicação por escalar.

Demonstração. Seja $\mu$ em $M(X)$. Por (1.11), temos $\mu \ll|\mu|$, o que faz com que $\mu=0$ toda vez que $\|\mu\|=0$. Da definição de variação total segue-se que

$$
\|c \mu\|=|c|\|\mu\|
$$

Falta mostrar a desigualdade triangular. Se $\mu_{1}, \mu_{2}$ estiverem em $M(X)$, definimos $\lambda$ como sendo a soma das variações totais $\left|\mu_{1}\right|,\left|\mu_{2}\right|$; e escrevemos

$$
d \mu_{1}=f_{1} d \lambda, d \mu_{2}=f_{2} d \lambda .
$$

\footnotetext{
${ }^{4}$ Em termos precisos: $f \in C_{0}(X)$ se, e somente se, dado $\varepsilon>0$, existir um compacto $K \subset X$, fora do qual $|f|<\varepsilon$.
} 
Como

$$
d\left(\mu_{1}+\mu_{2}\right)=d \mu_{1}+d \mu_{2}=\left(f_{1}+f_{2}\right) d \lambda,
$$

obtemos

$$
d\left|\mu_{1}+\mu_{2}\right|=\left|f_{1}+f_{2}\right| d \lambda \leq\left|f_{1}\right| d \lambda+\left|f_{2}\right| d \lambda=d\left|\mu_{1}\right|+d\left|\mu_{2}\right|
$$

de onde segue a desigualdade triangular.

Como $X$ é localmente compacto e Hausdorff, $C_{0}(X)$ é o fecho de $C_{c}(X)$ na norma uniforme $\|\cdot\|_{u}$ (ver [17], pág. 132). Esse fato nos permitirá aplicar o Teorema da Representação de Riesz.

Se $\mu$ for uma medida Boreliana positiva e finita sobre $X$, então $I_{\mu}$ - ver o comentário feito logo depois do Teorema 1.7 - define um funcional linear positivo sobre $C_{0}(X)$, com

$$
\left|I_{\mu}(f)\right| \leq \mu(X)\|f\|_{u} ;
$$

de onde segue que $I_{\mu}$ é limitado. Por outro lado, se $I$ for um funcional linear positivo sobre $C_{0}(X)$ - em particular, positivo, como funcional linear de $C_{c}(X)$ - então existirá uma única medida de Radon positiva sobre $X$ tal que $I=I_{\mu}$ sobre $C_{c}(X)$. (Observemos que, como estamos supondo $X$ segundo enumerável, a classe das medidas de Radon coincide com a classe de todas as medidas finitas sobre compactos; por sua vez, todas essas medidas serão não apenas Radon, mas também regulares. Assim, a afirmação de unicidade vale para todas das medidas em questão). Logo, $I_{\mu}$ é um funcional linear limitado sobre $C_{c}(X)$, o que acarreta $\mu(X)<\infty$, pela propriedade (1.14). Portanto, toda função de $C_{0}(X)$ é $\mu$-integrável e $I_{\mu}$ está definido em todo o espaço $C_{0}(X)$. A igualdade $I=I_{\mu}$ se extende continuamente de $C_{c}(X)$ para o fecho $C_{0}(X)$. Assim:

(*) Para todo funcional linear limitado e positivo I sobre $C_{0}(X)$, existe uma única medida Boreliana positiva e finita $\mu$ sobre $X$ tal que $I=I_{\mu}$. Reciprocamente, $I_{\mu}$ será um funcional linear positivo e limitado sobre $C_{0}(X)$ toda vez que $\mu$ for uma medida Boreliana finita e positiva de X.

Para lidar com funcionais lineares mais gerais, precisamos desenvolver um meio de decompô-los como diferença de funcionais lineares positivos. A abordadem será mais simples se, primeiramente, considerarmos funcionais reais.

Seja $I$ um funcional linear, real e limitado, definido no conjunto $C_{0, R}(X)$, constituído de todas as $f \in C_{0}(X)$ cujos valores são reais.

Lema 1.1 Existem funcionais lineares limitados, positivos e reais $I^{+}, I^{-}$sobre $C_{0, R}(X)$, tais que $I=I^{+}-I^{-}$.

Demonstração. Precisamos determinar $I^{+}$e $I^{-}$a partir de $I$. Para tanto, vamos explorar algumas consequências de uma decomposição deste tipo, supondo-se que ela exista. Obteremos relações que nos indicarão como definir $I^{+}$e $I^{-}$convenientemente. Como $I^{-}$é positivo, devemos ter $I^{+}(f) \geq I(f)$, sempre que $f \geq 0$. Mas $I^{+}$também é positivo; logo,

$$
I(g) \leq I^{+}(g)=-I^{+}(f-g)+I^{+}(f) \leq I^{+}(f),
$$


toda vez que $0 \leq g \leq f$. Quando $g=0$, as desigualdades acima nos dirão que $I^{+}(f) \geq 0$. Tendo-se em vista essas propridades, definimos $I^{+}(f)$, para $f \geq 0$, como sendo o supremo dos valores $I(g)$, tomado sobre todas as funções $g \in C_{0, R}(X)$ satisfazendo $0 \leq g \leq f$. Para $f, h \geq 0$ e $c \in \mathbf{R}, c \geq 0$, temos

$$
\begin{gathered}
0 \leq I^{+}(f) \leq\|I\|\|f\|_{u} ; \\
I^{+}(c f)=c I^{+}(f) ; \\
I^{+}(f)+I^{+}(h) \leq I^{+}(f+h) .
\end{gathered}
$$

Podemos provar o "outro lado" de (1.17). Com efeito, para toda $g \in C_{0, R}(X)$ satisfazendo $0 \leq g \leq f+h$, encontramos outros dois elementos $g_{1}, g_{2}$ de $C_{0, R}(X)$ com

$$
g=g_{1}+g_{2} ; 0 \leq g_{1} \leq f ; \quad 0 \leq g_{2} \leq h .
$$

(Dica: reduza as condições envolvendo $g_{1}$ e $g_{2}$ em um conjunto de condições envolvendo apenas $g_{1}$; encontre uma solução e depois coloque $\left.g_{2}=g-g_{1}\right)$. Tomando-se o supremo sobre todas as possíveis $g$, obtemos a desigualdade oposta de (1.17); de onde

$$
I^{+}(f+h)=I^{+}(f)+I^{+}(h), \quad(f, h \geq 0) .
$$

Agora estendemos linearmente $I^{+}(f)$ para qualquer $f \in C_{0, R}(X)$, pondo

$$
I^{+}(f)=I^{+}\left(f^{+}\right)-I^{+}\left(f^{-}\right) .
$$

Se $f=f^{+}-f^{-}=g-h$, com $g, h \geq 0$, então teremos $g+f^{-}=h+f^{+}$; e usando (1.18), encontramos

$$
I^{+}(f)=I^{+}(g)-I^{+}(h) .
$$

Decompondo-se $f$ como diferença das funções positivas $f^{+}$e $f^{-}$, concluímos da igualdade acima e de (1.16) que $I^{+}$é linear sobre $C_{0, R}(X)$. A mesma decomposição também nos mostra que $I^{+}$é limitado. Pelo modo como definimos $I^{+}$, a diferença $I^{-}=I-I^{+}$ é um funcional linear positivo e limitado sobre $C_{0, R}(X)$.

Notemos que, para todo $\mu \in M(X)$, o funcional $I_{\mu}$ está definido em todo o espaço $C_{0}(X)$. Usando-se (1.12), vemos que $I_{\mu}$ é limitado, com $\left\|I_{\mu}\right\| \leq\|\mu\|$. Podemos aplicar o Lema anterior para mostrar que a aplicação linear $\mu \mapsto I_{\mu}$, de $M(X)$ em $C_{0}(X)^{*}$, é sobrejetiva. De fato, todo $f \in C_{0}(X)$ decompõe-se na sua parte real e imaginária $u, v$, respectivamente; de modo que se $I \in C_{0}(X)^{*}$, então $I(f)=I(u)+i I(v)$. Agora observemos que $I(u)$ é um funcional linear na variável real $u \in C_{0, R}(X)$, cujos valores são complexos; logo,

$$
I(u)=P(u)+i Q(u),
$$

onde $P$ e $Q$ são funcionais lineares limitados, reais e definidos no espaço $C_{0, R}(X)$. Usamos o Lema anterior para decompor $P$ como diferença de funcionais lineares limitados, positivos e reais $P^{+}, P^{-}$, definidos em $C_{0, R}(X)$, os quais podem ser estendidos linearmente para o espaço complexo $C_{0}(X)$, dando origem a outros dois funcionais 
lineares limitados e positivos sobre $C_{0}(X)$, que continuaremos denotando por $P^{+} \mathrm{e}$ $P^{-}$. O Teorema da Representação de Riesz aplica-se à este caso, e de $(\star)$ concluímos que existem medidas positivas e finitas $\mu_{1}, \mu_{2}$ em $M(X)$ tais que $P^{+}=I_{\mu_{1}}$ e $P^{-}=I_{\mu_{2}}$ sobre $C_{0}(X)$. Consequentemente,

$$
P=I_{\mu_{1}}-I_{\mu_{2}}
$$

e, por argumentos idênticos,

$$
Q=I_{\mu_{3}}-I_{\mu_{4}}
$$

para medidas positivas $\mu_{3}, \mu_{4}$ satisfazendo às mesmas propriedades. Obtemos, assim, uma decomposição para $I(u)$, a qual também vale para $I(v)$; e depois de algumas manipulações, encontramos $I=I_{\mu}$, onde

$$
\mu=\mu_{1}-\mu_{2}+i\left(\mu_{3}-\mu_{4}\right) .
$$

Teorema 1.8 (Representação de Riesz) A função $\mu \mapsto I_{\mu}$ é uma bijeção linear de $M(X)$ sobre $C_{0}(X)^{*}$, com

$$
\left\|I_{\mu}\right\|=\|\mu\| .
$$

Demonstração. A igualdade (1.19) nos diz que a correspondência $\mu \mapsto I_{\mu}$ é uma isometria linear, o que faz com que essa aplicação seja automaticamente injetora. Deste modo, tudo que precisamos mostrar é que $\left\|I_{\mu}\right\| \geq\|\mu\|$, pois os outros fatos já foram provados - ou são consequências imediatas deste. Já sabemos que $\mu \ll|\mu|$ [cf. (1.11)], e que a função $h=d \mu / d|\mu|$ tem valor absoluto constante e igual a 1 . Dado $\varepsilon>0$, usamos o Teorema de Lusin para encontrar uma função contínua $\phi \in C_{c}(X)$, com $\|\phi\|_{u} \leq 1$ e $\phi=\bar{h}$ sobre $X$, exceto por um conjunto $|\mu|$-mensurável $E$, com $|\mu|(E)<\varepsilon$. Assim,

$$
\begin{aligned}
\|\mu\| & =\int_{X} \bar{h} h d|\mu|=\int \bar{h} \frac{d \mu}{d|\mu|} d|\mu| \\
& =\int \bar{h} d \mu=\left|\int(\bar{h}-\phi+\phi) d \mu\right| \\
& \leq 2|\mu|(E)+\left|I_{\mu}(\phi)\right| \\
& \leq 2 \varepsilon+\left\|I_{\mu}\right\|,
\end{aligned}
$$

como queríamos demonstrar.

\subsection{Medida e Dimensão de Hausdorff}

Nosso objetivo é estender o conceito de dimensão, familiar no estudo de variedades diferenciáveis, para objetos fragmentados, como os conjuntos de Julia e, mais geralmente, os Fractais. Daremos um enfoque analítico ao tema, já que visamos apenas definir dimensão de Hausdorff em espaços métricos. Um estudo mais aprofundado, com exemplos enriquecedores, é dado em [15], onde o autor utiliza o conceito 
de dimensão de Hausdorff como ferramenta no estudo de Fractais. No decorrer das argumentações, usaremos alguns fatos sobre medidas de Haar, os quais estão descritos no Apêndice.

Comecemos, pois, com alguns conceitos básicos. Consideremos um espaço métrico $(X, d)$. Uma medida exterior $\mu^{*}$ sobre $X$ será chamada de medida exterior métrica toda vez que $\mu^{*}$ for aditiva sobre conjuntos que estão a uma distância positiva um do outro, i. é.,

$$
\mu^{*}(A \cup B)=\mu^{*}(A)+\mu^{*}(B),
$$

sempre que $d(A, B)>0$. Neste ponto, recordamos o Teorema de Carathéodory [cf. [17], pág. 29], que nos diz que o conjunto dos $\mu^{*}$-mensuráveis forma uma $\sigma$-álgebra, e que a restrição da medida exterior à estes conjuntos nos dá uma medida completa. No caso de uma medida exterior métrica, qualquer Boreliano será um elemento da $\sigma$-álgebra dos $\mu^{*}$-mensuráveis, como afirma a próxima

Proposição 1.4 Se $\mu^{*}$ for uma medida exterior métrica sobre um espaço métrico $X$, então qualquer Boreliano será $\mu^{*}$-mensurável.

DEMONSTRAÇÃo. Será suficiente mostrar que todo fechado $F$ é $\mu^{*}$-mensurável, pois os conjuntos fechados geram a $\sigma$-álgebra de Borel. Mostraremos, então, que para todo subconjunto $A$ de $X$,

$$
\mu^{*}(A) \geq \mu^{*}(A \cap F)+\mu^{*}(A \backslash F) .
$$

Podemos supor que $\mu^{*}(A)<\infty$, caso contrário, a desigualdade anterior será trivialmente satisfeita. Os fechados $B_{n}=\left\{x \in A \backslash F: d(x, F) \geq n^{-1}\right\}$ estão encaixados e contidos em $A \backslash F$. Por outro lado, se $x \in A \backslash F$, então teremos $d(x, F)>0$, já que $F$ é fechado. Deste modo, $B_{n}$ é uma sequência encaixada de conjuntos cuja reunião é $A \backslash F$. E como

$$
\mu^{*}(A) \geq \mu^{*}\left((A \cap F) \cup B_{n}\right)=\mu^{*}(A \cap F)+\mu^{*}\left(B_{n}\right),
$$

devemos mostrar apenas que $\mu^{*}\left(B_{n}\right) \rightarrow \mu^{*}(A \backslash F)$. Escrevemos $C_{n}=B_{n+1} \backslash B_{n}$, para $n \geq 1$, e afirmamos que

$$
d\left(C_{n+1}, B_{n}\right) \geq \frac{1}{n(n+1)},
$$

pois se $x \in C_{n+1}$ e $d(x, y)<[n(n+1)]^{-1}$, então

$$
d(y, F) \leq d(x, y)+d(x, F)<\frac{1}{n(n+1)}+\frac{1}{n+1}=\frac{1}{n} ;
$$

e concluímos daí que se $y$ estiver em $B_{n}$, então $d(x, y)<[n(n+1)]^{-1}$ não poderá ser satisfeita. Como $C_{n-1} \subset B_{n}$, em particular temos $d\left(C_{n+1}, C_{n-1}\right)>0$, o que nos garante a aditividade de $\mu^{*}$ para os conjuntos $C_{n+1}$ e $C_{n-1}$. Assim,

$$
\sum_{1}^{n} \mu^{*}\left(C_{2 j}\right)=\mu^{*}\left(\bigcup_{1}^{n} C_{2 j}\right) \leq \mu^{*}(A)<\infty
$$




$$
\sum_{1}^{n} \mu^{*}\left(C_{2 j-1}\right)=\mu^{*}\left(\bigcup_{1}^{n} C_{2 j-1}\right) \leq \mu^{*}(A)<\infty .
$$

Portanto, a série cujos termos são $\mu^{*}\left(C_{j}\right)$ converge, fazendo com que os restos $\sum_{n+1}^{\infty} \mu^{*}\left(C_{j}\right)$ convirjam para zero. Como $\mu^{*}$ é subaditiva, temos

$$
\mu^{*}(A \backslash F) \leq \mu^{*}\left(B_{n}\right)+\sum_{1}^{\infty} \mu^{*}\left(C_{j}\right)
$$

de onde segue $\mu^{*}(A \backslash F) \leq \lim \inf \mu^{*}\left(B_{n}\right) \leq \lim \sup \mu^{*}\left(B_{n}\right) \leq \mu^{*}(A \backslash F)$, como queríamos mostrar.

Agora definiremos uma medida exterior métrica que dará origem às medidas de Hausdorff de dimensão fragmentada, ou seja, queremos associar à cada número real $s \geq 0$ uma medida exterior $h_{s}^{*}$ que seja boa para medir subconjuntos de dimensão $s$. O termo dimensão ainda carece de definição formal. Nas próximas linhas, buscaremos motivar a definição que daremos deste conceito.

Mudemos o ambiente de $X$ para $\mathbf{R}^{n}$, e denotemos por $\mu$ a medida de Lebesgue deste espaço. Fixado um Boreliano $F$, $\operatorname{sabemos}^{5}$ que para todo $t \geq 0$,

$$
\mu(t F)=t^{n} \mu(F) \text { e }|t F|^{n}=t^{n}|F|,
$$

de modo que o quociente $\mu(t F) /|t F|$ é sempre constante. De fato, considerando-se a clase $\mathscr{C}$ de todos os Borelianos que são imagem de $F$ por uma homotetia, isometria, ou compostas sucessivas destas aplicações, existe um número $\alpha$ dependendo somente de $\mathscr{C}$ tal que $\mu(C)=\alpha|C|^{n}$, para todo $C$ em $\mathscr{C}$. O que faremos é ignorar o fator de normalização $\alpha$, que depende apenas da "forma" do conjunto, e definir uma medida exterior levando-se em consideração apenas o diâmetro. Neste ponto da discussão, retornaremos ao contexto abstrato.

Dados $s \geq 0$ e $\delta>0$, para qualquer subconjunto $A$ de $X$ definimos

$$
h_{s, \delta}^{*}(A)=\inf \left\{\sum_{j=1}^{\infty}\left|C_{j}\right|^{s}: A \subset \bigcup_{j=1}^{\infty} C_{j} \text { e }\left|C_{j}\right| \leq \delta\right\} .
$$

(O conjunto acima pode inclusive ser vazio; em todo caso, $\inf \emptyset=\infty$ ). Os conjuntos $C_{j}$ podem ser substituídos por abertos, sem que o valor de $h_{s, \delta}^{*}$ se altere, bastando que se substitua $C_{j}$ pelo aberto $U_{j}=\left\{x: d\left(x, C_{j}\right)<\varepsilon 2^{-j-1}\right\}$, cujo diâmetro é menor que $\left|C_{j}\right|+\varepsilon 2^{-j-1}$. Quando $\delta \rightarrow 0$, os valores de $h_{s, \delta}^{*}$ crescem monotonamente e, assim, podemos considerar o limite

$$
\lim _{\delta \rightarrow 0} h_{s, \delta}^{*}(A),
$$

o qual denotamos por $h_{s}^{*}(A)$ e definimos como sendo a medida (exterior) $s$-dimensional de Hausdorff do conjunto $A$. Antes que provemos que $h_{s}^{*}$ é de fato uma medida

\footnotetext{
${ }^{5}$ Denotaremos o diâmetro de um conjunto $C$ por $|C|$.
} 
exterior, convém esclarecer o leitor o porque de tomarmos refinamentos sucessivos da cobertura na definição de $h_{s}^{*}$. Umas das justificativas é a seguinte: queremos medir conjuntos fragmentados e, para isso, devemos definir um objeto leve em consideração detalhes do conjunto em escalas cada vez menores. Não precisamos ir muito longe para exibir um exemplo. O leitor poderá analisar o gráfico da função $y=\operatorname{sen}(1 / x)$ e ver que o ínfimo das somas em (1.20) sobre todas as coberturas difere do limite quando $\delta \rightarrow 0$. Agora mostremos que $h_{s}^{*}$ é uma medida exterior. Sabemos que cada $h_{s, \delta}^{*}$ é uma medida exterior, pois, de um modo mais geral,

Proposição 1.5 Se E for uma família de subconjuntos de um conjunto qualquer $Y$, com $\emptyset, Y \in E$, então qualquer função $\rho: E \rightarrow[0, \infty]$ que satisfaça $\rho(\emptyset)=0$ induz uma medida exterior $v^{*}$ sobre $Y$ através de

$$
v^{*}(A)=\inf \left\{\sum_{1}^{\infty} \rho\left(A_{j}\right): A_{j} \in E, A \subset \bigcup A_{j}\right\} .
$$

Demonstração. Ver [17], Capítulo 1.

Como $h_{s}^{*}$ é definida como o limite de medidas exteriores, segue-se que $h_{s}^{*}$ também é exterior. Por outro lado, se $A$ e $B$ forem dois subconjuntos quaisquer de $X$ tais que $d(A, B)>0$, então, tomando-se $\delta<d(A, B)$, concluímos que

$$
h_{s, \delta}^{*}(A \cup B) \geq h_{s, \delta}^{*}(A)+h_{s, \delta}^{*}(B) ;
$$

$\operatorname{logo}, h_{s}^{*}(A \cup B)=h_{s}^{*}(A)+h_{s}^{*}(B)$. Provamos, assim, que cada $h_{s}^{*}$ é uma medida exterior sobre $X$, e que todo Boreliano é mensurável. A restrição de $h_{s}^{*}$ à $\sigma$-álgebra de Borel nos fornece, portanto, uma medida de Borel sobre $X$, que denotaremos por $h_{s}$. Essa é a medida de Hausdorff $s$-dimensional. Sempre que nos referirmos à tal medida, ficará entendido que se trata da medida de Borel, e não da medida completa dada pelo Teorema de Carathéodoy. Mais adiante, veremos que $h_{n}$, à menos de um fator constante, coincide com a medida de Lebesgue sobre $\mathbf{R}^{n}$ (ver Teo. 1.9).

Se $X$ for um espaço vetorial sobre $\mathbf{R}$, então segue direto da definição que

Proposição 1.6 Para todo Boreliano A de X e qualquer $t \geq 0, h_{s}(t A)=t^{S} h_{S}(A)$.

Do ponto de vista formal, o que fizemos foi apenas definir um conjunto de medidas Borelianas $h_{s}$. Para introduzirmos o conceito de dimensão, observamos que, fixado $A$, a função $s \mapsto h_{s}(A)$ é não-crescente com $s$. Além disso, se a medida $h_{s}(A)$ for finita, então para qualquer dimensão maior $t>s$, a correspondente medida $h_{t}(A)$ será nula, pois, de fato, para qualquer cobertura $\left\{U_{i}\right\}$ de $A$ por conjuntos $U_{i}$ cujo diâmetro é menor que $\delta$, tem-se

$$
\sum_{i}\left|U_{i}\right|^{t} \leq \delta^{t-s} \sum_{i}\left|U_{i}\right|^{s} \longrightarrow 0, \text { quando } \delta \rightarrow 0 .
$$

A função $s \mapsto h_{s}(A)$ possui, então, um único ponto de descontinuidade $s_{0}$; se $s_{0}>0$, então este ponto corresponde ao "salto" de $\infty$ para zero. Definimos $s_{0}$ como sendo 
a dimensão de Hausdorff de $A$, denotada por $\operatorname{dim}_{\mathrm{H}} A$. Seguindo a motivação dada no início desta seção, deveremos mostrar que a dimensão de Hausdorff dos espaços Euclidianos coincide com a noção usual de dimensão para espaços vetoriais. De fato,

Teorema 1.9 Existe uma constante $c_{n}>0$ tal que $c_{n} h_{n}$ é a medida de Lebesgue sobre $\mathbf{R}^{n}$. Em particular, a dimensão de Hausdorff de $\mathbf{R}^{n}$ é $n$.

DEMONSTRAÇÃo. Segue da própria definição que $h_{n}$ é uma medida de Borel invariante por translações. Seja $\mu$ a medida de Lebesgue em $\mathbf{R}^{n}$. Mostraremos que $h_{n}$ é uma medida de Haar e, pela unicidade de medidas de Haar - a menos de constante - seguirá o resultado. $\mathbf{R}^{n}$ é um espaço de Hausdorff localmente compacto em que todo aberto é $\sigma$-compacto. Para mostrar que $h_{n}$ é Radon - e portanto, Haar tanto à esquerda quanto à direita (ver Apêndice) - devemos verificar que $h_{s}$ é finita sobre compactos. Também mostraremos que $h_{n}$ é não-nula, para podermos garantir que $c_{n}>0$. Consideremos o quadrado $Q=[0,1]^{n}$. Dado $\varepsilon>0$, escolhemos um natural $k>1$ tal que $2^{-k} \sqrt{n}<\varepsilon$. Dividimos $[0,1] \mathrm{em} 2^{k}$ subintervalos e, com isso, escrevemos $Q$ como reunião de $N=2^{\text {nk }}$ "cubos" $C_{j}, \operatorname{com}\left|C_{j}\right|=2^{-k} \sqrt{n}$. Como

$$
h_{n, \varepsilon}(Q) \leq \sum_{1}^{N}\left|C_{j}\right|^{n} \leq 2^{n k} 2^{-k n}(\sqrt{n})^{n}=(\sqrt{n})^{n},
$$

devemos ter $h_{n}(Q)<\infty$. Agora mostraremos que $h_{n}(Q)>0$. Para $a \in \mathbf{R}^{n}$, consideremos os conjuntos

$$
\bar{B}_{e}(a, r)=\left\{x \in \mathbf{R}^{n}: \sum_{1}^{n}\left|x_{i}-a_{i}\right|^{2} \leq r\right\} ; \bar{B}_{\text {max }}(a, r)=\left\{x \in \mathbf{R}^{n}:\left|x_{i}-a_{i}\right| \leq r\right\} .
$$

Para subconjuntos $E$ de diâmetro $d$ finito, temos $E \subset \bar{B}_{e}(a, 2 d) \subset \bar{B}_{\text {max }}(a, 2 d)$, qualquer que seja $a \in E$; de modo que $\mu(E) \leq(4 d)^{n}=4^{n}|E|^{n}$. Portanto, para toda cobertura aberta $\left\{A_{j}\right\}$ de $Q$, com $\left|A_{j}\right| \leq \varepsilon$, temos $\left|A_{j}\right|^{n} \geq \mu(A) 4^{-n}$. Conluímos então que $h_{n, \varepsilon}^{*}(Q)$ é maior que o ínfimo dos valores $\mu(A) 4^{-n}$, com $A$ variando entre todos os abertos que contém $Q$. Como a medida de Lebesque $\mu$ é regular, segue daí que $h_{n, \varepsilon}^{*}(Q) \geq$ $\mu(Q) 4^{-n}=4^{-n}>0$, como queríamos.

Veremos agora que aplicações bi-Lipschitz preservam dimensão de Hausdorff de conjuntos. Consideremos o caso mais geral de uma função Hölder contínua (ver próxima seção) $f$ de $A \subset X$ em $X$, com expoente $\alpha$, onde $A$ é qualquer subconjunto de $X$. Segue diretamente da definição de $h_{s}^{*}$ que para todo $B \subset A$ (não necessariamente mensurável)

$$
h_{s / \alpha}^{*}(f B) \leq c^{s / \alpha} h_{s}^{*}(B)
$$

de modo que

$$
\operatorname{dim}_{\mathrm{H}} f(B) \leq(1 / \alpha) \operatorname{dim}_{\mathrm{H}} B,
$$

toda vez que $B$ e $f(B)$ forem mensuráveis. 
Quando $f$ for Lipschitziana, o valor de $\alpha$ será 1, e a última desigualdade nos diz que a dimensão da imagem de um conjunto não excede a sua própria dimensão. No caso de aplicações bi-Lipschitz, teremos

Proposição 1.7 Seja $f$ uma função bi-Lipschitz de um conjunto qualquer $A \subset X$ em $X$, ou seja,

$$
C_{1} d(x, y) \leq d(f(x), f(y)) \leq C_{2} d(x, y),
$$

então a imagem $f(B)$ de qualquer Boreliano $B \subset X$ é outro Borelino de mesma dimensão que $B: \operatorname{dim}_{\mathrm{H}} B=\operatorname{dim}_{\mathrm{H}} f(B)$.

Existem diversas técnicas para se calcular a dimensão de Hausdorff, variando das mais simples às mais elaboradas. Os dois exemplos que apresentaremos enquadram-se na primeira categoria e podem ser encontrados em [15], pág 31. Faremos uma descrição um tanto superficial. O conjunto ternário de Cantor $C \subset \mathbf{R}$, um compacto de medida nula (Lebesgue de $\mathbf{R}$ ) mas que, apesar disso, possui a mesma cardinalidade da reta, sendo também totalmente disconexo e perfeito. Sua dimensão de Hausdorff é $\delta=\log 2 / \log 3=0.6309 \ldots, \operatorname{com} 1 / 2 \leq h_{\delta}(C) \leq 1$. Outro exemplo é o triangulo de Sierpiński, obtido de uma construção iterativa parecida com a do conjunto ternário de Cantor, a qual indicamos na seguinte sequência de figuras: ${ }^{6}$
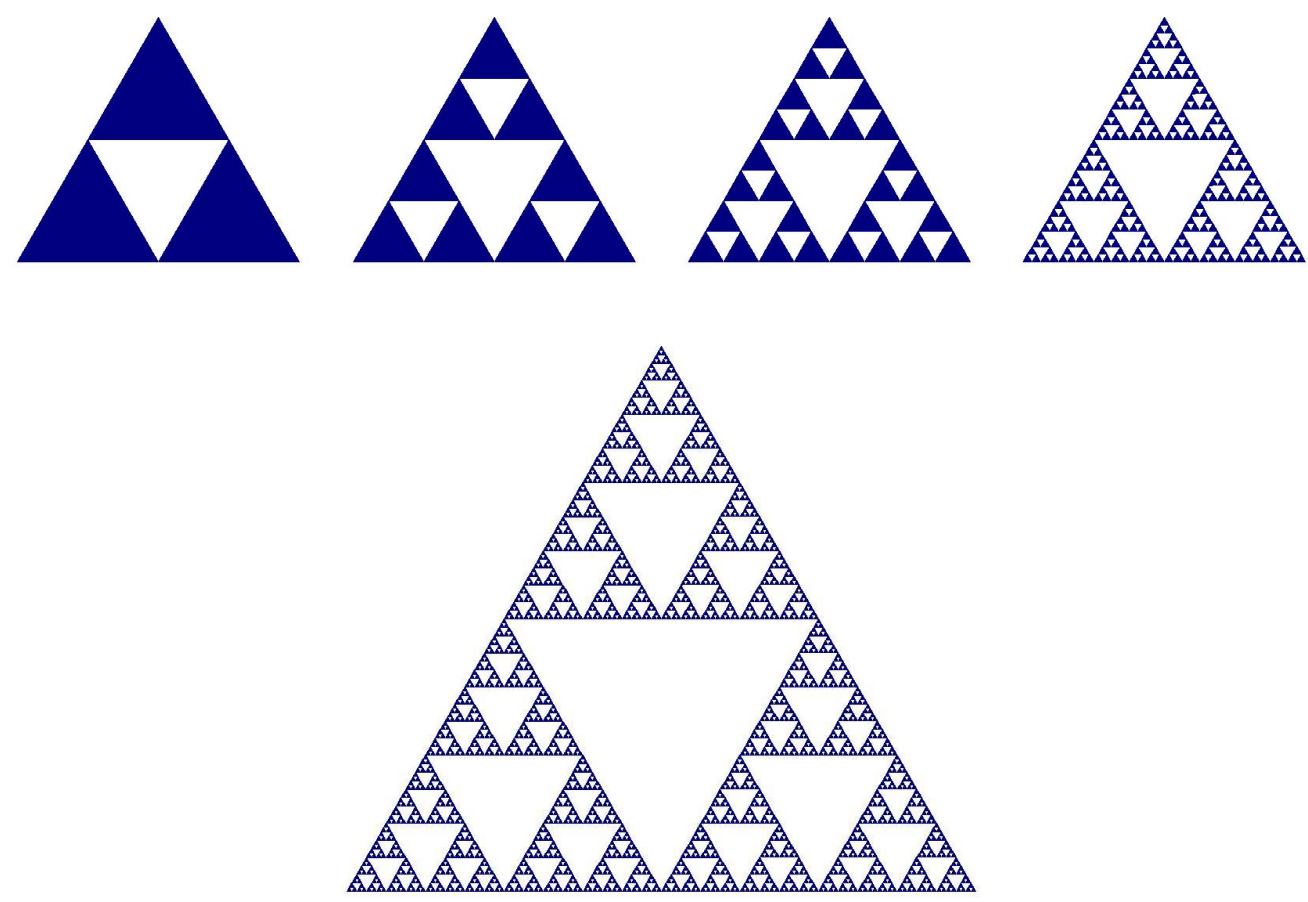

A formulação matemática dessa construção é evidente e a deixamos a cargo do leitor. Nas figuras acima esboçamos (em azul) $E_{1}, E_{2}, E_{3}, E_{5}$ e $E_{7}$, respectivamente, onde $E_{k}$ é o $k$-ésimo conjunto obtido nesse processo. O triângulo de Sierpiński é a interseção de todos os $E_{k}$ 's; sua dimensão de Hausdorff é $\log 3 / \log 2$.

\footnotetext{
${ }^{6}$ Todas foram feitas com o Software disponível em http://www.efg2.com/Lab/.
} 


\subsection{O Espaço da funções Hölder contínuas}

Seja X um espaço métrico compacto e $\gamma$ um número real no intervalo $(0,1]$. Uma função contínua $f$, definida em $X$ e tomando valores em $\mathbf{C}$, será chamada de Hölder contínua com expoente $\gamma$ quando existir uma constante $C \geq 0$ tal que

$$
|f(x)-f(y)| \leq C d(x, y)^{\gamma},
$$

para quaisquer $x, y \in X$. Qualquer $C \geq 0$ que verifique a desigualdade acima é chamada de constante de Hölder de $f$. A ínfimo de todas as constantes de Hölder de $f$ será denotado por $\mathrm{C}_{\mathrm{H}}(f)$. É imediato que

$$
\mathrm{C}_{\mathrm{H}}(f)=\sup _{d(x, y)>0} \frac{|f(x)-f(y)|}{d(x, y)^{\gamma}} .
$$

Logo, $\mathrm{C}_{\mathrm{H}}(f)$ também é uma constante de Hölder de $f$. O conjunto de todas as funções complexas e Hölder contínuas com expoente $\gamma$ será denotado por $C^{\gamma}(X)$. Este é um espaço vetorial sobre $\mathbf{C}$, com as operações usuais de soma e produto por escalar. Também está bem definido o produto de funções neste conjunto. É fácil verificar que $C^{\gamma}(X)$ é uma álgebra sobre C. (Ver definição de álgebra em [25], pág. 126). Tendo em vista (1.22), definimos

$$
\mathrm{C}_{\mathrm{H}, \delta}(f):=\sup _{0<d(x, y)<\delta} \frac{|f(x)-f(y)|}{d(x, y)^{\gamma}},
$$

para toda $f \in C^{\gamma}(X)$. Como

$$
\mathrm{C}_{\mathrm{H}, \delta}(f+g) \leq \mathrm{C}_{\mathrm{H}, \delta}(f)+\mathrm{C}_{\mathrm{H}, \delta}(g) \text { e } \mathrm{C}_{\mathrm{H}, \delta}(\lambda f)=|\lambda| \mathrm{C}_{\mathrm{H}, \delta}(f),
$$

a igualdade

$$
\|f\|_{\gamma, \delta}=\|f\|_{\infty}+\mathrm{C}_{\mathrm{H}, \delta}(f)
$$

define uma norma sobre $C^{\gamma}(X)$. Recordemos que $\|f\|_{\infty}$ é o supremo de $|f|$ sobre $X$.

Proposição 1.8 $C^{\gamma}(X)$ é um espaço de Banach com a norma $\|\cdot\|_{\gamma, \delta}$.

Demonstração. Sabemos que o conjunto $C(X)$ de todas as funções complexas e contínuas de $X$ é um espaço de Banach com a norma $\|\cdot\|_{\infty} ; \operatorname{logo}$, toda sequência de Cauchy $f_{n}$ em $C^{\gamma}(X)$ deve convergir uniformemente para uma função $f \in C(X)$. Pela primeira desigualdade em (1.23), temos uma cota superior para todos os números $\mathrm{C}_{\mathrm{H}}\left(f_{n}\right)$, o que acarreta $f \in C^{\gamma}(X)$. Resta-nos mostrar que $\left\|f_{n}-f\right\|_{\gamma, \delta}$ converge para zero. Isso segue da seguinte desigualdade

$$
\frac{\left|\left(f_{n}-f\right)(x)-\left(f_{n}-f\right)(y)\right|}{d(x, y)^{\gamma}} \leq C_{\mathrm{H}, \delta}\left(f_{n}-f_{m}\right)+\frac{2\left\|f_{m}-f\right\|_{\infty}}{d(x, y)^{\gamma}} .
$$

Para simplificar a notação, omitiremos a constante $\delta$ da norma $\|\cdot\|_{\gamma, \delta}$. O valor específico de $\delta$ não influi em grande parte dos argumentos. 
Proposição 1.9 $C^{\gamma}(X)$ é denso em $\left(C(X),\|\cdot\|_{\infty}\right)$.

DEMONSTRAÇÃo. Esta é uma consequência natural do Teorema de Stone-Weierstrass (para um enunciado deste Teorema, ver [25], pág. 129). Tudo que precisamos mostrar é que $C^{\gamma}(X)$ é completamente separante, ou seja, que dados quaisquer dois pontos distintos $x$ e $y$ de $X$, existem funções $f$ e $g$ em $C^{\gamma}(X)$ tais que $f(x) \neq f(y)$ e $f(x) \neq 0$. Para tanto, é suficiente provar que, independentemente dos valores de $f\left(x_{0}\right)$ e $C \geq 0$, a igualdade

$$
f(x)=f\left(x_{0}\right)+C d\left(x, x_{0}\right)^{\gamma}
$$

define uma função Hölder contínua com expoente $\gamma$, o que, em última análise, se reduz à desigualdade

$$
\left|\alpha^{\gamma}-\beta^{\gamma}\right| \leq|\alpha-\beta|^{\gamma}
$$

a qual vale para quaisquer reais $\alpha, \beta \geq 0$. Para prová-la, podemos supor, sem perda de generalidade, que $\alpha>\beta>0$. Dividimos (1.24) por $\beta^{\gamma}$ e encontramos a expressão equivalente

$$
x^{\gamma}-1 \leq(x-1)^{\gamma}, \text { para } x \geq 1,
$$

que pode ser deduzida com recursos do Cálculo de uma variável real.

\subsection{Funções Analíticas}

\section{Diferenciabilidade}

Sejam E e F espaços normados sobre o corpo dos números complexos e consideremos uma aplicação $f$ de aberto $U \subset E$ em $F$. Diremos que $f$ é diferenciável no sentido de Fréchet em um ponto $\xi$ de $U-$ ou, simplesmente, diferenciável - quando tivermos

$$
\lim _{x \rightarrow \xi} \frac{\left\|f(x)-f(\xi)-A_{\xi}(x-\xi)\right\|_{F}}{\|x-\xi\|_{E}}=0,
$$

onde $A_{\xi}$ é uma transformação linear limitada de $E$ em $F$. Usualmente, denotamos $A \xi$ por $D f(\xi)$, o qual será referido doravante como sendo o diferencial de $f$ em $\xi$. A noção de diferenciabilidade é topológica, isto é, se $f$ for diferenciável com respeito à um determinado par de normas em $E$ e $F$, então $f$ o será com respeito a quaisquer outras normas equivalentes.

Propriedades elementares sobre o diferencial, como a regra da cadeia, linearidade da aplicação que associa cada função ao seu diferencial num ponto fixado, entre tantas outras, podem ser encontradas em [5], a partir da página 54.

Dados dois pontos $a$ e $b$ de $E$, definimos o segmento linear $[a, b]$ unindo estes dois pontos como sendo o conjunto de todas as combinações lineares $(1-\lambda) a+\lambda b, \operatorname{com} \lambda$ variando no intervalo unidade $[0,1]$. Este conjunto pode ser naturalmente identificado com qualquer intervalo real de tamanho $\|b-a\|$, herdando, assim, uma relação de ordem e a propriedade do supremo. 
Teorema 1.10 (Desigualdade do valor médio) $S e\|D f(x)\| \leq M$ sobre $[a, b] \subset U$, então

$$
\|f(b)-f(a)\|_{F} \leq M\|b-a\|_{E} .
$$

De agora em diante, abandonaremos os índices que indicam o espaço onde estão definidas as normas.

DemonstraçÃo. Seja $\Lambda_{\varepsilon}$ o conjunto dos pontos $x$ do segmento $[a, b]$ que verificam

$$
\|f(x)-f(a)\| \leq(M+\varepsilon)\|x-a\| .
$$

Este conjunto é fechado (por continuidade) e não vazio (pois contém $a$ ) e, portanto, contém o seu supremo $c$. Mostraremos que $c=b$, o que terminará a demonstração, pois $\varepsilon>0$ é arbitrário. Suponhamos, por absurdo, que $c$ seja estritamente menor que $b$. Usamos diretamente a diferenciabilidade de $f$ em $c$ para concluir que

$$
\|f(t)-f(c)\| \leq(M+\varepsilon)\|t-a\|
$$

para todo $t$ numa vizinhança $V \subset U$ de $c$. Escolhemos um ponto $t$ de $V$ sobre o segmento $[a, b]$ de modo que, na ordem de $a$ para $b$ em $[a, b]$, tenhamos $c<t<b$. Observemos então que $\|t-a\|$ é igual à soma de $\|t-c\|$ com $\|c-a\|$. Deste modo,

$$
\|f(t)-f(a)\| \leq\|f(t)-f(c)\|+\|f(c)-f(a)\| \leq(M+\varepsilon)\|t-a\|,
$$

o que nos mostra que $t$ está em $\Lambda_{\mathcal{E}}$, contradizendo o fato de $c$ ser o supremo de $\Lambda_{\mathcal{E}}$.

\section{Diferenciais de ordem superior}

Suponha que $D f(x)$ esteja definida numa vizinhança $V$ de $\xi \in U$. Se a aplicação de $D f$, definida em $V$ e tomando valores no espaço normado complexo $L(E, F)$ (conjunto dos operadores lineres limitados com a norma usual de operadores) for diferenciável no ponto $\xi$, então diremos que $f$ é duas vezes diferenciável no ponto $\xi$. O diferencial de $D f$ no ponto $\xi$ será denotado por $D^{2} f(\xi)$. De modo inteiramente análogo, podemos definir diferenciabilidade de ordem $m \geq 2$ num ponto $\xi$ de $U$, bem como diferenciais

$$
D^{m} f \in L(E, L(E, L(E, L(\cdots, L(E, F))))) \simeq L^{m}(E, F)
$$

que são naturalmente identificados com uma correspondente forma $m$-linear do espaço $L^{m}(E, F)$, denotada por $d^{m} f$. Para mais detalhes, ver [5], capítulo 7.

Diremos que uma forma $m$-linear $A$ de $L^{m}(E, F)$ é simétrica quando o valor de $A\left(x_{1}, \ldots, x_{m}\right)$ for independente da ordem em que os $x_{i}$ aparecem nas entradas.

Teorema 1.11 (Schwarz) Se $f$ for m-diferenciável em $\xi$, então $d^{m} f(\xi)$ será uma forma simétrica. (Referência: [5], página 87). 
Agora introduziremos um conceito equivalente ao de derivada direcional nos espaços euclidianos. Para um certo $x \in E$, podemos considerar o limite formal ${ }^{7}$

$$
\frac{\partial f}{\partial x}(\xi)=\lim _{\lambda \rightarrow 0} \frac{f(\xi+\lambda x)-f(\xi)}{\lambda}
$$

também chamado de derivada direcional de $f$ em $\xi$ na direção de $x$, ou derivada de Gâteaux de $f$ em $\xi$ na direção de $x$. Ressaltamos $\lambda$ é um escalar complexo. Se a aplicação $\partial f(\xi)$ que associa à cada $x$ em $E$ a derivada direcional $\partial f(\xi) / \partial x$ estiver bem definida, então diremos que $f$ é diferenciável no sentido de Gâteaux em $\xi$, ou que $f$ é Gâteaux diferenciável em $\xi$, e chamaremos $\partial f(\xi)$ de a derivada de Gâteaux de $f$ em $\xi$. É fácil ver, pela própria definição de diferenciabilidade, que

Proposição 1.10 Se f for diferenciável no sentido de Fréchet em $\xi$, então $f$ também o será no sentido de Gâteaux, com $D f(\xi)=\partial f(\xi)$.

Não é sempre verdade que a derivada de Gâteaux seja uma aplicação linear, tampouco que toda função Gâteaux diferenciável em $\xi$ seja contínua neste ponto. De fato, se considerarmos $E$ como sendo o plano euclidiano dos pares $x=\left(x_{1}, x_{2}\right)$ e definirmos

$$
f(x)=x_{1} x_{2}^{2} /\left(x_{1}^{2}+x_{2}^{2}\right), \quad f(0)=0,
$$

então veremos que a derivada direcional de $f$ na origem coincide com a própria função, i. é., $\partial f(0) / \partial x=f(x)$, o que mostra que a derivada de Gâteaux no ponto 0 não pode ser linear, neste caso. Já

$$
f(x)=x_{1}^{3} / x_{2}, f(0)=0
$$

nos oferece um exemplo de uma função Gâteaux diferenciável na origem que não é contínua neste ponto.

Uma vez definidas as derivadas direcionais, podemos enunciar o Teorema de Schwarz na sua forma clássica.

Teorema 1.12 (Schwarz) Suponha que $f$ seja duas vezes diferenciável sobre todo o aberto U. Então

$$
\frac{\partial^{2} f}{\partial x \partial y}(\xi)=d^{2} f(\xi)(x, y)=\frac{\partial^{2} f}{\partial y \partial x}(\xi)
$$

para todo $\xi$ em $U$ e quaisquer $x, y \in E$.

Referência: [5], página 91.

\subsubsection{Teorema da função inversa (e implícita) para espaços de Banach}

Todos os espaços normados relacionados à estes dois Teoremas, que apresentaremos logo mais, são completos, i.é., vamos considerar somente espaços de Banach (complexos ou reais). Diremos que $f$ é de classe $C^{m}$ sobre $U$, para um certo $m \geq 1$, se $D^{m} f$

\footnotetext{
${ }^{7}$ A próxima igualdade também define uma notação padrão para este limite.
} 
existir e for contínuo sobre $U$. Se $f$ for $C^{m}$ e, além disso, for uma aplicação bijetiva de $U$ sobre um aberto $V$ contido em $F$, cuja inversa também é $C^{m}$, então diremos que $f$ é um difeormofismo de classe $C^{m}$ de $U$ sobre $V$.

Agora vejamos o problema de se determinar a inversa de uma aplicação de classe $C^{m}$. Se $\xi$ for um ponto de $U$, então diremos que $f$ é um difeomorfismo local de classe $C^{m}$ em $\xi$ quando existirem vizinhanças $V(\xi)$ e $W(f \xi)$ tais que $f$ é um difeomorfismo ${ }^{8}$ de classe $C^{m}$ se $V$ sobre $W$. Afirmamos então que

Teorema 1.13 (da função inversa) Uma condição necessária para que $f$, uma aplicação de classe $C^{m}$ sobre $U$, seja um difeomorfismo local de classe $C^{m}$ em $\xi \in U$ é que seu diferencial $D f(\xi)$ seja um isomorfismo linear neste ponto.

Referência: [5], página 94.

Este Teorema é um dos resultados fundamentais sobre diferenciabilidade e possui aplicações muito importantes em diversos ramos da matemática, como Teoria Espectral e Sistemas Dinâmicos. Em nosso trabalho, por exemplo, usaremos o Teorema da função implícita - que, de fato, é um variante do Teorema da função inversa - para mostrar a analiticidade real da dimensão de Hausdorff do conjunto de Julia de uma função racional hiperbólica com respeito à perturbações do sistema.

Como acabamos de afirmar, o Teorema da função implícita é uma variante do Teorema da função inversa. De fato, tudo que precisamos é desenvolver uma notação apropriada para expressar este resultado. Por exemplo, se tivermos uma função

$$
g: E \times F \supset U \times V \rightarrow G
$$

então podemos considerar separadamente as funções $\varphi=g(x, \cdot)$ e e $\psi=g(\cdot, y)$, para $x$ e $y$ fixos. Se $g$ for diferenciável num ponto $(x, y)$ então é imediato que estas duas funções também o serão. Definimos, então,

$$
D_{1} g(x, y)=D \varphi(y), D_{2} g(x, y)=D \psi(x)
$$

como sendo as derivadas parciais de $g$ com respeito à primeira e segunda variáveis, respectivamente. Agora suponhamos que $G$ seja um produto $J \times K$ de outros dois espaços normados $J$ e $K$. Neste caso, pode decompor $g$ em termos de suas funções coordenadas, escrevendo $g=\left(g_{1}, g_{2}\right)$. Este processo nos permite expressar $D g$ em termos de $D_{j} g_{i}$ com o auxílio de uma forma matricial. De um modo geral, se $A_{11}, A_{12}, A_{21} \mathrm{e}$ $A_{22}$ forem transformações lineares de $L(E, J), L(F, J), L(E, K)$ e $L(F, K)$, respectivamente, então a forma matricial ${ }^{9}\left(A_{i j}\right)$ representa o operador linear $B \operatorname{de} L(E \times F, J \times K)$ agindo sobre o produto $E \times F$ como um simples produto de matriz por vetor, ou seja

$$
B\left(h_{1}, h_{2}\right)=\left(A_{11} h_{1}+A_{12} h_{2}, A_{21} h_{1}+A_{22} h_{2}\right) .
$$

\footnotetext{
${ }^{8} \mathrm{~A}$ notação $V(p)$ indica uma vizinhança do ponto $p$.

${ }^{9}$ Com esta notação, $i$ sempre é o índice da linha e $j$ o da coluna.
} 
No caso da aplicação diferenciável $g$, a forma matricial do operador diferencial $D g$ é $\left(D_{j} g_{i}\right)$, onde, naturalmente, omitimos o ponto onde o diferencial é calculado, mas isso é irrelevante.

Voltemos ao Teorema da função implícita. Consideremos três espaços de Banach, E, F e G. Seja $f$ uma aplicação de $U \times V$ em $G$, onde $U$ é um aberto de $E$ e $V$ um aberto de $F$. O problema que se nos apresenta é o de dizer quando a expressão

$$
f(x, y)=w_{0}
$$

define implicitamente uma função $y=y(x)$, pelo menos localmente. Queremos, também, determinar condições que garantam a regularidade desta função.

Teorema 1.14 Se f for de classe $C^{m}$ e se $D_{2} f(a, b)$ for um isomorfismo, então existirão vizinhanças $U_{0}(a)$ e $W_{0}(f(a, b))$, e uma única aplicação $C^{m}$-diferenciável $g$ de $U_{0} \times W_{0}$ em $V$ que satisfaz.

$$
f(x, g(x, w))=w
$$

para todo $(x, w)$ em $U \times W$.

DemonstraçÃo. Definimos $\phi(x, y)=(x, f(x, y))$ sobre $U \times V$, que claramente é diferenciável e de classe $C^{m}$; colocando-se $D \phi(a, b)$ na forma matricial fica fácil ver que, como $D_{2} f(a, b)$ é um isomorfismo, o mesmo acontece com o diferencial $D \phi(a, b)$. Pelo Teorema da função inversa, existe uma inversa local $\psi_{0}$ de classe $C^{m}$ e definida sobre uma vizinhança $U_{0} \times W_{0}$. Naturalmente, esta inversa deve ser da forma

$$
\psi_{0}(x, w)=(x, g(x, w))
$$

o que faz com que $g$ seja, necessariamente, de classe $C^{m}$ (note que $g$ é a composição de uma função $C^{m}$ com uma projeção, que é linear, e portanto, $C^{m}$; de modo que o resultado segue da regra da cadeia). Um simples cálculo nos mostra que $g$ verifica (1.25). Por outro lado, se $g_{1}$ fosse uma outra função satisfazendo às mesmas condições, então

$$
\psi_{1}(x, w)=\left(x, g_{1}(x, w)\right)
$$

seria uma inversa à direita de $\phi$ sobre $U_{0} \times W_{0}$, o que faz com que $\psi_{1}$ seja igual à $\psi_{0} \mathrm{e}$, portanto, $g=g_{1}$.

Corolário 1.1 (Teorema da função implícita) Suponhamos que $f$ seja de classe $C^{m}$ e que $D_{2} f(a, b)$ seja um isomorfismo. Seja $w_{0}=f(a, b)$. Então existem vizinhanças $U_{0}$ de a e $V_{0}$ de $b$ tais que,

$$
\left(\forall x \in U_{0}\right)\left(\exists ! y \in V_{0}\right)\left(f(x, y)=w_{0}\right) .
$$

Esta correspondência define uma aplicação $y=\ell(x)$ que é de classe $C^{m}$ sobre $U_{0}$. 
DEMONSTRAÇÃo. Seja $P_{2}$ a projeção canônica de $U \times V$ sobre $V$. Usando a mesma notação da demonstração do teorema, sabemos que a aplicação $\psi_{0}$ estabelece uma correspondência biunívoca entre pontos $(x, w)$ de $U_{0} \times W_{0}$ e pontos $(x, y)$ de uma vizinhança $N$ de $(a, b)$, com $w=f(x, y)$. Deste modo, para cada $x \in U_{0}$ existe um único $y$ em $P_{2} N$ tal que

$$
(x, f(x, y))=\left(x, w_{0}\right) .
$$

Esta correspondência define uma aplicação $y=\ell(x)$, como em (1.26). Mas a função $g\left(\cdot, w_{0}\right)$ satisfaz a mesma propriedade que determina $\ell$ e, portanto, $\ell=g\left(\cdot, w_{0}\right)$.

\subsubsection{Séries de Potências}

Consideremos dois espaços normados E e F sobre um mesmo corpo ( $\mathbf{R}$ ou $\mathbf{C})$.

Funções analíticas, em linhas gerais, são aquelas que podem ser localmente representadas como uma soma polinomial infinita. Tais somas, mais apropriadamente chamadas de séries de potências, serão o nosso objeto de estudo. É com o intuito de entender a natureza local de uma função analítica que apresentaremos as propriedades básicas destas séries.

Uma aplicação $P$ de $E$ em $F$ para a qual existe uma forma $m$-linear $A$ de $E^{m}$ em $F$, não necessariamente contínua, tal que

$$
P(x)=A(x, x, \ldots, x)=A x^{m}
$$

é chamado de polinômio homogêneo de grau $m$. O caso em que $m=0$ corresponde ao conjunto das funções constantes de $E$ em $F$, comumente identificado com o próprio $F$. Naturalmente, toda forma $m$-linear dá origem à um polinômio homogêneo. Fato curioso, porém, é que podemos tomar estas formas sempre como sendo simétricas, ou seja

Proposição 1.11 Se P for um polinômio homogêneo de grau $m \geq 1$, então existirá uma única forma simétrica e m-linear $A$, de $E$ em $F$, tal que $P(x)=A x^{m}$.

Referência: Ver [5].

Deste modo, temos uma correspondência biunívoca entre polinômicos homogêneos e formas simétricas. Se $P$ for proveniente de uma forma simétrica $A$, então escreveremos

$$
P=\widehat{A} .
$$

Todo polinômio homogêneo é uma função a valores vetoriais, e, com isso, podemos considerar combinações lineares finitas de polinômios homogêneos, dando origem aos chamados polinômios. Pode-se mostrar que

Proposição 1.12 Todo polinômio se escreve do modo único como soma finita de polinômios homogêneos de graus distintos. Além disso, $P=\widehat{A}$ será uma aplicação contínua precisamente quando a forma simétrica A for limitada, o que, em termos de $P$, é equivalente a dizer que existe uma constante $M>0$ satisfazendo 


$$
\|P(x)\| \leq M\|x\|^{m}
$$

(Referência: [5], página 41).

Denotaremos o ínfimo de todos os $M$ positivos que satisfazem a desigualdade acima por $\|P\|$, também chamado de norma do polinômio $P$. Recordemos que, de modo inteiramente análogo, uma forma $m$-linear $A$ será contínua precisamente quando existir uma constante positiva $M$ que verifique

$$
\left\|A\left(x_{1}, x_{2}, \ldots, x_{m}\right)\right\| \leq M\left\|x_{1}\right\| \times \cdots \times\left\|x_{m}\right\| .
$$

Em vista disso, empregamos o termo limitada para dizer que a forma $A$ é contínua. A menor das constantes $M$ acima, denotada $\|A\|$, é chamada de norma da forma $m$ linear $A$.

Proposição 1.13 As normas de um polinômio homogêneo contínuo e de sua correspondente forma simétrica estão relacionadas por

$$
\|\widehat{A}\| \leq\|A\| \leq \frac{m^{m}}{m !}\|\widehat{A}\| .
$$

Referência: [5].

Agora estudaremos somas infinitas de polinômios homogêneos; mais especificamente, se $\xi$ for um ponto de $E$ e $A_{m}$ uma sequência de formas simétricas contínuas de $E^{m}$ para $F$, então a soma formal ${ }^{10}$

$$
\sum_{m=0}^{\infty} A_{m}(x-\xi)^{m}=\sum_{m=0}^{\infty} \widehat{A}_{m}(x-\xi)
$$

será uma série de potências centrada em $\xi$. .

Podemos nos perguntar quando estas somas formais possuem algum significado como funções, ou seja, determinar os valores de $x$ que fazem com que a correspondente série em $F$ seja convergente. Nos dispensamos, aqui, de definir o que seja convergência uniforme e pontual; os conceitos são os mesmos que encontramos em Cálculo e Análise no espaço euclidiano.

Definimos o raio de convergência uniforme desta séria como sendo o supremo de todos os números não-negativos $r$ para os quais a série converge uniformemente sobre a bola fechada $\|x-\xi\| \leq r$. Diremos que a série converge absolutamente num ponto $x$ se tivermos

$$
\sum_{m=0}^{\infty}\left\|A_{m}(x-\xi)^{m}\right\|<\infty
$$

e definimos o raio de convergência absoluta como sendo o supremo dos números nãonegativos $r$ para os quais a série converge absolutamente em todo ponto de $\|x-\xi\| \leq r$.

\footnotetext{
${ }^{10}$ Convenção: $A_{0}(x-\xi){ }^{0}=A_{0}$. Podemos ver $A_{0}$ como uma constante $A_{0} \in F$.
} 
Podemos associar à série formal (1.27) os números $\rho$ e $\bar{\rho}$ dados por

$$
\frac{1}{\rho}=\limsup _{m \rightarrow \infty}\left\|\widehat{A}_{m}\right\|^{1 / m}, \quad \frac{1}{\bar{\rho}}=\limsup _{m \rightarrow \infty}\left\|A_{m}\right\|^{1 / m}
$$

Proposição 1.14 Se F for um espaço de Banach, então $\rho$ terá o mesmo valor que o raio de convergência uniforme de (1.27). Além disso, para todo r em $(0, \rho)$, temos

$$
\sum_{m=0}^{\infty}\left\|\widehat{A}_{m}\right\| r^{m}<\infty
$$

sendo que $\rho$ e $\bar{\rho}$ estão relacionados através da desigualdade $\rho / \mathrm{e} \leq \bar{\rho} \leq \rho$.

Referência: [5], páginas 153 e 156.

Em particular, segue do resultado acima que o raio de convergência absoluta é sempre maior ou igual que o raio de convergência uniforme. No caso de funções complexas e uma variável complexa, sabemos que estes raios coincidem. Mas em dimensão infinita existem exemplos em que essa desigualdade é estrita. De fato, consideremos o espaço de Banach $\ell^{2}$ das sequências cujo quadrado é somável (para uma descrição deste espaço, consultar qualquer livro introdutório de Análise Funcional). Deixamos a cargo do leitor verificar que a expressão

$$
P_{m}(x)=\left(x_{m}\right)^{m}
$$

define um polinômio homogêneo de grau $m$, onde $x=\left(x_{i}\right)$, e que a norma deste polinômio vale 1 , o que, pela Proposição anterior, faz com que o raio de convergência uniforme da série correspondente (soma dos $P_{m}$ ) seja 1. Para se calcular a norma de $P_{m}$, será conveniente mostrar que tal polinômio é proveniente da forma $m$-linear, de $\ell^{2}$ em $\mathbf{C}$, dada por

$$
A_{m}\left(x^{(1)}, \ldots, x^{(m)}\right)=x_{m}^{(1)} \times \cdots \times x_{m}^{(m)} .
$$

Por outro lado, em qualquer ponto $x$ de $\ell^{2}$, a série correspondente é comparável com a série geométrica de razão $1 / 2$ (a partir de um determinado índice), o que mostra que a série converge absolutamente em todo o espaço e, portanto, seu raio de convergência absoluta é infinito.

Teorema 1.15 (Unicidade de representação) Se duas séries de potências

$$
\sum_{m=0}^{\infty} P_{m}(x-\xi), \sum_{m=0}^{\infty} Q_{m}(x-\xi)
$$

convergirem absolutamente nalguma vizinhança de $\xi$ para uma mesma soma, então teremos $P_{m}=Q_{m}$, para todo $m \geq 0$. (Referência: [5], página 160). 
Agora consideremos o seguinte problema. Suponhamos que uma série como em (1.27) convirja em alguma bola $\|x-\xi\|<r$. A priori, não sabemos nada sobre o tipo de convergência. Nos estudos das propriedades locais de uma série de potências, muitas vezes será conveniente mudar o centro desta representação de $\xi$ para outro ponto $\eta$ da bola em questão. O próximo resultado nos diz como fazer isso, oferecendo ainda uma importante relação entre os coeficientes das duas séries.

Proposição 1.15 Suponhamos que F seja um espaço de Banach, e que o raio de convergência uniforme $R$ de (1.27) seja estritamente positivo. Denotemos por $f(x)$ a soma desta série na bola aberta $\|x-\xi\|<R$. Se $\eta$ for qualquer ponto desta bola, então poderemos escrever

$$
f(x)=\sum_{k=0}^{\infty} B_{k}(x-\eta)^{k}
$$

e o raio de convergência uniforme desta série será maior ou igual que a diferença $R-\|\eta-\xi\|$. Os coeficientes $B_{k}$ são dados por

$$
B_{k}=\sum_{m=k}^{\infty}\left(\begin{array}{l}
m \\
k
\end{array}\right) A_{m}(\eta-\xi)^{m-k}
$$

Referência: [5], página 161.

A última série consiste de uma soma de formas $k$-lineares e contínuas; mas ficou faltando dizer o que signicam os produtos de $A_{m}$ por $(\eta-\xi)^{m-k}$. Para $m=k$ este produto vale $A_{k}$ e, de um modo geral,

$$
A_{m}\left(x_{1}, \ldots, x_{m-k}\right)=A_{m}\left(x_{1}, \ldots, x_{m-k}, \cdot, \cdot, \ldots, \cdot\right),
$$

contando-se exatamente $k$ pontos “"” na expressão acima.

A esta altura, já temos todas as ferramentas em mãos para mostrar que toda série de potências que converge uniformemente é infinitamente diferenciável:

Teorema 1.16 Seja F um espaço de Banach. Se a série (1.27) possuir raio de convergência uniforme $R$ estritamente positivo, então sua soma $f(x)$ será uma função de classe $C^{\infty}$ sobre $\|x-\xi\|<R$, com

$$
A_{m}=\frac{1}{m !} d^{m} f(\xi) .
$$

DemonstraçÃo. Tomemos um $0<r<R$, arbitrário. Usando a Proposição 1.14, vemos que o supremo $d$ dos valores $\left\|\widehat{A_{m}}\right\| r^{m}$ é finito. Logo, para todo $x$ na bola $\| x-$ $\xi \|<r$, podemos escrever

$$
\left\|f(x)-f(\xi)-A_{1}(x-\xi)\right\|=\left\|\sum_{m=0}^{\infty} \widehat{A_{m}}(x-\xi)\right\| \leq d \sum_{m=0}^{\infty} \frac{\|x-\xi\|^{m}}{r^{m}}=\frac{d\|x-\xi\|^{2}}{r(r-\|x-\xi\|)}
$$


o que mostra que $f$ é diferenciável em $\xi$ e que seu diferencial neste ponto é $A_{1}$. Com o auxílio da última proposição, dado qualquer ponto $\eta$ de $\|x-\xi\|<R$, podemos escrever $f(x)$ como uma série de potências centrada em $\eta$ da forma (1.28). Aplicando-se o mesmo argumento que usamos no caso de $\xi$ para $x=\eta$, concluímos que $f(x)$ é diferenciável em $x=\eta$ e que o diferencial de $f$ neste ponto é $B_{1}$. Como $\eta$ é arbitrário, podemos escrever

$$
d f(x)=\sum_{m=0}^{\infty}(m+1) A_{m+1}(x-\xi)^{m}=\sum_{m=0}^{\infty} P_{m}^{(1)}(x-\xi)=\sum_{m=0}^{\infty} A_{m}^{(1)}(x-\xi)^{m},
$$

onde $P_{m}^{(1)} x=(m+1) A_{m+1} x^{m} \mathrm{e}$

$$
A_{m}^{(1)}:\left(x_{1}, \ldots, x_{m}\right) \mapsto(m+1) A_{m+1}\left(x_{1}, \ldots, x_{m}, \cdot\right) \in L(E, F) .
$$

O raio de convergência uniforme desta última série é maior ou igual que $R$, pois

$$
\left\|P_{m}^{(1)}\right\| \geq(m+1)\left\|P_{m+1}\right\|,
$$

o que pode facilmente ser verificado a partir da definição de norma de um polinômio. O mesmo procedimento que usamos até o momento aplica-se, portanto, à função $d f$ no lugar de $f$ (notemos que o contradomínio de $d f$ é um espaço de Banach), o que nos permite escrever

$$
d^{2} f(x)=\sum_{m=0}^{\infty}(m+1) A_{m+1}^{(1)}(x-\xi)^{m}=\sum_{m=0}^{\infty} A_{m}^{(2)}(x-\xi)^{m} .
$$

Novamente, o raio de convergência desta série é maior ou igual que $R$, pois

$$
\left\|P_{m}^{(2)}\right\|=\left\|\widehat{A_{m}^{(2)}}\right\| \geq(m+1)\left\|P_{m+1}^{(1)}\right\| \geq(m+1)(m+2)\left\|P_{m+2}\right\| .
$$

Podemos continuar este processo indefinidamente (indução), concluindo que

$$
d^{k} f(x)=\sum_{m=0}^{\infty}(m+1) \cdots(m+k) A_{m+k}(x-\xi)^{m} \text { e } d^{k} f(\xi)=k ! A_{k} .
$$

\subsubsection{Funções Analíticas}

Sejam E e F espaços normados sobre um mesmo corpo (real ou complexo), e consideremos uma função $f$, definida num aberto e conexo $U \subset E$, tomando valores em $F$.

Assumiremos que F é completo.

Diremos que $f$ é analítica se, em cada ponto $\xi$ de $U$, existir uma vizinhança $V(\xi)$ na qual $f$ se escreve como uma série de potências 


$$
f(x)=\sum_{m=0}^{\infty} A_{m}(x-\xi)^{m}
$$

centrada em $\xi$, com raio de convergência uniforme estritamente positivo. Tal série que quando existir, será única - também é conhecida como série de Taylor, ou desenvolvimento de Taylor de $f$ em torno do ponto $\xi$.

Toda função analítica deve ser, portanto, infinitamente diferenciável em seu domínio, e os coeficientes $A_{m}$ acima podem ser expressos em termos dos diferenciais de $f$ em $\xi$ através da fórmula (1.29). O caso que mais nos interessa é o complexo, ou seja, quando o corpo sobre o qual os espaços $E$ e $F$ estão definidos é $\mathbf{C}$. Neste caso, existe uma terminologia adicional: funções analíticas são também chamadas de holomorfas. O termo analítica real é sempre empregado quando queremos enfatizar que a diferenciabilidade é real e não complexa. Funções analíticas complexas (i.é., holomorfas) e reais gozam de propriedades distintas.

Toda função dada por um série de potências uniformemente convergente é analítica.

Proposição 1.16 Se duas funções analíticas $f$ e g definidas sobre $U$ coincidirem num aberto não vazio deste conjunto, então $f=g$ sobre $U$.

DEMOnSTRAÇÃo. A demonstração é muito simples e usa um argumento que se aplica em muitos casos. Coloquemos $h=f-g$ e seja $E$ o subconjunto de $U$ formado pelos pontos $x$ nos quais $f$ e todos os seus diferenciais se anulam. Todo ponto deste conjunto deve ser interior, visto que qualquer desenvolvimento de Taylor em torno destes pontos define uma função identicamente nula no aberto onde se dá a convergência uniforme para $f$. Por outro lado, seu complementar $E^{c}$ também é aberto, o que é consequência da continuidade de $f$ e de todas as suas derivadas: se $d^{m} f \neq 0$ em algum ponto $\xi$, então o mesmo acontece numa vizinhança $V$ de $\xi$, ou seja, $V \subset E^{c}$. Como $U$ é conexo, devemos ter $E=U$.

\subsubsection{Integração}

Nosso objetivo, agora, é desenvolver a fórmula integral de Cauchy para funções de espaços vetoriais normados (complexos) $E$ e $F$. Vamos supor que $F$ é completo. No que segue, $h(x)$ será uma função a valores em $F$, definida em algum aberto e conexo não-vazio $U \subset E$. A menos que se diga o contrário, $f(z)$ estará definida nalguma região $\Omega \subset \mathbf{C}$, tomando valores em $F$. Reservaremos $t$ para indicar qualquer ponto de um intervalo $[\alpha, \beta]$.

Primeiramente, definiremos integral de curvas vetoriais. Se $f(t)$ for contínua e a valores em $F$, então definimos

$$
\int_{\alpha}^{\beta} f(t) d t
$$

do mesmo modo como o fazemos no caso escalar, isto é, via somas de Riemann. A continuidade de $f(t)$ e o fato de $F$ ser completo são suficientes para garantir que estas somas convergem para um valor em $F$, o qual chamamos de integral de $f(t)$ sobre 
$[\alpha, \beta]$. Propriedades importantes da integral continuam válidas, tais como linearidade $\mathrm{e}$

$$
\left\|\int_{\alpha}^{\beta} f(t) d t\right\| \leq \int_{\alpha}^{\beta}\|f(t)\| d t .
$$

Seja $\gamma$ uma curva contida em $\Omega$, dada por $z=z(t)$. Diremos que $\gamma$ é diferenciável num ponto $t_{0}$ se

$$
z^{\prime}(t)=\lim _{s \rightarrow 0} \frac{z(t+s)-z(t)}{s}
$$

existir e for uma função contínua de $t$, para todo $t$ numa vizinhança de $t_{0}$ na topologia do intervalo $[\alpha, \beta]$ (logo, $t_{0}$ pode ser um ponto extremo). Notemos que, apesar do nome ser o mesmo, a terminologia "diferenciável" que usamos aqui para curvas não deve ser confundida com a que empregamos no caso geral de espaços normados. Se $z(t)$ for diferenciável em $[\alpha, \beta]$, exceto para um número finito de pontos $\left\{t_{i}\right\}_{1}^{n}$, então diremos que $\gamma$ é diferenciável por pedaços. Observemos que nos pontos $t_{i}, z(t)$ permanece sendo contínua. Assumiremos como condição adicional à definição de diferenciabilidade por pedaços que as derivadas laterais $z^{\prime}\left(t_{i}+\right)$ e $z^{\prime}\left(t_{i}-\right)$ existem, e que estas coincidem com os limites laterais de $z^{\prime}(t)$, à direita e à esquerda do ponto $t_{i}$, respectivamente.

Se $\gamma$ for diferenciável por pedaços e $f(z)$ contínua sobre $\gamma$, então definimos

$$
\int_{\gamma} f(z) d z=\int_{\alpha}^{\beta} f(z(t)) z^{\prime}(t) d t
$$

como sendo a integral de linha de $f$ ao longo da curva $\gamma$. Muitas propriedades elementares continuam válidas neste contexto mais geral. Por exemplo, se $f_{n}(z)$ forem contínuas e convergirem uniformemente para $f(z)$ sobre $\gamma$, então um argumento padrão nos mostra que $f(z)$ também será contínua sobre $\gamma$, e que vale

$$
\int_{\gamma} f(z) d z=\lim \int_{\gamma} f_{n}(z) d z
$$

Suponhamos, por um momento, que $f(z)$ seja diferenciável no seu domínio. Isso acarreta a existência do limite

$$
\lim _{h \rightarrow 0} \frac{f(z+h)-f(z)}{h}
$$

em cada ponto $z$ do conjunto $\Omega$. Denotaremos tal limite por $f^{\prime}(z)$, que é também chamado de derivada de $f$ no ponto $z$. Observemos que se $\lambda$ for um funcional linear contínuo de $F$, então a composição $\lambda \circ f(z)$ também será uma função diferenciável. No contexto de funções complexas de uma variável complexa, porém, sabemos que ser diferenciável é o mesmo que ser analítica. Logo, $\lambda \circ f$ é, de fato, uma função analítica. Usando a linearidade da integral e a definição de somas de Riemann, é fácil concluir que

$$
\lambda \int_{\gamma} f(z) d z=\int_{\gamma} \lambda \circ f(z) d z
$$


Portanto, se $f(z)$ for diferenciável num disco aberto $\Delta=\left\{\left|z-z_{0}\right|<r\right\}$, exceto por um número finito de pontos $^{11}\left\{\zeta_{j}\right\}_{1}^{n}$ nos quais

$$
\lim _{z \rightarrow \zeta_{j}}\left(z-\zeta_{j}\right) f(z)=0
$$

então

$$
w_{0}:=\int_{\gamma} f(z) d z=0
$$

para qualquer curva fechada e diferenciável por pedaços $\gamma$ contida em $\Delta$ que não passe por estes pontos. Este resultado, já conhecido do caso escalar, é consequência de (1.31) e do Teorema de Hahn Banach. Se $w_{0}$ fosse não nulo, então existiria um funcional linear limitado $\lambda$ de $F$ tal que $\lambda\left(w_{0}\right) \neq 0$, o que é impossível, já que a integral de $\lambda \circ f$ é sempre nula, qualquer que seja o funcional $\lambda$.

Uma consequência imediata deste resultado é a fórmula integral de Cauchy, que é obtida através da função

$$
Q(\zeta)=\frac{f(\zeta)-f(z)}{\zeta-z}
$$

onde $z$ é um número complexo arbitrário.

Assumiremos, agora, que $f(z)$ é diferenciável em todo o disco $\Delta$, no qual $\gamma$ está contida (podemos enfraquecer esta hipótese e admitir alguns pontos como exceção, como o fizemos anteriormente, mas não precisaremos de tanto). Observamos que $Q(\zeta)$ é diferenciável em $\Delta$, exceto (possivelmente) por $z$, onde $Q$ satisfaz a condição dada em (1.32), trocando-se $f(z)$ por $Q(\zeta)$ e $\zeta_{j}$ por $z$. Portanto, a integral de $Q$ ao longo de $\gamma$ é nula, o que nos permite escrever

$$
f(z) \int_{\gamma} \frac{1}{\zeta-z} d \zeta=\int_{\gamma} \frac{f(\zeta)}{\zeta-z} d \zeta
$$

Reconhecemos na primeira integral da igualdade acima o número $n(\gamma, z) 2 \pi i$, onde $n(\gamma, z)$ é o índice do ponto $z$ com respeito à curva $\gamma$. Este índice é sempre um número inteiro, e seu valor conta o número de voltas que a curva $\gamma$ dá em torno do ponto $z$ no sentido anti-horário. É claro que esta última noção carece de definição formal, mas o fato é que ela está implícita na própria integral que define o índice. Pode-se mostrar facilmente que o índice de qualquer ponto $z$ com respeito ao círculo $\left|z-z_{0}\right|=R$, parametrizado no sentido anti-horário, é 0 ou 1, dependendo da posição de $z$ relativa ao círculo, i.é., se $z$ estiver no exterior ou no interior. Para mais propriedades do índice, ver [1].

Com isso, temos a fórmula integral de Cauchy

\footnotetext{
${ }^{11}$ Observe que, nestes pontos, $f$ não precisa sequer estar definida.
} 


$$
f(z) n(\gamma, z)=\frac{1}{2 \pi i} \int_{\gamma} \frac{f(\zeta)}{\zeta-z} d \zeta
$$

Resumiremos o que foi deduzido no

Teorema 1.17 Se $f(z)$ for diferenciável num disco $\Delta=\left\{\left|z-z_{0}\right|<r\right\}$, e se $\gamma$ for uma curva fechada, diferenciável por pedaços e contida em $\Delta$, então (1.34) será válida para todo $z \in \mathbf{C}$ que não esteja sobre $\gamma$.

A importância da fórmula integral de Cauchy reside no fato de ser possível obter os valores de $f(z)$ dentro de um disco através de uma integral que leva em conta somente os valores de $f$ no bordo deste conjunto, tomando o ponto $z$ como um simples parâmetro. Naturalmente, a fórmula que exibimos em (1.34) nos fornece informação sobre $f(z)$ somente no caso em que $z$ estiver no disco, pois, de outro modo, o índice seria zero.

Nosso objetivo, agora, será extender a fórmula integral de Cauchy para $h(x)$. Para tanto, vamos supor que $h(x)$ é Fréchet diferenciável em $U$. Seja $\xi$ um ponto de $U$, e consideremos o conjunto $V$, formado pelos pontos $x \in U$ para os quais existe $R>1$ satisfazendo

$$
\Delta_{R}=\{|z|<R\} \subset\{z \in \mathbf{C}: \xi+z(x-\xi) \in U\} .
$$

Fixemos $x \in V$ e um correspondente valor de $R$. Então a função

$$
g(z)=h(\xi+z(x-\xi))
$$

é diferenciável no disco $\Delta_{R}$, o qual contém um círculo $|z|=r$, para algum $r$ no intervalo $(1, R)$. Podemos aplicar o Teorema anterior à esta função, obtendo

$$
g(1)=\frac{1}{2 \pi i} \int_{|z|=r} \frac{g(z)}{z-1} d z
$$

Teorema 1.18 (Fórmula integral de Cauchy) De acordo com as hipóteses acima,

$$
h(x)=\frac{1}{2 \pi i} \int_{|z|=r} \frac{h(\xi+z(x-\xi))}{z-1} d z
$$

para todo $x$ em $V$.

A expressão que obtemos para a fórmula acima não corresponde à forma escalar; mas uma pequena mudança de variáveis nos mostra que elas são as mesmas no caso em que $E=\mathbf{C}$. Contudo, mesmo que não fosse possível deduzir uma da outra, nem por isso a expressão que obtemos deixaria de ter o seu valor como "fórmula integral" (ver o primeiro parágrafo depois do Teorema 1.17).

Podemos, também, desenvolver uma fórmula integral para os diferenciais $d^{m} h(\xi)$. Para tanto, vamos assumir que $h$ é uma função holomorfa. Fixemos um $\xi$ em $U$ e tomemos um $x_{0} \in E$, arbitrário. Como $U$ é aberto, existe um valor positivo de $R$ tal que $\xi+z x_{0}$ está em $U$ sempre que $z \in \Delta_{R}$. Definimos 


$$
g(z)=\frac{h\left(\xi+z x_{0}\right)}{z^{m+1}}
$$

sobre $\Delta_{R}^{*}$, que é obtido de $\Delta_{R}=\{|z|<R\}$ pela exclusão do zero. Trata-se de uma função diferenciável no seu domínio, qualquer que seja $m \geq 0$. Seja $r$ um número real em $(0, R)$ e escolhamos um $s$ em $(0, r)$, pequeno o suficiente para que: (i) a série de Taylor

$$
h(x)=\sum_{k=0}^{\infty} P_{k}(x-\xi)
$$

de $h$ em torno do ponto $\xi$ convirja uniformemente sobre a bola fechada $\|x-\xi\| \leq s$ e (ii) $s /\left\|x_{0}\right\|$ esteja no intervalo $(0, r)$. Por um momento, vamos assumir que $x_{0}$ é não nulo. Uma aplicação conveniente de (1.33) nos mostra que

$$
\int_{|z|=s /\left\|x_{0}\right\|} g(z) d z=\int_{|z|=r} g(z) d z
$$

A vantagem desta igualdade é que a primeira integral pode ser calculada a partir da expansão de Taylor de $h(x)$, usando-se integração termo a termo. Este último recurso pode ser justificado neste contexto por um argumento muito simples; basta observar que toda soma de uma série é o limite de uma sequência de funções, e que a série convirgirá uniformemente precisamente quando a correspondente sequência de funções for uniformemente convergente. A integração termo a termo é obtida, então, a partir de (1.30) e do fato de $\int_{\gamma}$ ser um funcional linear sobre funções.

Escrevemos

$$
g(z)=\frac{1}{z^{m+1}} \sum_{k=0}^{\infty} P_{k}\left(z x_{0}\right)=\frac{P_{0}\left(x_{0}\right)}{z^{m+1}}+\cdots+\frac{P_{m}\left(x_{0}\right)}{z}+P_{m+1}\left(x_{0}\right)+P_{m+2}\left(x_{0}\right) z+\cdots,
$$

observando que a convergência desta série de potências é uniforme sobre o círculo $|z|=s /\left\|x_{0}\right\|$. Portanto $^{12}$,

$$
\int_{|z|=s /\left\|x_{0}\right\|} \frac{h\left(\xi+z\left(x_{0}-\xi\right)\right)}{z^{m+1}} d z=\int_{|z|=s /\left\|x_{0}\right\|} \frac{P_{m}\left(x_{0}\right)}{z} d z=2 \pi i P_{m}\left(x_{0}\right)=2 \pi i \frac{\widehat{d^{m} h(\xi)}\left(x_{0}\right)}{m !} .
$$

Uma comparação de (1.36) com (1.35) nos conduz a

$$
\widehat{d^{m} h(\xi)}\left(x_{0}\right)=\frac{m !}{2 \pi i} \int_{|z|=r} \frac{h\left(\xi+z x_{0}\right)}{z^{m+1}} d z
$$

que é a fórmula integral de Cauchy para diferenciais .

\footnotetext{
${ }^{12}$ Caso alguma passagem tenha ficado obscura nas próximas igualdades, sugerimos uma leitura das páginas 106 e 107 de [1].
} 
Teorema 1.19 Se $h$ for holomorfa e $\xi$ for um ponto qualquer de $U$, então, para todo $x_{0}$ em $E$ e todo $m \geq 0$, vale a fórmula (1.37), onde $r$ é qualquer número real positivo tal que

$$
\{|z| \leq r\} \subset\left\{z \in \mathbf{C}: \xi+z x_{0} \in U\right\} .
$$

Acabamos de demonstrar o caso $x_{0} \neq 0$. Por outro lado, se $x_{0}=0$, então a fórmula acima pode ser verificada diretamente: se $m \neq 0$, então ambos os lados na igualdade serão nulos e, caso contrário, iguais a $f(\xi)$.

\section{Holomorfia segundo Cauchy e Weierstrass}

Até o momento, o estudo que fizemos de holomorfia foi baseado no ponto de vista de Weierstrass: consideramos como holomorfa funções infinitamente diferenciáveis dadas localmente por uma série de potências. Contudo, uma condição muito mais fraca caracteriza estas funções. De fato, basta que uma aplicação entre espaços de Banach seja Fréchet Diferenciável para garantir que ela seja holomorfa. Poderíamos, deste modo, ter desenvolvido a teoria de funções holomorfas tomando como base o conceito de diferenciabilidade. Este é o ponto de vista de Cauchy. A seguir, faremos um breve resumo dos resultados principais que relacionam diferentes tipos de holomorfia, unificando todos os conceitos em apenas um.

Durante todo este tópico, E e F serão espaços de Banach complexos.

Historicamente, funções holomorfas eram aquelas aplicações

$$
w=f\left(z_{1}, \ldots, z_{n}\right)
$$

definidas num subconjunto aberto $U$ de $\mathbf{C}^{n}$ que fossem limitadas sobre compactos e holomorfas em cada variável $z_{j}$, separadamente. Esta última noção (ser holomorfa em cada variável) pode ser posta na seguinte forma. Seja $\left(a_{i}\right)_{1}^{n}$ um ponto de $U$ e fixemos uma posição $j$. Sabemos, então, que para valores $z$ próximos de $a_{j}$, os vetores $\varphi_{j}(z)=\left(z_{i}\right)$, onde $z_{i}=a_{i}$ para $i \neq j$ e $z_{j}=z$, ainda pertencem ao domínio de $f$; de modo que a função $f \circ \varphi_{j}(z)$ está localmente bem definida. Dizer que $f$ é holomorfa em cada variável separadamente é o mesmo que assumir que todas as funções $f \circ \varphi_{j}$ assim obtidas são holomorfas. A partir desta definição, consegue-se provar que $f$ é contínua; e do Lema de Osgood (Teorema 1.20) segue-se que $f$ é, de fato, holomorfa segundo a definição que temos usado até o momento. Existe, porém, um resultado mais forte na Teoria de funções analíticas, o qual nos diz que toda função holomorfa em cada variável deve ser contínua em todo o domínio $U$ e, portanto, holomorfa neste conjunto. Este fato é conhecido como Teorema de Hartogs; sua demonstração ${ }^{13}$ é bem mais difícil que a do Lema de Osgood.

Como estamos estudando funções definidas em $\mathbf{C}^{n}$, precisamos adaptar a notação de séries de potências para uma forma mais direta. Reservaremos as letras $z=\left(z_{i}\right)$ e

\footnotetext{
${ }^{13} \mathrm{O}$ enunciado que apresentamos do Teorema de Hartogs é preciso. Não simplificamos nenhuma hipótese. Para uma demonstração do Teorema de Hartogs, ver [26] pág. 28; ou então [29].
} 
$w=\left(w_{i}\right)$ para indicar elementos de $\mathbf{C}^{n}$. Se $r=\left(r_{i}\right)$ for um vetor de $n$ entradas positivas, então definimos os polidiscos

$$
\Delta(w, r)=\left\{z:\left|z_{j}-w_{j}\right|<r_{j} \forall j\right\} \text { e } \bar{\Delta}(w, r)=\left\{z:\left|z_{j}-w_{j}\right| \leq r_{j} \forall j\right\} .
$$

Recordemos que se $k$ for um número inteiro positivo, então $z^{k}=(z, \ldots, z)$ é um vetor de $k$ entradas, todas em $\mathbf{C}^{n}$. Podemos também definir potências para multíndices. Um multíndice $v$, de tamanho $n$, é uma $n$-upla de números inteiros não-negativos $v_{i}$. Definimos a norma $|v|$ do multíndice como sendo a soma de suas entradas. Também colocamos $z^{v}=z_{1}^{v_{1}} \cdots z_{n}^{v_{n}}$.

Uma vez definidos multíndices, podemos considerar somas formais do tipo

$$
\sum_{v} a_{v}\left(z-w^{0}\right)^{v}
$$

onde $a_{v}$ são números complexos e $w^{0}$ um elemento de $\mathbf{C}^{n}$. Tais somas são as mesmas séries de potências que já definimos anteriormente. Basta observar que os multíndices variam num conjunto enumerável e que a forma $|v|$-linear

$$
B_{v}\left(z^{(1)}, \ldots, z^{(|v|)}\right)=a_{v} z_{1}^{(1)} \cdots z_{1}^{\left(v_{1}\right)} z_{2}^{\left(v_{1}+1\right)} \cdots z_{2}^{\left(v_{1}+v_{2}\right)} \cdots z_{n}^{\left(v_{1}+\cdots+v_{n-1}+1\right)} \cdots z_{n}^{\left(v_{1}+\cdots+v_{n}\right)}
$$

define um polinômio homogêneo $P_{v}(z)=B_{v} z^{|v|}$ tal que

$$
a_{v}\left(z-w^{0}\right)^{v}=P_{v}\left(z-w^{0}\right)
$$

Como não existe nenhuma enumeração privilegiada dos multíndices, o tipo de convergência pontual em (1.38) deve ser sempre absoluta. Reciprocamente, deixamos a cargo do leitor o trabalho de tentar verificar que toda série de potências em $\mathbf{C}^{n}$ (com formas simétricas) pode convenientemente ser colocada na forma (1.38).

Teorema 1.20 (Lema de Osgood) Se uma função contínua $f$, definida num subconjunto aberto e não vazio $U$ de $\mathbf{C}^{n}$ e tomando valores em $\mathbf{C}$, for holomorfa em cada variável separadamente, então f será holomorfa.

DEMOnSTRAÇÃo. A demonstração se baseia no uso repetido da fórmula integral de Cauchy para funções de uma variável complexa.

Seja $w^{0}$ um ponto de $U$ e $r$ tal que o polidisco fechado $\bar{\Delta}\left(w^{0}, r\right)$ esteja contido em $U$. Vamos construir uma série de potências como (1.38), convergindo uniformemente em todo polidisco aberto $\Delta\left(w^{0}, r^{\prime}\right)$ de raio estritamente menor que $r$ (ou seja, $r_{j}^{\prime}<r_{j}$ ). Como $f$ é holomorfa em cada variável separadamente, podemos aplicar a fórmula integral de Cauchy $n$ vezes, obtendo 


$$
\begin{aligned}
& f\left(z_{1}, \ldots, z_{n}\right)=\frac{1}{2 \pi i} \int_{\left|\zeta_{1}-w_{1}^{0}\right|=r_{1}} \frac{f\left(\zeta_{1}, z_{2}, \ldots, z_{n}\right) d \zeta_{1}}{\zeta_{1}-z_{1}} \\
&=\left(\frac{1}{2 \pi i}\right)^{2} \int_{\left|\zeta_{1}-w_{1}^{0}\right|=r_{1}} d \zeta_{1} \int_{\left|\zeta_{2}-w_{2}^{0}\right|=r_{2}} \frac{f\left(\zeta_{1}, \zeta_{2}, z_{3}, \ldots, z_{n}\right) d \zeta_{2}}{\left(\zeta_{2}-z_{2}\right)\left(\zeta_{1}-z_{1}\right)} \\
&= \\
& \vdots \\
&=\left(\frac{1}{2 \pi i}\right)^{n} \int_{\left|\zeta_{1}-w_{1}^{0}\right|=r_{1}} d \zeta_{1} \int_{\left|\zeta_{2}-w_{2}^{0}\right|=r_{2}} d \zeta_{2} \int \ldots \\
& \cdots d \zeta_{n-1} \int_{\left|\zeta_{n}-w_{n}^{0}\right|=r_{n}} \frac{f(\zeta) d \zeta_{n}}{\left(\zeta_{1}-z_{1}\right) \ldots\left(\zeta_{n}-z_{n}\right)},
\end{aligned}
$$

para todo $z$ em $\Delta\left(w^{0}, r^{\prime}\right)$. O Teorema de Fubini nos permite escrever esta integral iterada como uma única integral sobre $\Upsilon=\left\{\zeta:\left|\zeta_{j}-w_{j}^{0}\right|=r_{j}\right\}$

$$
f(z)=\left(\frac{1}{2 \pi i}\right)^{n} \int_{\Upsilon} \frac{f(\zeta) d p(\zeta)}{(\zeta-z)^{i}}
$$

onde $d p$ é o produto $d \zeta_{1} \cdots d \zeta_{n}$ e $\imath$ é o multíndice de tamanho $n$ cujas entradas são todas iguais a 1 .

Observação. A notação que usamos em (1.40) é simplificada. De fato, $d p$ remete à uma integral sobre o cubo $[\alpha, \beta]^{n}$, onde $[\alpha, \beta]$ é o intervalo sobre o qual as curvas $\left|\zeta_{j}-w_{j}^{0}\right|=r_{j}$ estão parametrizadas. A situação é a mesma de uma única variável, em que uma integral de linha é, na verdade, uma integral sobre um intervalo, enquanto que o valor de $d z=z^{\prime}(t) d t$ é puramente formal. O conjunto $\Upsilon$, que aparece como domínio de integração, deve ser entendido como um conjunto de $n$ curvas parametrizadas no sentido anti-horário.

Usando um argumento iterativo envolvendo a soma da série geométrica de razão $\left(z_{j}-w_{j}^{0}\right) /\left(\zeta_{j}-w_{j}^{0}\right)$, conseguimos provar que

$$
\frac{1}{(\zeta-z)^{\imath}}=\sum_{v} \frac{\left(z-w^{0}\right)^{v}}{\left(\zeta-w^{0}\right)^{v+\imath}}
$$

converge uniformemente em $\zeta$, para cada $z$ fixado. A convergência também é absoluta em cada ponto. Logo, podemos integrar termo a termo, obtendo

$$
f(z)=\left(\frac{1}{2 \pi i}\right)^{n} \int_{\Upsilon} \sum_{v} \frac{f(\zeta)\left(z-w^{0}\right)^{v}}{\left(\zeta-w^{0}\right)^{v+\imath}} d p(\zeta)=\sum_{v}\left(\frac{1}{2 \pi i}\right)^{n} \int_{\Upsilon} \frac{f(\zeta) d p(\zeta)}{\left(\zeta-w^{0}\right)^{v+l}} \times\left(z-w^{0}\right)^{v} .
$$

A convergência da última série é absoluta e uniforme em $z$, mostrando que, com efeito, $f$ é analítica. 
A demonstração do Lema de Osgood possui consequências bem interessantes. Uma delas diz repeito ao raio de convergência uniforme de uma série de potências. Consideremos, por exemplo, uma função analítica $f(z)$, definida numa região $\Omega \subset \mathbf{C}^{n} \mathrm{e}$ tomando valores em $\mathbf{C}$. Sabemos que $f(z)$ pode localmente ser escrita como uma série de potências uniformemente convergente. Uma questão natural é saber até onde esta série converge, ou seja, qual o seu raio de convergência uniforme. Para simplificar o problema, suponhamos que $\Omega$ seja o polidisco unitário $\Delta^{n}(1)$ [ver índice de notação] e consideremos a representação local de $f(z)$ em torno da origem

$$
f(z)=\sum_{v} a_{v} z^{v}
$$

Seguindo a mesma notação empregada na demonstração do Lema de Osgood, tomemos uma curva $\Upsilon_{R}=\left\{\left|z_{i}\right|=R\right\}$. Para um dado multíndice $\mu$, a integral

$$
\int_{\Upsilon_{R}} \frac{z^{v}}{z^{\mu+\imath}} d p(z)
$$

será nula precisamente nos casos em que existir $i$ com

$$
v_{i}-\mu_{i}-1 \leq-2 \text { ou } v_{i}-\mu_{i}-1 \geq 0
$$

nos demais casos, deveremos ter $v=\mu$ e o valor da integral acima será $(2 \pi i)^{-n}$. Ao supor que a série converge (1.42) uniformemente numa vizinhança $V$ da origem, podemos tomar $\Upsilon_{R}$ contido em $V$; e da análise que fizemos sobre a integral (1.43) concluímos que

$$
a_{v}=\frac{1}{(2 \pi i)^{n}} \int_{\Upsilon_{R}} \frac{f(z)}{z^{v+\imath}} d p(z)
$$

Para obter (1.44), usamos que o sinal da integral "comuta" com o da soma da série no caso de convergência uniforme. ( $l$ é o multíndice unitário, como na demonstração do Lema de Osgood).

O que é de se notar, todavia, é que a integral em (1.44) permanece a mesma, independente do raio $R$ que tomemos, desde que $\Upsilon_{R} \subset \Delta^{n}(1)$. Assim, se $0<r<R<1$, então

$$
\left|a_{v}\right| \leq M\left(\frac{1}{R}\right)^{|v|},
$$

(onde $M$ é o supremo de $|f(z)|$ sobre $\overline{\Delta^{n}}(R)$ ) e a série (1.42) converge uniformemente sobre $\Delta^{n}(r)$. Consequentemente, o raio de convergência uniforme de (1.42) de ser 1 . Sobre essa última afirmação, convém lembrar que o raio de convergência (pelo menos à primeira vista) depende da norma do espaço. No caso de $\mathbf{C}^{n}$, esta será a norma do máximo, que estabelece uma correspondência natural entre bolas e polidiscos.

A soma da série (1.42) é uma função analítica em todo o polidisco unitário e coincide com $f(z)$ numa vizinhança da origem. Da Proposição 1.16 segue que a representação (1.42) é válida por todo o polidisco $\Delta^{n}(1)$.

No caso de uma região qualquer $\Omega$, o argumento acima nos permite dizer que 
Proposição 1.17 Seja $f(z)$ uma função holomorfa definida numa região $\Omega$ de $\mathbf{C}^{n}$, tomando valores em C. Dado $w^{0} \in \Omega$ e $R>0$ tal que $\Delta^{n}\left(w^{0}, R\right) \subset \Omega$, definimos

$$
a_{v}=\frac{1}{(2 \pi i)^{n}} \int_{\left|\zeta_{i}-w_{i}^{0}\right|=R} \frac{f(\zeta)}{\left(\zeta-w^{0}\right)^{v+l}} d p(\zeta) .
$$

Então a série

$$
\sum_{v} a_{v}\left(z-w^{0}\right)^{v}
$$

converge uniformemente sobre todo polidisco $\Delta^{n}\left(w^{0}, r\right)$ de raio $r<R$, e sua soma será $f(z)$ sobre este conjunto. Em particular, o raio de convergência uniforme da série (1.46) é o supremo dos $R>0$ tais que $\Delta^{n}\left(w^{0}, R\right) \subset \Omega$.

O Lema de Osgood é para funções à valores em C. Ao passarmos para o caso vetorial, observamos que uma função $f$ à valores em $\mathbf{C}^{m}$ será holomorfa se, e somente se, cada função coordenada $f_{i}$ o for. Além disso, como as projeções $\lambda_{i}(z)=z_{i}$ constituem uma base do dual de $\mathbf{C}^{m}$, a condição de cada função coordenada ser holomorfa é equivalente a dizer que $\lambda \circ f$ é holomorfa, para todo funcional linear de $\mathbf{C}^{m}$ (recordemos que todo funcional linear de um espaço de dimensão finita é limitado). Podemos generalizar este argumento, como mostra o seguinte resultado. Nele $-e$ nos demais dois resultados deste tópico -, E e F serão espaços de Banach complexos e $U$ um aberto de E.

Teorema 1.21 Uma função $f$ de $U$ em $F$ será holomorfa se, e somente se, $\lambda \circ f$ for holomorfa, para todo funcional linear limitado $\lambda$ de $F$.

Referência: [5], página 203.

Seja f uma aplicação de $U$ em $F$. Motivados pelo Teorema de Hartogs, diremos que $f$ é holomorfa no sentido de Gâteaux, ou G-holomorfa se, para todo $x$ em $E$ e todo $a \in U$, a função

$$
z \mapsto f(a+z x)
$$

for holomorfa. Diremos ainda que $f$ é localmente limitada se todo ponto $a$ de $U$ possuir uma vizinhança na qual $f$ é limitada.

Teorema 1.22 (Graves-Taylor-Hille-Zorn) As seguintes afirmações são equivalentes:

(1) f é holomorfa sobre $U$;

(2) $f$ é G-holomorfa e contínua sobre $U$;

(3) $f$ é G-holomorfa e localmente limitada sobre $U$.

Referência: [5], página 198.

Teorema 1.23 A função $f$ será Fréchet-diferenciável sobre $U$ se, e somente se, $f$ for holomorfa. 
DEMONSTRAÇÃO. Consequência dos resultados anteriores.

Exemplo - Aplicações holomorfas do espaço $C^{\gamma}(X)$ - Seja $X$ um espaço métrico compacto. Agora vamos explorar uma fonte abundante de exemplos de funções holomorfas do espaço de Banach $C^{\gamma}(X)$ nele mesmo. Começamos com uma função analítica complexa ${ }^{14} f(z)$ definida numa região $\Omega$ plano. Para cada $\phi \in C^{\gamma}(X)$ observamos que a aplicação

$$
f(\phi)(x):=f(\phi x)
$$

também está em $C^{\gamma}(X)$. Naturalmente, devemos exigir que a imagem de $\phi$ esteja contida num subconjunto compacto e convexo (para poder aplicar o Teorema do valor médio) de $\Omega$. Fixemos uma função $\phi_{0}$ de $C^{\gamma}(X)$ com essa propriedade. Então para toda $\phi \in C^{\gamma}(X)$ uniformemente próxima de $\phi_{0}, f(\phi)$ também está bem definida. Logo, a função $\phi \mapsto f(\phi)$, que também denotaremos por $f$ ou $f(\phi)$, está definida numa vizinhança de $\phi_{0}$, na topologia da norma $\|\cdot\|_{\gamma}$ de $C^{\gamma}(X)$. Recordemos que esta norma é dada por

$$
\|\phi\|_{\gamma}=\|\phi\|_{\infty}+\mathrm{C}_{\mathrm{H}}(\phi)
$$

onde $\|\phi\|_{\infty}$ é o supremo de $|\phi|$ sobre $X$ e $\mathrm{C}_{\mathrm{H}}(\phi)$ é a menor constante de Hölder de $\phi$.

Proposição 1.18 De acordo com as condições acima, $f(\phi)$ é Fréchet-diferenciável no ponto $\phi=\phi_{0}$, e sua derivada $D f\left(\phi_{0}\right)$ neste ponto é o operador de multiplicação por $f^{\prime}\left(\phi_{0}\right)$, ou seja,

$$
\left(D f\left(\phi_{0}\right) g\right)(x)=f^{\prime}(\phi x) g(x) .
$$

DEMONSTRAÇÃo. Seja $g(z)$ uma função analítica qualquer definida em $\Omega$ e consideremos um compacto $K_{0}$ contido em $\Omega$. Então existe um outro compacto $K_{1}$ contido em $\Omega$, cujo interior contém $K_{0}$, com a propriedade de que, para $\delta$ suficientemente pequeno, todo $h \in \mathbf{C}$ com $|h| \leq \delta$ satisfaz $h+K_{0} \subset K_{1}$. Podemos tomar, por exemplo, $K_{1}$ como sendo o conjunto dos pontos $z$ do plano com $d\left(z, K_{0}\right) \leq \varepsilon$, para um $\varepsilon$ suficientemente pequeno. Com isso, para $z \in K_{0}$ e $|h| \leq \delta$, a função

$$
R g(z, h)=g(z+h)-g(z)-g^{\prime}(z) h
$$

está bem definida. Para $h_{1}$ e $h_{2}$ no disco fechado de raio $\delta$, colocamos

$$
\Lambda\left(h_{1}, h_{2}\right)=\left\{h \in \mathbf{C}:|h| \leq \delta,|h| \leq\left|h_{1}\right|+\left|h_{2}\right|\right\}
$$

e observamos que, pelo Teorema 1.10 ,

$$
\begin{aligned}
\left|R g\left(z, h_{1}\right)-\operatorname{Rg}\left(z, h_{2}\right)\right| & \leq\left(\max _{h \in \Lambda\left(h_{1}, h_{2}\right)}\left|\frac{\partial \operatorname{Rg}(z, h)}{\partial h}\right|\right) \times\left|h_{1}-h_{2}\right| \\
& \leq \max _{h \in \Lambda\left(h_{1}, h_{2}\right)}\left(\max _{\zeta \in K_{1}}\left|g^{\prime \prime}(\zeta)\right|\right)|h| \times\left|h_{1}-h_{2}\right| \\
& \leq C_{g}\left(\left|h_{1}\right|+\left|h_{2}\right|\right)\left|h_{1}-h_{2}\right|
\end{aligned}
$$

${ }^{14} f(z) \neq \infty$, para todo $z$. 
onde $C_{g}$ é o máximo de $\left|g^{\prime \prime}(\zeta)\right|$ sobre $K_{1}$. Notemos que $C_{g}$ depende somente de $g$ e $K_{1}$. Com o auxílio da desigualdade que obtemos em (1.47) concluímos que

$$
|R f(z, h)-R f(w, h)| \leq C_{f^{\prime}}|h|^{2}|z-w|,
$$

para $z, w \in K_{0}$ e $|h| \leq \delta$.

Agora fixemos uma função $\phi$ em $C^{\gamma}(X)$, e seja $K_{0}$ um subconjunto compacto e convexo de $\Omega$ que contém a imagem de $\phi$. Seja $\delta$ o número encontrado acima e tomemos $h \in C^{\gamma}(X)$ de modo que $\|h\|_{\gamma} \leq \delta$. Em particular, temos $|h(x)| \leq \delta$, para todo $x$ em $X$. Seja

$$
R f(\phi, h)=f(\phi+h)-f(\phi)-f^{\prime}(\phi) h .
$$

Queremos mostrar que

$$
\frac{\|R f(\phi, h)\|_{\gamma}}{\|h\|_{\gamma}} \longrightarrow 0
$$

quando $\|h\|_{\gamma} \rightarrow 0$. Para tanto, usamos as desigualdades (1.47) e (1.48), obtendo

$$
\mathrm{C}_{\mathrm{H}}(R f(\phi, h)) \leq 2 C_{f} \mathrm{C}_{\mathrm{H}}(h)\|h\|_{\infty}+C_{f^{\prime}} \mathrm{C}_{\mathrm{H}}(\phi)\|h\|_{\infty}^{2}
$$

$\mathrm{e}$

$$
\|R f(\phi, h)\|_{\infty} \leq C_{f}\|h\|_{\infty}^{2}
$$

de onde segue que o quociente (1.49) tende à zero quando $\|h\|_{\gamma} \rightarrow 0$.

\subsubsection{Aplicações Conformes}

Entende-se por região do plano complexo qualquer subconjunto aberto, não-vazio e conexo. Assumiremos que $f(z)$ é uma função complexa, definida numa região $\Omega$. A definição de conformalidade que adotaremos é a seguinte:

Definição. Diremos que $f(z)$ é conforme quando esta for analítica e possuir derivada não-nula em qualquer ponto de seu domínio $\Omega$.

Esta definição, apesar de comum na maioria das referências, não é universal. Por isso, convidamos o leitor a abandoná-la, por um momento, para analisarmos cuidadosamente os conceitos que a motivaram, bem como suas consequências, que muitos autores acabaram adotando como sinônimo de aplicação conforme.

De todos os fatos associados à uma aplicação conforme, pelo menos um está sempre presente: toda aplicação conforme deve preservar ângulos, ou seja: (i) $f$ transforma curvas $\gamma \subset \Omega$ que possuem derivada não nula $\gamma^{\prime}\left(t_{0}\right)$ em curvas $\zeta=f \circ \gamma$ que também possuem derivada não nula no ponto $t_{0}$; e (ii) o ângulo entre quaisquer duas tangentes $\gamma_{1}^{\prime}\left(t_{0}\right)$ e $\gamma_{2}^{\prime}\left(t_{0}\right)$ não nulas é o mesmo que o ângulo ${ }^{15}$ formado pelos dois vetores

\footnotetext{
${ }^{15}$ A definição precisa de ângulo entre dois vetores não nulos $w$ e $z$, nesta ordem, é o argumento (i.é., um número real módulo $2 \pi$ ) do quociente $w / z$.
} 


$$
\left(f \circ \gamma_{1}\right)^{\prime}\left(t_{0}\right) \text { e }\left(f \circ \gamma_{2}\right)^{\prime}\left(t_{0}\right)
$$

Usando as curvas coordenadas $x(t)=(t, 0)$ e $y(t)=(0, t)$, vemos que toda aplicação conforme possui derivadas parciais não nulas; e se supormos que estas derivadas são contínuas (o que na prática sempre faremos), concluímos que $f(z)$ é analítica sobre $\Omega$ (ver [1], pág. 74).

A conformalidade ainda acarreta $f^{\prime}(z) \neq 0$ sobre $\Omega$, já que $f^{\prime}(z)=\partial f / \partial x$.

Reciprocamente, se $f(z)$ for analítica e com derivada não nula em todo ponto de $\Omega$, então é fácil ver que $f(z)$ preserva ângulos. Deste modo, a definição de conformalidade depende somente do tipo de regularidade que exigimos da função $f$. Como em todos os casos $f$ será analítica e, em particular, suas derivadas parciais de primeira ordem serão contínuas, justifica-se, deste modo, a definição que adotamos.

Podemos, de imediato, explorar algumas propriedades das aplicações comformes. De fato, segue do Teorema da função inversa que qualquer aplicação conforme $f$ é uma função aberta; e deste modo, se $f(z)$ for uma bijeção entre duas regiões $U$ e $V$, então $f$ será um homeomorfismo, com inversa igualmente conforme, pela regra de diferenciação da inversa.

Outro conceito associado às aplicações conformes é o de univalência. Uma função $f(z)$ será chamada de univalente quando esta for analítica e injetora no seu domínio.

Um dos fatos principais sobre aplicações univalentes é o Teorema da aplicação de Riemann (logo adiante); constitui fato elementar (mas não menos importante) que

Proposição 1.19 Se $f(z)$ for uma função univalente que leva uma região $U$ do plano bijetivamente noutra região $V$, então $f$ e sua inversa serão conformes.

Temos, assim, um meio alternativo de se definir conformalidade usando-se o conceito de univalência. Uma aplicação conforme e bijetiva entre duas regiões, cuja inversa também conforme, é chamada de equivalência conforme, ou de aplicação biconforme. Também empregaremos esta terminologia para quaisquer abertos não-vazios e conexos (regiões) de $\mathbf{C}^{N}$.

Dadas duas regiões simplesmente conexas do plano, podemos nos perguntar se elas são imagens uma da outra por meio de equivalências conformes. A resposta (afirmativa) para este fato consta na dissertação de Riemann (1851). A demonstração de Riemann sofreu algumas críticas nas décadas posteriores e, com isso, muitos matemáticos procuraram por uma prova mais rigorosa, dentre eles H. A. Schwarz (1943-1921), A. Harnack (1851-1888) e H. Poincaré (1854-1912), até que o objetivo foi alcançado em 1900, pelo matemático norte americano F. Osgood.

Teorema 1.24 (da Aplicação de Riemann) Dada qualquer região simplesmente conexa $S \subset \mathbf{C}$ que não seja o plano complexo todo, existe uma aplicação conforme e bijetiva $f$ de $S$ em $\{|w|<1\}$. Fixado qualquer ponto $z_{0}$ de $S$, esta aplicação será única sempre que especificarmos os valores $f\left(z_{0}\right) e \arg f^{\prime}\left(z_{0}\right)$.

A aplicação descrita no Teorema acima é univalente e, como tal, é uma equivalência conforme. A existência de tais aplicações, deste modo, vale para quaisquer regiões sim- 
plesmente conexas que não sejam o plano todo, bastando que se tome o disco unitário como região intermediária.

O leitor interessado numa demonstração pode consultar [1], pág. 222; e para maiores informações sobre a história do assunto, [48].

\subsection{Análise Complexa na esfera de Riemann}

Denotaremos por $\widehat{\mathbf{C}}$ a compactificação de um ponto do plano $\mathbf{C}$, comumente chamada de plano estendido. Este é um espaço topológico compacto, obtido de $\mathbf{C}$ pela adjunção de um único ponto extra, que denotaremos por $\infty$. A topologia deste espaço induzida sobre $\mathbf{C}$ coincide com a topologia original do plano, e a coleção de todos os complementares $\widehat{\mathbf{C}} \backslash K$ de compactos $K \subset \mathbf{C}$ é, por definição, uma base de vizinhanças de $\infty$. Muitas vezes, chamaremos $\infty$ de infinito, ou ponto no infinito. O conjunto $\widehat{\mathbf{C}}$ também herda as operações $+\mathrm{e} \cdot$ advindas do plano. Um pouco de prática com limites em $\mathbf{C}$ nos leva a definir

$$
\begin{aligned}
& a \cdot \infty=\infty \quad(a \in \widehat{\mathbf{C}}, a \neq 0) \\
& a / \infty=0 \quad(a \in \mathbf{C}) \\
& \infty+a=\infty \quad(a \in \widehat{\mathbf{C}}) \\
& a / 0=\infty \quad(a \in \widehat{\mathbf{C}}, a \neq 0) .
\end{aligned}
$$

Entretanto, expressões como $\infty / \infty$ e $\infty-\infty$ não fazem sentido algum. A rigor, deveríamos verificar que essas operações não produzem contradições quando comparadas com as propriedades de $+\mathrm{e} \cdot$, já existentes no plano. Este trabalho, porém, não apresenta qualquer relevância, pois usaremos tais relações apenas para unificar conceitos que envolvem a topologia de $\widehat{\mathbf{C}}$, como, por exemplo, para se definir transformações de Möbius e provar que elas são homeomorfismos de $\mathbf{C}$.

Existe um homeomorfismo especial ${ }^{16} h$ entre $\mathbf{C}$ e a esfera sem o pólo norte, $S^{2} \backslash N$, onde $N=(0,0,1)$. Se colocarmos $h(\infty)=N$, então $h$ torna-se um homeomorfismo entre $\widehat{\mathbf{C}}$ e $S^{2}$. Essa identificação é usada de modo muito freqüente; em vista disso, chamaremos $\widehat{\mathbf{C}}$ de esfera de Riemann. A distinção entre o plano estendido e a esfera de Riemann é apenas verbal, mas ela responde pela motivação intrínseca da grande maioria dos argumentos. A esfera de Riemann herda uma função distância provinda da norma de $\mathbf{R}^{3}$ :

$$
\widehat{d}(z, w)=\|h(z)-h(w)\|
$$

(onde $h$ é o homeomorfismo estabelecido acima); sendo também uma superfície de Riemann compacta, ou seja, é uma variedade complexa e conexa de dimensão 1 (para mais detalhes, ver [36], ou o Apêndice de [16]). Na prática, trabalharemos apenas com dois tipos de sistemas de coordenadas: se $z \in \mathbf{C}$, então este será a identidade definida numa vizinhança $V(z) \subset \mathbf{C}$; e se $z=\infty$, então o sistema de coordenadas $(U, \varphi)$, definido

\footnotetext{
${ }^{16} \mathrm{O}$ inverso da projeção estereográfica.
} 
numa vizinhança $U(\infty)$, será $\varphi(z)=1 / z$. Assim, para cada $z \in \widehat{\mathbf{C}}$, fica determinado um único sistema de coordenadas definido numa vizinhança de $z$, o qual denotaremos por $\varphi_{z}$. Obviamente, 0 deve estar excluído do domínio de $\varphi_{\infty}$.

Duas funções distância $d_{1}$ e $d_{2}$ definidas num mesmo conjunto não-vazio $X$ serão equivalentes quando existir uma constante $C>0$ tal que

$$
\frac{1}{C} d(x, y) \leq d_{1}(x, y) \leq C d_{2}(x, y)
$$

sempre que $x, y \in X$. No caso específico de subconjuntos de $\widehat{\mathbf{C}}$, sabemos que os homeomorfismos locais $\varphi_{z_{0}}$ definem uma função distância

$$
d_{\varphi_{z_{0}}}(z, w)=\left|\varphi_{z_{0}}(z)-\varphi_{z_{0}}(w)\right|
$$

numa vizinhança de $z_{0}$. Tais métricas nos permitem simplificar muitos argumentos envolvendo $\widehat{d}$. Com efeito, temos a

Proposição 1.20 Todo $z_{0} \in \widehat{\mathbf{C}}$ possui uma vizinhança $V\left(z_{0}\right)$ na qual $\varphi_{z_{0}}$ está definida e $d_{\varphi_{z_{0}}}$ é equivalente à $\widehat{d}$. Se $z_{0} \in \mathbf{C}$, então $\varphi_{z_{0}}$ será a identidade, sendo $\widehat{d}$ e $d_{\varphi_{z_{0}}}$ equivalentes sobre qualquer compacto $K$ de $\mathbf{C}$ contendo o ponto $z_{0}$.

DEMONSTRAÇÃo. O argumento é evidente; deixamos a cargo do leitor o trabalho de formalizá-lo.

\section{Derivadas na esfera de Riemann}

Acabamos de ver que cada ponto $z_{0} \in \widehat{\mathbf{C}}$ está contido num sistema de coordenadas pré-fixado, o que nos permite identificar de modo único o plano tangente $T_{z_{0}} \widehat{\mathbf{C}}$ com o próprio $\mathbf{C}$. Em última análise, isso significa que podemos considerar os diferenciais $(d f)_{z}$ como transformações lineares $A$ de $L(\mathbf{C}, \mathbf{C})$, ou mesmo como números complexos, visto que $A$ é sempre da forma $A(z)=\alpha z$. Existe um modo explícito de se relacionar $(d f)_{z} \operatorname{com} A$. Seja $z_{0}$ um ponto arbitrário de $\widehat{\mathbf{C}}$ e consideremos dois sistemas de coordenadas $\left(U, \phi_{1}\right)$ e $\left(V, \phi_{2}\right)$ de $z_{0}$ e $f z_{0}$, respectivamente. Então

$$
\alpha=\left(\phi_{2} f \phi_{1}^{-1}\right)^{\prime} \phi_{1}\left(z_{0}\right)
$$

Em vista dessa igualdade, definiremos $f^{\prime}\left(z_{0}\right)=\alpha$ como sendo a derivada de $f$ no ponto $z_{0}$, que neste caso será sempre um número complexo, e não um diferencial. $\mathrm{O}$ caso em que ambos $z_{0}$ e sua imagem forem diferentes de $\infty$ corresponde à noção usual de derivada do plano complexo.

Resumindo:

$$
\begin{cases}f^{\prime}\left(z_{0}\right)=f_{1}^{\prime}(0), \text { onde } f_{1}(z)=f(1 / z), & \text { se } z_{0}=\infty \text { e } f z_{0} \neq \infty \\ f^{\prime}\left(z_{0}\right)=f_{1}^{\prime}\left(z_{0}\right), \text { onde } f_{1}(z)=1 / f(z), & \text { se } z_{0} \neq \infty \text { e } f z_{0}=\infty \\ f^{\prime}\left(z_{0}\right)=f_{1}^{\prime}(0), \text { onde } f_{1}(z)=1 / f(1 / z), & \text { se } z_{0}=f z_{0}=\infty\end{cases}
$$


Definição. Diremos que uma aplicação $f$ de $\Omega \subset \widehat{\mathbf{C}}$ em $\widehat{\mathbf{C}} e$ analítica quando $f^{\prime}(z)$ existir em todo ponto $z$ de $\Omega$.

Se $f(z)$ for uma função racional, então também o serão quaisquer uma das aplicações $f_{1}$ que servem para definir $f^{\prime}(z)$, o que faz com que toda função racional seja analítica. $\mathrm{Na}$ verdade, elas são as únicas funções analíticas da esfera de Riemann:

Proposição 1.21 Uma aplicação da esfera de Riemann nela mesma será holomorfa ${ }^{17}$ se, e somente se, for um quociente de polinômios, i.é., uma função racional.

\section{Raízes múltiplas}

Os zeros de uma função analítica não identicamente nula $f$, definida num subconjunto aberto do plano e tomando valores em $\mathbf{C}$, formam um subconjunto sem pontos de acumulação em $\mathbf{C}$. Se $z_{0}$ for o único zero de $f$ no disco $\left|z-z_{0}\right|<\delta$, então existe um primeiro inteiro $m \geq 1$ tal que $f^{(m)}\left(z_{0}\right) \neq 0$; chamamos este inteiro de multiplicidade, ou ordem, do zero $z_{0}$. A ordem $m$ de um zero $z_{0}$ é caracterizada como sendo o único inteiro verificando

$$
f(z)=\left(z-z_{0}\right)^{m} g(z),
$$

para alguma função analítica $g(z) \operatorname{com} g\left(z_{0}\right) \neq 0$.

Para zeros de funções analíticas da esfera de Riemann, a definição é similar. Se $(U, \varphi)$ for um sistema de coordenadas sobre o zero $z_{0}$ de $f$, então definimos sua multiplicidade, ou ordem, como sendo a ordem do correspondente zero $\varphi z_{0}$ da função $f \circ \varphi^{-1}$. É claro que se $z_{0}$ for finito, então esta definição coincidirá com a dada anteriormente, desde que tomemos $\varphi$ com sendo a identidade. ${ }^{18}$ (Essa definição de ordem é a mesma que daremos no contexto das funções racionais, o que poderá ser verificado diretamente a partir das propriedades que enunciamos até o momento.)

Obviamente, qualquer ponto $w_{0} \neq 0, \infty$ pode ser descrito através de dois sistemas de coordenadas $w=\phi(z): w=z$ ou $w=1 / z$. Podemos calcular diretamente as derivadas sucessivas de $g(w)=f(1 / w)$ em $w_{0}=1 / z_{0}$ e verificar que $f^{(m)}\left(z_{0}\right)$ será a primeira derivada não-nula de $f$ em $z_{0}$ se, e somente se, $g^{(m)}\left(w_{0}\right)$ for a primeira derivada nãonula de $g$ em $w_{0}$; o que mostra que a definição de multiplicidade de uma raíz independe da escolha do sistema de coordenadas.

Podemos, também, definir a multiplicidade de uma raiz $z_{0}$ da equação $f(z)=w$. Se $w$ for finito, então esta será a multiplicidade de $z_{0}$ como zero da função $f(z)-w$; no caso em que $w=\infty$, este valor será a multiplicidade de $z_{0}$ como zero de $1 / f(z)$. (Recordemos que os pólos de uma função analítica $f \not \equiv \infty$ são sempre isolados). É comum chamarmos esta multiplicidade de número de pré-imagens (com multiplicidade) do ponto $w$.

\footnotetext{
${ }^{17}$ Holomorfia e Analiticidade são sinônimos no caso complexo.

${ }^{18}$ Veremos adiante que esta propriedade vale mesmo que tomemos $\varphi=1 / z$.
} 


\section{Contando raízes}

Chamaremos de disco qualquer subconjunto $\Delta$ da esfera de Riemann que se apresente nas formas $|z|>R$ ou $\left|z-z_{0}\right|<R$, para $z_{0} \neq \infty$. Naturalmente, $\infty$ é o centro primeiro disco, e $z_{0}$ o centro do segundo. A notação $\partial \Delta^{+}$indica que consideramos o bordo de $\Delta$ como uma curva parametrizada no sentido positivo. Como $w=1 / z$ é uma transformação de Möbius, fica fácil ver que qualquer sistema de coordenadas locais $(U, \varphi)$ de $\widetilde{\mathbf{C}}$ transforma discos $\Delta$ em discos $\widetilde{\Delta}, \operatorname{com} \varphi(\partial \Delta)=\partial \widetilde{\Delta}$.

Se $\Delta$ for um disco contido no sistema de coordenadas $(U, \varphi)$, e $f$ for uma função analítica, a valores em $\mathbf{C}$ e definida numa região que contém $U$, então a integral

$$
\frac{1}{2 \pi i} \int_{\partial \widetilde{\Delta}^{+}} \frac{\left(f \circ \varphi^{-1}\right)^{\prime}(z)}{f \circ \varphi^{-1}(z)} d z
$$

nos fornecerá o número de raízes de $f \circ \varphi^{-1}$ dentro do disco $\widetilde{\Delta}$, desde que contemos cada raiz o número de vezes que sua multiplicidade indicar. Notemos, todavia, que a multiplicidade de um zero $z_{0} \in U$ de $f$ é, por definição, a multiplicidade do zero $\varphi z_{0}$ da função $f \circ \varphi^{-1}$; e como $\varphi$ estabelece uma correspondência biunívoca entre os discos, o valor dado pela integral anterior é, de fato, o número de zeros (com mult.) de $f$ no interior do disco $\Delta$.

\subsubsection{Famílias normais}

Recordaremos algumas noções de convergência uniforme local, que usaremos para definir famílias normais. Se $Y$ for um espaço métrico e $X$ um espaço topológico localmente compacto e segundo enumerável - em particular, $\sigma$-compacto - então definimos $C(X, Y)$ como o conjunto de todas as funções contínuas de $X \operatorname{em} Y$. Daremos uma topologia para este conjunto impondo que uma base local de qualquer $f \in C(X, Y)$ consiste de todos os conjuntos

$$
N_{K, \varepsilon}(f)=\left\{g \in C(X, Y): \sup _{x \in K}|f(x)-g(x)|<\varepsilon\right\},
$$

onde $K$ é um compacto de $X$ e $\varepsilon>0$. Chamaremos essa topologia de topologia da convergência uniforme local. Ela não depende da métrica de $Y$, mas apenas da topologia gerada pela métrica deste conjunto (ver [34], pág. 30). Quando $f_{n} \in C(X, Y)$ convergir para uma $f \in C(X, Y)$ nessa topologia, diremos que $f_{n}$ converge uniformemente localmente para $f$ sobre $X$.

Teorema 1.25 A topologia da convergência uniforme local sobre $C(X, Y)$ é metrizável. Se uma sequência $f_{n} \in C(X, Y)$ convergir para uma $f \in C(X, Y)$ nessa topologia, então

(i) $\left.f_{n}\right|_{K}$ converge uniformemente para $\left.f\right|_{K}$, para todo compacto $K \subset X$; e

(ii) Todo $x \in X$ possui uma vizinhança $U$ onde $\left.\left.f_{n}\right|_{U} \rightarrow f\right|_{U}$ uniformemente. Reciprocamente, as condições $(i)$ e (ii) são equivalentes; e sempre que $f_{n}$ satisfizer alguma delas, $f_{n}$ deverá convergir uniformemente localmente para $f$ sobre $X$. 
DEMONSTRAÇÃO. Ver [34], pág. 31.

Agora já temos as ferramentas necessárias para definir quando um conjunto de funções é normal. Se U for um subconjunto aberto e conexo de $\widehat{\mathbf{C}}$ (a conexidade será de fundamental importância), então uma família $\mathscr{F}$ de funções holomorfas definidas em $U$ e tomando valores em $\widehat{\mathbf{C}}$ será dita normal quando seu fecho $\overline{\mathscr{F}}$ em $C(X, Y)$ for um subconjunto compacto. Como $C(X, Y)$ é metrizável, isso se traduz na seguinte sentença:

Normalidade (I). $\mathscr{F}$ será normal se, e somente se, toda sequência de funções $f_{n} \in \mathscr{F}$ possuir uma subsequência convergindo localmente uniformemente sobre $U$.

Notemos que, pelo Teorema 1.28, todo limite $f$ de uma sequência como acima deve ser holomorfa, bem como o fecho $\overline{\mathscr{F}}$, onde qualquer função também deve ser holomorfa.

Uma abordagem um pouco diferente pode ser dada no caso em que $\mathscr{F}$ é uma família de função analíticas ainda definidas em $U$, mas tomando valores em $\mathbf{C}$ e vez de $\widehat{\mathbf{C}}$. A definição de normalidade é a mesma, mas devemos considerar a possibilidade de termos uma sequência $f_{n} \in \mathscr{F}$ convergindo localmente uniformemente sobre $U$ para uma função holomorfa $f$, com $f\left(z_{0}\right)=\infty$, para algum $z_{0} \in U$. A função $f(z)$ assume valores arbitrariamente grandes para $z$ suficientemente próximo de $z_{0}$, enquanto que $f_{n}$ converge uniformemente (com respeito à $\widehat{d}$ ) para $f$ numa vizinhança $V\left(z_{0}\right) \subset U$. Tendo-se em vista tais propriedades, podemos tomar $V$ suficientemente pequena, de modo a termos

$$
\left|f_{n}(z)\right| \geq M
$$

sempre que $z \in V$ e $n \geq n_{0}$, para algum $n_{0}$.

Tomando-se um sistema de coordenadas $\varphi_{z_{0}}$ e trocando-se $f$ por $f \varphi_{z_{0}}$, se necessário, podemos assumir que $z_{0}$ é diferente de $\infty$, e que $V \subset \mathbf{C}$. Queremos mostrar que $f(z)$ é identicamente $\infty$ sobre $V$. Para tanto, usaremos o Teorema de Hurwitz.

A Proposição 1.20 nos permite concluir que a sequência de funções analíticas $1 / f_{n}$ converge uniformemente para $1 / f$ sobre $V$; mas $1 / f_{n}$ nunca se anula, enquanto que $1 / f$ se anula no ponto $z_{0}$. Logo, $1 / f$ é identicamente nula sobre $V$, como afirmamos. Segue daí que o conjunto dos pontos de $U$ onde $f=\infty$ é ao mesmo tempo aberto e fechado (e não-vazio). Como $U$ é conexo, concluímos que $f=\infty$ sobre $U$.

Assim, no caso em que o contradomínio de todas das funções de $\mathscr{F}$ exclui o ponto $\infty$, temos uma caracterização alternativa de

Normalidade (II). $\mathscr{F}$ será normal se, e somente se, toda sequência infinita $f_{n} \in \mathscr{F}$ possuir uma subsequência $f_{n_{k}}$ satisfazendo alguma das seguintes propriedades:

(a) $f_{n_{k}}$ converge localmente uniformemente sobre $U$ para uma função $f \in C(U, \mathbf{C})$;

(b) $f_{n_{k}}$ diverge localmente para $\infty$, no sentido de que, dados quaisquer compactos $K_{1} \subset \mathbf{C}$ e $K_{2} \subset U$, existe um natural $k_{0}$ tal que f $K_{2}$ está no complementar de $K_{1}$, para todo $k \geq k_{0}$. 


\subsubsection{Resultados gerais sobre funções analíticas}

Reuniremos, neste tópico, algumas propriedades sobre funções analíticas, muitas das quais sem conexão direta umas com as outras.

Teorema 1.26 (Lema de Schwarz) Se $f(z)$ for analítica para $|z|<1$ e satisfizer as condições $|f(z)| \leq 1$ e $f(0)=0$, então $|f(z)| \leq|z|$ e $\left|f^{\prime}(0)\right| \leq 1$. A igualdade nas últimas duas expressões ocorrerá somente no caso em que $f(z)=c z$, com $|c|=1$.

DEMONSTRAÇÃo. Ver [1], pág. 135.

Teorema 1.27 (Schwarz-Pick) Seja $f(z)$ uma aplicação holomorfa do disco unitário $|z|<1$ nele mesmo. Então

$$
\left|\frac{f(z)-f(w)}{1-f(z) \overline{f(w)}}\right| \leq\left|\frac{z-w}{1-z \bar{w}}\right|
$$

para quaisquer ze $w$.

DEMONSTRAÇÃo. Ver pág 11 de [16].

Existem diversas adaptações do Teorema de Schwarz-Pick. Apresentaremos uma para funções analíticas que levem o disco unitário $\Delta=\{|z|<1\}$ no semiplano esquerdo $H_{\ell}=\{\operatorname{Re} z<0\}$. Constitui fato bem conhecido que o homeomorfismo da esfera de Riemann

$$
w=\frac{z-i}{z+i}
$$

também chamado de trasformação de Cayley, leva o semiplano positivo $\operatorname{Im} z>0$ sobre o disco unitário $\Delta$. Compondo com uma rotação, vemos que a imagem de $H_{\ell}$ pela transformação de Möbius

$$
R(\zeta)=\frac{\zeta+1}{\zeta-1}
$$

é exatamente $\Delta$. Para simplificar a notação, colocaremos

$$
[z, w]=\frac{z-w}{1-z \bar{w}}
$$

observando que

$$
[R(\zeta), R(\eta)]=\left(\frac{\bar{\eta}-1}{\eta+1}\right)\left(\frac{\zeta-\eta}{\zeta+\bar{\eta}}\right) .
$$

Agora sabemos que a função $R f(z)$ está nas condições do Teorema de Schwarz-Pick, de onde concluímos que

$$
\left|\frac{f(z)-f(w)}{f(z)+\overline{f(w)}}\right|=|[R f(z), R f(w)]| \leq|[z, w]| .
$$

Temos, assim, o seguinte 
Corolário 1.2 Seja $f(z)$ uma função holomorfa definida no disco unitário aberto $\Delta$, tomando valores em $H_{\ell}$. Então

$$
\left|\frac{f(z)-f(w)}{f(z)+\overline{f(w)}}\right| \leq\left|\frac{z-w}{1-z \bar{w}}\right|,
$$

quaisquer que sejam $z, w \in \Delta$.

Teorema 1.28 (Weierstrass) Seja $f_{n}$ uma sequência de funções holomorfas definidas num aberto $U \subset \mathbf{C}$, tomando valores em $\mathbf{C}$. Se $f_{n}$ convergir uniformemente para uma função $f$, então $f$ será holomorfa e todas as sequências de derivadas $f_{n}^{(j)}$ convergirão uniformemente sobre compactos $K \subset U$ para $f^{(j)}$.

DEMONSTRAÇÃO. Ver [34], pág. 4.

Teorema 1.29 (Hurwitz) Seja $f_{n}$ uma sequência de funções complexas e analíticas, definidas num suconjunto aberto e conexo $\Omega$ de $\mathbf{C}$. Suponhamos que $f_{n}$ nunca se anule sobre $\Omega$, para todo $n$. Se $f_{n}$ convergir uniformemente para uma função $f$ sobre $\Omega$, então esta deverá ser ou identicamente nula, ou $f(z) \neq 0$, para todo $z \in \Omega$.

DemonstraÇão. Ver [1], pág. 176.

\section{Teorema da Curva de Jordan}

Recordemos algumas definições. Qualquer função contínua $\zeta$ definida num intervalo fechado $[a, b]$ é chamada de curva. O contradomínio de $\zeta$ pode ser qualquer espaço topológico $X$, mas o nosso ${ }^{19}$ interesse aqui é a esfera de Riemann $\widehat{\mathbf{C}}$. Diremos que uma curva $\zeta$ é fechada se $\zeta(a)=\zeta(b)$; e que é simples se não tiver auto-interseções. De um modo mais preciso, $\zeta$ será simples quando a restrição de $\zeta$ aos intervalos $(a, b]$ e $[a, b)$ for injetora, o que não exclui a possibilidade de $\zeta$ ser fechada. Agora apresentaremos o Teorema da Curva de Jordan, um resultado fundamental de Topologia, fácil de enunciar mas com uma demonstração muito elaborada. Como a natureza deste teorema é topológica, vamos abandonar a estrutura diferenciável e algébrica de $\widehat{\mathbf{C}}$, trocando este espaço pela sua cópia homeomorfa $S^{2} \subset \mathbf{R}^{3}$.

Teorema 1.30 (da Curva de Jordan) Seja $\zeta$ uma curva fechada e simples contida em $S^{2}$. Então o complemento $S^{2} \backslash \zeta$ consiste de apenas duas componentes conexas $W_{1} e$ $W_{2}$, com

$$
\partial W_{1}=\partial W_{2}=\zeta .
$$

DEMONSTRAÇÃO. Ver [35], pág. 383.

Uma curva fechada e simples é também conhecida como curva de Jordan. Podemos usar este resultado para se definir o interior $I(\zeta)$ de uma curva de Jordan $\zeta$ contida

\footnotetext{
${ }^{19}$ Denotaremos a imagem de uma curva $\zeta$ com o mesmo símbolo $\zeta$ e, quando o seu contradomínio for $X$, diremos que $\zeta$ está contida em $X$.
} 
em C. Considerando-a como uma curva em $\widehat{\mathbf{C}}$, o ponto no infinito $\infty$ está em alguma das componentes conexas do complementar de $\zeta$, a qual chamamos de componente ilimitada. A outra componente - pois existem apenas duas - é chamada de interior de $\zeta$. 


\section{Dinâmica Complexa}

Ao contrário do que indica o título deste Capítulo, não faremos um estudo abrangente de Dinâmica Complexa, mas apenas descreveremos os objetos principais. Objetivamos enunciar alguns resultados que usaremos nos Capítulos seguintes. Em nível crescente de complexidade, indicamos as seguintes referências ao leitor interessado em aprofundar seus estudos sobre o assunto:

$$
\text { [34] [9] [16] [33]. }
$$

\section{Conceitos básicos de dinâmica}

Consideremos um subconjunto aberto $\Omega$ da esfera de Riemann, onde está definida uma função analítica $f$, tomando valores em $\widehat{\mathbf{C}}$.

Dado $z_{0} \in \Omega$, definimos os iterados do ponto $z_{0}, f^{n} z_{0}$, indutivamente por $f^{0} z_{0}=z_{0}$ e $f^{n+1} z_{0}=f\left(f^{n} z_{0}\right)$. Se $U$ for um subconjunto de $\Omega$ tal que $f U \subset U$, então diremos que $U$ é um conjunto invariante; o fato de $U$ ser invariante se traduz no seguinte: para todo $z_{0} \in U$, seus iterados $f^{n} z_{0}$ estão bem definidos. Para enfatizar que $U$ é invariante, em muitos casos escreveremos $(f, U)$, e também diremos que $f$, ou $(f, U)$, é um sistema dinâmico. ${ }^{1}$

Um dado $z_{0} \in \Omega$ será um ponto fixo de $f$ quando $f z_{0}=z_{0}$; e um ponto periódico de período $p \geq 1$ quando $z_{0}$ for ponto fixo de $f^{p}$. Observemos, deste modo, que um ponto periódico possui vários períodos diferentes e que, quando dizermos que $z_{0}$ tem período $p$, não necessariamente isso significa que $p$ seja o menor período também chamado de período minimal. Muitos autores adotam o período minimal como definição período.

Seja $z_{0}$ um ponto fixo de $f$. Definimos o multiplicador de $f$ no ponto $z_{0}$ como sendo $\lambda=f^{\prime}\left(z_{0}\right) .{ }^{2}$ Diremos que $z_{0}$ é repulsor, indiferente, atrator, ou superatrator, precisamente nos casos em que o módulo do multiplicador $|\lambda|$ for estritamente maior que 1 , igual a 1 , estritamente menor que 1 , ou igual a zero, respectivamente. Um ponto fixo atrator será geometricamente atrator quando seu multiplicador for não-nulo.

\footnotetext{
${ }^{1}$ Enfatizamos, porém, que a noção do que deva ser um sistema dinâmico é muito mais ampla e intuitiva e, muitas vezes, uma formalização restritiva torna-se incoveniente e desnecessária.

${ }^{2}$ Ver tópico que define $f^{\prime}(\infty)$.
} 
Idêntica terminologia se aplica à pontos periódicos; por exemplo, um ponto periódico $z_{0}$ de período $p$ será chamado de atrator quando $z_{0}$ for um ponto fixo atrator de $f^{p}$. $\mathrm{O}$ conjunto de iterados de $f^{n} z_{0}$ de um ponto $z_{0}$, contando-se inclusive o ponto inicial $z_{0}$, é chamado de órbita de $z_{0}$. A órbita de um ponto periódico é chamada de órbita periódica ou ciclo. Quando escrevermos $z_{0}, \ldots, z_{p-1}$ para indicar um ciclo, ficará subentendido que $f z_{i}=z_{i+1}$ e que $p$ é o período minimal. Podemos empregar a mesma terminologia que usamos no caso de pontos periódicos para se definir ciclos atratores, indiferentes, etc. Definimos o mutiplicador de um ciclo $z_{0}, \ldots, z_{p-1}$ de período $p$ como sendo a derivada de $f^{p}$ em qualquer um dos pontos do ciclo; de fato, estas derivadas serão sempre iguais a

$$
\prod_{i=0}^{p-1} f^{\prime}\left(z_{i}\right),
$$

o que pode ser verificado a partir da regra da cadeia (o caso em que um dos pontos é $z_{i}=\infty$ envolve o uso de cartas; ver seção sobre derivadas na esfera de Riemann).

\section{A dinâmica de pontos fixos atratores e repulsores}

Seja $z_{0}$ um ponto fixo atrator de $f$, com multiplicador $\lambda$ satisfazendo $|\lambda|<\rho<1$. Suponhamos, primeiramente, que $z_{0}$ seja finito. Então segue da regra da cadeia que existe um vizinhança invariante $\Delta\left(z_{0}, \varepsilon\right)$ do ponto $z_{0}$, na qual

$$
\left|f^{n} z-z_{0}\right| \leq \rho^{n}\left|z-z_{0}\right|
$$

o que faz com que os iterados $f^{n} z$ de qualquer $z$ em $\Delta\left(z_{0}, \varepsilon\right)$ convirjam exponencialmente para o ponto fixo.

Um dos modos de se entender o comportamento de um sistema dinâmico é via conjugações. Sejam $(f, U)$ e $(g, V)$ dois sistemas dinâmicos. Diremos que uma aplicação sobrejetiva $\varphi$ de $U$ em $V$ conjuga $f$ à $g$, ou que $\varphi$ é uma conjugação de $f$ para $g$, quando

$$
\varphi(f z)=g \varphi(z)
$$

sobre $U$. Se $U$ e $V$ forem regiões do plano, e se $\varphi$ for uma equivalência conforme, então diremos que $\varphi$ é uma conjugação conforme, ou que $f$ e $g$ estão conformemente conjugados.

Voltando à análise local de $f$ perto de um ponto fixo atrator $z_{0}$, podemos usar o conceito de conjugação para contornar o caso em que $z_{0}=\infty$; ele é reduzido ao anterior através da mudança de coordenadas $w=1 / z$, que conjuga $f$ à um sistema dinâmico definido numa vizinhança de 0 , com um ponto fixo atrator neste ponto.

Definimos a bacia de atração $A\left(z_{0}\right)$ do ponto fixo $z_{0}$ como sendo o conjunto de todos os pontos de $\Omega$ cujos iterados estão bem definidos e convergem para $z_{0}$. Se um ponto $z$ de $\Omega$ tiver algum iterado $f^{n_{0}} z$ em $\Delta\left(z_{0}, \varepsilon\right)$, então ele será atraído para $z_{0}$, de modo que, para todo inteiro não-negativo $k$, 


$$
A\left(z_{0}\right)=\bigcup_{n \geq k} f^{-n} \Delta\left(z_{0}, \varepsilon\right)
$$

A igualdade acima continua válida para $z_{0}=\infty$, mas, neste caso, devemos trocar $\Delta\left(z_{0}, \varepsilon\right)$ por algum disco $|z|>R$ centrado no $\infty, \operatorname{com} R$ suficientemente grande.

Definimos a bacia de atração de um ciclo atrator $C: z_{0}, \ldots, z_{p-1}$ como sendo a reunião das bacias de atratação $A\left(z_{i}, f^{p}\right)$ dos pontos fixos atratores $z_{i}$ de $f^{p}$. É fácil ver que

$$
f A\left(z_{i}, f^{p}\right) \subset A\left(z_{i+1}, f^{p}\right),
$$

e que pontos suficientemente próximos de $C$ são atraídos numa órbita "quase periódica" a medida que o número de iterados crescem, cujo $\omega$-limite é o próprio $C$. Recordemos que o $\omega$-limite de uma órbita $f^{n} z_{0}$, não necessáriamente periódica, é o conjunto dos limites subsequenciais da sequência $z_{n}=f^{n} z_{0}$.

Seja $z_{0} \neq \infty$ um ponto fixo geometricamente atrator, com multiplicador $\lambda$. Como $f$ é analítica, temos

$$
f(z)=z_{0}+\lambda\left(z-z_{0}\right)+a_{p}\left(z-z_{0}\right)^{p}+\cdots
$$

numa vizinhança de $z_{0}$. É de se esperar, portanto, que para $z$ suficientemente próximo de $z_{0}$, a dinâmica de $f(z)$ seja conjugada à da aplicação $g(\zeta)=\lambda \zeta$. De fato, esta questão já foi resolvida (1884) por G. Koenigs:

Teorema 2.1 Seja zo um ponto fixo geometricamente atrator de $f$ (podendo inclusive $\left.z_{0}=\infty\right)$, com multiplicador $\lambda$. Então existem vizinhanças conexas $V\left(z_{0}\right)$ e $U(0)$, e uma equivalência conforme $\varphi$ entre estas regiões conjugando $(f, V) \grave{a}(g, U)$. Qualquer outra conjugação é um fator constante c $\varphi$ de $\varphi$.

Referência: [9], página 31.

Deste modo, não importa qual seja a forma de $f$, sabemos que a menos de uma mudança de coordenadas (conjugação conforme), a dinâmica de $f$ perto de um ponto fixo atrator se comporta como uma rotação seguida de uma contração, e que quaisquer iterados convergem exponencialmente para o ponto fixo atrator numa espiral.

\section{Pontos fixos repulsores}

O caso de pontos fixos repulsores também pode ser deduzido a partir do Teorema acima. De fato, seja $\lambda_{0}$ o multiplicador de um ponto fixo repulsor $z_{0}$. Segue do Teorema da função inversa que existem vizinhanças conexas $U\left(z_{0}\right)$ e $V\left(z_{0}\right)$ tais que $f$ é uma aplicação univalente de $U$ sobre $V$. Em particular, a inversa local $f^{-1}$ de $f$ existe e define uma função analítica sobre $V$, com um ponto fixo atrator em $z=z_{0}$, cujo multiplicador é $\lambda_{1}=1 / \lambda_{0}$. Podemos escolher $V$ suficientemente pequeno de modo que exista uma equivalência conforme $\varphi$ conjugando $\left(f^{-1}, V\right)$ à $\left(\lambda_{1} \zeta, W\right)$, onde $W$ é uma vizinhança conexa de 0 ; além disso, podemos tomar $V$ de modo que $V \subset U$. Fica fácil ver que $\varphi(f z)=\lambda_{0} \varphi(z)$ sobre $U$. Observemos que $U$ não é invariante por $f$. 


\subsection{Iteração de Funções Racionais}

\section{O Método de Newton}

Um dos problemas básicos de Análise Numérica é o de se determinar algoritmos que aproximem as raízes de um polinômio. Um argumento simples envolvendo tangentes do gráfico de um polinômio real $y=P(x)$ nos fornece um algoritmo baseado na iteração da função

$$
f(x)=x-\frac{P(x)}{P^{\prime}(x)},
$$

conhecido como método de Newton. A idéia é que para valores iniciais $x_{0}$ próximos de uma raíz do polinômio, a sequência de iterados $f^{n} x_{0}$ deve convergir para esta raíz. Podemos estender este método para o plano complexo, usando a mesma fórmula (2.2) para se definir $f(z)$ a partir de um polinômio complexo $P(z)$.

O método de Newton data de 1690 (J. Raphson), e questões simples relacionadas à sua convergência nos conduzem de modo natural à importantes problemas de Dinâmica Complexa, como o de se determinar bacias de atração de pontos fixos, ou mesmo de entender como se dá a convergência do método, o que pode ser feito via conjugações. Notemos, então, que um estudo adequado do método de Newton toma por base propriedades da família $f^{n}$ de funções racionais.

\section{Funções Racionais}

Se $P(z)$ e $Q(z)$ forem polinômios não-nulos, então chamaremos o quociente

$$
f(z)=\frac{P(z)}{Q(z)}
$$

de função racional. Os zeros de $Q$ são as singularidades desta função, que serão apenas de dois tipos: pólos ou singularidades removíveis. O último caso consiste dos zeros em comum de $P$ e $Q$, com mesma multiplicidade. De qualquer modo, podemos concelar os fatores que aparecem em ambos os polinômios e tomá-los sem zeros em comum, $o$ que sempre faremos de agora em diante. Deste modo, todas as raízes de $Q$ são pólos de $f(z)$. Definimos a multiplicidade de um polo $z_{0}$ como sendo a multiplicidade de $z_{0}$ como zero da função $Q(z)$. Podemos atribuir os valores

$$
f\left(z_{0}\right)=\infty \text { e } f(\infty)=\lim _{z \rightarrow \infty} f(z)
$$

no caso em que $z_{0}$ for um pólo, o que torna $f$ uma aplicação contínua da esfera de Riemann nela mesma. Se $\infty$ for um zero (ou um pólo) de $f(z)$, então definimos sua multiplicidade como sendo a multiplicidade do zero (respect. pólo) $z=0$ da função racional $f(1 / z)$. Pode-se demonstrar que ${ }^{3}$

\footnotetext{
${ }^{3}$ Os seguintes resultados podem ser encontrados em [1].
} 
Proposição 2.1 Uma função racional $f(z)$ possui um igual número de pólos e raízes em $\widehat{\mathbf{C}}$, desde que eles sejam contados com multiplicidade. Este número é igual ao maior dos graus $\partial P, \partial Q$.

Definimos o valor comum dado pela proposição acima como sendo o grau $\partial f$ da função racional $f$. Este princípio nos permite concluir que cada ponto $w$ de $\mathbf{C}$ possui exatamente $\partial f$ pré-imagens (contando-se multiplicidade), visto que $f(z)-w$ é uma função racional cujos pólos são os mesmos de $f(z)$. No caso em que $w=\infty$ este argumento deve ser modificado; basta notar que as pré-imagens de $\infty$ são os pólos de $f(z)$. Existe, ainda, um meio mais direto de se calcular o grau de uma função racional, onde não precisamos nos preocupar com multiplicidades:

Proposição 2.2 Exceto para um número finito de exceções, para todo $c \in \widehat{\mathbf{C}}$ as raízes da equação $f(z)=c$ são sempre distintas.

As funções racionais de grau 1 são chamadas de transformações de Möbius, e são responsáveis pela caracterização geométrica de $\widehat{\mathbf{C}}$. Toda transformação de Möbius é um homeomorfismo da esfera de Riemann. Recordaremos agora algumas propriedades destas transformações, que são sistematicamente discutidas em [1], páginas 76-82.

No plano complexo estendido $\widehat{\mathbf{C}}$, trataremos retas e círculos com o mesmo nome: doravante, todos serão chamados de círculos. Isso porque, do ponto de vista das trasformações de Möbius, estes objetos são inestinguíveis:

Proposição 2.3 A imagem de um círculo por uma transformação de Möbius é sempre um círculo.

O ponto no infinito $\infty$ serve, então, para diferenciar os círculos que são retas dos círculos propriamente ditos, i.é., os círculos limitados. Toda reta passa por $\infty$.

Proposição 2.4 Dados três pontos distintos $z_{0}, z_{1}$ e $z_{2}$ de $\widehat{\mathbf{C}}$, existe uma única transformação de Möbius que leva estes pontos em 1,0 e $\infty$, respectivamente.

Como a composição de duas transformações de Möbius ainda é uma transformação de Möbius, a propriedade acima vale para quaisquer três pontos distintos no lugar de 1,0 e $\infty$. Pode-se demonstrar, ainda, que se $M$ for uma transformação de Möbius e $f(z)$ uma aplicação racional, então

Proposição $2.5 \partial M \circ f=\partial f \circ M$.

Seja $C$ um círculo e consideremos dois pontos $z$ e $z^{*}$ de $\widehat{\mathbf{C}}$. Quando $\overline{T z^{*}}=T z$, para alguma transformação de Möbius que leve $C$ no eixo real, então diremos que $z$ e $z^{*}$ são simétricos. Essa noção independe da transformação $T$, ou seja, dois pontos que forem simétricos com relação à uma certa transformação, o serão com relação à qualquer transformação de Möbius.

Proposição 2.6 (Princípio de simetria) Toda transformação de Möbius T leva um par de pontos simétricos com relação à um círculo $C$ noutro par de pontos simétricos com relação à $T(C)$. 
Dados dois pontos $z_{0}$ e $z_{1}$ de $\widehat{\mathbf{C}}$, denotamos por $L\left(z_{0}, z_{1}\right)$ a reta que passa por estes dois pontos. A reta perpendicular à $L\left(z_{0}, z_{1}\right)$ que passa pelo ponto médio $\left(z_{0}+z_{1}\right) / 2$ será chamada de bisetor dos pontos $z_{0}$ e $z_{1}$; indicá-la-emos por $N\left(z_{0}, z_{1}\right)$.

Pode-se demonstrar que se $C$ for um círculo limitado, de raio $R$ e centro $a$, então o simétrico $z^{*}$ de qualquer $z$ de $\widehat{\mathbf{C}}$ será dado por

$$
z^{*}=\frac{R^{2}}{\bar{z}-\bar{a}}+a ;
$$

em particular, o simétrico de $\infty$ é o centro $a$ do círculo. Se $C$ for uma reta, então o simétrico de todo ponto $z \in C$ será o próprio $z$, enquanto que, para $z$ fora de $C$, $z^{*}$ será o único ponto de $\mathbf{C}$ que torna $C=N\left(z, z^{*}\right)$.

As propriedades geométricas que descrevemos acima nos permitem estabelecer resultados de forma elegante e direta, o que de outra forma seria feito através de cálculos extensos e enfadonhos. O próximo exemplo ilustra o que acabamos de observar.

Exemplo - O método de Newton novamente - Consideremos a função racional

$$
f(z)=z-\frac{P(z)}{P^{\prime}(z)}
$$

onde $P(z)$ é um polinômio de grau dois, com duas raízes distintas $z_{0}$ e $z_{1}$. O bisetor $N\left(z_{0}, z_{1}\right)$ determina dois semiplanos abertos $H_{0}$ e $H_{1}$, contendo $z_{0}$ e $z_{1}$, respectivamente. Observemos que se $P(z)=a z^{2}+b z+c$, então

$$
f(z)=\frac{a z^{2}-c}{2 a z+b} \text { e } f^{\prime}(z)=\frac{2 a P(z)}{\left(P^{\prime} z\right)^{2}}
$$

onde a primeira igualdade vale por toda a esfera de Riemann e a segunda somente em C. Deste modo, $z_{0}$ e $z_{1}$ são pontos fixos atratores de $f$. Mostraremos que, de fato, $H_{0} \mathrm{e}$ $H_{1}$ são as bacias de atração destes dois pontos. Existe apenas um outro ponto fixo de $f$ sobre $\widehat{\mathbf{C}}: \infty$. Usando-se a carta local $w=1 / z$ verifica-se que seu multiplicador é $\lambda=2$; logo, $\infty$ é um ponto fixo repulsor.

Uma conjugação conforme $\varphi$ de $(f, \widehat{\mathbf{C}})$ para $\left(\zeta^{2}, \widehat{\mathbf{C}}\right)$ deve levar pontos fixos em pontos fixos, preservando-se os multiplicadores. Em vista disso, escolhemos $\varphi$ como sendo a transformação de Möbius que leva $z_{0}, z_{1}$ e $\infty$ em $0, \infty$ e 1 , respectivamente. Mostremos que $\varphi \circ f(z)=(\varphi z)^{2}$. Com efeito, $\varphi(f z)$ e $(\varphi z)^{2}$ são funções racionais de grau dois (ver Prop. 2.5), com as mesmas raízes e os mesmos pólos, todos com a mesma multiplicidade. ${ }^{4}$ Logo, os quocientes de polinômios que dão origem a estas duas transformações devem ser iguais, exceto por um fator constante; este fator só pode ser 1 , visto que as duas funções coincidem em $z=\infty$.

O ponto médio $z_{M}=-b /(2 a)$ das raízes é levado por $f$ em $\infty$; $\log o\left(\varphi z_{M}\right)^{2}=1$, de onde concluímos que $\varphi\left(z_{M}\right)=-1$. Portanto, a conjugação $\varphi$ transforma o bisetor $N\left(z_{0}, z_{1}\right)$ num círculo passando pelo ponto -1 ; este círculo dever ser limitado, já

\footnotetext{
${ }^{4}$ A raíz múltipla é $z=z_{0}$ e o pólo $z=z_{1}$. Para provar que $z_{1}$ é um pólo de ordem 2 , coloque $\varphi(z)$ na sua forma explícita, verificando que $\varphi^{\prime}(z)=(a d-b c) /(c z+d)^{2}$.
} 
que $z_{1} \notin N\left(z_{0}, z_{1}\right)$ é o único ponto levado por $\varphi$ em $\infty$. O centro deste círculo é a origem, o que pode ser visto usando-se o princípio de simetria. Com isso, mostramos que $\varphi N\left(z_{0}, z_{1}\right)$ nada mais é que o círculo unitário, e que $\varphi$ transforma os semiplanos abertos $H_{0}$ e $H_{1}$ em $|z|<1$ e $|z|>1$, respectivamente.

\subsection{Conjuntos de Julia e Fatou}

“(...) la structure de $E^{\prime}$ est la même dans toutes ses parties.”

(GASTON JULiA)

Seja $f$ uma função racional de grau maior ou igual a dois. Definimos o conjunto de Fatou, $F(f)$, de $f$ como sendo o conjunto dos pontos para os quais existe uma vizinhança conexa $U$ onde a família de iterados $\left.f^{n}\right|_{U}, n \geq 1$, é normal. O complementar do conjunto de Fatou em $\widehat{\mathbf{C}}$ é chamado de conjunto de Julia de $f$; denotá-lo-emos por $J(f)$. Pode-se mostrar que o conjunto de Julia é não vazio; e como $F$ é, por definição, aberto, segue-se que $J$ é um subconjunto compacto da esfera de Riemann. Pode-se mostrar que ambos os conjuntos de Julia e Fatou são completamente invariantes, ou seja, $f^{-1} J=J$ e $f^{-1} F=F$.

Um ciclo $z_{0}, \ldots, z_{p-1}$, de período $p \geq 1$, será chamado de parabólico quando seu multiplicador $\lambda$ for uma raíz da unidade, i.é., $\lambda^{n}=1$, para algum $n \geq 1$.

Proposição 2.7 O conjunto de Julia $J(f)$ contém todos os ciclos parabólicos e repulsores da aplicação $f$. O fecho destes últimos coincide com o próprio $J(f)$. Além disso, $f$ é topologicamente mixing, ou seja, para todo aberto relativo $V \subset J$ existe um $n \geq 1$ tal que $f^{n} V=J$.

Referência: [9].

A dinâmica de $f$ sobre $J$ é caótica, como indica a Proposição acima. Por outro lado, pelo modo como definimos o conjunto de Fatou, é de se esperar um certo grau de previsibilidade do sistema $(f, F)$. De fato, $f$ leva componente de Fatou sobre componente $^{5}$ de Fatou e, além disso, podemos classificar os diferentes tipos de componentes. Uma componente $U$ do conjunto de Fatou será periódica quando existir $p \geq 1$ tal que $f^{p} U=U$. Qualquer $p$ nas condições especificadas é chamado de período de $U$. Diremos que $U$ é pré-periódica quando existir um $p>1$ tal que $f^{p} U$ é uma componente periódica de $F$.

Uma componente periódica $U$ de período $p$ será chamada de:

\footnotetext{
${ }^{5}$ Componente, aqui, significa componente conexa, as quais serão abertas, pois $F$ é aberto.
} 
(i) Atratora quando existir um ponto periódico atrator $z_{0} \in U$ de período $p$ tal que, para todo $z \in U$, os iterados $f^{n p} z$ convergem para $z_{0}$.

(ii) Parabólica quando existir um ponto periódico parabólico de período $p z_{0} \in \partial U$ tal que os iterados $f^{n p} z$ convergem para $z_{0}$, para todo $z \in U$.

(iii) Disco de Siegel quando $\left(f^{p}, U\right)$ for conjugado à uma rotação irracional $(R, \Delta)$ do disco $\Delta=\{|z|<1\}$ por meio de uma equivalência conforme entre $U$ e $\Delta$.

(iv) Anel de Herman quando $\left(f^{p}, U\right)$ for conjugado à uma rotação irracional $(R, A)$ por meio de uma equivalência conforme entre $U$ e um conjunto $A$ da forma

$$
A=\left\{z \in \mathbf{C}: R_{1}<|z|<R_{2}\right\} .
$$

Definimos

$$
P(f)=\overline{\left\{f^{n} z: n>1, f^{\prime}(z)=0\right\}} .
$$

Teorema 2.2 $P(f)$ contém todos os ciclos atratores de $f$ e todas as fronteiras de Discos de Siegel e Anéis de Herman.

Referência: [33], página 40.

Teorema 2.3 (da Classificação) O número de componetes periódicas do conjunto de Fatou é finito, e toda componente de F é pré-periódica. Uma componente periódica do conjunto de Fatou é sempre de algum dos tipos (i)-(iv) especificados acima.

Referências: [9]; ou [33], página 37.

\subsubsection{Hiperbolicidade}

Uma métrica Riemanniana $\langle\cdot, \cdot\rangle_{z}$ definida num aberto não-vazio da esfera de Riemann será dita conforme quando existir uma função analítica real $\gamma(z)>0$ definida nesta vizinhança, tal que, identificando-se $T_{z} \widehat{\mathbf{C}}=\mathbf{C}$, tenhamos

$$
\langle 1, i\rangle_{z}=0,\langle i, i\rangle_{z}=\langle 1,1\rangle_{z}=\gamma(z)^{2} \text {. }
$$

Uma função racional $f$ de grau $\geq 2$ será chamada de hiperbólica quando existir uma métrica conforme $\mu$ definida numa vizinhança de $J(f)$ tal que, para algum $\eta>1$,

$$
\|D f(z) \cdot v\|_{\mu}>\eta\|v\|_{\mu}
$$

para todo $z \in J$ e $v \in T_{z} \widehat{\mathbf{C}}$. Pode-se demonstrar que, sendo $f$ hiperbólica, existirão constantes $C>0$ e $\alpha>1$ tais que

$$
\left\|D f^{n}(z) \cdot v\right\| \geq C \alpha^{n}\|v\|
$$

para todo $z \in J$ e $v \in T_{z} \widehat{\mathbf{C}}$, onde $\|\cdot\|$ é a métrica esférica de $\widehat{\mathbf{C}}$ (ver [33], pág. 45). No caso em que $J$ excluir o ponto no infinito, i.é., $J \subset \mathbf{C}$, existirá uma constante $C_{0}>0$ tal que 


$$
\left|\left(f^{n}\right)^{\prime}(z)\right| \geq C_{0} \alpha^{n}
$$

para todo $n \geq 1$ e $z \in J$.

Usando a compacidade de $J$, podemos estender a desigualdada acima para um aberto contendo $J$.

Teorema 2.4 Uma função racional $f$, de grau $\geq 2$, será hiperbólica precisamente quando $P(f)$ for disjunto de $J(f)$.

Referência: [33], página 45.

Proposição 2.8 Os ciclos de uma função racional hiperbólica são de apenas dois tipos: atratores ou repulsores.

DEMONSTRAÇÃO. Suponhamos que $z_{0}$ seja um ponto periódico indiferente de período p. O ciclo $z_{0}, f z_{0}, \ldots, f^{p-1} z_{0}$ deve estar contido no conjunto de Fatou de $f$, pois, caso contrário, pelo Teorema 2.2, ambos $P(f)$ e $J(f)$ teriam como subconjunto em comum todo o ciclo, o que contradiz o fato de $J(f)$ ser hiperbólico (ver Teo. 2.4). Além disso, as componentes periódicas do conjunto de Fatou (ver o Teorema da Classificação) são de apenas um tipo: atratoras. De fato, os pontos periódicos parabólicos - que estão todos em $J(f)$, pela Prop. 2.7 - neste caso não existem; além disso, também não existem discos de Siegel, tampouco anéis de Herman, visto que os bordos destes conjuntos são subconjuntos não-vazios de ambos $P(f)$ e $J(f)$. Pelo Teorema da Classificação, o ponto $z_{0}$ deve ser atraído para um ciclo atrator; mas isso faz com que a própria órbita periódica de $z_{0}$ seja o ciclo atrator, e concluímos daí que não podem existir ciclos indiferentes.

\subsection{Figuras}

Nesta seção, apresentaremos algumas figuras de conjuntos de Julia de funções quadráticas $z^{2}+c$. Elas foram feitas através Software JOMagic, disponível na web. As figuras do lado esquerdo são conjuntos de Julia ${ }^{6}$, enquanto que o ponto amarelo do lado direito indicará o respectivo valor do parâmetro $c$. O conjunto (em preto) que aparece do lado direito é o famoso conjunto de Mandelbrot $\mathscr{M}$ associado à família quadrática. Um valor de $c$ vai estar neste conjunto precisamente quando o conjunto de Julia cheio $K_{c}$ de $z^{2}+c$ for conexo (por definição, $z$ está em $K_{c}$ quando seus iterados não divergirem para infinito; este é um subconjunto compacto do plano e sua fronteira é igual ao conjunto de Julia de $z^{2}+c$ ). Pode-se mostrar que o complementar da fronteira de $\mathscr{M}$ é precisamente o conjunto dos parâmetros onde a família quadrática é $J$-estável. Além disso, sabe-se que no cardióide principal - imagem de $|z|<1 / 2$ por $f(z)=z(1-z)$ - indicado na figura, a família é hiperbólica; logo, usando os resultados do último capítulo, vemos que a dimensão de Hausdorff da grande maioria dos conjuntos apresentados logo mais varia analiticamente (real) com respeito à $c$.

\footnotetext{
${ }^{6}$ Mais precisamente, as figuras em preto são os conjuntos de Julia cheio $K_{c}$ que definiremos logo adiante.
} 

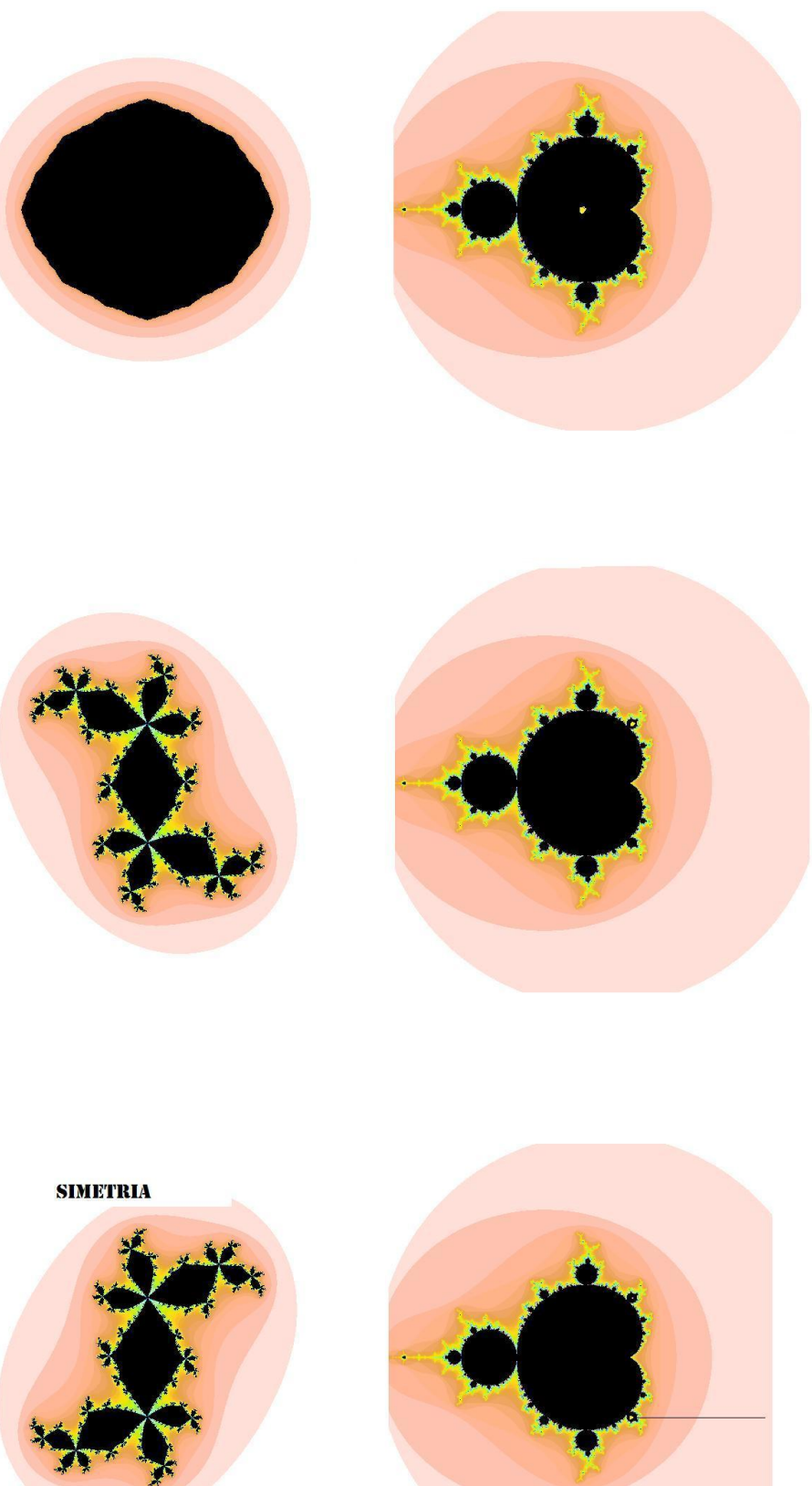

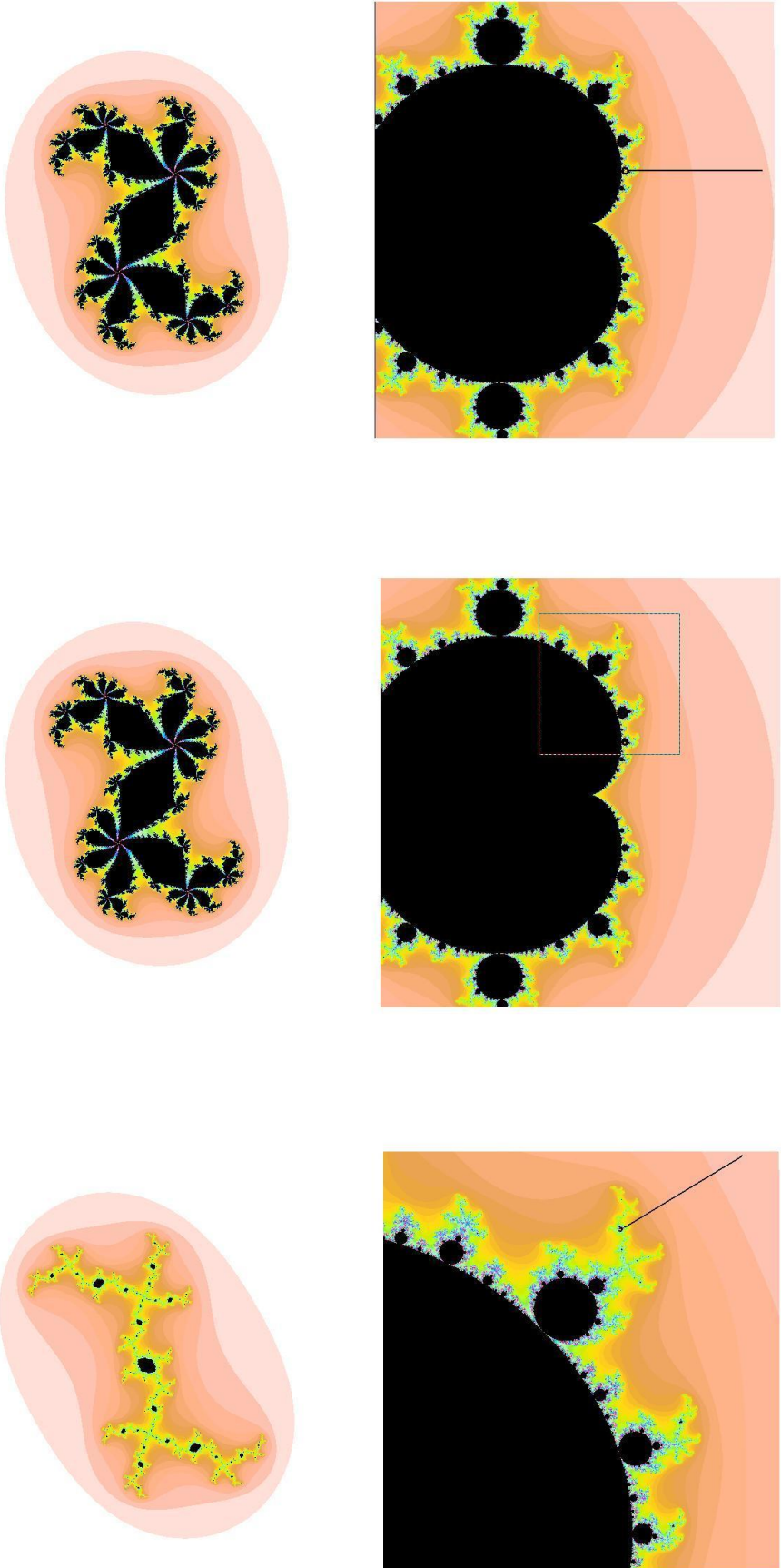

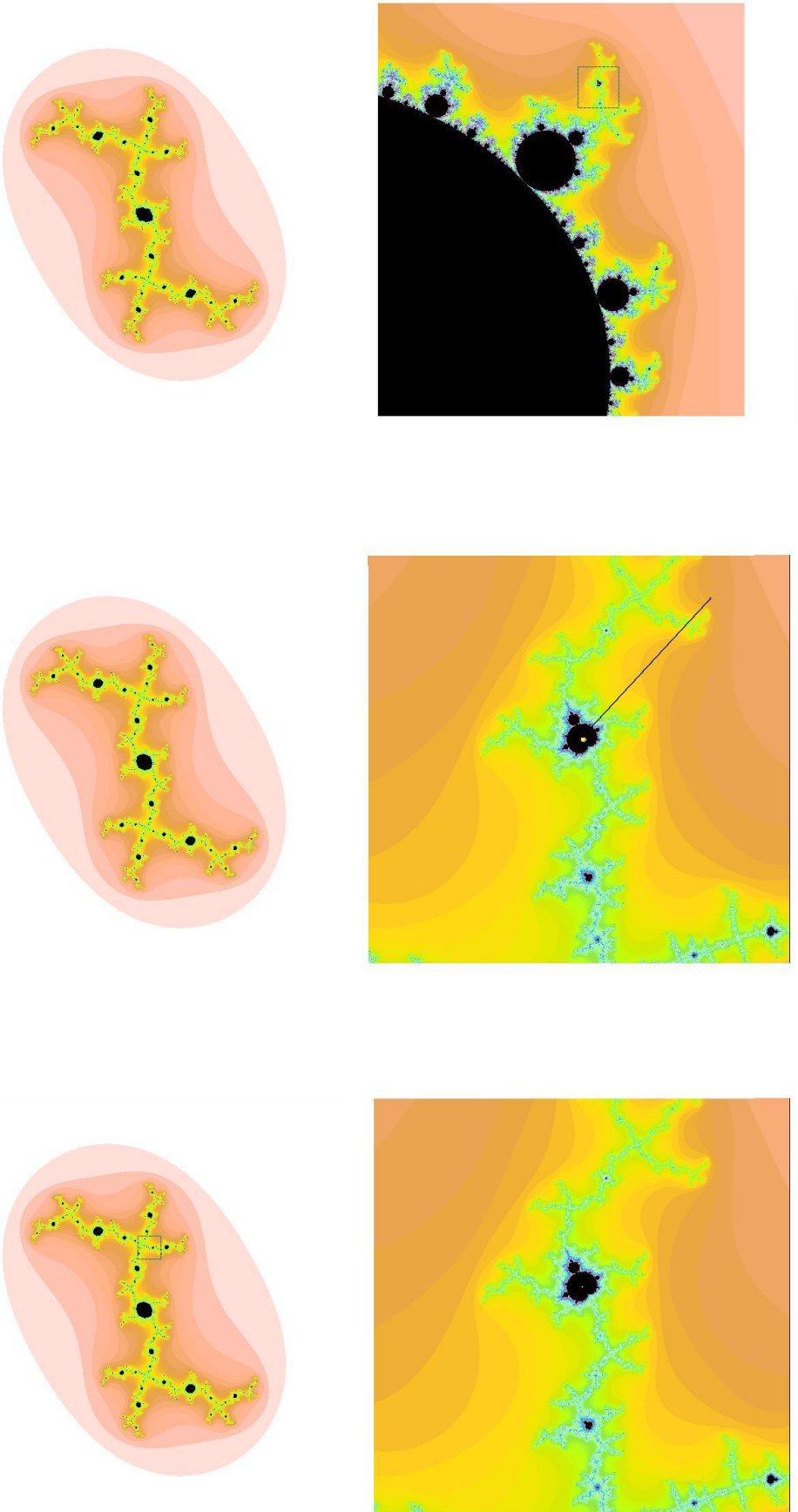

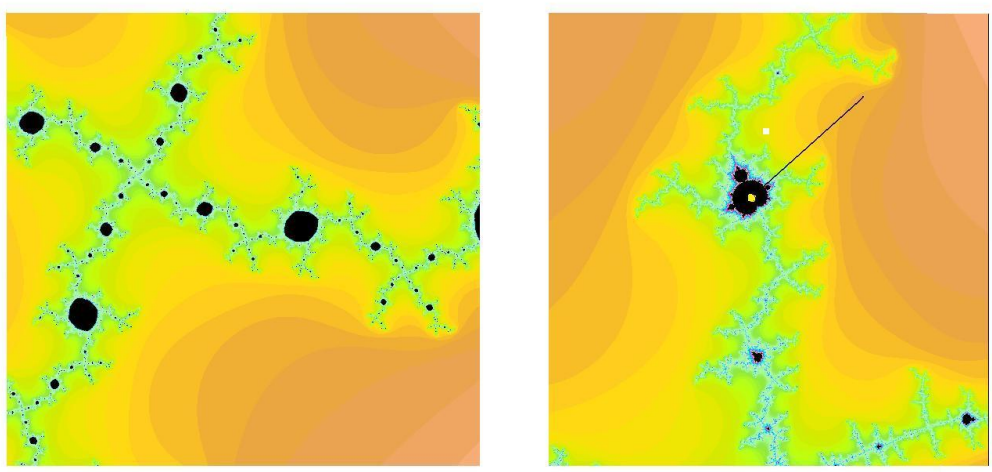



\title{
Formalismo Termodinâmico
}

\begin{abstract}
"The formalism of equilibrium statistical mechanics - which we shall call thermodynamic formalism has been developed since G. W. Gibbs to describe the properties of certain physical systems. (...) underlying the thermodynamic formalism, there are mathematical structures of great interest (...) the relation is at an abstract mathematical level, and fairly inobvious at first sight."
\end{abstract}

(DAVID RUELLE - Thermodynamic Formalism; Introduction)

Explicar a conexão existente entre Matemática e Física é uma tarefa bem complicada, para não dizer "atrevida", dada a diversidade de opiniões, presente até mesmo entre os pesquisadores destas duas áreas da Ciência. O fato, porém, é que existe uma conexão admirável entre ambas, e não há razão nenhuma para colocá-las em ambientes separados. É muito comum que as pessoas - mesmo as dotadas de preciosa capacidade intelectual - sintam a necessidade de tudo reduzir ao seu campo de idéias, insuficientes para entender toda a realidade. Na verdade, Físicos e Matemáticos (e inúmeros outros) são aliados na busca de entender a realidade, e afirmamos que esta transcende o domínio prático e material dos fatos. De fato, toda a Ciência é concebida sobre um grande todo universal, e em razão disso, também os Matemáticos prosseguem seus estudos portas a dentro da mesma realidade, em uma de suas múltiplas formas. Eis porque a formalização da Mecânica originou os conceitos do Cálculo Infinitesimal, ou porque a academia de Platão estudava geometria for its own sake. O trabalho de se entender as leis do eletromagnetismo (Teoria do Potencial) originou algumas das mais poderosas ferramentas da Análise Matemática, como Teoria da Medida, Análise Harmônica e Teoria de Distribuições. E como a realidade não tem começo nem fim, podemos nos aprofundar em qualquer direção; podemos nos ater ao campo abstrato, desenvolvendo resultados cada vez mais distantes do seu foco de origem - mas que estão ligados por um fio de idéias; ou mesmo mediar a construção do conhecimento em ambos os campos, garantindo um fluxo incessante de uma para outra parte.

As raízes do Formalismo Termodinâmico encontram-se num processo laborioso e secular de investigações sobre o estado de equilíbrio dos gases. Logo mais, apresentaremos uma pequena parte dessa teoria, encontrando-a já num grau considerável de perfeição. 


\subsection{Pressão}

Seja $T$ um endomorfismo ${ }^{1}$ contínuo de um espaço métrico compacto $X$. Chamaremos qualquer função contínua $\phi$, definida sobre $X$ e tomando valores em $\mathbf{R}$, de potencial. Nosso objetivo é definir a pressão $P(\phi)$ de qualquer potencial; $P(\phi)$ vai ser um número real estendido, dependendo apenas da topologia de $X$, da dinâmica da transformação $T$ e, obviamente, das propriedades específicas de $\phi$. Mais adiante, mostraremos que a pressão é invariante por conjugações topológicas, o que justificará o termo pressão topológica, também usado para se referir a $P(\phi)$. Quando quisermos expressar que $P(\phi)$ também depende de $T$, escreveremos $P(\phi, T)$.

A definição de $P(\phi)$ será feita em alguns passos. Primeiramente, consideramos coberturas abertas e finitas $\mathscr{U}$ de $X$, e definimos os produtos formais

$$
U_{1} U_{2} \cdots U_{n}
$$

de elementos de $\mathscr{U}$, os quais denotaremos pelas letras $U, V, \ldots$ O númeoro $n$ é chamado de tamanho do produto formal; também convencionamos que se $U$ for um produto formal de tamanho $n$, então os elementos da conbertura $\mathscr{U}$ que determinam $U$ serão denotados por $U_{1}, \ldots, U_{n}$, como acima. Distinguimos um produto formal do outro pelos abertos que compõe, pelo tamanho e pela ordem em que estes aparecem no produto. Escreveremos $x \in U$ precisamente quando $T^{i-1} x \in U_{i}$, para todo $1 \leq i \leq n$. Deste modo, $U$ possui duas interpretações, uma formal e outra conjuntística. Podemos ter dois produtos formais distintos, mas que, como conjuntos, são os mesmos. Faz-se necessária essa distinção formal; caso ela não fosse feita, por exemplo, a definição que se segue à Prop. 3.1 não seria coerente. Se $\mathscr{C}$ for uma cobertura de $X$ (isto é, a reunião de $\mathscr{C}$ é $X$ ) cujos elementos são produtos formais de mesmo tamanho $n$, escreveremos $o(\mathscr{C})=n$. Dado $m \geq 1$, definimos

$$
Z_{m}=\inf _{o(\mathscr{C})=m} \sum_{U \in \mathscr{C}} \exp \sup _{x \in U} S_{m} \phi(x)
$$

onde

$$
S_{m} \phi(x)=\phi(x)+\phi(T x) \cdots+\phi\left(T^{m-1} x\right)
$$

é a soma de Birkhoff de $\phi$ no ponto $x$, até o tempo $m$. Portanto, $Z_{m}$ depende não só de $m$, mas também de $\mathscr{U}, \phi$ e $T$. Além disso, é fácil verificar a desigualdade

$$
Z_{m} \geq \exp -m\|\phi\|_{\infty}
$$

o que nos garante que

$$
P(\phi, T, \mathscr{U}):=\inf _{m \geq 1} \frac{\log Z_{m}}{m}
$$

é um número real $>-\infty$. Nosso próximo passo será eliminar a dependência de $P(\phi, T, \mathscr{U})$ sobre $\mathscr{U}$, tomando-se o limite

\footnotetext{
${ }^{1}$ As definições dos termos não especificados podem ser encontradas no índice.
} 


$$
\lim _{|\mathscr{U}| \rightarrow 0} P(\phi, T, \mathscr{U})
$$

onde $|\mathscr{U}|$ é o diâmetro da cobertura aberta e finita $\mathscr{U}$. (Recordemos que o diâmetro de uma coleção é definido como o supremo dos diâmetros dos conjuntos que a constituem). Mas antes, exploraremos a convergência de $\left(\log Z_{m}\right) / m$.

Lema 3.1 Seja $a_{n} \in \mathbf{R}$ tal que o quociente $b_{n}=a_{n} / n$ é limitado inferiormente, com $a_{m+n} \leq a_{n}+a_{m}$. Então o limite da sequência $b_{n}$ existe e coincide com o ínfimo $c$ do seu conjunto de valores.

DEMOnSTRAÇÃo. Como $a_{n}=n a_{1}$, a sequência $b_{n}$ está no intervalo compacto $\left[c, a_{1}\right]$; $\log$, existe uma subsequência convergindo para um certo $x^{*} \in\left[c, a_{1}\right]$. Mas se fixaramos $m$ arbitrariamente, escrevendo $n=m k+j$, com $0 \leq j<m$, então veremos que

$$
\frac{a_{n}}{n} \leq \frac{a_{m k}+a_{j}}{m k} \leq \frac{a_{m}}{m}+\frac{a_{j}}{m k}
$$

de onde vem $x^{*} \leq b_{m}$, para todo $m$. Assim, $x^{*}=c$. Além disso, o mesmo argumento mostra que $c$ é o único limite subsequencial de $b_{m}$, o que termina a demonstração.

Proposição 3.1 Seja $Z_{m}$, como anteriormente. Então $Z_{m+n} \leq Z_{m} Z_{n}$; e do Lema anterior concluímos que

$$
P(\phi, T, \mathscr{U})=\lim _{m \rightarrow \infty} \frac{\log Z_{m}}{m} .
$$

Definição. Se U e $V$ forem produtos formais de uma mesma cobertura $\mathscr{U}$, com tamanhos n e m, respectivamente, então definimos

$$
U \times V=U_{1} U_{2} \cdots U_{n} V_{1} V_{2} \cdots V_{m} .
$$

DEMONSTRAÇÃo. Nas condições da definição acima, vemos que $U \times V$ é um produto de tamanho $m+n$; além disso,

$$
\exp \sup _{x \in U \times V} S_{m+n} \phi(x) \leq\left(\exp \sup _{x \in U} S_{m} \phi(x)\right)\left(\exp \sup _{y \in V} S_{n} \phi(y)\right) .
$$

O restante da demonstração segue com um argumento padrão envolvendo o ínfimo.

Agora vamos estudar o limite (3.1). Para tanto, definimos as funções

$$
a(\varepsilon)=\inf _{|\mathscr{U}| \leq \varepsilon} P(\phi, T, \mathscr{U}), \quad b(\varepsilon)=\sup _{|\mathscr{U}| \leq \varepsilon} P(\phi, T, \mathscr{U})
$$

para $\varepsilon>0$. Quando $\varepsilon \rightarrow 0$, os valores de $a(\varepsilon)$ de crescem monotonamente para o limite $a^{*}$, e $b(\varepsilon)$ decresce monotonamente para o limite $b^{*}$. Além disso, $a(\varepsilon) \leq b(\varepsilon)$. Deste modo, para se estudar o limite (3.1), devemos considerar os seguintes casos: (A) Se o 
limite de $b(\varepsilon)$ quando $\varepsilon \rightarrow 0$ for $-\infty$, então o valor de (3.1) também será $-\infty$ (veremos, $\operatorname{logo}$ adiante, que este caso não ocorre); (B) o limite de $a(\varepsilon)$ quando $\varepsilon \rightarrow 0$ é infinito e, como consequência, (3.1) também vale $\infty$; e (C) ambos os limites $a^{*}$ e $b^{*}$ são finitos e $[a(\varepsilon), b(\varepsilon)]$ é uma família descrescente cuja interseção é $\left[a^{*}, b^{*}\right]$. Neste último caso, deveremos mostrar que este intervalo limite consiste de um único ponto, o qual será, por definição, o valor do limite (3.1).

Tomemos uma cobertura aberta e finita $\mathscr{U}$ de $X$. Usando a compacidade de $X$ (número de Lebesgue), vemos que se $\delta$ for suficentemente pequeno, então qualquer cobertura aberta e finita $\mathscr{V}$ de $X$, com diâmetro menor que $\delta$, deverá satisfazer a propriedade de que para quaisquer $V \in \mathscr{V}$ e $U \in \mathscr{U}$, se $V$ interseptar $U$, então $V \subset U$. Como consequência, todo membro de $\mathscr{U}$ se escreve como reunião finita de elementos de $\mathscr{V}$. Estas propriedades se estendem de modo natural para os produtos formais. De fato, sejam $V$ e $U$ produtos formais de $\mathscr{V}$ e $\mathscr{U}$, respectivamente, ambos com mesmo tamanho $m$. Se estes produtos se interseptarem, então as interseções $V_{i} \cap U_{i}$ serão sempre não vazias e, como consequência, $V \subset U$. Temos, assim, o seguinte resultado:

( ) Sejam $\mathscr{C}_{v}$ e $\mathscr{C}_{u}$ coberturas de $X$ por meio de produtos formais de $\mathscr{V}$ e $\mathscr{U}$, respectivamente. Suponhamos que o $\left(\mathscr{C}_{v}\right)=m$ e $o\left(\mathscr{C}_{u}\right)=m$. Então todo membro de $\mathscr{C}_{v}$ está contido em algum membro de $\mathscr{C}_{u}$ e, além disso, qualquer $U$ em $\mathscr{C}_{u}$ se escreve como reunião finita de elementos de $\mathscr{C}_{v}$.

Agora vamos comparar $P(\phi, \mathscr{U})$ com $P(\phi, \mathscr{V})$. Para cada membro $U$ de $\mathscr{U}$, calculamos o supremo $d_{U}$ de todas as distâncias $|\phi(x)-\phi(y)|$, com $x, y \in U$; e depois colocamos

$$
d(\mathscr{U})=\max _{U \in \mathscr{U}} d_{U}(\phi) .
$$

Observamos que, como $\phi$ é uniformente contínua sobre o compacto $X$, à medida que o diâmetro da cobertura $\mathscr{U}$ diminui, os valores correspondentes de $d$ convergem para zero. Além disso, se escolhermos $\mathscr{C}_{u}$ econômica, no sentido de que nenhum membro de $\mathscr{C}_{u}$ se escreve como reunião de outros membros de $\mathscr{C}_{u}$, então afirmamos que existe uma função injetora $\Phi$ de $\mathscr{C}_{u}$ para $\mathscr{C}_{v}$, satisfazendo $\Phi(U) \subset U$. Para verificar essa afirmação, usamos um argumento simples de indução envolvendo $(\star)$ e o fato de $\mathscr{C}_{u}$ ser econômica. Com tudo isso que foi dito, podemos escrever

$$
\sup _{x \in U} S_{m} \phi(x) \leq \sup _{y \in V} S_{m} \phi(y)+m d ;
$$

e da injetividade de $\Phi$ segue que

$$
\sum_{U \in \mathscr{C}_{u}} \exp \sup _{x \in U} S_{m} \phi(x) \leq \mathrm{e}^{m d} \sum_{V \in \Phi\left(\mathscr{C}_{u}\right)} \exp \sup _{y \in V} S_{m} \phi(y) \leq \mathrm{e}^{m d} \sum_{V \in \mathscr{C}_{v}} \exp \sup _{y \in V} S_{m} \phi(y) .
$$

Prosseguindo com o argumento, fixamos $\mathscr{C}_{u}$ e tomamos o ínfimo sobre todas as classes $\mathscr{C}_{v}$ nas condições acima; em seguida tomamos o ínfimo sobre $o\left(\mathscr{C}_{u}\right)=m$ e concluímos daí que

$$
P(\phi, \mathscr{U}) \leq P(\phi, \mathscr{V})+d(\mathscr{U})
$$


A primeira informação que extraímos de (3.2) é que $a(\varepsilon)$ é limitada inferiormente; logo, o caso (A) está excluído. O caso (B) já foi previamente analisado; a conclusão é que o limite (3.1) existe e vale $\infty$. Examinando o caso (C) - tendo em vista (3.2) -, vemos que

$$
P(\phi, \mathscr{U}) \leq a^{*}+d(\mathscr{U}) .
$$

Mas $d(\mathscr{U})$ tende a zero quando $|\mathscr{U}| \rightarrow 0 ; \operatorname{logo}, b^{*} \leq a^{*}$, como queríamos.

Resumimos o que discutimos na seguinte

Proposição 3.2 (Definição de pressão) $O$ limite (3.1) existe e pertence à $(-\infty, \infty]$. Denotaremos tal limite por $P(\phi)$; ele é chamado de pressão topológica do potencial $\phi$, ou, simplesmente, pressão de $\phi$.

Diremos que dois endomorfismos contínuos $(T, X)$ e $(S, Y)$ estão topologicamente conjugados se existir um homeomorfismo $\pi$ de $X$ para $Y$ satisfazendo

$$
S \pi(x)=\pi T(x)
$$

para todo $x \in X$. Conjugações topológicas servem para distinguir diferentes tipos de dinâmica sob o ponto de vista espacial. Uma vez estabelecida a conjugação $\pi$, à cada potencial $\phi$ de $S$ corresponde um potencial $\psi=\phi \circ \pi$ de $T$. É natural esperar que

Proposição 3.3 De acordo com as hipóteses acima, $P(\psi, T)=P(\phi, S)$.

DEMOnStRAÇÃo. Seja $\mathscr{U}$ uma cobertura aberta e finita de $Y$. Então $\mathscr{V}^{2}=\pi^{-1} \mathscr{U}$ é uma cobertura aberta e finita de $X$, com

$$
P(\phi, S, \mathscr{U})=P(\psi, T, \mathscr{V}),
$$

o que pode ser verificado com a definição de $P(\phi, T, \mathscr{U})$ e identidade que define conjugação. Nosso objetivo é mostrar que $P(\phi, S)$ é menor ou igual a $P(\psi, T)$ e, para tanto, podemos assumir que a pressão de $\psi$ é finita. Isso nos permite usar (3.2), com $\psi$ no lugar de $\phi$, e $d_{\psi}(\mathscr{V})$ em vez de $d(\mathscr{U})$. Juntamente com (3.3), vem

$$
P(\phi, S, \mathscr{U}) \leq P(\psi, T)+d_{\psi}(\mathscr{V}) .
$$

Agora observamos que o diâmetro de $\mathscr{V}$ converge para zero quando $|\mathscr{U}| \rightarrow 0$; logo, $P(\phi, S) \leq P(\psi, T)$. Obviamente, podemos trocar os papéis de $\psi$ e $\phi$ nessa última desigualdade, obtendo o resultado desejado.

Ainda voltaremos a estudar outras propriedades da pressão. Um dos nossos objetivos será estender sua definição para potenciais complexos.

\footnotetext{
${ }^{2}$ A pré-imagem $f^{-1} \mathscr{C}$ de uma classe de conjuntos $\mathscr{C}$ é $\left\{f^{-1} C ; C \in \mathscr{C}\right\}$.
} 


\title{
3.2 Entropia
}

\begin{abstract}
“( $(\cdots)$ I thought of calling it information. But the word was overly used, so I decided to call it uncertainty. When I discussed it with John Von Neumann, he had a better idea. He told me, You should call it entropy, for two reasons. In first place your uncertainty has been used in statistical mechanics under that name, so it already has a name. In second, and more important, no one knows what entropy really is, so in a debate you will always have the advantage."
\end{abstract}

(Claude Shannon - Citação feita em [32])

O conceito de entropia está ligado ao nível de complexidade de um sistema. Sua formulação original basea-se em observações empíricas e sofreu algumas adaptações em abordagens mais formais, notadamente em Teoria da Informação. A formulação que apresentaremos é devida à Kolmogorov e Sinai (1958/1959). Visamos apenas definir de modo formal este conceito. O campo, todavia, é bem vasto, e o leitor interessado num estudo pormenorizado do assunto (com motivações realistas) pode consultar a bibliografia

\section{Referências: [50] [37] [49] [28] [6] [8] [32].}

Nosso objetivo é definir a entropia $h_{\mu}(T)$ de uma transformação $T$ de uma espaço de probabilidade $(X, \mathscr{B}, \mu)$. Vamos supor que $T$ preserva $\mu$, i.é., que $\mu T^{-1}(B)=\mu(B)$, para todo $B \in \mathscr{B}$. Começaremos definindo entropia de partições finitas. Recordemos que uma partição de $X$ é uma classe disjunta de subconjuntos cuja reunião é todo o $X$. Denotaremos partições com as letras gregas $\xi, \eta, \ldots$; cada elemento de uma partição é também chamado de átomo. Vamos considerar apenas partições formadas por um número finito de átomos, os quais deverão ser subconjuntos mensuráveis do espaço de probabilidade. Se os átomos de $\xi$ forem $A_{1}, \ldots, A_{n}$, então definimos a entropia $h_{\mu}(\xi)$ de $\xi$ com respeito a $\mu$ por

$$
h_{\mu}(\xi)=-\sum_{i=1}^{n} \mu\left(A_{i}\right) \log \mu\left(A_{i}\right)
$$

onde $\log$ é o logaritmo natural e $0 \log 0=0$. Notemos que $h_{\mu}(\xi) \geq 0$.

Podemos pensar nos átomos $A_{i}$ da partição $\xi$ como os resultados possíveis de um experimento, cada um ocorrendo com probabilidade $\mu\left(A_{i}\right)$. A entropia de $\xi$ foi formulada de modo a medir o grau de incerteza deste experimento. Mais detalhes podem ser encontrados na bibliografia (por exemplo, [49], pág. 77).

Dadas duas partições $\xi$ e $\eta$, definimos o seu refinamento comum $\xi \vee \eta$ como sendo a partição cujos átomos são todos da forma $A \cap C$, onde $A$ é um átomo de $\xi$ e $C$ é um átomo de $\eta$. A entropia condicional de $\xi$ dado $\eta$ é o número

$$
h_{\mu}(\xi \backslash \eta)=h_{\mu}(\xi \vee \eta)-h_{\mu}(\eta) \text {. }
$$

Pode-se verificar facilmente que se $A_{1}, \ldots, A_{k}$ forem os átomos de $\xi$ e $C_{1}, \ldots, C_{\ell}$ os átomos de $\eta$, então 


$$
h_{\mu}(\xi \backslash \eta)=-\sum_{j=1}^{\ell} \mu\left(C_{j}\right) \sum_{i=1}^{k} \mu\left(A_{i} \backslash C_{j}\right) \log \mu\left(A_{i} \backslash C_{j}\right)
$$

onde

$$
\mu\left(A \backslash C_{j}\right)=\frac{\mu\left(A \cap C_{j}\right)}{\mu\left(C_{j}\right)}
$$

é a medida condicinal de $\mu$ em $C_{j}$ (não confundir " " com o sinal de diferença de conjuntos).

Observemos que a entropia de uma partição $\xi$ não depende dos seus átomos, mas especificamente do vetor ${ }^{3}$ de probabilidades $\left(\mu A_{i}\right)_{i}$ que lhe está associado. Uma das consequências desse fato é que

$$
h_{\mu}\left(T^{-1} \xi\right)=h_{\mu}(\xi)
$$

pois $T$ preserva $\mu$. A fórmula (3.4) nos fornece uma interpretação mais intuitiva da entropia condicional. Naturalmente, $\xi$ induz uma partição $\xi_{j}$ de cada átomo $C_{j}$ de $\eta \mathrm{e}$, além disso, $\mu_{j}=\mu\left(\cdot \backslash C_{j}\right)$ é uma probabilidade de $C_{j}$. A equação (3.4) nos diz que a entropia de $\xi$ dado $\eta$ é simplesmente a média ponderada das entropias $h_{\mu_{j}}\left(\xi_{j}\right)$.

Lema 3.2 A função $\phi(x)$, dada por $\phi(0)=0$ e $\phi(x)=x \log x$ para $x>0$, é convexa, ou seja,

$$
\phi\left(\alpha_{1} x_{1}+\cdots+\alpha_{n} x_{n}\right) \leq \alpha_{1} \phi\left(x_{1}\right)+\cdots+\alpha_{n} \phi\left(x_{n}\right),
$$

para todo vetor de probabilidades $\left(\alpha_{i}\right)$, com $x_{i} \geq 0$.

Demonstração. Este é um resultado de Análise introdutória. Basta calcular a segunda derivada de $\phi$ e ver que ela é estritamente positiva sobre $(0, \infty)$.

Agora invertemos a ordem dos somatórios em (3.4), de modo a contarmos primeiro $i$ e depois $j$; fazendo $\alpha_{j}=\mu\left(C_{j}\right)$ e $x_{j}=\mu\left(A_{i} \backslash C_{j}\right)$, do Lema anterior concluímos que

$$
h_{\mu}(\xi \backslash \eta) \leq h_{\mu}(\xi)
$$

e como consequência,

$$
h_{\mu}(\xi \vee \eta) \leq h_{\mu}(\xi)+h_{\mu}(\eta) .
$$

De agora em diante, se $\xi_{1}, \ldots, \xi_{n}$ forem partições finitas, então denotaremos

$$
\bigvee_{i=1}^{n} \xi_{i}=\xi_{1} \vee \xi_{2} \vee \cdots \vee \xi_{n}
$$

Para introduzirmos a dinâmica da transformação $T$ na definição de entropia, primeiramente consideramos a sequência

\footnotetext{
${ }^{3} \mathrm{Um}$ vetor de probabilidades é toda $n$-upla de números reais $\alpha_{i} \geq 0$ cuja soma $\alpha_{1}+\cdots+\alpha_{n}$ é 1 .
} 


$$
a_{n}=h_{\mu}\left(\bigvee_{i=0}^{n-1} T^{-i} \xi\right)
$$

Como

$$
\begin{aligned}
a_{n+p} & =h_{\mu}\left(\left(\bigvee_{i=0}^{p-1} T^{-i} \xi\right) \vee T^{-p} \bigvee_{i=0}^{n-1} T^{-i} \xi\right) \\
& \leq h_{\mu}\left(\bigvee_{i=0}^{p-1} T^{-i} \xi\right)+h_{\mu}\left(\bigvee_{i=0}^{n-1} T^{-i} \xi\right) \\
& =a_{p}+a_{n},
\end{aligned}
$$

do Lema 3.1 concluímos que $a_{n} / n$ converge para um número real $\geq 0$, que denotaremos $h_{\mu}(T, \xi)$. Como última etapa, definimos a entropia $h_{\mu}(T)$ da transformação $T$ como sendo o supremo de $h_{\mu}(T, \xi)$ tomado sobre todas as partições finitas $\xi$. Observamos que o valor da entropia pode muito bem ser infinito.

A entropia mede o grau de complexidade do sistema $T$, e sua importância reside no fato de que ela é um invariante métrico do sistema. De uma forma bem sucedida, ela distingue alguns exemplos importantes de dinâmica. Aliás, foi neste contexto que Kolmogorov introduziu o conceito entropia em 1958. A versão que apresentamos é o resultado de uma pequena modificação feita por Sinai em 1959.

Colocaremos de forma precisa a observação que fizemos acima. Consideremos duas transformações de espaços de probabiblidade

$$
T_{1}:\left(X_{1}, \mathscr{B}_{1}, \mu_{1}\right) \hookleftarrow, T_{2}:\left(X_{2}, \mathscr{B}_{2}, \mu_{2}\right) \hookleftarrow .
$$

Sejam $F_{1}$ de $F_{2}$ subconjuntos de medida total ${ }^{4}$ de $X_{1}$ e $X_{2}$, respectivamente. Uma bijeção mensurável $\phi$ entre conjuntos de medida total $F_{1}$ e $F_{2}$ de $X_{1}$ e $X_{2}$, respectivamente, será um isomorfismo do par $\left(T_{1}, T_{2}\right)$ quando (i) ambos os conjuntos de medida total forem invariantes $\left(T_{i} F_{i} \subset F_{i}\right)$; (ii) $\mu_{1} \phi^{-1}(B)=\mu_{2}(B)$, para todo subconjunto mensurável $B$ de $F_{2}$ (ou seja, $\phi$ preserva as medidas induzidas sobre os conjuntos invariantes); e (iii) quando $\phi$ for uma conjugação entre as restrições $\left.T_{i}\right|_{F_{i}}$ :

$$
T_{2} \phi(x)=\phi T_{1}(x)
$$

para todo $x \in F_{1}$.

Um modo alternativo de se lidar com conjugações que estão definidas apenas num conjunto de medida total é abstrair a estrutura do espaço de medida por uma estrutura essencialmente algébrica. Do ponto de vista matemático - i.é., para se usar em argumentações -, essa é a abordagem mais natural.

Definimos uma relação de equivalência na $\sigma$-álgebra $\mathscr{B}$, declarando que dois conjuntos $A$ e $B$ estão relacionados se, e somente se, a medida da diferença simétrica $A \triangle B$

\footnotetext{
${ }^{4} B$ tem medida total quando a medida de seu complementar é nula.
} 
for nula. Denotamos a partição obtida a partir dessa relação por $\mathscr{B}^{*}$; indicaremos seus elementos com as letras $x, y, z, \ldots$ Se $A$ for um representante de $x$, então escreveremos $x=A^{*}$. Existem duas operações binárias bem interessantes em $\mathscr{B}^{*}$. Definimos $x+y$ como sendo a classe de equivalência da reunião $A \cup B$, onde $A$ e $B$ são quaisquer representantes de $x$ e $y$, respect. Deve-se verificar que essa definição independe da escolha de $A$ e $B$. Usando os mesmos argumentos, definimos

$$
x y=(A \cap B)^{*} .
$$

Usaremos o símbolo 1 para indicar $X^{*}$ e $0=\emptyset^{*}$. Verifica-se que

(i) $x(y+z)=x y+x z$;

(ii) $x+y=y+x ; x y=y x$;

(iii) $0+x=x ; 1+x=1$;

(iv) $1 x=x ; 0 x=0$.

Diremos que $y$ é um complemento de $x$ - denotando $y=x^{c}$ - quando tivermos $x y=$ 0 e $x+y=1$. Pelas propriedades acima, se $x$ tiver um complemento, então ele será único. É fácil ver que, pelo modo como as operações,$+ \cdot$ foram definidas, todo $x \mathrm{em}$ $\mathscr{B}^{*}$ possui um complementar em $\mathscr{B}^{*}$. Podemos definir uma "medida" $\mu^{*}$ na álgebra medida colocando

$$
\mu^{*}(x)=\mu(A),
$$

se $x=A^{*}$. Usando um argumento padrão, vemos que $\mu^{*}$ está bem definida e satisfaz: (i) $\mu^{*}(0)=0$; (ii) $\mu^{*}(x)>0$ se $x \neq 0$. Somas e produtos infinitos são definidos em $\mathscr{B}^{*}$ da seguinte forma:

$$
\begin{gathered}
\sum_{j=1}^{\infty} x_{j}=x_{1}+x_{2}+x_{3}+\cdots:=\left(\bigcup_{j=1}^{\infty} A_{j}\right)^{*} ; \\
\prod_{j=1}^{\infty} x_{j}=x_{1} x_{2} \cdots:=\left(\bigcap_{j=1}^{\infty} A_{j}\right)^{*},
\end{gathered}
$$

onde $x_{j}=A_{j}^{*}$. É possível verificar que se $x_{i} x_{j}=0$ quando $i \neq j$, então

$$
\mu^{*}\left(x_{1}+x_{2}+\cdots\right)=\mu^{*}\left(x_{1}\right)+\mu^{*}\left(x_{2}\right)+\cdots
$$

O conjunto $\mathscr{B}^{*}$, juntamente com $+, \cdot, \mu^{*}$, é chamado de álgebra medida de $(\mathscr{B}, \mu)$.

Definição. Duas álgebras medidas $\left(\mathscr{B}_{1}^{*}, \mu_{1}^{*}\right),\left(\mathscr{B}_{2}^{*}, \mu_{2}^{*}\right)$ serão isomorfas quando existir uma bijeção $\Phi$ de $\mathscr{B}_{1}^{*}$ para $\mathscr{B}_{2}^{*}$ satisfazendo

(i) $\mu_{2}^{*}(\Phi x)=\mu_{1}^{*}(x)$;

(ii) $\Phi(x+y)=\Phi(x)+\Phi(y)$;

(iii) $\Phi(x y)=\Phi(x) \Phi(y)$;

(iv) $\Phi(x)^{c}=\Phi\left(x^{c}\right)$;

(v) $\Phi\left(x_{1}+x_{2}+\cdots\right)=\Phi\left(x_{1}\right)+\Phi\left(x_{2}\right)+\cdots$; 
(vi) $\Phi\left(x_{1} x_{2} \cdots\right)=\Phi\left(x_{1}\right) \Phi\left(x_{2}\right) \cdots$.

Naturalmente, algumas das propriedades acima decorrem umas das outras. Além disso, podemos ver que

$$
\Phi\left(A^{*}\right)=B^{*} \Longrightarrow \mu_{1}(A)=\mu_{2}(B) .
$$

Voltemos ao nosso sistema dinâmico $T$ (ver início da seção). Existe uma aplicação natural $T^{*}$ de $\mathscr{B}^{*}$ em $\mathscr{B}^{*}$, dada por $T^{*} x=\left(T^{-1} A\right)^{*}$, se $x=A^{*}$. Como $T$ preserva $\mu$, essa função está bem definida; além disso

$$
\left(T^{*}\right)^{n} A^{*}=\left(T^{-n} A\right)^{*} \text { e } T^{*}\left(x^{c}\right)=\left(T^{*} x\right)^{c} .
$$

Verifica-se ainda que:

$$
T^{*}\left(\sum_{j=1}^{\infty} x_{j}\right)=\sum_{j=1}^{\infty} T^{*}\left(x_{j}\right) \text { e } T^{*}\left(\prod_{j=1}^{\infty} x_{j}\right)=\prod_{j=1}^{\infty} T^{*}\left(x_{j}\right) .
$$

Definição. Suponhamos que as duas transformações $T_{1}, T_{2}$ em (3.9) preservem as medidas $\mu_{1}, \mu_{2}$, respectivamente. Diremos que $T_{1}$ é conjugada à $T_{2}$ quando existir um isomorfismo $\Phi$ entre as álgebras medidas $\mathscr{B}_{1}^{*}$ e $\mathscr{B}_{2}^{*}$ satisfazendo

$$
T_{2}^{*} \circ \Phi=\Phi \circ T_{1}^{*}
$$

Todo isomorfismo do par $\left(T_{1}, T_{2}\right)$ induz de modo natural uma conjugação $\Phi$ entre as respectivas álgebras medidas. De fato, se $\phi$ for o isomorfismo de $\left(T_{1}, T_{2}\right)$, definido entre conjuntos de medida total $F_{1}$ e $F_{2}$, então basta definir

$$
\Psi(x)=\left[\phi^{-1}\left(A \cap F_{2}\right)\right]^{*},
$$

onde $x=A^{*}$, e depois tomar $\Phi=\Psi^{-1}$.

Teorema 3.1 Suponhamos que os dois sistemas $T_{1}$ e $T_{2}$ em (3.9) preservem suas medidas, e que eles sejam conjugados por um isomorfismo $\Phi$ das álgebras medidas $\mathscr{B}_{1}^{*}$ e $\mathscr{B}_{2}^{*}$. Então

$$
h_{\mu_{1}}\left(T_{1}\right)=h_{\mu_{2}}\left(T_{2}\right) \text {. }
$$

DEMONSTRAÇÃo. Para toda partição $\xi$ de $X_{1}$, construiremos uma outra partição $\eta$ de $X_{2}$, com

$$
h_{\mu}\left(T_{1}, \xi\right)=h_{\mu}\left(T_{2}, \eta\right)
$$

E tomando-se o supremo sobre todas as possíveis $\xi$, vemos que a entropia de $T_{1}$ é menor ou igual que a entropia de $T_{2}$. $\mathrm{O}$ argumento pode ser usado na direção inversa, concluindo que as entropias são realmente iguais. Deste modo, precisamos apenas desenvolver um meio de se construir partições $\eta$ nas condições acima.

Sejam $A_{1}, \ldots, A_{r}$ os átomos da partição $\xi$. Então os elementos correspondentes $x_{j}=A_{j}^{*}$ satisfazem 


$$
\sum_{j=1}^{r} x_{j}=1 \text { e } x_{i} x_{j}=0
$$

e como $\Phi$ é um isomorfismo, as imagens $y_{j}=\Phi\left(x_{j}\right)$ gozam das mesmas propriedades. Encontramos, assim, uma partição $\eta$ de $X_{2}$ cujos átomos $B_{j}$ satisfazem $y_{j}=B_{j}^{*}$. Falta mostrar que as entropias dessas duas partições são as mesmas. Para tanto, é suficiente estabelecer uma correspondência biunívoca entre os átomos das partições

$$
\bigvee_{j=0}^{n-1} T^{-j} \xi, \bigvee_{j=0}^{n-1} T^{-j} \eta
$$

preservando-se as medidas dos mesmos. Recordemos que a entropia de uma partição não depende da disposição dos átomos, tampouco do espaço em si, mas apenas do vetor de probabilidades associado à partição. Todo átomo $A$ da primeira partição é uma interseção de $n$ pré-imagens

$$
A=\bigcap_{j=0}^{n-1} T_{1}^{-j} A_{i_{j}} .
$$

Seja $B$ a interseção das pré-imagens correspondentes $T_{2}^{-j} B_{i_{j}}$. Notemos que $B$ é unicamente determinado a partir de $A$, e que a correspondência $A \mapsto B$ é bijetora.

Como

$$
\begin{aligned}
\Phi\left(A^{*}\right) & =\Phi\left(\prod_{j=0}^{n-1}\left(T_{1}^{*}\right)^{j} A_{i_{j}}^{*}\right) \\
& =\prod_{j=0}^{n-1} \Phi\left(T_{1}^{*}\right)^{j} A_{i_{j}}^{*}=\prod_{j=0}^{n-1}\left(T_{2}^{*}\right)^{j} \Phi A_{i_{j}}^{*} \\
& =\prod_{j=0}^{n-1}\left(T_{2}^{*}\right)^{j} B_{i_{j}}^{*}=\prod_{j=0}^{n-1}\left(T_{2}^{-j} B_{i_{j}}\right)^{*} \\
& =B^{*}
\end{aligned}
$$

segue-se que $A$ e $B$ possuem a mesma medida, o que termina a demonstração.

\subsection{Transformações expansoras e estados de equilíbrio}

Nas duas seções precedentes, definimos os conceitos de pressão e entropia, os quais estão relacionados pelo

Teorema 3.2 (Princípio Variacional) Seja T um endomorfismo contínuo de um espaço métrico compacto $Y$. Então para todo potencial real $\phi \in C(Y)$, 


$$
P(\phi, T)=\sup _{\mu \in M_{T}(Y)}\left(h_{\mu}(T)+\int \phi d \mu\right),
$$

onde $M_{T}(Y)$ é o conjunto de todas as medidas de probabilidade invariantes ${ }^{5}$ por $T$, definidas na $\sigma$-álgebra de Borel de $Y$.

DEMONSTRAÇÃo. Ver [6], pág. 40.

Uma medida $\mu \in M_{T}(Y)$ será chamada de estado de equilíbrio para $\phi$ (com respeito à $T$ ) quando o supremo acima for atingido em $\mu$, i.é., a soma da entropia de $T$ com respeito à $\mu$ juntamente com a média do potencial (com respeito a $\mu$ ) for igual à pressão deste.

Neste tópico, estudaremos transformações expansoras, objetivando relacionar estes dois conceitos com propriedades espectrais de um operador agindo sobre $C(Y)$, o qual nos fornece importantes propriedades sobre o sistema.

\subsubsection{Transformações Expansoras}

Um endomorfismo $f$ de um espaço métrico compacto $X$ será expansor quando existir uma constante $\mu>1$ tal que (i) $f$ é um homemorfismo local e (ii) todo ponto $p$ de $X$ possuir uma vizinhança $V(p)$ tal que $f^{-1} V$ é uma reunião disjunta e finita de abertos $U_{1}, \ldots, U_{m_{p}}$, sendo cada restrição $f \mid U_{i}$ um homeomorfismo sobre $^{6} V$ satisfazendo à desigualdade

$$
d(f x, f y) \geq \mu d(x, y)
$$

sobre cada $U_{i}$. De (i) segue que $f$ é localmente injetora e, usando a compacidade de $X$ (número de Lebesgue) encontramos um $c>0$ tal que $f$ é injetora sobre qualquer conjunto de diâmetro $<c$.

A definição de transformação expansora que apresentamos é uma versão equivalente da que comumente se encontra na literatura (ver [13], por exemplo). Usaremos a condição (ii) para definir o operador de Ruelle-Perron-Frobenius.

Muitos exemplos de transformações expansoras surgem do seguinte contexto. Seja $M$ uma variedade Riemanniana compacta e sem bordo. Denotaremos a norma provinda da métrica no plano tangente $T_{x} M$ por $\|\cdot\|_{x}$, ou, simplesmente, $\|\cdot\|$, o que não será motivo de confusão. Seja $U$ um subconjunto aberto e não vazio de $M$, no qual a distância Riemanniana $d$ está definida, e consideremos uma aplicação $C^{1}$-diferenciável $f$ de $U$ em $M$, tal que $f^{-1} U \subset U$, com um $\mu>1$ satisfazendo

$$
\|D f(x) \cdot v\| \geq \mu\|v\|,
$$

para todo $x \in U$, onde a constante $\mu$ independe de $x$. A sequência de abertos $U_{n}=f^{-n} U$ é encaixada e decrescente. Vamos supor que sua interseção $\Lambda$ é um compacto não vazio. Como $f^{-1} \Lambda=\Lambda$, em particular concluímos que $\Lambda$ é invariante por $f$ e, portanto, $(f, \Lambda)$ é um sistema dinâmico. Afirmamos que

\footnotetext{
${ }^{5} \mu\left(T^{-1} A\right)=\mu(A)$, para todo mensurável $A$.

${ }^{6}$ A palavra sobre é utilizada para indicar que $f$ é sobrejetora.
} 
Proposição $3.4(f, \Lambda)$ é expansor.

DemonstraçÃo. Primeiramente observamos que, pelo Teorema da Função inversa (ver, por exemplo, [30]), $f$ é um difeomorfismo local de $U_{1}$ sobre $U$. Assim, dados $x \in U$ e $a \in f^{-1} x$, existem vizinhanças $A(a)$ e $B(x)$ tais que $f$ é um difeomorfismo de $A$ sobre $B$. Podemos escolher $B(x)$ de modo que quaisquer dois pontos de $B(x)$ possam ser ligados por uma geodésica minimizante ainda contida em $B$ (ver [10], pág. 81). Dados $z$ e $w$ em $A$, seja $\beta \subset B$ uma geodésica definida no intervalo [0,1], ligando $f z$ a $f w$, cujo comprimento $\ell(\beta)$ vale $d(f z, f w)$. Então

$$
d(z, w) \leq \ell\left(f^{-1} \beta\right)=\int_{0}^{1}\left\|D f^{-1}(\beta(t)) \beta^{\prime}(t)\right\| d t \leq \frac{1}{\mu} d(f z, f w) .
$$

Agora nos restringimos ao caso em que $x$ está em $\Lambda$. Consequentemente, $a$ também está em $\Lambda$ e, do que vimos, segue-se que $f$ é um homeomorfismo local de $A \cap \Lambda$ sobre $B \cap \Lambda$, satisfazendo a condição (3.13) em todo o aberto relativo $A \cap \Lambda$. De agora em diante, vamos procurar adequar este resultado de modo a concluir (ii) da definição de transformação expansora. Toda noção topológica será a do espaço métrico $(\Lambda, d)$, onde $d$ é a distância Riemanniana definida sobre $U$. Como $f$ é localmente injetora, toda préimagem $f^{-1} x$ consiste de um número finito de pontos $a_{1}, \ldots, a_{m}$, onde $m$, obviamente, depende de $x$. Para cada $i$ variando de 1 até $m$, existem vizinhanças $A_{i}\left(a_{i}\right), B_{i}(x)$ tais que $f$ é um homemorfismo de $A_{i}$ sobre $B_{i}(x)$, como acima. Definimos

$$
\varphi_{x, i}=\left.f^{-1}\right|_{B_{i}(x)}
$$

Considerando-se a interseção de todos os $B_{i}$, vemos que, na definição acima, o domínio de $\varphi_{x, i}$ independe de $i$. Em seguida usamos a compacidade de $\Lambda$ : existe um $r_{0}$ suficientemente pequeno tal que podemos escolher $B_{i}(x)=B_{r_{0}}(x)$ (bola aberta centrada em $x$ e de raio $r_{0}$ ). Para completar a demonstração, devemos mostrar que podemos escolher $r_{0}$ ainda menor, de modo que, independentemente de $x$, os conjuntos $\varphi_{x, i} B_{r_{0}}(x)$ sejam dois a dois disjuntos, com

$$
f^{-1} B_{r_{0}}(x)=\bigcup_{i=1}^{m} \varphi_{x, i} B_{r_{0}}(x) .
$$

O argumento que usaremos é elementar, mas um tanto técnico. Recordemos que $f$ é localmente injetora e que $\Lambda$ é compacto. Existe uma constante $c>0$ tal que os pontos $a_{i}$ da pré-imagem são igualmente espaçados, ou seja, ${ }^{7}$

$$
d\left(a_{i}, a_{j}\right) \geq c \delta_{i j}
$$

Além disso, cada $\varphi_{x, i} B_{r_{0}}(x)$ está contido numa bola de raio menor que $r_{0} / \mu$, centrada em $a_{i}$. É claro que, independentemente de $x$, podemos tomar $r_{0}$ de modo que, para cada $x$ fixo, os conjuntos $\varphi_{x, i} B_{r_{0}}(x)$ sejam dois a dois disjuntos. Se $d(x, y)<r_{0}$, então

\footnotetext{
${ }^{7} \delta_{i j}$ vale 1 se $i \neq j$ e 0 caso contrário.
} 
afirmamos que toda pré-imagem $b \in f^{-1} y$ está na reunião destes conjuntos. Isso é o que falta para terminar a demonstração. Por contradição, suponhamos que exista um ponto $b$ da pré-imagem de $y$ que esteja fora da reunião dos conjuntos $\varphi_{x, i} B_{r_{0}}(x)$. Então $y$ possui pelo menos $m+1$ pré-imagens $b_{j}$, as quais, por sua vez, determinam $m+1$ funções

$$
\varphi_{y, j}: B_{r_{0}}(y) \rightarrow \Lambda,
$$

cujas imagens são duas a duas disjuntas. Como $x$ está no domínio destas funções, a conclusão é que $x$, também, possui $m+1$ pré-imagens, o que é uma contradição.

Proposição 3.5 Seja J o conjunto de Julia de uma função racional hiperbólica $f$. Então $(f, J)$ é expansor.

Demonstração. Seja $W$ o aberto contedo $J$ sobre o qual está definida a métrica conforme; seja $\rho$ a distância Riemanniana provinda dessa métrica - que, a rigor, está definida apenas localmente, mas para provar que $f$ é expansora, interessam apenas as distâncias de pontos "próximos". Usando-se argumentos parecidos com o do item anterior, pode-se provar que todo ponto $z$ de $J$ possui uma vizinhança $V$, com $f V \subset W$, tal que

$$
\rho(f a, f b) \geq \mu \rho(a, b)
$$

sempre que $a, b \in V$.

Sabemos que $J$ é completamente invariante, e que $f$ não tem pontos críticos sobre $J$. Com isso, vemos que $f$ é um difeomorfismo local em cada ponto de $J$; de onde concluímos que $f$ é localmente injetora sobre $J$. Como $J$ é compacto, isso faz com que todo ponto $w \in J$ possua um número finito de pré-imagens $z_{1}, \ldots, z_{m}$ (com $m$ dependendo de cada ponto), todas contidas em $J$. Escolhemos uma vizinhança $W_{1} \subset W$ de $J$, com a propriedade $f^{-1}\left(W_{1}\right) \subset W$. (Observemos que $f W^{c}$ é compacto e está contido no complementar de $J$, que é aberto; basta escolher $W_{1}$ não interseptando este compacto). Usando a compacidade de $J$, vemos que existe um $\delta>0$ tal que toda bola $B_{\delta}(w)$ centrada em pontos $w$ de $J$ ainda está contida em $W_{1}$, com a propriedade adicional de que se as pré-imagens de $w$ forem $z_{1}, \ldots, z_{m}, m=m(w)$, então teremos difeomorfismos

$$
f: V_{i}\left(z_{i}\right) \rightarrow B_{\delta}(w)
$$

$\operatorname{com} V_{i} \subset W \mathrm{e}$

$$
\rho(f a, f b) \geq \mu \rho(a, b)
$$

sobre cada $V_{i}$. Uma análise envolvendo esta última desigualdade e o fato de $f$ ser localmente injetora nos diz que podemos tomar $\delta$ suficientemente pequeno, de modo que, independentemente de $w$, a reunião dos $V_{i}$ seja disjunta e dê toda a pré-imagem $f^{-1} B_{\delta}(w)$. Quando restringimos estes difeomorfismos à $J$ obtemos que $f$ é expansora.

Pode-se mostrar que para toda transformação expansora $f$ existe um $\varepsilon>0$ - também chamado de constante de expansividade - tal que, para quaisquer pontos $x$ e $y$,

$$
d\left(f^{k} x, f^{k} y\right) \leq \varepsilon, \forall k, \Longrightarrow x=y \text {. }
$$


Proposição 3.6 Seja $\mu$ uma medida de probabilidade invariante pela dinâmica da transformação expansora $f$, com constante de expansividade $\varepsilon$. Se $\xi$ for uma partição de diâmetro menor que $\varepsilon$, então

$$
h_{\mu}(\xi, f)=h_{\mu}(f) \text {. }
$$

DEMONSTRAÇÃo. Ver [6], pág. 32.

\subsubsection{O comportamento do espectro sob perturbação do operador}

Seja X um espaço normado complexo. Definiremos, agora, o espectro de um operador $A \in L(X)$. Recordemos que $L(X)$ é o espaço de todos os operadores lineares limitados $A$ de $X$ em $X$, munido da norma de operador. A topologia gerada por essa norma é chamada de topologia uniforme sobre $L(X)$, e qualquer noção de convergência e proximidade a seguir será com respeito à essa topologia.

Seja $A \in L(X)$. O conjunto resolvente $\rho(A)$ de $A$ consiste de todos números complexos $\lambda$ tais que (i) $(\lambda I-A)$ é um automorfismo do espaço $X$ e (ii) seu inverso $(\lambda I-A)^{-1}$ é limitado. Quando $X$ for um espaço de Banach, a condição (ii) será desnecessária, visto que, pelo Teorema da aplicação aberta, toda bijeção $T \in L(X)$ possui inversa limitada. Doravante, em vez de $(\lambda I-A)$, escreveremos somente $(\lambda-A)$. O inverso $(\lambda-A)^{-1}$ deste operador é chamado de resolvente e é comumente denotado por $R(\lambda, A)$.

O complementar do conjunto resolvente $\rho(A)$ é chamado de espectro de $A$; denotálo-emos por $\sigma(A)$.

De agora adiante, assumiremos que X é completo.

Proposição $3.7 O$ conjunto resolvente $\rho(A)$ é aberto e contém o conjunto $|\lambda|>\|A\|$. Portanto, $\sigma(A)$ é um subconjunto compacto do plano. Além disso, o espectro de um operador linear limitado é sempre não vazio.

DEMONSTRAÇÃo. Ver [41], págs. 190-192.

O espectro $\sigma(A)$ contém todos os autovalores de $A$, que são aqueles $\lambda \in \mathbf{C}$ tais que o núcleo $\operatorname{Ker}(\lambda-A)$ é não-trivial, i.é., contém pelo menos um vetor não-nulo. Todo elemento deste núcleo é, por definição, um autovetor de $A$ associado ao autovalor $\lambda$. Diremos que um autovalor $\lambda$ é simples se a dimensão de seu autoespaço generalizado

$$
G(\lambda)=\bigcup_{n=1}^{\infty} \operatorname{Ker}(\lambda-A)^{n}
$$

for igual a 1 . É fácil ver que $G(\lambda)$ é sempre um espaço vetorial - mesmo que $\lambda$ não seja simples -, pois a sequência de subespaços cuja reunião dá $G(\lambda)$ é crescente e encaixada. Além disso, $G(\lambda)$ possui dimensão finita precisamente quando existe um $n_{0}$ tal que

$$
\operatorname{Ker}(\lambda-A)^{n}=\operatorname{Ker}(\lambda-A)^{n_{0}},
$$


para todo $n \geq n_{0}$. Deste modo, o autovalor $\lambda$ será simples se, e somente se, (i) o núcleo $K_{\lambda}=\operatorname{Ker}(\lambda-A)$ possuir dimensão finita igual a 1 e (ii) sempre que $(\lambda-A) w$ pertencer à $K_{\lambda}$, tivermos $w \in K_{\lambda}$.

Se o que pretendemos é determinar o menor disco fechado que contém $\sigma(A)$, então podemos usar o raio espectral $r(A)$ de $A$, que, por definição, é o supremo dos $|\lambda|$, com $\lambda$ variando em $\sigma(A)$.

Proposição $3.8 r(A)=\lim _{n \rightarrow \infty}\left\|A^{n}\right\|^{\frac{1}{n}}$.

DEMONSTRAÇÃO. Ver [41], pág. 192.

\subsubsection{Analiticidade de auto-valores simples}

Agora estudaremos a variação do espectro mediante perturbações do operador original. Seja $A_{0} \in L(X)$ e suponhamos que $A_{0}$ tenha um autovalor simples e isolado $\lambda_{0}$, com autovetor associado $w_{0} \neq 0$. Podemos decompor o espectro de $A_{0}$ como uma reunião disjunta

$$
\sigma\left(A_{0}\right)=K_{0} \cup\left\{\lambda_{0}\right\}
$$

onde $K_{0}$ é um subconjunto compacto de $\mathbf{C}$. Agora consideremos dois abertos disjuntos $V$ e $W$ do plano, contendo $K_{0}$ e $\lambda$, respectivamente. Então

Proposição 3.9 Para todo operador linear limitado A suficientemente próximo de $A_{0}$, o espectro de A se decompõe como uma reunião disjunta

$$
\sigma(A)=K \cup\{\lambda\}
$$

onde $K$ é um compacto contido em $V$ e $\lambda \in W$ é um autovalor simples de A.

Deste modo, cada $A$ numa vizinhança $\mathscr{N}_{0}$ de $A_{0}$ possui um único autovalor simples $\lambda(A)$ em $W$. Sobre a regularidade da função $A \mapsto \lambda(A)$ afirmamos que

Teorema 3.3 Podemos tomar $\mathscr{N}_{0}$ de modo que cada $A$ em $\mathscr{N}_{0}$ possua um autovetor $w=w(A) \neq 0$ associado à $\lambda(A)$, com $w\left(A_{0}\right)=w_{0}$, sendo ambas $\lambda(A)$ e $w(A)$ analíticas como função de $A \in \mathscr{N}_{0}$.

Referências: O material necessário para se demonstrar estes dois resultados pode ser encontrado em [27]. Uma referência alternativa é [4]. O caso em que $X$ possui dimensão finita pode ser resolvido com o Teorema da função implícita. Já a abordagem para o caso de dimensão infinita é substancialmente diferente; a seguir, apresentaremos uma breve discussão com o intuito de oferecer ao leitor uma pequena amostra destas técnicas. Não se trata de uma demonstração do Teorema 3.3.

Escolhemos uma curva de Jordan $\gamma$ contida no conjunto resolvente $\rho\left(A_{0}\right)$ com a propriedade de que $\lambda_{0}$ está no seu interior $I(\gamma)$ e que o restante do espectro $K_{0}$ está em seu exterior - que é a componente ilimitada do complemento $\mathbf{C} \backslash \gamma$; ver Teorema 
da curva de Jordan. A orientação de $\gamma$ deve ser positiva. Usando as idéias do Cálculo Funcional, consideramos as integrais do resolvente

$$
\frac{1}{2 \pi i} \int_{\gamma}(\lambda-A)^{-1} d \lambda
$$

que estão bem definidas para todo $A$ suficientemente próximo de $A_{0}$ (ver Prop. anterior). A expressão acima define um operador linear limitado $P=P\left(\lambda_{0}, A\right)$ de $L(X)$ que satisfaz às seguintes propriedades: (i) $P$ é uma projeção, ou seja, $P^{2}=P$ e $\|P\|=1$; (ii) ambos os subespaços $P X$ e $(1-P) X$ são fechados e invariantes ${ }^{8}$ por $A$; e (iii) ao considerarmos separadamente as restrições de $A$ a estes dois subespaços invariantes (na ordem que os escrevemos), obtemos dois operadores lineares limitados cujos espectros são $\{\lambda\}$ e $K$, respectivamente (onde $\sigma(A)=K \cup\{\lambda\}$ ).

Outro resultado relacionado às projeções $P\left(\lambda_{0}, A\right)$ é que $P\left(\lambda_{0}, \cdot\right)$ é holomorfa numa vizinhança do operador $A_{0}$, e que o espectro $\{\lambda\}$ da restrição $\left.A\right|_{P\left(\lambda_{0}, A\right) X}$ varia holomorfamente com respeito à $A$. Maiores detalhes e resultados subsequêntes podem ser encontrados na literatura que indicamos acima.

\subsubsection{Operador de Transferência}

Sejam X um espaço métrico compacto, e $T$ uma transformação expansora deste espaço. Diremos que $T$ é topologicamente mixing quando, para todo aberto nãovazio $V$ de $X$, existir um $n \geq 1$ tal que $T^{n} V=X$. Uma medida de probabilidade Boreliana $\mu$ de $X$ será chamada de exata com respeito ao sistema $T$ quando os únicos Borelianos $A \subset X$ para os quais existem $A_{n} \in \mathscr{B}(X) \operatorname{com} A=T^{-n} A_{n}, n \geq 0$, forem aqueles de medida nula ou total $(\mu A=0$ ou $\mu A=1)$. Toda medida exata é ergódica com respeito à $T$. Se $\mu$ for uma medida Boreliana positiva sobre $X$, definiremos seu suporte supp $(\mu)$ como sendo o conjunto de todos os $x \in X$ tai que $\mu V>0$, para toda vizinhança de $V(x)$. Agora consideremos uma função complexa $\phi \in C^{\gamma}(X)$. Definimos

$$
L_{\phi}: C(X) \rightarrow C(X)
$$

por

$$
L_{\phi} g(x)=\sum_{y \in T^{-1}(x)}(\exp \phi(y)) g(y)
$$

Pelo modo como definimos transformações expansoras, vemos que $L_{\phi}$ leva função contínua em função contínua, ou seja, $L_{\phi}$ está bem definido. É fácil ver que $L_{\phi}$ é um operador linear limitado, o qual chamaremos de operador de transferência de Ruelle-Perron-Frobenius. Em manipulações, podemos evitar a repetição do somatório acima observando que

$$
L_{\phi} g=L_{0}(g \exp \phi)
$$

${ }^{8}$ Dizemos que um conjunto $B$ é invariante por uma função $f$ quando $f B \subset B$. 
onde 0 é o potencial nulo. Segue da definição de transformação expansora que, para todo $x_{0} \in X$, existe uma vizinhança $V\left(x_{0}\right)$ e funções contínuas $p_{1}(x), \ldots, p_{n}(x), n=$ $n\left(x_{0}\right)$, definidas em $V$ tais que

$$
T^{-1}(x)=\left\{p_{1}(x), \ldots, p_{n}(x)\right\},
$$

sempre que $x \in V$. Isso nos permite escrever $L_{\phi} g$ localmente na forma

$$
L_{\phi} g(x)=\left(\sum_{i=1}^{n}(g \exp \phi) \circ p_{i}\right)(x),
$$

para todo $x \in V$. O Teorema da Representação de Riesz 1.8 nos permite definir o operador dual

$$
L_{\phi}^{*}: M(X) \rightarrow M(X)
$$

de $L_{\phi}$, que, naturalmente, deve ${ }^{9}$ satisfazer $\left\langle\mu, L_{\phi} f\right\rangle=\left\langle L_{\phi}^{*}(\mu), f\right\rangle$, ou seja

$$
\int L_{\phi} f d \mu=\int f d\left(L_{\phi}^{*} \mu\right)
$$

sempre que $f \in C(X)$ e $\mu \in M(X)$.

Proposição 3.10 Sejam $C^{\gamma}(X ; \mathbf{C})=C^{\gamma}(X)$ e $C^{\gamma}(X ; \mathbf{R})$ o espaço das $\phi \in C^{\gamma}(X)$ cujos valores são reais. Então

$$
L_{\phi} C^{\gamma}(X ; \mathbf{C}) \subset C^{\gamma}(X ; \mathbf{C}) \text { e } L_{\psi} C^{\gamma}(X ; \mathbf{R}) \subset C^{\gamma}(X ; \mathbf{R}),
$$

se $\phi \in C^{\gamma}(X ; \mathbf{C})$ e $\psi \in C^{\gamma}(X ; \mathbf{R})$.

Referências: [13] e [38].

O próximo resultado é de fundamental importância em Formalismo Termodinâmico; ele relaciona os conceitos de pressão e entropia com propriedades espectrais do operador de transferência.

Teorema 3.4 (Ruelle-Perron-Frobenius) Seja $X$ um espaço métrico compacto e $T$ uma transformação expansora e topologicamente mixing. Seja $\phi \in C^{\gamma}(X)$ um potencial real para o sistema $(T, X)$, e coloquemos $\lambda=\exp P(\phi, T)$. Então existem uma função real $h>0$ em $C^{\gamma}(X)$ tal que $L_{\phi} h=\lambda h$, e uma única auto-medida de probabilidade positiva $v$ (i.é., $L_{\phi}^{*} v=\lambda v$ ). Se $g \geq 0, g \neq 0$ e $g \in C(X)$ satisfizer $L_{\phi} g=\lambda g$, então $\lambda=\tilde{\lambda}$ e g deverá ser um múltiplo constante $g=$ ch de $h$, para alguma $c>0$. Além disso, $d \mu=h d v$ é uma probabilidade exata para o sistema $T$, sendo também o único estado de equilíbrio. $O$ suporte de $\mu$ é todo $X$, e para toda $f \in C(X)$, vale a convergência

$$
\left\|\lambda^{-n} L_{\phi}^{n} f-h \int f d v\right\|_{\infty} \longrightarrow 0 .
$$

\footnotetext{
${ }^{9}\langle\mu, f\rangle=\int f d \mu$.
} 
Referências: Ver [13]. O enunciado presente nesta referência é aparentemente diferente do que damos acima, principalmente do ponto de vista de unicidade. De fato, o item (7) lá enunciado nada mais é que o princípio variacional, afirmando que o $\lambda$ deve ser igual a $\exp P(\phi)$, e que $\mu$ é o único estado de equilíbrio. A prova também mostra que qualquer auto-medida $v$ deve produzir este estado de equilíbrio através de $d \mu=h d v$. Mas como $h$ é estritamente positiva, $v$ deve ser única (mostre que $h d v_{1}=h d v_{2} \Rightarrow v_{1}=$ $v_{2}$ diferenciando a igualdade com relação à $m=v_{1}+v_{2}$ ). Também, não existe qualquer afirmação sobre o suporte da medida, mas o fato é que a técnica empregada durante todo o trabalho envolve a existência do Jacobiano, o que, para sistemas expansores e topologicamente mixing, faz com que supp $(\mu)=X$. Isso também é provado logo no início do capítulo III dessa mesma referência.

A referência [39] é bem mais completa que [13], mas o estilo desta última está mais próximo de nossa exposição.

As propriedades espectrais do operador de transferência enunciadas no Teorema acima nos permitem definir pressão para potenciais complexos e Hölder contínuos.

Observação. Os fatos que apresentaremos logo mais estão provados em [38], Cap. 4. O tipo de transformação $T$ estudada nessa referência é um caso específico definido num espaço de sequências. Afirmamos, porém, que os argumentos lá utilizados se estendem de modo fácil para o contexto abstrato que estamos estudando. Naturalmente, ao ler essa referência, o leitor necessita já ter um conhecimento prévio das técnicas envolvidas na demonstração do Teorema de Ruelle, o que pode ser feito com a leitura de [13]. Isso porque será necessário adaptar muitas desigualdades que lá aparecem. (O conjunto $F_{\theta}\left(X^{+}\right)$, por exemplo, deve ser trocado por $C^{\gamma}(X)$, e assim por diante).

Ainda supondo que $(T, X)$ é expansor e topologicamente mixing, consideramos um potencial complexo $\phi=u+i v$ em $C^{\gamma}(X)$. Então

Teorema 3.5 Ambas as partes real e imaginária de $\phi$ ainda estão em $C^{\gamma}(X)$, sendo (1) $O$ raio espectral $r\left(L_{\phi}\right)$ é menor ou igual a $\exp P(u, T)$. (Logo, o espectro $\sigma\left(L_{\phi}\right)$ é um subconjunto compacto do plano contido na bola fechada de raio $\exp P(u)$ ). Além disso,

(2) Se raio espectral for igual a $\exp P(u)$, então existirá um único ponto do espectro sobre o círculo $|z|=\exp P(u)$, o qual será necessariamente um auto-valor simples $\alpha$ de $L_{\phi} . O$ restante do espectro, i.é., $\sigma\left(L_{\phi}\right) \backslash \alpha$, está contido num disco fechado $|z| \leq s$ de raio s estritamente menor que $\exp P(u)$.

Das propriedades acima segue, em particular, que se $L_{\phi}$ não tiver auto-valores de módulo $\exp P(u)$, então o raio espectral do operador de transferência deverá ser estritamente menor que $\exp P(u)$.

\subsubsection{Definindo pressão para potenciais complexos}

Os resultados de perturbação que oferecemos anteriormente, juntamente com as propriedades acima, entrarão fortemente em nossa análise. Queremos definir $P(\phi, T)$ para 
potenciais complexos $\phi \in C^{\gamma}$. Enfatizamos, porém, que para podermos fazer isso, será necessário supor que $(T, X)$ seja expansor e topologicamente mixing. É importante que o leitor não se esqueça deste fato. No caso de conjuntos de Julia, por exemplo, poderemos aplicar as técnicas do Formalismo Termodinâmico somente quando a função racional for hiperbólica. (O tipo de métrica sobre o conjunto de Julia é irrelevante; lembremos que a pressão depende somente da topologia, e não da métrica).

Primeiramente, mostraremos que

I. A função $\phi \mapsto L_{\phi}$, definida em $C^{\gamma}(X)$ e tomando valores no espaço de Banach dos operadores lineares limitados de $C^{\gamma}$ nele mesmo, é holomorfa.

Provemos (I). Se $M: C^{\gamma} \hookleftarrow$ for linear e limitada, então definimos $L(M): C^{\gamma} \hookleftarrow$, também linear e limitada, por $L(M) f=L_{0}(M f)$. Observamos que $L(M)$ é uma função linear e contínua na variável $M$.

Dada $g \in C^{\gamma}(X)$, seja $M_{g}: C^{\gamma} \hookleftarrow$ o operador linear limitado de multiplicação por $g$, i.é., $M_{g} f=f g$. É fácil ver que $M_{g}$ é uma função linear e contínua de $g$.

Agora aplicamos a Proposição 1.18 e concluímos que $\phi \mapsto \exp \phi$ é holomorfa. Como toda função linear contínua é holomorfa, a composição

$$
L_{\phi}=L\left(M_{\exp \phi}\right)
$$

é uma função holomorfa na variável $\phi$, ou seja, acabamos de provar (I).

Segue da regra da cadeia que

$$
\frac{d L_{\phi}}{d \phi} g=L\left(M_{g \exp \phi}\right)
$$

de onde vem

$$
\frac{d L_{\phi+z \psi}}{d z}=L_{\phi+z \psi} \circ M_{\psi}
$$

Seja $\mathscr{S}$ o conjunto de todos os potenciais complexos $\phi \in C^{\gamma}(X)$ que verificam a seguinte propriedade: $\sigma\left(L_{\phi}\right)$ se decompõe como reunião disjunta de um compacto $K$ e um auto-valor simples $\lambda$, com $|\lambda|=r\left(L_{\phi}\right)$. Dos Teoremas 3.5 e 3.4 segue que toda $\phi \in C^{\gamma}(X ; \mathbf{R})$ está nessas condições. Mais ainda: se $\phi_{0} \in C^{\gamma}(X ; \mathbf{R})$, então toda $\phi \in C^{\gamma}(X ; \mathbf{C})$ suficientemente próxima de $\phi_{0}$ estará em $\mathscr{S}$, com o auto-valor simples $\lambda=\lambda(\phi)$ variando holomorfamente com $\phi$. Isso decorre dos resultados de perturbação que enunciamos e de (I). Para $\phi$ real, sabemos que $\lambda(\phi)>0$. Logo, se $\phi \in C^{\gamma}(X ; \mathbf{C})$ for suficiente próxima de $\phi_{0}$, teremos o ramo trivial de $\log \lambda(\phi)$ bem definido. Assim, existe um aberto $\mathscr{N}_{T} \supset C^{\gamma}(X ; \mathbf{R})$ - contido em $C^{\gamma}(X)$, obviamente - tal que $\log \lambda(\phi)$ está bem definida. Este valor é definido ${ }^{10}$ como sendo a pressão de $\phi$. Portanto,

Teorema 3.6 Seja $X$ um espaço métrico compacto e $T: X \hookleftarrow$ uma transformação expansora e topologicamente mixing. Então $P(\phi)$, como função de $\phi$, é holomorfa sobre $\mathscr{N}_{T}$.

\footnotetext{
${ }^{10}$ Definição que coincide com a anterior quando $\phi$ for real.
} 


\subsubsection{Cálculo do diferencial da pressão}

Acabamos de mostrar que $P(\phi)$ é analítica num subconjunto aberto $\mathscr{N}_{T}$ de $C^{\gamma}(X)$ contendo todos os potenciais reais $\phi \in C^{\gamma}(X)$. Agora vamos calcular explicitamente $D P(\phi)$, (ver definição de diferencial), com a hipótese de que o potencial $\phi \in C^{\gamma}(X) e ́$ real e $P(\phi)=0$. Em particular, o Teorema de Ruelle-Perron-Frobenius nos garante que o operador dual $L_{\phi}^{*}$ possui um ponto fixo $m$ (o auto-valor, neste caso, é $\lambda=\exp P(\phi)=$ 1) e o único estado de equilíbrio $\mu$ do sistema é dado por $d \mu=h d m$, onde $h$ é a autofunção de $L_{\phi}$ nas condições deste mesmo Teorema.

Considerando-se o estado de equilíbrio como funcional linear, mostraremos que $D P(\phi)=\mu$, ou seja

Proposição 3.11 Seja $\phi \in C^{\gamma}(X ; \mathbf{R})$ e T uma transformação expansora de topologicamente mixing de um espaço métrico compacto $X$. Para todo $g \in C^{\gamma}(X ; \mathbf{C})$, temos $D P(\phi) g=\int g d \mu$.

DemonstraÇÃo. Colocamos $p(z)=P(\phi+z g)$ e, para $\phi$ e $g$ fixadas nas condições acima, sabemos que $p(z)$ é analítica numa vizinhança da origem de $\mathbf{C}$. Vamos calcular $p^{\prime}(0)$. Pelo modo como definimos a pressão para potenciais complexos (que remete aos resultados de perturbação para o operador de transferência), encontramos uma função analítica $w(z)$, definida numa vizinhança do zero e tomando valores em $C^{\gamma}(X ; \mathbf{C})$, tal que

$$
L_{\phi+z g} w(z)=w(z) \exp p(z), w(0)=h,
$$

onde $h>0$ é o ponto fixo de $L_{\phi}$ nas condições do Teorema de Ruelle-Perron-Frobenius. Para calcular $p^{\prime}(0)$ diferenciamos (3.17) com respeito a $z$, e usando (3.16) concluímos que

$$
L_{\phi+z g}(g w(z))+L_{\phi+z g} w^{\prime}(z)=p^{\prime}(z) w(z) \exp p(z)+w^{\prime}(z) \exp p(z) .
$$

Em seguida fazemos $z=0$, obtendo

$$
L_{\phi}\left(g h+w^{\prime}(0)\right)=p^{\prime}(0) h+w^{\prime}(0)
$$

de onde

$$
\begin{aligned}
p^{\prime}(0) & =\int p^{\prime}(0) d \mu=\int p^{\prime}(0) h d m \\
& =\int L_{\phi}\left(g h+w^{\prime}(0)\right) d m-\int w^{\prime}(0) d m \\
& =\int g h d m+\int w^{\prime}(0) d m-\int w^{\prime}(0) d m \\
& =\int g d \mu
\end{aligned}
$$

Isso conclui a demonstração. 


\subsubsection{Fórmula de Bowen}

Apresentaremos uma importante relação entre pressão e dimensão de Hausdorff. Consideremos o caso de uma função racional hiperbólica $f$ da esfera de Riemann, de grau maior ou igual a dois, cujo conjunto de Julia $J(f)$ não contém $\infty$. Definimos

$$
\phi(z)=-\log \left|f^{\prime}(z)\right|,
$$

para $z \in J(f)$. Como $f$ não possui pontos críticos sobre seu conjunto de Julia, é fácil ver que $\phi$ é uma função contínua a valores reais sobre o espaço métrico compacto $J$. Logo, $t \phi$ é um potencial para o sistema $(f, J)$, para todo $t \geq 0$.

Teorema 3.7 (Fórmula de Bowen) Existe um único $t \geq 0$ satisfazendo $P(t \phi, f)=0$. Este número real é $>0$ e coincide com a dimensão de Hausdorff de J.

Esta é uma das mais surpreendentes aplicações do Formalismo Termodinâmico, onde um modelo físico é usado para solucionar um problema matemático. Ela foi desenvolvida por Rufus Bowen em [7].

Observação. Pode-se motrar que a dimensão $\delta$ do conjunto $J$ acima está no intervalo $(0,2)$, sendo sua medida de Hausdorff $h_{\delta}(J)$ estritamente positiva (ver [46]). Em particular, o conjunto de Julia de uma função racional hiperbólica sempre tem medida (Lebesgue de $\mathbf{C}$ ) nula.

Referências: A fórmula oferecida no Teorema 3.7 vale para uma classe mais geral de sistemas expansores, conhecida como repulsores conformes. Sua demonstração usa partições de Markov para semiconjugar a dinâmica da transformação à um subshift de um espaço de sequências (ver [40], pág. 44; bem como [50], pág. 53). Infelizmente, são ainda raras as boas exposições sobre o tema. Uma das exceções é [16], mas o caso específico de repulsor conforme considerado nessa referência não se aplica aos conjuntos de Julia. Um tratamento mais recente do assunto, englobando resultados de maior generalidade, é dado em [39], pág. 251. 


\section{Analiticidade real da dimensão de Hausdorff}

\subsection{Movimentos holomorfos parametrizados no disco} "We believe that holomorphic motions will provide simplified proofs of many fundamental results in com-
plex dynamics."

(F. GARDINER, Y. JIANG, AND Z. WANG - Holomorphic Motions and Related Topics)

\subsubsection{Extensão de Movimentos holomorfos}

Seja $\Lambda$ uma variedade complexa ${ }^{1}$ e conexa de dimensão $N$, e consideremos um subconjunto não-vazio qualquer $E$ da esfera de Riemann. Uma função

$$
h: \Lambda \times E \rightarrow \widehat{\mathbf{C}}
$$

será um movimento holomorfo de $E$, com ponto base $\lambda_{0} \in \Lambda$, quando (i) $h\left(\lambda_{0}, \cdot\right)$ for a identidade de $E$; (ii) $h(\lambda, \cdot)$ for injetiva para todo $\lambda$ em $\Lambda$; e (iii) cada $h(\cdot, z)$ for holomorfa. Diremos, então, que o movimento holomorfo $h(\lambda, z)$ está parametrizado em $\Lambda$. De modo equivalente, poderíamos ter definido um movimento holomorfo como sendo uma família de injeções $h_{\lambda}$ do conjunto $E$, como as mesmas propriedades de $h(\lambda, \cdot)$.

$\lambda$-Lema Seja $h(\lambda, z)$ um movimento holomorfo de um subconjunto $E \subset \widehat{\mathbf{C}}$, parametrizado sobre $\Lambda$. Então existe um único movimento holomorfo $H(\lambda, z)$ do fecho $\bar{E}$, parametrizado sobre $\Lambda$ e tal que $H=h$ sobre $\Lambda \times E$. Além disso, $H$ é uma função contínua de $\Lambda \times \bar{E}$, e cada $H_{\lambda}=H(\lambda, \cdot)$ é um homeomorfismo de $\bar{E}$ sobre sua imagem $H_{\lambda} \bar{E}$.

\footnotetext{
${ }^{1}$ Um espaço topológico Hausdorff e segundo enumerável, que localmente pode ser identificado com regiões de $\mathbf{C}^{N}$, com um altas cujas mudanças de coordenadas são equivalências conformes entre regiões de $\mathbf{C}^{N}$. Para detalhes, ver [36].
} 
Referências: $O$ resultado que enunciamos é um variante do $\lambda$-Lemma original, presente em [31]. Uma demonstração pode ser encontrada em [16], pág. 129. Para mais propriedades, ver [3] e [47]; ou mesmo [11] e [12], que oferecem demonstrações recentes do Teorema de Slodkowski [45] sobre extensão de movimentos holomorfos. Em [20] o leitor vai encontrar uma boa exposição sobre a evolução do assunto, que tiveram início em [31].

\subsubsection{Equicontinuidade de movimentos holomorfos}

Agora nos restringiremos ao caso em que $\Lambda$ é o polidisco disco unitário $\Delta^{N}(1)$ (ver índice de notação). O ponto base de $h(\lambda, z)$ será a origem de $\mathbf{C}^{N}$ e a norma que vamos considerar neste espaço é a do máximo: $|\lambda|_{0}=\max _{i}\left|\lambda_{i}\right|$. Diremos que o movimento holomorfo fixa um ponto $z$ se $h(\lambda, z)=z$, para todo $\lambda$. Quando os pontos $0,1, \infty$ estiverem em $E$ e forem fixados por $h$, diremos que $h$ é normalizado. Todo movimento holomorfo de um conjunto $E$ que contém pelo menos três pontos pode ser "normalizado"; isso é feito da seguinte forma. Sejam $z_{1}, z_{2}$ e $z_{3}$ pontos distintos de $E$. Para cada $\lambda$, existe uma transformação de Möbius ${ }^{2} B_{\lambda}$ que leva os três pontos distintos $h\left(\lambda, z_{1}\right)$, $h\left(\lambda, z_{2}\right)$ e $h\left(\lambda, z_{3}\right)$ em 0,1 e $\infty$, respectivamente. Definimos, então,

$$
\widetilde{h}(\lambda, z)=B_{\lambda} h\left(\lambda, B_{0}^{-1} z\right),
$$

que é um movimento holomorfo normalizado do conjunto $B_{0} E$. (As propriedades (i) e (ii) são trivialmente satisfeitas, enquanto que (iii) pode ser verificada usando a fórmula explícita de $B_{\lambda}(z)$, um quociente como em (4.2)).

Teorema 4.1 (Continuidade de Hölder) Seja $h(\lambda, z)$ um movimento holomorfo normalizado de um subconjunto fechado $E \subset \mathbf{C}$, parametrizado no polidisco unitário $\Delta^{N}(1)$, e com ponto base $\lambda=0$. Então, dado $R>0$, existe $C>0$ tal que para $|z|<R$ $e|w|<R$ temos

$$
\left|h_{\lambda}(z)-h_{\lambda}(w)\right| \leq C|z-w|^{\frac{1-|\lambda|_{0}}{1+\left.\lambda\right|_{0}}}
$$

para qualquer parâmetro $\lambda \in \Delta^{N}(1)$, onde $h_{\lambda}=h(\lambda, \cdot)$.

Referências: Este resultado é um Corolário da demonstração do $\lambda$-Lema (ver [16], págs. 130 e 131). Outras referências: [20], pág. 20; [19], pág. 13.

O resultado acima nos diz que, sobre conjuntos limitados, as injeções $h_{\lambda}$ do movimento holomorfo são aplicações Hölder contínuas com mesma constante de Hölder $C$.

Gostaríamos de estender este resultado para movimentos holomorfos quaisquer. Para o nosso estudo, será suficiente considerar o caso de um movimento holomorfo $h(\lambda, z)$ de um subconjunto compacto $E \subset \mathbf{C}$, tomando valores em $\widehat{\mathbf{C}}$, parametrizado no polidisco unitário $\Delta^{N}(1)$, e com ponto base $\lambda=0$. Em linhas gerais, o que faremos

\footnotetext{
${ }^{2}$ Para uma exposição destas transformações, consultar a seção sobre funções racionais.
} 
é utilizar o procedimento padrão para normalizar este movimento holomorfo e aplicar (4.1). Depois disso, todo nosso esforço será para trasportar a desigualdade obtida para o movimento holomorfo original, obtendo como resultado a Proposição 4.1.

Vamos assumir, como hipótese adicional, que E possui pelo menos três pontos. ${ }^{3}$

Dado $z_{1}$ em $E$, escolhemos um compacto $K$ propriamente contido em $E$, cujo interior em $E$ é não-vazio e contém $z_{1}$. Em seguida, tomamos outros dois pontos $z_{2}, z_{3}$ de $E$, com $z_{3} \notin K$. Para cada $\lambda$ no polidisco unitário, seja $B_{\lambda}$ única transformação de Möbius levando a tripla $h_{\lambda}\left(z_{1}\right), h_{\lambda}\left(z_{2}\right), h_{\lambda}\left(z_{3}\right)$ em $1,0, \infty$, respectivamente. Usando a compacidade de $K$ e propriedades específicas do movimento holomorfo, escolhemos um aberto $V$ de $\mathbf{C}$ contendo $K$ e um $\delta>0$ tal que para $|\lambda|_{0} \leq \delta$, tenhamos $h_{\lambda} K \subset V$ e $h_{\lambda}\left(z_{3}\right) \notin V$. Logo, quando $|\lambda|_{0} \leq \delta$, nenhum dos pontos $h_{\lambda}\left(z_{1}\right), h_{\lambda}\left(z_{2}\right), h_{\lambda}\left(z_{3}\right)$ será infinito, valendo

$$
B_{\lambda}(z)=\frac{\left(z-h_{\lambda} z_{2}\right)}{\left(z-h_{\lambda} z_{3}\right)} \frac{\left(h_{\lambda} z_{1}-h_{\lambda} z_{3}\right)}{\left(h_{\lambda} z_{1}-h_{\lambda} z_{2}\right)} .
$$

Com essa forma explícita, vemos também que, para qualquer $\delta^{*}>0$ ligeiramente maior que $\delta$, a aplicação $(\lambda, z) \mapsto B_{\lambda} z$ é holomorfa sobre $\left\{|\lambda|_{0}<\delta^{*}\right\} \times V$. Notemos que, como $h(\lambda, z)$ é contínua na variável $(\lambda, z)$, ambos os conjuntos

$$
E_{\delta}=\bigcup_{|\lambda|_{0} \leq \delta} h_{\lambda}(K), \quad F_{\delta}=\bigcup_{|\lambda|_{0} \leq \delta} B_{\lambda} h_{\lambda}(K)
$$

são compactos, sendo $E_{\delta} \subset V$ e, consequentemente, $\infty \notin F_{\delta}$.

Afirmação. Podemos escolher $\delta>0$ suficientemente pequeno, de modo que exista um aberto $V_{\delta}$ de $\mathbf{C}$ contendo $F_{\delta}$, tal que $B_{\lambda}^{-1} V_{\delta} \subset V$, sempre que $|\lambda|_{0} \leq \delta$.

Com efeito, é fácil verificar a partir da expressão da inversa $B_{\lambda}^{-1}(z)$ - obtida diretamente de (4.2) - que se $B_{\lambda_{0}}^{-1}\left(z_{0}\right)$ for diferente de $\infty$ num ponto $\left(\lambda_{0}, z_{0}\right)$, então o mesmo será verdadeiro numa vizinhança de $\left(\lambda_{0}, z_{0}\right)$. Como consequência, $(\lambda, z) \mapsto B_{\lambda} z$, será contínua neste ponto.

Observemos que $B_{0} K$ é compacto e está contido em $F_{\delta}$. Naturalmente, $B_{0}^{-1}$ leva este conjunto sobre sobre $K$, que por sua vez está contido em $V$. Dado $z_{0} \in K$, existem $\eta_{z_{0}}$ e uma vizinhança $W\left(z_{0}\right)$ tais que $B_{\lambda}^{-1}(z) \in V$, para $|\lambda|_{0} \leq \eta$ e $z \in W$. Mas como $B_{0} K$ é compacto, podemos considerar apenas um número finito de vizinhanças $W\left(z_{0}\right)$-com um número finito de pontos $z_{0}-$ e tomar $\delta$ como sendo menor que todos os valores $\eta_{z_{0}}$ correspondentes. Concluímos daí que existe um aberto $W_{1}$ contendo $B_{0} K$, tal que $B_{\lambda}^{-1} W_{1} \subset V$, para $|\lambda|_{0} \leq \delta$. É claro, podemos escolher $\delta>0$ ainda menor, de modo que $F_{\delta}$ esteja contido em $W_{1}$. A afirmação segue $\operatorname{com} V_{\delta}=W_{1}$.

Recordemos que $\delta$ é o mesmo, desde o início da demonstração, tendo sido diminuído de modo a satisfazer às propriedades descritas até o momento. Com isso, se $\delta^{*}$ for

\footnotetext{
${ }^{3}$ Nas aplicações, $E$ será o conjunto de Julia de uma função racional hiperbólica, o qual contém infinitos pontos. Um dos modos de se verificar este fato - que pode ser deduzido com argumentos mais elementares - é lembrar que a dimensão de Hausdorff de tais conjuntos é sempre positiva (ver a observação que segue a Fórmula de Bowen).
} 
ligeiramente maior que $\delta$, também $(\lambda, z) \mapsto B_{\lambda}^{-1}(z)$ é holomorfa em $\left\{|\lambda|_{0}<\delta^{*}\right\} \times V_{\delta}$. A imagem dessa aplicação está contida em $V$.

O movimento holomorfo normalizado $B_{\lambda} h\left(\lambda, B_{0}^{-1} z\right)$ do conjunto limitado $B_{0} K$ satisfaz

$$
\left|B_{\lambda} h\left(\lambda, B_{0}^{-1} z\right)-B_{\lambda} h\left(\lambda, B_{0}^{-1} w\right)\right| \leq C \mid z-w^{\frac{1-|\lambda|_{0}}{1+|\lambda|_{0}}}
$$

sempre que $\lambda \in \Delta^{N}(1)$ e $z, w \in B_{0} K$. Para transportar essa desigualdade para o movimento holomorfo original, vamos mostrar que $B_{\lambda}^{-1}$ é Lipschitziana no compacto $F_{\delta}$, com constante de Lipschitz independente de $\lambda$. Para tanto, escolhemos um aberto intermediário $V_{2} \supset F_{\delta}$, cujo fecho $\bar{V}_{2}$ é compacto e contido em $V_{\delta}$. Seja $M$ o máximo de $\left|\left(B_{\lambda}^{-1}\right)^{\prime} \zeta\right|$ tomado sobre $\left\{|\lambda|_{0} \leq \delta\right\} \times \bar{V}_{2}$. (Do Teorema de Hartogs, $\left(B_{\lambda}^{-1}\right)^{\prime}(\zeta)$ é holomorfa em $(\lambda, \zeta)$; em particular, contínua). Escolhemos $\eta \in(0,1)$ de modo que, para quaisquer $z, w \in F_{\delta}$ com $|z-w|<\eta$, o segmento $[z, w]$ esteja contido em $\bar{V}_{2}$. (Ver Teorema do Valor Médio). Assim, sempre que $z, w \in F_{\delta} \mathrm{e}|z-w|<\eta$, teremos

$$
\left|B_{\lambda}^{-1}(z)-B_{\lambda}^{-1}(w)\right| \leq M|z-w| .
$$

Substituímos esta última desigualdade em (4.3), observando que ambos $B_{\lambda} h\left(\lambda, B_{0}^{-1} z\right)$ e $B_{\lambda} h\left(\lambda, B_{0}^{-1} w\right)$ estarão em $F_{\delta}$ e a uma distância menor que $\eta$ um do outro, sempre que $|\lambda|_{0} \leq \delta$, bastando que se tome $|z-w|<\kappa_{0}$, para alguma constante $\kappa_{0}>0$ suficientemente pequena e independente de $\lambda$ (para tanto, usar a continuidade uniforme do movimento holomorfo em subonjuntos compactos de seu domínio). Deste modo,

$$
\begin{aligned}
\left|h\left(\lambda, B_{0}^{-1} z\right)-h\left(\lambda, B_{0}^{-1} w\right)\right| & =\left|B_{\lambda}^{-1} B_{\lambda} h\left(\lambda, B_{0}^{-1} z\right)-B_{\lambda}^{-1} B_{\lambda} h\left(\lambda, B_{0}^{-1} w\right)\right| \\
& \leq M\left|B_{\lambda} h\left(\lambda, B_{0}^{-1} z\right)-B_{\lambda} h\left(\lambda, B_{0}^{-1} w\right)\right| \\
& \leq M C|z-w|^{\frac{1-|\lambda|_{0}}{1+|\lambda|_{0}}}
\end{aligned}
$$

sempre que $z, w \in B_{0} K, \operatorname{com}|z-w|<\kappa_{0}$ e $|\lambda|_{0} \leq \delta$.

Todo ponto de $K$ é da forma $B_{0}^{-1} z$, para algum $z \in B_{0} K$. Além disso, um argumento padrão nos informa que $B_{0}$ é uma aplicação bi-Lipschitz de $K$ sobre $B_{0} K$. Tendo em vista (4.5), afirmamos então que existem constantes $C_{0}$ e $\eta_{1}>0$ tais que

$$
|h(\lambda, z)-h(\lambda, w)| \leq C_{0}|z-w|^{\frac{1-|\lambda|_{0}}{1+|\lambda|_{0}}},
$$

sempre que $z, w \in K,|z-w|<\eta_{1}$ e $|\lambda|_{0} \leq \delta$.

Agora retornamos ao início de toda essa argumentação e recordamos que o interior de $K$ em $E$ contém o ponto $z_{1}$, que foi tomado arbitrariamente. Podemos, assim, formar uma cobertura aberta $\mathscr{C}$ de $E$ com os interiores destes compactos e extrair uma subcobertura finita. Cada elemento desta cobertura finita determina constantes $C_{0}, \eta_{1}, \delta$, como acima. Tomamos $\delta^{*}$ como sendo o mínimo de todos os $\delta$ 's; $\eta^{*}>0$ menor que o número de Lebesgue de $\mathscr{C}$ e menor que qualquer dos $\eta_{1}$ provenientes da cobertura finita. Em seguida, escolhemos $C_{0}^{*}$ como sendo o máximo dos $C_{0}$ 's. Concluímos que 


$$
|h(\lambda, z)-h(\lambda, w)| \leq C_{0}^{*}|z-w|^{\frac{1-|\lambda|_{0}}{1+|\lambda|_{0}}},
$$

para $|\lambda|_{0} \leq \delta^{*}, z, w \in E$ e $|z-w|<\eta^{*}$. É claro que, usando a continuidade do movimento holomorfo, podemos suprimir a constante $\eta^{*}$ acima, bastando que se tome $C_{0}^{*}$ um pouco maior. Resumimos o que acabamos de deduzir na seguinte

Proposição 4.1 Seja $h(\lambda, z)$ um movimento holomorfo de um compacto $E \subset \mathbf{C}$, parametrizado em $\Delta^{N}(1)$, e com ponto base 0 . Então existem $\delta>0$ e uma constante $C>0$ tais que $h_{\lambda}(E) \subset \mathbf{C} e$

$$
|h(\lambda, z)-h(\lambda, w)| \leq C|z-w|^{\frac{1-|\lambda|_{0}}{1+\left.\lambda\right|_{0}}}
$$

para quaisquer $z, w \in E$, desde que $|\lambda|_{0} \leq \delta$.

\subsection{J-Estabilidade de funções racionais hiperbólicas}

Seja $\Lambda$ uma variedade complexa e conexa de dimensão $N$. Uma família de endomorfismos $f_{\lambda}$ da esfera de Riemann será uma família holomorfa sempre que $f(\lambda, z)=f_{\lambda} z$ for uma função holomorfa da variedade produto $\Lambda \times \widehat{\mathbf{C}}$. Decorre da Proposição 1.21 que cada $f_{\lambda}$ é uma função racional. Vamos sempre supor que cada uma das funções racionais $f_{\lambda}$ possui grau maior ou igual a dois.

Cada função racional $f_{\lambda}$ da família holomorfa possui um conjunto de Julia $J_{\lambda}$, como definido na seção 2.2. Diremos que estes conjuntos $J_{\lambda}$ se movem holomorfamente num ponto $\lambda_{0}$ de $\Lambda$ se existir um movimento holomorfo $h_{\lambda}$ do conjunto $J_{\lambda_{0}}$, parametrizado nalguma vizinhança $N\left(\lambda_{0}\right) \subset \Lambda$, e com ponto base $\lambda_{0}$, tal que cada $h_{\lambda}$ leva $J_{\lambda_{0}}$ homeomorfamente sobre $J_{\lambda}$, com

$$
h_{\lambda} f_{\lambda_{0}}(z)=f_{\lambda} h_{\lambda}(z),
$$

ou seja, $h_{\lambda}$ é uma conjugação de $\left(f_{\lambda_{0}}, J_{\lambda_{0}}\right)$ para $\left(f_{\lambda}, J_{\lambda}\right)$.

Teorema 4.2 Os conjuntos de Julia $J_{\lambda}=J\left(f_{\lambda}\right)$ de uma família holomorfa de funções racionais hiperbólicas $f_{\lambda}, \lambda \in \Lambda$, se movem holomorfamente em todo ponto $\lambda_{0}$ de $\Lambda$. Além disso, se $\varphi$ for uma carta local definida numa vizinhança conexa $U$ do ponto $\lambda_{0}$, então poderemos tomar $N\left(\lambda_{0}\right)=U$ (ver definição acima).

DEMONSTRAÇÃO. Seja $\varphi$ um sistema de coordenadas locais definido numa vizinhança conexa do ponto $\lambda_{0}$ e coloquemos

$$
g(\mu, z)=f\left(\varphi^{-1}(\mu), z\right),
$$

que é uma família holomorfa de funções racionais hiperbólicas (de fato, apenas uma raparametrização de $f_{\lambda}$ na região $\varphi(U)$ contida em $\mathbf{C}^{N}$ ). Mostraremos que os conjuntos de Julia

\footnotetext{
${ }^{4}$ A palavra sobre quer dizer sobrejetividade.
} 


$$
J\left(g_{\mu}\right)=J\left(f_{\varphi^{-1}(\mu)}\right)
$$

se movem holomorfamente no ponto $\mu_{0}=\varphi\left(\lambda_{0}\right)$ através de um movimento holomorfo $h_{\mu}$, parametrizado na região $U^{\prime}=\varphi(U)$. À este movimento sobre $U^{\prime}$ corresponde um único movimento holomorfo $h_{\lambda}^{1}=h_{\varphi \lambda}$, parametrizado em todo o sistema de coordenadas $(U, \varphi)$, satisfazendo às condições do enunciado do teorema. Deste modo, não há perda de generalidade em assumirmos que a própria variedade $\Lambda$ seja um aberto e conexo de $\mathbf{C}^{N}$.

Fixemos um $\lambda_{0}$ em $\Lambda$. Primeiramente, construímos um movimento holomorfo do conjunto dos pontos periódicos repulsores de $f_{\lambda_{0}}$, já que eles são densos do conjunto de Julia. Seja $z_{0}$ um ponto periódico repulsor de $f_{\lambda_{0}}$, com período $p$. Para $\lambda$ suficientemente próximo de $\lambda_{0}$, existem pontos periódicos repulsores $z_{0}(\lambda)$ de $f_{\lambda}$, todos com o mesmo período $p$, de modo que $z_{0}(\lambda)$ seja um função holomorfa na variável $\lambda$, com $z_{0}\left(\lambda_{0}\right)=z_{0}$. Isso é feito a partir do Teorema da função implícita aplicado à

$$
F(\lambda, z)=f_{\lambda}^{p}(z)-z
$$

Seja $V$ o domínio da função $z_{0}(\lambda)$. Afirmamos que esta função possui uma extensão holomorfa à todo o $\Lambda$ - extensão esta que continuaremos denotando por $z_{0}(\lambda)-$ de modo que, assim como no caso em que $\lambda \in V, z_{0}(\lambda)$ continue sendo um ponto periódico repulsor de período $p$ de $f_{\lambda}$, para todo $\lambda \in \Lambda$. Observemos que se $\lambda \in V$, $\lambda \rightarrow \lambda_{*}$ e $z_{0}(\lambda) \rightarrow z_{0}\left(\lambda_{*}\right)$, então por continuidade concluímos que $z_{0}\left(\lambda_{*}\right)$ deve ser periódico de período $p$. Do mesmo modo,

$$
\left|\left(f_{\lambda_{*}}^{p}\right)^{\prime} z_{0}\left(\lambda_{*}\right)\right| \geq 1
$$

mas como $f_{\lambda_{*}}$ não possui ciclos indiferentes (Proposição 2.8), $z_{0}\left(\lambda_{*}\right)$ deve ser um ponto periódico repulsor; logo, pelo Teorema da Função Implícita, existe um prolongamento da solução $z_{0}(\lambda)$ numa vizinhança de $z_{0}\left(\lambda_{*}\right)$. Usando continuação analítica, concluímos que existe uma extensão definida em todo $\Lambda$.

O segundo passo para se construir o movimento holomorfo será provar a condição de injetividade; preliminarmente, mostraremos que se $z_{0}$ e $z_{1}$ forem pontos periódicos repulsores distintos de $f_{\lambda_{0}}$, então deveremos ter

$$
z_{0}(\lambda) \neq z_{1}(\lambda)
$$

por todo o conjunto $\Lambda$. De fato, suponhamos que $z_{0}\left(\lambda_{1}\right)=z_{1}\left(\lambda_{1}\right)$, para algum $\lambda_{1}$. Ambos $z_{0}(\lambda)$ e $z_{1}(\lambda)$ são pontos periódicos repulsores de $f_{\lambda}$, com períodos $p$ e $q$, respectivamente. Segue-se que $z_{0}(\lambda)$ e $z_{1}(\lambda)$ são local e implicitamente definidas através da igualdade

$$
F(\lambda, z)=f_{\lambda}^{p q}(z)-z=0,
$$

para $\lambda$ próximo de $\lambda_{1}$. Pela regra do produto $(2.1)$, o ponto $z_{0}\left(\lambda_{1}\right)=z_{1}\left(\lambda_{1}\right)$ é periódico e repulsor; logo, a função acima está nas condições do Teorema da função implícita, e concluímos daí que $z_{0}(\lambda)$ e $z_{1}(\lambda)$ devem coincidir localmente. $\mathrm{O}$ mesmo argumento, 
aliás, nos mostra que o conjunto de todos os $\lambda \in \Lambda$ nos quais $z_{0}(\lambda)=z_{1}(\lambda)$ é simultaneamente aberto e fechado e, portanto, igual à $\Lambda$. Em particular, fazendo $\lambda=\lambda_{1}$ encontramos $z_{0}=z_{1}$, o que equivale a dizer que $z_{0} \mapsto z_{0}(\lambda)$, para $\lambda$ fixo, é injetora.

Agora definimos explicitamente o movimento holomorfo

$$
\begin{gathered}
h(\lambda, z): \Lambda \times E_{\lambda_{0}} \rightarrow \widehat{\mathbf{C}}, \\
h(\lambda, z)=z(\lambda),
\end{gathered}
$$

do conjunto $E_{\lambda_{0}}$ dos pontos periódicos repulsores de $f_{\lambda_{0}}$. O ponto base deste movimento é $\lambda_{0}$. Usando o $\lambda$-Lema, encontramos um movimento holomorfo do conjunto de Julia $J_{\lambda_{0}}=\overline{E_{\lambda_{0}}}$, o qual continuaremos denotando por $h(\lambda, z)$. Pelo $\lambda$-Lema, as injeções $h_{\lambda}=h(\lambda, \cdot)$ são obviamente homeomorfismos sobre as imagens $h_{\lambda}\left(J_{\lambda_{0}}\right)$. Para completar a demonstração, falta apenas mostrar que $h_{\lambda} J_{\lambda_{0}}$ é exatamente $J_{\lambda}$, e que

$$
h_{\lambda} f_{\lambda_{0}}(z)=f_{\lambda} h_{\lambda}(z) \text {, }
$$

para todo $z$ em $J_{\lambda_{0}}$. Primeiramente, verificaremos a última igualdade, a qual segue de um argumento um pouco mais geral; de fato, se $z_{0}, z_{1}, \ldots, z_{p-1}$ for um ciclo repulsor, então $z_{0}(\lambda), \ldots, z_{p-1}(\lambda)$ também será um ciclo repulsor, com o mesmo período do ciclo anterior. ${ }^{5}$ Deste modo, para todo $z_{0} \in E_{\lambda_{0}}$,

$$
h_{\lambda} f_{\lambda_{0}}\left(z_{0}\right)=h_{\lambda} z_{1}=z_{1}(\lambda)=f_{\lambda_{0}} z_{0}(\lambda)=f_{\lambda_{0}} h_{\lambda}\left(z_{0}\right) \text {. }
$$

Para mostrar que $h_{\lambda} J_{\lambda_{0}}=J_{\lambda_{1}}$, para todo $\lambda_{1}$ em $\Lambda$, observamos que poderíamos ter começado a construção do movimento holomorfo a partir do ponto base $\lambda_{1} \mathrm{em}$ vez de $\lambda_{0}$, concluindo que, para todo ponto periódico repulsor $w_{1}$ em $E_{\lambda_{1}}$, existe uma função holomorfa $w_{1}(\lambda) \in E_{\lambda}$ na variável $\lambda$, definida em todo $\Lambda$. Seja $z_{0}=w_{1}\left(\lambda_{0}\right)$. Ambas $z_{0}(\lambda)$ e $z_{1}(\lambda)$ devem coincidir sobre a região $\Lambda$, o que pode ser verificado com um argumento que vimos usando repetidamente ao longo desta demonstração. Segue então que todo ponto $w_{1}$ de $E_{\lambda_{1}}$ é a imagem $h_{\lambda} z_{0}=z_{0}\left(\lambda_{1}\right)$ de algum ponto $z_{0}$ de $E_{\lambda_{0}}$. Podemos, finalmente, usar o fato de que ambos os conjuntos de pontos periódicos repulsores $E_{\lambda_{0}}$ e $E_{\lambda_{1}}$ são densos em seus respectivos conjuntos de Julia, e concluir que $h_{\lambda} J_{\lambda_{0}}=J_{\lambda_{1}}$. (Será preciso usar a compacidade de $J_{\lambda_{0}}$ para verificar esse fato).

\subsection{Analiticidade real da dimensão de Hausdorff}

Nesta seção, empregaremos as seguintes notações:

$$
\begin{aligned}
& \Delta_{R}^{*}=\{z \in \mathbf{C}: 0<|z|<R\}, \\
& \Delta^{d}(r)=\left\{z \in \mathbf{C}^{d}:\left|z_{j}\right|<r\right\}
\end{aligned}
$$

\footnotetext{
${ }^{5}$ Use o resultado de unicidade do Teorema da função implícita para as funções $z_{1}(\lambda)$ e $f z_{0}(\lambda)$, concluindo que elas são iguais, primeiro localmente, e depois por todo o conexo $\Lambda$.
} 
$\mathrm{e}$

$$
\overline{\Delta^{d}}(r)=\left\{z \in \mathbf{C}^{d}:\left|z_{j}\right| \leq r\right\} .
$$

Reservaremos as letras para $z$ e $w$ para indicar elementos gerais de $\mathbf{C}^{d}$, enquanto que $x$ e $y$ serão sempre vetores de $\mathbf{C}^{d} \simeq \mathbf{R}^{d}+i \mathbf{R}^{d}$ cuja parte imaginária é nula.

Objetivo. Considerando-se a métrica $\widehat{d}$ da esfera de Riemann, e o conjunto de medidas de Hausdorff $\delta$-dimensionais definidas a partir dela, mostraremos que a função

$$
d(\lambda)=\operatorname{dim}_{\mathrm{H}} J\left(f_{\lambda}\right)
$$

é analítica real, onde $f_{\lambda}$ é uma família holomorfa de funções racionais hiperbólicas de grau $\geq 2$, indexadas numa variedade analítica complexa $\Lambda$ de dimensão $N$.

Como pretendemos usar a fórmula de Bowen para resolver este problema, vamos nos restringir ao caso em que o conjunto de Julia está contido no plano, ou seja, exclui o ponto $\infty$. No que segue, procuraremos justificar este argumento, garantindo que não há perda de generalidade.

Fixemos $\lambda_{0}$ em $\Lambda$ e suponhamos que $\infty$ esteja em $J_{\lambda_{0}}=J\left(f_{\lambda_{0}}\right)$. Como $J_{\lambda_{0}}$ é diferente de $\widehat{\mathbf{C}}$ (ver observação que segue a fórmula de Bowen), existe um ponto $a \in \mathbf{C}$ que está fora deste conjunto. A transformação de Möbius

$$
R(z)=\frac{1}{z-a}
$$

leva $a \mathrm{em} \infty$, e $\infty$ na origem. Assim, $R J_{\lambda_{0}}$ é um suconjunto compacto de plano, omitindo, portanto, o ponto no infinito. Mostraremos que $R$ é bi-Lipschitz com respeito à $\widehat{d}$; isso nos vai garantir que

$$
\operatorname{dim}_{\mathrm{H}} R J_{\lambda}=\operatorname{dim}_{\mathrm{H}} J_{\lambda}
$$

para todo $\lambda$ suficientemente próximo de $\lambda_{0}$.

Escolhemos três vizinhanças encaixadas $U_{1} \supset U_{2} \supset U_{3}$ de $a$ (de tamanho convenientemente pequeno) e outras três vizinhanças encaixadas $V_{1} \supset V_{2} \supset V_{3}$ de $\infty$. Seja $K_{2}$ o complementar em $\widehat{\mathbf{C}}$ da reunião de $V_{2} \operatorname{com} U_{2}$; e seja $K_{3}$ o complementar em $\widehat{\mathbf{C}}$ da reunião de $V_{3}$ com $U_{3}$. Ambos são subconjuntos compactos de $\mathbf{C}$, com $K_{2}$ contido no interior de $K_{3}$. Como $R^{\prime}(z)$ não possui pólos sobre $K_{3},\left|R^{\prime}(z)\right|$ é limitada por uma constante $M$ sobre este conjunto. Podemos aplicar o Teorema do Valor Médio para pontos $z, w$ suficientemente próximos um do outro, concluindo que existe um $\delta>0$ tal que

$$
|R(z)-R(w)| \leq M|z-w|
$$

sempre que $z, w \in K_{2}$ estiverem à uma distância menor que $\delta$ um do outro. Naturalmente, isso já mostra que $R$ é Lipschitz sobre $K_{2}$, com respeito à norma de $\mathbf{C}$. Pela Proposição 1.20, $R$ também é Lipschitz com respeito à $\widehat{d}$ sobre este mesmo conjunto. Em particular, $R$ é Lipschitz sobre o aberto 


$$
O=\left(\overline{V_{2}} \cup \overline{U_{2}}\right)^{c}
$$

Agora mostraremos que $R$ é Lipschitz sobre $U_{1}$. De acordo com a notação da Proposição 1.20 , temos

$$
d_{\varphi_{\infty}}(z, w)=|(z-a)-(w-a)| \leq|z-w|,
$$

para $z, w \in U_{2}$. Novamente, aplicamos a Proposição 1.20 e concluímos que $R$ é Lipschitz sobre $U_{2}$.

No caso de $V_{1}$, observamos ${ }^{6}$ que

$$
R \varphi_{\infty}^{-1}(z)=\frac{z}{1-a z}
$$

é uma transformação de Möbius com nenhum pólo próximo à zero, e, como tal, é Lipschitziana numa vizinhança da origem. Portanto, existe uma constante $C>0$ tal que

$$
\left|R \varphi_{\infty}^{-1}(z)-R \varphi_{\infty}^{-1}(w)\right| \leq C|z-w|=d_{\varphi_{\infty}}\left(\varphi_{\infty}^{-1}(z), \varphi_{\infty}^{-1}(w)\right) .
$$

A Proposição 1.20 nos garante que podemos escolher $V_{1}$ de modo que $R$ seja Lipschitz nessa vizinhança.

Notemos que $V_{1}, O$ e $U_{1}$ constituem uma cobertura aberta da esfera de Riemann, a qual possui um número de Lebesgue $\delta^{*}$, de onde concluímos que $R$ é Lipschitz para pontos $|z-w|<\delta^{*}$. Mas como $\widehat{\mathbf{C}}$ é compacta, $R$ deve ser Lischitziana globalmente.

De modo similar, mostra-se a mesma propriedade para a inversa $R^{-1}$. Assim, $R$ é, de fato, uma aplicação bi-Lipschitz, e como tal, preserva dimensão de Hausdorff.

Consideremos a família de funções racionais $g_{\lambda}=R f_{\lambda} R^{-1}$. É fácil ver que $K_{\lambda}=$ $R J_{\lambda}$ é invariante por $g_{\lambda}$. Mostraremos que, de fato, este é o conjunto de Julia de $g_{\lambda}$, e que $g_{\lambda}$ é uma família holomorfa de funções racionais hiperbólicas para $\lambda$ suficientemente próximo à $\lambda_{0}$.

Um ponto $z_{0}$ pertence ao conjunto de Fatou de $f_{\lambda}$ precisamente quando existe uma vizinhança $U\left(z_{0}\right)$ na qual a família de iterados $f_{\lambda}^{n}$ é normal. Se $f_{\lambda}^{n_{k}}$ for uma subsequência convergindo localmente uniformemente sobre $U$ para uma função holomorfa $f: U \rightarrow \widehat{\mathbf{C}}$, então a sequência correspondente $g_{\lambda}^{n_{k}}=R f_{\lambda}^{n_{k}} R^{-1}$ também convergirá localmente uniformemente sobre $R(U)$ (com respeito à métrica $\widehat{d}$ ). Com isso, concluímos que $R\left(z_{0}\right)$ estará no conjunto de Fatou de $g_{\lambda}$ sempre que $z_{0}$ estiver em $F\left(f_{\lambda}\right)$. Mas o fato é que $f_{\lambda}$ também é obtida de $g_{\lambda}$ através de $R^{-1}$, de onde segue a igualdade entre os conjuntos de Fatou e, consequentemente, entre os respectivos conjuntos de Julia.

Agora mostraremos que $g_{\lambda}$ é hiperbólica. Seja $\langle\cdot, \cdot\rangle$ uma métrica conforme definida numa vizinhança de $J_{\lambda}$. Então

$$
(z, w)_{p}=\left\langle D R^{-1}(z), D R^{-1}(w)\right\rangle_{R^{-1}(p)}
$$

define uma métrica conforme numa vizinhança de $K_{\lambda}$. Denotaremos a norma do espaço tangente em $p$ com respeito à $(\cdot, \cdot)$ por $\|\cdot\|_{R, p}$, ou, simplesmente, $\|\cdot\|_{R}$. Com o

\footnotetext{
${ }^{6}$ Ver índice de notação
} 
mesmo símbolo também denotaremos a norma $\|A\|_{R}$ de uma transformação linear entre espaços tangentes. Com isso, fica fácil verificar que, para todo $p \in K_{\lambda}$,

$$
\left\|D g_{\lambda}(p)\right\|_{R}=\left\|D f_{\lambda}\left(R^{-1} p\right)\right\|
$$

de onde segue a hiperbolicidade da família $g_{\lambda}$.

Usando a compacidade de $K_{\lambda_{0}}$ e o Teorema 4.2, concluímos que existe uma vizinhança $V\left(\lambda_{0}\right) \subset \Lambda$ tal que $K_{\lambda} \subset \mathbf{C}$, sempre que $\lambda \in V$.

Registraremos este resultado preliminar no

Lema 4.1 Seja $f(\lambda, z)$ uma família holomorfa de funções racionais hiperbólicas de $\widehat{\mathbf{C}}$, parametrizadas em alguma variedade analítica complexa $\Lambda$ de dimensão $N$. Dado $\lambda_{0} \in \Lambda$, existem uma transformação de Möbius bi-Lipschitz $R_{\lambda_{0}}$ e uma vizinhança $V\left(\lambda_{0}\right)$ tais que:

(i) $K_{\lambda}=R_{\lambda_{0}} J\left(f_{\lambda}\right)$ está contido em $\mathbf{C}$, para todo $\lambda \in V$;

(ii) $K_{\lambda}=J\left(g_{\lambda}\right)$, onde $g_{\lambda}=R_{\lambda_{0}} f_{\lambda} R_{\lambda_{0}}^{-1}$, sempre que $\lambda \in V$;

(iii) $g_{\lambda}, \lambda \in V$,é uma família holomorfa de funções racionais hiperbólicas.

Como já observamos, a dimensão de Hausdorff de $K_{\lambda}$ é a mesma de $J_{\lambda}$. Queremos mostrar uma propriedade local, i.é., que a função $d(\lambda)=\operatorname{dim}_{\mathrm{H}} K_{\lambda}$ é analítica real em $V$, onde $V$ é a vizinhança dada pelo Lema acima. Podemos tomar $V$ de modo que exista uma equivalência conforme entre $V$ e $\Delta^{N}(1)$, e em seguida reparametrizar o movimento holomorfo $h_{\lambda}: K_{\lambda_{0}} \rightarrow K_{\lambda}$ obtido do Teorema 4.2 substituindo $V$ pelo polidisco unitário $\Delta^{N}(1) \subset \mathbf{C}^{N}$, e redefinindo o ponto base como sendo $\lambda_{0}=0$.

Considerando-se o potencial $\phi_{\lambda}=-\log \left|g_{\lambda}^{\prime}(z)\right|$ do sistema $\left(g_{\lambda, K_{\lambda}}\right)$, a fórmula de Bowen nos diz que $d(\lambda)$ é dada implicitamente por

$$
P\left(d(\lambda) \phi_{\lambda}, g_{\lambda}\right)=0 \text {. }
$$

Para podermos aplicar os resultados de diferenciabilidade de $P(\phi)$, devemos eliminar a dependência de $g_{\lambda}$ na fórmula acima. Isso pode ser feito com o auxílio da Proposição 3.3; como $h_{\lambda}$ é uma conjugação topológica entre os conjuntos de Julia, temos

$$
P\left(d(\lambda) \phi_{\lambda} \circ h_{\lambda}, g_{0}\right)=0 .
$$

Definimos

$$
D(z, \lambda)=g_{\lambda}^{\prime}\left(h_{\lambda} z\right), \quad \psi_{\lambda}(z)=-\log |D(z, \lambda)|,
$$

para $z \in K_{0}$ e $\lambda \in \Delta^{N}(1)$, e observamos que $\psi_{\lambda}=\phi_{\lambda} \circ h_{\lambda}$. Das propriedades descritas para movimentos holomorfos decorrem o seguinte

Lema 4.2 Existem constantes $M>0, C>0, \delta^{\prime}>0$ e $\gamma \in(0,1)$ tais que

$$
\frac{1}{M} \leq|D(z, \lambda)| \leq M
$$




$$
\left|\psi_{\lambda}\left(z_{1}\right)-\psi_{\lambda}\left(z_{2}\right)\right| \leq C\left|z_{1}-z_{2}\right|^{\gamma}
$$

sempre que $z, z_{1}, z_{2} \in K_{0}$ e $\lambda \in \overline{\Delta^{N}}\left(\delta^{\prime}\right)$.

Demonstração. Primeiramente mostraremos que existem $\delta^{\prime}>0, C_{1}>0$ e $\gamma \in(0,1)$ tais que

$$
\left|D\left(z_{1}, \lambda\right)-D\left(z_{2}, \lambda\right)\right|=\left|g_{\lambda}^{\prime}\left(h_{\lambda} z_{1}\right)-g_{\lambda}^{\prime}\left(h_{\lambda} z_{2}\right)\right| \leq C_{1}\left|z_{1}-z_{2}\right|^{\gamma}
$$

para $z_{1}, z_{2} \in K_{0}$ e $|\lambda|_{0} \leq \delta^{\prime}$. Nossa intenção é aplicar o Teorema do Valor Médio à função $g_{\lambda}^{\prime}$. Na demonstração da Proposição 4.1 vimos que para qualquer aberto $V$ contendo $K_{0}$, existe um $\delta_{1}>0$ tal que

$$
K=\bigcup_{|\lambda|_{0} \leq \delta_{1}} h_{\lambda}\left(K_{0}\right)=h\left(\left\{|\lambda| \leq \delta_{1}\right\} \times K_{0}\right) \subset V .
$$

Recordando o $\lambda$-Lema, vemos que $K$, como imagem de um compato por meio de uma aplicação contínua, também é compacto. Logo, existe um $\eta>0$ de modo que, para quaisquer dois pontos $x$ e $y$ em $K$ com $|x-y| \leq \eta$, o segmento $[x, y]$ esteja contido em $V$. Da Proposição 4.1 concluímos que existem $\delta_{2}>0$ e $\gamma \in(0,1)$ tais que

$$
\left|h_{\lambda}\left(z_{1}\right)-h_{\lambda}\left(z_{2}\right)\right| \leq C\left|z_{1}-z_{2}\right|^{\gamma} \leq \eta
$$

sempre que $|\lambda| \leq \delta_{2}, z_{1}, z_{2} \in K_{0}$ e $\left|z_{1}-z_{2}\right| \leq \delta_{2}$.

Sendo $(\lambda, z) \mapsto g_{\lambda}(z)$ holomorfa, também o é $(\lambda, z) \mapsto g_{\lambda}^{\prime \prime}(z)$, como consequência do Teorema de Hartogs. Já os pólos da função racional $g_{0}^{\prime \prime}(z)$, que coincidem com os pólos de $g_{0}(z)$, estão fora de $K_{0}$ (uma vez que $K_{0}$ exclui o ponto no infinito e $g_{0}$ deixa invariante este conjunto); o que nos permite concluir que $g_{0}^{\prime \prime}(z)$ é limitada sobre $K_{0}$, digamos, por uma constante $M_{0}$. Como $K_{0}$ é compacto e $(\lambda, z) \mapsto g_{\lambda}^{\prime \prime}(z)$ é contínua, encontramos um $\delta_{3}>0$ e um aberto $W$ contendo $K_{0}$ de modo que

$$
\left|g_{\lambda}^{\prime \prime}(z)\right| \leq M_{0}+1=: M,
$$

para $z \in W$ e $|\lambda| \leq \delta_{3}$.

Agora voltamos ao início da argumentação e escolhemos $V=W$. Com isso, estamos prontos para provar (4.10). Escolhemos $\delta^{\prime}$ menor que quaisquer dos $\delta_{i}^{\prime} s$, e do Teorema do Valor Médio - juntamente com (4.11) - segue que

$$
\left|g_{\lambda}^{\prime}\left(h_{\lambda} z_{1}\right)-g_{\lambda}^{\prime}\left(h_{\lambda} z_{2}\right)\right| \leq M\left|h_{\lambda}\left(z_{1}\right)-h_{\lambda}\left(z_{2}\right)\right| \leq M C\left|z_{1}-z_{2}\right|^{\gamma}
$$

desde que tomemos $\left|z_{1}-z_{2}\right| \leq \boldsymbol{\delta}^{\prime}$. 
Finalmente, para obtermos (4.10) dividimos (4.12) por $\left|z_{1}-z_{2}\right|^{\gamma}$ e obtemos uma expressão $E\left(\lambda, z_{1}, z_{2}\right)$, a qual é contínua sobre o compacto $\left\{|\lambda| \leq \delta^{\prime}\right\} \times K_{0}^{2}$, exceto nos pontos onde $z_{1}=z_{2}$, nos quais $|E| \leq M C$. Logo, o supremo de $E$ é finito e daí concluímos (4.10). Em particular, $D(z, \lambda)$ é contínua sobre $\left\{|\lambda| \leq \delta^{\prime}\right\} \times K_{0}$ e (4.8) é trivialmente satisfeita, visto que $D(z, \lambda)$ nunca se anula, e também nunca vale $\infty$. A desigualdade (4.9) segue de (4.8) e (4.10) observando-se que $y=\log x$ é Lipschitziana sobre todo intervalo $\left[x_{0}, \infty\right), \operatorname{com} x_{0}>0$.

Lema 4.3 Seja $(X, \mathscr{B}, \mu)$ um espaço de probabilidade e $T$ uma transformação deste espaço nele mesmo. Se T preservar $\mu$, então

$$
\int f \circ T d \mu=\int f d \mu
$$

para toda função mensurável com respeito à $\mathscr{B}$. (Na igualdade acima, subentendese que se um dos lados não existir, ou for infinito, então o outro lado terá a mesma propriedade).

DemonstraÇão. Consultar qualquer referência introdutória de Teoria Ergódica por exemplo [49], página 25.

Com o Lema 4.2 sabemos agora que, para $\lambda$ suficientemente pequeno, todos os potenciais $\psi_{\lambda}$ estarão no mesmo espaço $C^{\gamma}\left(K_{0}\right)$, para algum $\gamma \in(0,1)$. Fixado $\lambda$ nessas condições, se $d=d(\lambda)$ e $\phi=\psi_{\lambda}$, então $P\left(d \phi ; g_{0}\right)=0$; e da Proposição 3.11 temos

$$
\frac{\partial P(d \phi)}{\partial d}=\int \phi d \mu
$$

onde $\mu$ é o estado de equilíbrio do sistema $\left(g_{0}, K_{0}\right)$ com potencial $d \phi$. A derivada acima é não-nula pois, sendo $\mu$ invariante por $g_{0}$ e $g_{\lambda}$ hiperbólica, do Lema anterior, de (2.1) e (2.3) segue que

$$
\begin{aligned}
n \int \phi d \mu & =\int S_{n} \phi(z) d \mu(z) \\
& =\int \sum_{i=0}^{n-1}-\log \left|g_{\lambda}^{\prime}\left(h_{\lambda} g_{0}^{i} z\right)\right| d \mu(z) \\
& =\int-\log \prod_{i=0}^{n-1}\left|g_{\lambda}^{\prime}\left(g_{\lambda}^{i} h_{\lambda} z\right)\right| d \mu(z) \\
& =\int-\log \left|\left(g_{\lambda}^{n}\right)^{\prime}\left(h_{\lambda} z\right)\right| d \mu(z) \\
& \leq-(n \log \alpha+\log C) ;
\end{aligned}
$$

e fazendo $n \rightarrow \infty$ encontramos 


$$
\int \phi d \mu \leq-\log \alpha<0
$$

Em particular, o princípio variacional nos diz que a entropia $h_{\mu}\left(g_{0}\right)$ do sistema $g_{0}$ com respeito ao estado de equilíbrio $\mu$ é estritamente positiva.

De volta a (4.13), sabendo-se que a média do potencial é não-nula, do Teorema da função implícita concluímos que todo $\phi_{0} \in C^{\gamma}\left(K_{0}, \mathbf{R}\right)$ possui uma vizinhança $V$ contida em $\mathscr{N}_{g_{0}}$ na qual $d$, dado implicitamente por $P(d \phi)$, se escreve como função analítica complexa $d=d(\phi)$ do potencial $\phi \in V$. Naturalmente, se provarmos que $\lambda \mapsto \psi_{\lambda} \in C^{\gamma}\left(K_{0}\right)$ é analítica real, então $d(\lambda)=d\left(\psi_{\lambda}\right)$ será uma função analítica real de $\lambda$. Deste modo

I. Para mostrar que $d(\lambda)$ é analítica real, é suficiente provar que $\lambda \mapsto \log |D(\cdot, \lambda)|$, como função de $\Delta^{N}(1)$ em $C^{\gamma}\left(K_{0}\right)$, é analítica real.

Um dos modos de se resolver este problema seria primeiro provar que, para cada $z$ fixado, $\lambda \mapsto \log |D(z, \lambda)|$ é analítica real, e depois tentar mostrar a afirmação em $(\mathbf{I})$. A primeira parte é relativamente fácil, mas o contexto natural da segunda é o caso complexo, como afirma o seguinte

Lema 4.4 Seja $K_{0}$ um subconjunto compacto e não-vazio do plano complexo e consideremos uma aplicação $L(z, \lambda)$, definida sobre $K_{0} \times \mathbf{C}^{d}$ e tomando valores em $\mathbf{C}$, tal que (i) $\lambda \mapsto L(z, \lambda)$ é holomorfa, para cada $z \in K_{0}$ fixado $e$

$$
\text { (ii) }\left|L\left(z_{1}, \lambda\right)-L\left(z_{2}, \lambda\right)\right| \leq C_{0}\left|z_{1}-z_{2}\right|^{\gamma}
$$

quaisquer que sejam $z_{1}, z_{2} \in K_{0}$, onde $C_{0}$ e $\gamma \in(0,1)$ são independentes dos pontos de $K_{0}$ e de $\lambda$. Nessas condições, $\lambda \mapsto L(\cdot, \lambda)$ é uma função holomorfa de $\Delta^{d}(1)$ em $C^{\gamma}\left(K_{0}\right)$.

Demonstração. Seja $\Phi(\lambda)=L(\cdot, \lambda)$, e escolhamos

$$
0<\varepsilon_{0}<\varepsilon_{1}<\varepsilon_{2}<1
$$

Existe uma constante positiva $r>0$, dependendo apenas das escolhas feitas acima, tal que $\lambda+w \mu \in \Delta^{d}\left(\varepsilon_{2}\right)$ sempre que $\lambda \in \Delta^{d}\left(\varepsilon_{1}\right), \mu \in \mathbf{C}^{d},|\mu|_{0} \leq 1, w \in \mathbf{C}$ e $|w| \leq r$. Das hipóteses (i) e (ii) tiramos que o supremo $C_{1}$ de $|L(z, \lambda)|$ sobre $K_{0} \times \overline{\Delta^{d}}\left(\varepsilon_{2}\right)$ é finito. De (1.37) segue-se que

$$
\left|\frac{\partial L(z, \lambda)}{\partial \lambda} \mu\right|=\left|\frac{1}{2 \pi i} \int_{|w|=r} \frac{L(z, \lambda+w \mu)}{w^{2}} d w\right| \leq \frac{C_{1}}{2 \pi}\left|\int_{|w|=r} \frac{1}{w^{2}}\right| d w=: C_{2} .
$$

Dados $z_{1}, z_{2} \in K_{0}$, colocamos

$$
F(\lambda)=\frac{L\left(z_{1}, \lambda\right)-L\left(z_{2}, \lambda\right)}{\left|z_{1}-z_{2}\right|^{\gamma}}
$$

Por (4.15), 


$$
\left|F\left(\lambda_{1}\right)-F\left(\lambda_{2}\right)\right| \leq C_{2}\left|\lambda_{1}-\lambda_{2}\right|,
$$

sempre que $\lambda_{1}, \lambda_{2} \in \overline{\Delta^{d}}\left(\varepsilon_{0}\right)$ estiverem uniformemente próximos um do outro (aqui usamos o Teorema do Valor Médio e um argumento padrão envolvendo propriedades de compactos que estão contidos no interior de um outro conjunto, como no Lema 4.2). Logo,

$$
\mathrm{C}_{\mathrm{H}}\left(\Phi\left(\lambda_{1}\right)-\Phi\left(\lambda_{2}\right)\right) \leq C_{2}\left|\lambda_{1}-\lambda_{2}\right|
$$

$\mathrm{e}$

$$
\left\|\Phi\left(\lambda_{1}\right)-\Phi\left(\lambda_{2}\right)\right\|_{\infty} \leq C_{2}\left|\lambda_{1}-\lambda_{2}\right|
$$

de onde segue que $\Phi(\lambda)$ é Lispschitz na variável $\lambda$, desde que tomemos $\lambda \in \Delta^{d}\left(\varepsilon_{0}\right)$. Completaremos a demonstração usando o Lema de Osgood, já que acabamos de mostrar que $\Phi$ é contínua. Escrevemos $\lambda$ na forma $\left(\lambda_{1}, \cdots, \lambda_{d}\right)$ e de (i) concluímos que a integral de $L(z, \lambda) d \lambda_{i}$ sobre qualquer curva fechada $\gamma$ contida na $i$-ésima coordenada vale zero. Por outro lado, a integral de $\Phi(\lambda) d \lambda_{i}$ sobre a mesma curva também existe, já que esta função é contínua. Com propriedades elementares de aproximação por somas de Riemann, vemos que

$$
\left(\int_{\gamma} \Phi(\lambda) d \lambda_{i}\right)(z)=\left(\int_{\gamma} L(\cdot, \lambda) d \lambda_{i}\right)(z)=\int_{\gamma} L(z, \lambda) d \lambda_{i}=0 .
$$

Do Teorema de Cauchy segue que $\Phi(\lambda)$ é holomorfa em cada variável separadamente, e portanto, deve ser holomorfa em $\lambda$.

Para podermos aplicar o resultado anterior na resolução de (I), vamos precisar de um Lema de extensão de funções reais para complexas. O próximo resultado nos foi apresentado por Juan Rivera-Letelier ([42]). (Fizemos algumas modificações no enunciado original).

Lema 4.5 (Extensão) Seja $D(z)$ uma função holomorfa definida no polidisco unitário $\Delta^{d}(1)$, tomando valores em $\mathbf{C}$, com

$$
\frac{1}{R} \leq|D(z)| \leq R
$$

para alguma constante $R>0$. Então existe uma única função holomorfa $\ell(z, w)$ definida para ze wem $\Delta^{d}(1 / 6)$, tomando valores em $\mathbf{C}$, tal que

$$
\ell(x, y)=\log |D(x+i y)|
$$

quando $x, y \in \Delta^{d}(1 / 6)$ forem reais. A função $\ell$ é não-nula em todo ponto de seu domínio, e para cada $\varepsilon$ no intervalo $(0,1 / 6)$, existe uma constante $C_{\varepsilon}$ dependendo somente de $\varepsilon$ e d tal que

$$
|\ell(z, w)-\ell(0,0)| \leq C_{\varepsilon}|\log R|
$$

sempre que z e w estiverem no polidisco $\Delta^{d}(\varepsilon)$. 
DEMONSTRAÇÃO. Usaremos o seguinte resultado:

(a) Seja $f(z)$ uma função holomorfa definida num polidisco ${ }^{7} \Delta^{N}(a, \varepsilon)$ de $\mathbf{C}^{N}$. Se $f(z) \neq 0$, para todo $z \in \Delta^{N}(a, \varepsilon)$, então existe um ramo analítico definido em $\Delta^{N}(a, \varepsilon)$ para a função $\log f(z)$.

Uma demonstração para o caso escalar pode ser encontrada em [1], pág 143. (Veja também a pág. 106 da mesma referência). Convidamos leitor a tentar adaptar a prova para o caso vetorial usando métodos de integração em várias variáveis (ver a prova do Lema de Osgood).

Usando este fato preliminar, definimos $\ell_{0}(z)$, para $z \in \Delta^{d}(1)$, como sendo um ramo analítico da função $\log D(z)$. E tendo em vista que a parte real de $\ell_{0}(z)$ coincide com $\log |D(z)|$, o que faremos é usar a representação em série de potências

$$
\ell_{0}(z)=\sum_{v} a_{v} z^{v}
$$

cujo raio de convergência uniforme é 1 (pela Proposição 1.17), e em seguida tomar $\ell(z, w)$ como sendo a complexificação formal da série de potências em variáveis reais

$$
\sum_{v} \operatorname{Re} a_{v}(x+i y)^{v}
$$

de onde virá

$$
\ell(x, y)=\operatorname{Re} \ell_{0}(x+i y)=\log |D(x+i y)| .
$$

Obviamente, existem detalhes que devemos especificar para que tenhamos as estimativas desejadas. Um polinômio homogêneo de grau $m$ nas variáveis $z_{1}, \ldots, z_{k}$ é qualquer soma de produtos da forma $\alpha z_{1}^{v_{1}} z_{2}^{v_{2}} \cdots z_{k}^{v_{k}}=\alpha z^{v}$, onde $v=\left(v_{i}\right)$ é um multíndice de norma $m$ e $\alpha \in \mathbf{C}$. (Essa noção inclui $x^{5} y+x^{3} y z$, que neste exemplo é um polinômio homogêneo de grau 5, mas exclui $x^{3}+x y^{3}$ ). Com isso, definimos

$$
P_{v}(x, y)=\operatorname{Re} a_{v}(x+i y)^{v}=\operatorname{Re}\left(\alpha_{v}+i \beta_{v}\right)\left(x_{1}+i y_{1}\right)^{v_{1}} \cdots\left(x_{d}+i y_{d}\right)^{v_{d}},
$$

onde $\alpha_{v}$ e $\beta_{v}$ são as partes real e imaginária de $a_{v}$. Este é um polinômio homogêneo nas variáveis $x_{1}, \ldots, x_{d}, y_{1}, \ldots, y_{d}$. Na expressão formal deste polinômio, complexificamos suas variáveis de $x_{i}, y_{i}$ para $z_{i}, w_{i}$, de modo a termos $P_{v}(z, w)$ definido para $z, w \in \mathbf{C}^{d}$. Nosso objetivo será mostrar que se $|z| \leq \varepsilon$ e $|w| \leq \varepsilon$, onde $\varepsilon<1 / 6$, então

$$
\left|P_{v}(z, w)\right| \leq 12 \times 2^{d}(6 \varepsilon)^{|v|}|\log R|, v \neq 0 .
$$

(b) Consideremos os polinômios homogêneos $P_{m}(x, y)$ e $Q_{m}(x, y)$, definidos como sendo a parte real e imaginária de $(x+i y)^{m}$, respectivamente $(x, y \in \mathbf{R})$. Na expressão formal destes polinômios, complexificamos suas variáveis, de modo a termos $P_{m}(z, w)$

$$
{ }^{7} \Delta^{N}(a, \varepsilon)=\left\{z \in \mathbf{C}^{N}:\left|z_{i}-a_{i}\right|<\varepsilon\right\}
$$


e $Q_{m}(z, w)$ definidos para quaisquer $z, w \in \mathbf{C}$. Então afirmamos que $P_{m}$ e $Q_{m}$ possuem grau $m$, e que existe um número natural $m_{0}$, independente de qualquer outra variável em questão, tal que

$$
\left|P_{v}(z, w)\right| \leq(2 \varepsilon)^{m},\left|Q_{m}(z, w)\right| \leq(2 \varepsilon)^{m},
$$

sempre que $z, w \in \Delta^{1}(\varepsilon)$ e $m \geq m_{0}$.

De fato, usando indução calculamos a forma literal de cada $P_{m}$ e $Q_{m}$, mostrando que eles são polinômios homogêneos de grau $m$. Já as desigualdades podem ser provadas do seguinte modo: observamos que se $z \neq 0$, então $P_{m}(z, w)=z^{m} P_{m}(1, w / z)$. Além disso, existe um modo recursivo de se obter $P_{m}(1, z)$ a partir de $P_{m-1}(1, z)$, o que nos permite escrever

$$
P_{m}(1, z)=a_{m}^{(m)} z^{m}+\cdots+a_{1}^{(m)} z+a_{0}^{(m)},
$$

obtendo uma cota superior para cada termo e, por conseguinte, $\left|P_{m}(1, z)\right| \leq 2^{m}$, para todo $m$ suficientemente grande. Disso segue (b) para $P_{m}$. O caso de $Q_{m}$ é feito da mesma forma.

Um argumento indutivo envolvendo (b) e a decomposição (4.18) nos leva a concluir que existe $k_{0}$, independente de qualquer outra variável, tal que

$$
\left|P_{v}(z, w)\right| \leq 2^{d}\left|a_{v}\right|(2 \varepsilon)^{v},
$$

sempre que $|v| \geq k_{0}$ e $z, w \in \Delta^{d}(\varepsilon)$. Agora procuremos estimar $\left|a_{v}\right|$.

Sabemos que a imagem de

$$
\ell_{0}(z)=\log |D(z)|+i \operatorname{Im} \ell_{0}(z)
$$

está contida no semiplano $\operatorname{Re} z<\log R$. Logo, $f(z)=\ell_{0}(z)-\log R$ verifica as condições do Corolário 1.2 (em cada variável separadamente). Disso segue que se $z$ estiver no polidisco $\Delta^{d}(1 / 3)$, então

$$
\begin{aligned}
\left|\ell_{0}(z)-\ell_{0}(0)\right| & =|f(z)-f(0)| \leq|z||f(z)+\overline{f(0)}| \\
& =\frac{1}{3}|\log R-\log | D(z)||+\frac{1}{3}|\log R-\log | D(0)||+\frac{1}{3}\left|\operatorname{Im}\left(\ell_{0}(z)-\ell_{0}(0)\right)\right| \\
& \leq \frac{2}{3}\left|\log R^{2}\right|+\frac{1}{3}\left|\operatorname{Im}\left(\ell_{0}(z)-\ell_{0}(0)\right)\right| .
\end{aligned}
$$

Usando a desigualdade $|\cdot|_{+} \leq 2|\cdot|_{e}$ entre a norma da soma e a norma euclidiana e obtemos de (4.21) que

$$
\left|\operatorname{Re}\left(\ell_{0}(z)-\ell_{0}(0)\right)\right|+\left|\operatorname{Im}\left(\ell_{0}(z)-\ell_{0}(0)\right)\right| \leq \frac{4}{3}\left|\log R^{2}\right|+\frac{2}{3}\left|\operatorname{Im}\left(\ell_{0}(z)-\ell_{0}(0)\right)\right| ;
$$


de onde vem

$$
\left|\operatorname{Re}\left(\ell_{0}(z)-\ell_{0}(0)\right)\right|+\left|\operatorname{Im}\left(\ell_{0}(z)-\ell_{0}(0)\right)\right| \leq 4\left|\log R^{2}\right|
$$

$\mathrm{e}$

$$
\left|\ell_{0}(z)-\ell_{0}(0)\right| \leq \sqrt{2} \times 4\left|\log R^{2}\right| \leq 12|\log R| .
$$

E como $\ell_{0}(z)-\ell_{0}(0)$ é exatamente a soma $\operatorname{dos} a_{v} z^{v}$ para $v \neq 0$, de (1.45) concluímos que

$$
\left|a_{v}\right| \leq 12|\log R| 3^{|v|} .
$$

Finalmente, observamos que cada $P_{v}$ é um polinômio homogêneo, e que em vista do que acabamos de provar, a série de potências

$$
\ell(z, w)=\operatorname{Re} \ell_{0}(0)+\sum_{v \neq 0} P_{v}(z, w)
$$

converge uniformemente sobre $\left(\Delta^{d}(\varepsilon)\right)^{2}$, desde que tomemos $\varepsilon<1 / 6$. Segue-se que $\ell(z, w)$ é uma função analítica nas condições do enunciado, com $C_{\varepsilon}$ dependendo da soma da série numérica convergente que domina (4.23). Deixamos a cargo do leitor verificar a afirmação de unicidade, bem como que $\ell$ nunca se anula.

Agora estamos prontos para demonstrar a afirmação contida em (I) e, consequentemente, que $d(\lambda)$ é analítica real.

No Lema 4.2, podemos trocar $\Delta^{N}\left(\delta^{\prime}\right)$ por $\Delta^{N}(1)$ e supor que as propriedades lá descritas valem para qualquer $\lambda$ no polidisco unitário. (Existe uma equivalência conforme entre estas duas regiões). Assim, do Lema da Extensão concluímos que existe uma função

$$
L(z, \lambda): K_{0} \times\left(\Delta^{N}(1 / 6)\right)^{2} \rightarrow \mathbf{C}
$$

tal que

$$
L(z ; \xi, \eta)=\log |D(z, \xi+i \eta)|,
$$

sempre que $\xi, \eta \in \mathbf{R}^{N}, \xi, \eta \in\left(\Delta^{N}(1 / 6)\right)^{2}$; com

(1) $L(z, \lambda)$ sendo uma função holomorfa na variável $\lambda \in\left(\Delta^{N}(1 / 6)\right)^{2}, \forall z \in K_{0}$.

Novamente, vamos reparametrizar a função acima, e supor que $\lambda$ varia em $\left(\Delta^{N}(1)\right)^{2}$. Dados $z_{1}, z_{2}$ em $K_{0}$, definimos

$$
D(\lambda)=\frac{D\left(z_{1}, \lambda\right)}{D\left(z_{2}, \lambda\right)}
$$

Pelo Lema 4.2,

$$
|D(\lambda)| \leq \exp C\left|z_{1}-z_{2}\right|^{\gamma} \text { e }\left|D(\lambda)^{-1}\right| \leq \exp C\left|z_{1}-z_{2}\right|^{\gamma} .
$$


Seja $L(\lambda)$ a função dada pelo Lema da extensão. Com (4.17), é fácil ver que existe uma constante $C_{0}$ independente de $z_{1}$ e $z_{2}$ tal que $|L(\lambda)| \leq C_{0}\left|z_{1}-z_{2}\right|^{\gamma}$. E pela unicidade da extensão, $L=L\left(z_{1}, \cdot\right)-L\left(z_{2}, \cdot\right)$. Disso segue que

(2) Para todo $\lambda \in\left(\Delta^{N}(1)\right)^{2},\left|L\left(z_{1}, \lambda\right)-L\left(z_{2}, \lambda\right)\right| \leq C_{0}\left|z_{1}-z_{2}\right|^{\gamma}$.

As condições (1) e (2), juntamente com o Lema 4.4, fazem com que $\lambda \mapsto L(\cdot, \lambda)$ seja analítica sobre $\left(\Delta^{N}(1)\right)^{2}$. Em particular, de $(\mathbf{I})$ e (4.24) concluímos que $d(\lambda) e ́$ analítica real. 


\section{Medidas de Haar}

Estudaremos agora um tipo especial de medida de Radon, as medidas de Haar. Tais medidas estão definidas num grupo topológico $G$, e preservam a estrutura algébrica deste grupo, num sentido que deixaremos claro logo mais.

Um grupo topológico é um grupo $(G, \cdot)$ munido de uma topologia que torna $G$ Hausdorff e as operações $(x, y) \mapsto x y$ e $x \mapsto x^{-1}$ contínuas. O espaço Euclidiano $\left(\mathbb{R}^{n},+\right)$ e, mais geralmente, os espaços vetoriais topológicos, são exemplos de grupos topológicos. Para $x \in G$ e $A, B \subset G$, escreveremos

$$
\begin{gathered}
x A=\{x y: y \in A\}, A x=\{y x: y \in A\}, \\
A^{-1}=\left\{x^{-1}: x \in A\right\} \text { e } A B=\{y z: y \in A, z \in B\} .
\end{gathered}
$$

Da continuidade das operações do grupo seguem propriedades bem interessantes. A primeira delas é que se $U$ for um aberto de $G$ então também serão $U x$ e $x U$; deste modo, à cada ponto $x \in G$ estão associados dois homeomorfismos $y \mapsto y x$ e $y \mapsto x y$. Um conjunto $A \subset G$ será chamado de simétrico quando for igual ao seu inverso, isto é, $A=A^{-1}$. O inverso de um aberto também é aberto e, em vista disso, podemos afirmar que para qualquer vizinhança $V$ do elemento neutro e existe uma outra vizinhança simétrica $U$ de e tal que $U \subset V$. Podemos, ainda, tomar a vizinhança simétrica $U$ de modo que $U U \subset V$. Para ver isso, consideremos a função $\varphi(x, y)=x y$; a imagem inversa $\varphi^{-1}(U)$ é uma vizinhaça de $(e, e)$, para a qual existem abertos $A, B$ de $G$ tais que $(e, e) \in A \times B \subset \varphi^{-1}(U)$. Tomamos $V_{1}=A \cap B$ e depois $V=V_{1} \cap V_{1}^{-1}$. Se $\left\{x_{\alpha}\right\}$ e $\left\{y_{\beta}\right\}$ forem nets de um subgrupo $H<G$ e $x_{\alpha} \rightarrow x, y_{\beta} \rightarrow y$, então $x_{\alpha} y_{\beta}^{-1} \rightarrow x y^{-1}$, pela continuidade das operações do grupo. Concluímos daí que ofecho $\bar{H}$ de qualquer subgrupo $H$ de $G$ também é um sugbrupo de $G$. Seguindo o mesmo argumento também verifica-se que se $H$ for normal em $G$, então seu fecho também o será. Todo grupo $G$ decompõe-se como reunião disjuntas das classes laterais $x H$ de um subgrupo qualquer $H$, não necessariamente normal. Teremos $H=x H$ se, e somente se, $x \in H$; de modo que

$$
H=G \backslash \bigcup_{x \in H^{c}} x H .
$$


Segue daí que se $H$ for aberto, então $H$ também será fechado. Assim, todo subgrupo aberto de $G$ também é fechado. Também é fácil ver que o produto $K_{1} K_{2}$ de quaisquer compactos $K_{1}$ e $K_{2}$ deve ser compacto, pois $\varphi\left(K_{1} \times K_{2}\right)=K_{1} K_{2}$, onde $\varphi(x, y)=x y$.

De agora em diante iremos supor que $(G, \cdot)$ é um grupo topológico localmente compacto. São nestes grupos que definiremos as medidas de Haar; mais tarde mostraremos que todo grupo topológico localmente compacto possui uma medida de Haar.

Uma medida de Borel $\mu$ sobre $G$ será chamada de invariante à esquerda se $\mu(x E)=\mu(E)$, para todo Boreliano $E$; do mesmo modo, se $\mu(E)=\mu(E x)$, então diremos que $\mu$ é invariante à direita. As medidas de Radon não nulas $\mu$ sobre $G$ que forem invariantes à esquerda, ou à direita, serão chamdas de medidas de Haar. Quando quisermos especificar que a medida de Haar $\mu$ é invariante à esquerda, ou que $\mu$ é invariante à direita, diremos simplesmente que $\mu$ é Haar à esquerda, ou que $\mu$ é Haar à direita. Usando o Teorema da Representação de Riesz, sabemos que à cada medida de Haar $\mu$ corresponde um funcional linear positivo $I_{\mu}$ sobre $C_{c}(G)$. Seria interessante, sob este ponto de vista, dizer quando um funcional $I$ sobre $C_{c}(G)$ é invariante à esquerda, ou à direita, e tentar caracterizar os funcionais positivos que correspondem a medidas de Haar.

Se $y \in G$, então definimos os operadores de transalação à esquerda $L_{y}$ e à direita $R_{y}$ sobre $C_{c}(G)$ por

$$
L_{y} f(x)=f\left(y^{-1} x\right), \quad R_{y} f(x)=f(x y) .
$$

É imediato verificar que $L_{y z}=L_{z} L_{y}$, bem como $R_{y z}=R_{y} R_{z}$, e que $L_{y} f$ e $R_{y} f$ serão elementos de $C_{c}(G)$ sermpre que $f \in C_{c}(G)$. Diremos então que um funcional linear (não necessariamente limitado) $I$ sobre $C_{c}(G)$ é invariante à esquerda se $I\left(L_{y} f\right)=I(f), f \in$ $C_{c}(G)$; e invariante à direita se $I\left(R_{y} f\right)=I(f)$, para todo $f \in C_{c}(G)$. A seguir listamos algumas propriedades sobre medidas de Haar. Utilizaremos a notação $C_{c}^{+}$para indicar o conjunto das $f \in C_{c}(G)$ tais que $f \geq 0$ e $\|f\|_{u}>0$, onde $\|f\|_{u}=\sup \{|f(x)|: x \in G\}$ é a norma uniforme de $f$. Mostraremos, entre outras coisas, que uma medida de Radon $\mu$ sobre $G$ será Haar à esquerda se, e somente se, o funcional associado $I_{\mu}(f)=\int f d \mu$ for invariante à esquerda quando restrito à $C_{c}^{+}$(é fácil verificar que $L_{y}\left(C_{c}^{+}\right) \subset C_{c}^{+}$).

Teorema A.1 Seja $\mu$ uma medida de Radon sobre um grupo topológico localmente compacto G. Então

(1) $\mu$ será Haar à esquerda se, e somente se, a medida $\check{\mu}$ dada por $\breve{\mu}(E)=$ $\mu\left(E^{-1}\right)$ for Haar à direita;

(2) $\mu$ será Haar à esquerda se, e somente se, $\int f d \mu=\int L_{y} f d \mu$ para qualquer $f \in C_{c}^{+}$e $y \in G$;

(3) Se $\mu$ for Haar à esquerda, então $\mu(U)>0$ para todo aberto $U \neq \emptyset$, e $\int f d \mu>0$ para toda $f \in C_{c}^{+}$;

(4) Se $\mu$ for Haar à esquerda, então $\mu(G)<\infty$ se, e somente se $G$ for compacto.

Nosso principal objetivo é provar dois resultados. O primeiro é sobre a existência de medidas de Haar em grupos topológicos localmente compactos; o segundo é sobre a 
unicidade de tais medidas, no sentido de que se $\mu$ e $v$ forem medidas de Haar sobre um mesmo grupo topológico $G$, então existirá uma constante $c>0$ tal que $\mu=c v$ (o mesmo vale para medidas de Haar à direita). Em vista do Teorema A.1(1) será suficiente estudar o caso em que as medidas são Haar à esquerda.

DEMONSTRAÇÃO DO TEOREMA A.1 - (1) Basta verificar que as condições para que $\check{\mu}$ seja medida, com $\breve{\mu}(E)=\breve{\mu}(E x)$, para todo Boreliano $E$ e todo $x \in G$. (2) Valendo a igualdade para integrais, consideramos o caso particular em que $f=\chi_{K}, K \neq \emptyset$ compacto, e concluímos a partir daí que $\mu(K)=\mu(y K)$, para todo $y \in G$. Como $\mu$ é Radon, podemos aproximar a medida de cada aberto $U$ pelas medidas de compactos $K \subset U$ e, por sua vez, a medida de cada Boreliano $E$ pode ser aproximada pelas medidas de abertos $U \supset E$. Daí concluímos que $\mu(E)=\mu(y E)$, para todo Boreliano $E$ e todo $y \in G$. Reciprocamente, se $\mu$ for Haar à esquerda sobre $G$, então a igualdade entre as integrais será imediata quando $f \in C_{c}^{+}$for simples. Como o conjunto de tais funções é denso em $C_{c}^{+} \subset \mathscr{L}^{1}(\mu)$, concluímos que a igualdade em (2) vale para toda função $f \in C_{c}^{+}$. (3) Como $\mu \neq 0$, temos $\mu(G)>0$; e, pela regularidade de $\mu$, existe um compacto $K \subset G$ tal que $\mu(K)>0$. Para qualquer aberto não vazio $U$ existe um número finito de classes laterais $x_{1} U, x_{2} U, \ldots, x_{n} U$ que cobrem K. Como $\mu$ é Haar à esquerda, $\mu\left(x_{j} U\right)=\mu(U)$ e segue daí que $\Sigma_{1}^{n} \mu(U) \geq \mu(K)>0$; $\log , \mu(U)>0$. (4) Se $G$ for compacto, então $\mu(G)<\infty$, pois $\mu$ é Radon. Reciprocamente, mostremos que se $G$ não for compacto, então $\mu(G)=\infty$. De fato, seja $V$ uma vizinhança compacta qualquer de $e$. Como $G$ não é compacto, podemos encontrar uma sequência $\left\{x_{j}\right\}_{1}^{\infty} \subset G$ tal que

$$
x_{n} \in\left(\bigcup_{j=1}^{n-1} x_{j} V\right)^{c}, n \geq 2,
$$

já que $G$ não pode ser coberto por qualquer reunião finita de compactos $x_{j} V$. Se $U$ for uma vizinhança simétrica e contida em $V$, então os conjuntos $x_{j} U$ serão disjuntos dois a dois, pois se $x_{n} U \cap x_{m} U \neq \emptyset$, com, digamos, $m>n$, então $x_{m} \in x_{n} U U \subset x_{n} V$, o que vai contra $(A .1)$. Como $\mu(U)>0$ e $\mu\left(x_{j} U\right)=\mu(U)$, segue então que $\mu(G)=\infty$.

Agora mostraremos a existência de medidas de Haar à esquerda num grupo topológico localmente compacto. A parte (2) do Teorema A.1 nos indica um caminho: basta constuir um funcional linear positivo $I$ sobre $C_{c}(G)$, ao qual corresponderá uma medida de Radon $\mu$, que será Haar à esquerda se, e somente se, $I$ for invariante à esquerda sobre $C_{c}^{+}$. Buscando uma motivação sobre como construir tal funcional, consideremos inicialmente uma medida de Haar à esquerda $\mu$ sobre $G$, e procuremos relacioná-la, heuristicamente, com um possível funcional sobre $C_{c}^{+}$. Se $V \neq \emptyset$ for uma vizinhança qualquer de $e$, sabemos que a medida de qualquer reunião reunião disjunta de classes $x V, x \in A \subset G$, é igual à $\operatorname{Card}(A) \mu(A)$, com a convenção de que $\operatorname{Card}(A)=\infty$ se $A$ for infinito. De um modo evidentemente vago, podemos inferir que à medida que $V$ "encolher" para $e$, os valores correspondentes de 


$$
(E: V):=\inf \left\{\operatorname{Card}(A): \bigcup_{x \in A} x V \supset E\right\}
$$

serão uma boa aproximação de $\mu(E)$, para todo Boreliano $E$. Fixemos um aberto $E_{0}$ não vazio, cujo fecho seja compacto em $G$. Pelo Teorema A.1 $(3,4)$ sabemos que $0<$ $\mu\left(E_{0}\right)<\infty$ e, pelo que acabamos de observar, a expressão

$$
\mu_{V}(E):=\frac{(E: V)}{\left(E_{0}: V\right)}
$$

define uma função da $\sigma$-álgebra de Borel de $G$ em $[0, \infty]$ tal que $\mu_{V} \approx c \mu$, para alguma constante $c>0$. A vantagem de se trabalhar com as aproximações $\mu_{V}$ é que elas dependem somente de $V, E_{0}$ e da estrutura algébrica de $G$. Para transportarmos este fato para o estudo de funcionais positivos sobre $C_{c}^{+}$, busquemos expressar $(E: V) \mathrm{em}$ termos de funções características. Para $f$ e $\phi$ em $C_{c}^{+}$, definimos

$$
(f: \phi)=\inf \left\{\sum_{j=1}^{n} c_{j}: \exists x_{1}, \ldots x_{n} \in G \text { t.q. } f \leq \sum c_{j} L_{x_{j}} \phi\right\} .
$$

O número $(f: \phi)$ é chamado de número de cobertura de Haar de $f$ com respeito a $\phi$ e sempre existe, pois o conjunto acima é não vazio. De fato,

$$
U:=\left\{x: \phi(x)>\frac{1}{2}\|\phi\|_{u}\right\}
$$

é aberto, não vazio, e, portanto, existe um número finito de classes laterais $x_{1} U, \ldots x_{n} U$ que cobrem o suporte $K$ de $f$. Assim, para todo $x \in K$ existe um $x_{j}$ tal que $x_{j}^{-1} x \in U$ e, portanto, $2 \phi\left(x_{j}^{-1} x\right)>\|\phi\|_{u}$; em particular podemos escrever

$$
2 \sum_{j=1}^{n} L_{x_{j}} \phi(x)=2 \sum_{j=1}^{n} \phi\left(x_{j}^{-1} x\right)>\|\phi\|,
$$

de onde se conclui que

$$
f(x) \leq\|f\|_{u} \frac{\|\phi\|_{u}}{\|\phi\|_{u}}<\frac{2\|f\|_{u}}{\|\phi\|_{u}} \sum_{j=1}^{n} L_{x_{j}} \phi(x) .
$$

O número $(f: \phi)$ é um equivalente de $(E: V)$ para funções em $C_{c}^{+}$. De fato, se $\chi_{E} \in C_{c}^{+}$ e $V$ for tal que seu fecho é compacto, então podemos escrever

$$
(E: V)=\inf \left\{\sum_{x \in A} 1: \bigcup_{x \in A} x V \supset E\right\}
$$




$$
=\sup \left\{\sum_{x \in A} 1: \chi_{E} \leq \sum_{x \in A} L_{x} \chi_{V}\right\}=\inf \left\{\sum_{j=1}^{n} 1: \chi_{E} \leq \sum_{j=1}^{n} L_{j=1}^{n} L_{x_{j}} \chi_{V}\right\},
$$

onde a última igualdade é justificada pelo fato de que o fecho de $E$ é compacto e pode sempre ser coberto por um número finito de classes laterais $x_{j} V$. Apesar de no último conjunto aparecer uma soma de 1's em vez de constantes arbitrárias $c_{j}$, como em $(f: \phi)$, não há motivo algum para restringirmos os valores de $c_{j}$ na definição de $(f: \phi)$, uma vez que nossa discussão envolvendo $(E: V)$ é apenas heurística. Como

$$
(f: \phi) \geq\|f\|_{u} /\|\phi\|_{u}>0
$$

é sempre não nulo, uma vez fixado $f_{0} \in C_{c}^{+}$, podemos considerar a expressão

$$
I_{\phi}(f)=\frac{(f: \phi)}{(f: \phi)}
$$

que define um funcional positivo sobre $C_{c}^{+}$. Embora não possamos afirmar nada sobre a linearidade deste funcional, sabemos que ele será "quase linear", quando o suporte de $\phi$ for bem pequeno (ver Lema A.2). O primeiro resultado neste sentido é o seguinte

Lema A.1 Suponhamos que $f, g, \phi \in C_{c}^{+}$. Então

(1) $(f: \phi)=\left(L_{x} f: \phi\right)$, para todo $x \in G$;

(2) $(c f: \phi)=c(f: \phi)$, para qualquer $c>0$;

(3) $(f+g: \phi) \leq(f: \phi)+(f: \phi)$;

(4) $(f: \phi) \leq(f: g)(g: \phi)$.

DemonstraÇÃo. - (1) Segue de

$$
f \leq \sum_{j=1}^{n} c_{j} L_{x_{j}} \phi \Longleftrightarrow L_{x} f \leq \sum_{j=1}^{n} c_{j} L_{x x_{j}} \phi .
$$

(2) Imediada. (3) Dado $\varepsilon>0$, sejam $x_{1}, \ldots x_{n}, x_{n+1}, \ldots x_{m}$ tais que

$$
f \leq \sum_{j=1}^{n} c_{j} L_{x_{j}} \phi, g \leq \sum_{j=n+1}^{m} c_{j} L_{x_{j}} \phi
$$

e

$$
\sum_{j=1}^{n} c_{j}<(f: \phi)+\frac{\varepsilon}{2}, \quad \sum_{j=n+1}^{m} c_{j}<(g: \phi)+\frac{\varepsilon}{2} .
$$

Então

$$
f+g \leq \sum_{j=1}^{m} c_{j} L_{x_{j}} \phi
$$

$$
\sum_{j=1}^{m} c_{j} \leq(f: \phi)+(g: \phi)+\varepsilon,
$$


de onde segue que $(f+g: \phi) \leq(f: \phi)+(g: \phi)+\varepsilon$ para $\varepsilon>0$ arbitrário.

(4) $\mathrm{Se}$

$$
f \leq \sum c_{j} L_{x_{j}} g \quad \text { e } \quad g \leq \sum d_{k} L_{x_{k}} \phi
$$

com

$$
\sum c_{j} \leq(f: g)+\varepsilon \text { e } \sum d_{k} \leq(g: \phi)+\varepsilon
$$

então

$$
f \leq \sum_{j} c_{j}\left(\sum d_{k} L_{x_{j} x_{k}} \phi\right)=\sum_{j, k} c_{j} d_{k} L_{x_{j} x_{k}} \phi
$$

e, portanto,

$$
(f: \phi) \leq \sum_{j, k} c_{j} d_{k}=\left(\sum c_{j}\right)\left(\sum d_{k}\right) \leq[(f: g)+\varepsilon][(g: \phi)+\varepsilon] .
$$

Fazendo $\varepsilon \rightarrow 0$ segue (4).

Do Lema anterior concluímos que o funcional $I_{\phi}$ dado pela equação (A.2) é subaditivo [Lema A.1(3)], invariante à esquerda, i.é., $I_{\phi}\left(L_{x} f\right)=I_{\phi}(f)$ [Lema A.1(1)] e satisfaz $\left(f_{0}: f\right)^{-1} \leq I_{\phi}(f) \leq\left(f: f_{0}\right)$, [Lema A.1(4)].

Lema A.2 Dado $\varepsilon>0$ existe uma vizinhaça $V$ de e tal que

$$
I_{\phi}\left(f_{1}\right)+I_{\phi}\left(f_{2}\right) \leq I_{\phi}\left(f_{1}+f_{2}\right)+\varepsilon,
$$

sempre que o suporte de $\phi$ estiver contido em $V$.

A demonstração deste Lema requer o seguinte resultado sobre continuidade uniforme de uma função $f$ em $C_{c}(G)$.

Proposição A.1 Se $f \in C_{c}(G)$, então, para todo $\varepsilon>0$, existe uma vizinhança $V$ de $e$ tal que $\left\|L_{y} f-f\right\|_{u}<\varepsilon$ e $\left\|R_{y} f-f\right\|<\varepsilon$, sempre que $y \in V$.

DEMONSTRAÇÃo. - Mostraremos somente a primeira desigualdade; a segunda é provada do mesmo modo. Sejam $\varepsilon>0$ e $K$ o suporte de $f$. Como $f$ é contínua, para todo $x \in K$ existe uma vizinhança $U_{x}$ de $e$ em $G$ tal que

$$
\left|f\left(y^{-1} x\right)-f(x)\right|<\frac{\varepsilon}{2}, y \in U_{x}
$$

ou, de outro modo, $|f(z)-f(x)|<\varepsilon / 2$, para todo $z \in G$ tal que $x z^{-1} \in U_{x}$. Para cada $x \in K$ escolhemos uma vizinhança simétrica $V_{x}$ de $e$ tal que $V_{x} V_{x} \subset U_{x}$. A família $\left\{V_{x}^{-1} x\right\}_{x \in K}=\left\{V_{x} x\right\}_{x \in K}$ cobre o compacto $K$ e, portanto, existem $x_{1}, \ldots, x_{n}$ em $K$ tais que $\left\{V_{x_{j}} x_{j}\right\}_{1}^{n}$ ainda cobre $K$. Seja $V=\bigcap_{1}^{n} V_{x_{j}}$. Se $x \in K$ e $y \in V$, então existe $j$ tal que $x_{j} x^{-1} \in V_{x_{j}} \mathrm{e}$

$$
\left|f\left(y^{-1} x\right)-f(x)\right| \leq\left|f\left(y^{-1} x\right)-f\left(x_{j}\right)\right|+\left|f\left(x_{j}\right)-f(x)\right|<\frac{1}{2} \varepsilon+\frac{1}{2} \varepsilon=\varepsilon,
$$


pois $x_{j}\left(y^{-1} x\right)^{-1}=x_{j} x^{-1} y^{-1} \in V_{x_{j}} V_{x_{j}} \subset U_{x_{j}}$ e $x x_{j} \in V_{x_{j}}^{-1}=V_{x_{j}} \subset U_{x_{j}}$. Caso $x$ não esteja em $K$, teremos $f(x)=0$ e, portanto,

$$
\left|f\left(y^{-1} x\right)-f(x)\right|=\left|f\left(y^{-1} x\right)\right|<\varepsilon,
$$

pois se $y^{-1} x \in K$ então $f\left(y^{-1} x\right)=0$ e, caso contrário, $\left|f\left(y^{-1} x\right)\right|<\varepsilon$, pelo mesmo argumento que foi usado no caso em que $x \in K$.

Demonstração do Lema A. 2 - Sejam $f_{1}, f_{2} \in C_{c}^{+}, \varepsilon>0$ e coloquemos $h=f_{1}+$ $f_{2}+\delta g$, onde $\delta$ é um número não-negativo que fixaremos adiante, e $g$ é qualquer função de $C_{c}^{+}$que vale 1 no suporte de $f_{1}+f_{2}$. Com a convenção de que $0 / 0=0$, podemos definir $h_{i}=f_{i} / h(i=1,2)$, pois $f_{i}$ se anula em qualquer ponto onde $h$ se anula. As funções $h_{i}$ são contínuas, uma vez que o suporte de cada $f_{i}$ está contido no aberto $\{x: h(x) \neq 0\}$, onde cada $h_{i}$ é contínua - de fato, $h(x)>\delta$ sobre $\operatorname{supp}\left(f_{1}+f_{2}\right) \supset$ $\operatorname{supp}\left(f_{i}\right)$. Além disso, $h_{i} \in C_{c}^{+}$e, deste modo, existe uma vizinhança $V_{\delta}$ de $e$ tal que $\left|h_{i}(x)-h_{i}(y)\right|<\delta$ sempre que $y^{-1} x \in V_{\delta}$.

Agora mostraremos que se tomarmos $\delta>0$ suficientemente pequeno e $\operatorname{supp}(\phi) \subset$ $V_{\delta}$, então teremos

$$
I_{\phi}\left(f_{1}\right)+I_{\phi}\left(f_{2}\right) \leq I_{\phi}\left(f_{1}+f_{2}\right)+\varepsilon .
$$

Para tanto, suponhamos que $h \leq \sum_{1}^{n} c_{j} L_{x_{j}} \phi$, onde $\phi \in C_{c}^{+} \operatorname{e} \operatorname{supp}(\phi) \subset V$. Se $x_{j}^{-1} x \in$ $\operatorname{supp}(\phi)$, então $h_{i}(x)<h_{i}\left(x_{j}\right)+\delta$ e, caso contrário, $\phi\left(x_{j}^{-1} x\right)=0$; assim, podemos escrever

$$
f_{i}(x)=h(x) h_{i}(x) \leq \sum_{j=1}^{n} c_{j} \phi\left(x_{j}^{-1} x\right) h_{i}(x) \leq \sum_{j=1}^{n} c_{j} \phi\left(x_{j}^{-1} x\right)\left[h_{i}(x)+\delta\right],
$$

o que implica

$$
\left(f_{i}: \phi\right) \leq \sum_{j=1}^{n} c_{j}\left[h_{i}\left(x_{j}\right)+\delta\right]
$$

E como $h_{1}+h_{2} \leq 1$, segue-se que

$$
\left(f_{1}: \phi\right)+\left(f_{2}: \phi\right) \leq(1+2 \delta) \sum_{j=1}^{n} c_{j}
$$

Mas $\sum_{1}^{n} c_{j}$ pode ser feito arbitrariamente próximo de $(h: \phi)$, de modo que $\left(f_{1}: \phi\right)+$ $\left(f_{2}: \phi\right) \leq(1+2 \delta)(h: \phi)$ e, portanto,

$$
I_{\phi}\left(f_{1}\right)+I_{\phi}\left(f_{2}\right) \leq(1+2 \delta)\left[I_{\phi}\left(f_{1}+f_{2}\right)+\delta I_{\phi}(g)\right] .
$$

Para concluir o resultado, basta tomar $\delta>0$ de modo que

$$
2 \delta\left(f_{1}+f_{2}: f_{0}\right)+\delta(1+2 \delta)\left(g: f_{0}\right)<\varepsilon .
$$


Uma vez mostrado que os funcionais $I_{\phi}$ são aproximadamente aditivos - no sentido do Lema que acabamos de provar - estamos prontos para demonstrar o teorema de existência de medidas de Haar. A idéia de sua demonstração é encontrar um funcional $I: C_{c}(G) \rightarrow \mathbb{R}$, que esteja arbitrariamente próximo dos funcionais $I_{\phi}$, quando restrito à $C_{c}^{+}$. Usaremos o fato de cada $I_{\phi}$ ser invariante à esquerda, positivo e "quase linear" para mostrar que $I$ goza da mesma propriedade. Em vista do que já comentamos, ao funcional linear positivo e invariante à esquerda $I$ corresponderá uma medida de Haar à esquerda sobre $G$.

Teorema A.2 (Existência de medidas de Haar) Todo grupo topológico localmente compacto e Hausdorff possui medidas de Haar à esquerda e à direita.

DEMONSTRAÇÃO. - Como toda medida de Haar à esquerda induz naturalmente uma outra medida de Haar à direita, e vice-versa, basta demonstrar o caso à esquerda. Para $f \in C_{c}^{+}$, sabemos que os intervalos $X_{f}=\left[\left(f_{0}: f\right)^{-1},\left(f: f_{0}\right)\right]$ são não vazios e, pelo Teorema de Tychonoff, o espaço produto

$$
X=\prod_{f \in C_{c}^{+}} X_{f}
$$

é compacto e, além disso, Hausdorff. Pelo modo como formamos $X$, cada funcional $I_{\phi}$ dever ser um elemento de $X$. Para cada vizinhança $V$ de $e$, consideramos o conjunto

$$
K(V)=\overline{\left\{I_{\phi}: \operatorname{supp}(\phi) \subset V\right\}} .
$$

Qualquer interseção finita da família dos fechados $K(V)$ é não vazia, pois, de fato,

$$
\bigcap_{j=1}^{n} K\left(V_{j}\right)=K\left(\bigcap_{j=1}^{n} V_{j}\right) \neq \emptyset .
$$

Logo, existe um funcional $I \in X$ que está em todos os conjuntos $K(V)$. Em outras palavras, dados $f_{1}, \ldots, f_{n} \in C_{c}^{+}$e $\varepsilon>0$, sempre existe um $J \in K(V)$ de modo que $\left|J\left(f_{i}\right)-I\left(f_{i}\right)\right|<\varepsilon(i=1, \ldots, n)$; e se $I_{\phi} \in K(V)$ for suficientemente próximo de $J$, então teremos $\left|I_{\phi}\left(f_{i}\right)-I\left(f_{i}\right)\right|<\varepsilon$, para $i=1, \ldots, n$. Com o auxílio desta propriedade, dos Lemas A.1 e A.2, e um simples argumento em torno da desigualdade triangular, conseguimos provar que $I$ é invariante à esquerda sobre $C_{c}^{+}$e que, além disso, $I(a f+g)=a I(f)+I(g)$, quaisquer que sejam $a>0$ e $f, g \in C_{c}^{+}$. Extendemos $I$ a $C_{c}^{+}$colocando $I(0)=0$ e depois $I(f)=I\left(f^{+}\right)-I\left(f^{-}\right)$. Obtemos assim, um funcional linear positivo e invariante à esquerda sobre $C_{c}(G)$, como queríamos.

O teorema que acabamos de demonstrar é devido à HAAR (ver [21]). Nele utilizamos o Axioma da Escolha, que ficou implícito quando aplicamos o Teorema de Tychonoff para mostrar que $X=\prod_{f} X_{f}$ é compacto. Existe uma outra demonstração, que não usa este axioma - esta devida à $H$. Cartan, - que pode ser encontrada em [24], no parágrafo 15. O livro de Halmos sobre Teoria da Medida (ver [22]) contém uma 
exposição que, diferentemente da nossa, trabalha diretamente com os números $(E: V)$ em vez dos funcionais $I_{\phi}$.

Agora mostraremos a unicidade das medidas de Haar. Mais precisamente, mostratemos que se $\mu$ e $v$ forem Haar à esquerda (resp. Haar à direita), então existirá uma constante $c>0$ tal que $\mu=c v$. Num caso mais simples, suponhamos que ambas $\mu$ e $v$ sejam Haar à esquerda e à direita. Notemos que este será o caso sempre que $G$ for Abeliano. Fixemos $h \in C_{c}^{+}$tal que $h(x)=h\left(x^{-1}\right)$ - por exemplo, podemos tomar $h(x)=g(x)+g\left(x^{-1}\right)$, onde $g \in C_{c}^{+}$. Nas próximas igualdades, faremos uso sistemático do Teorema de Fubini e das relações de invariância

$$
\int T f d \eta=\int f d \eta, f \in \mathscr{L}^{1}(\eta)
$$

onde $T \in\left\{R_{x}, L_{x}\right\}_{x \in G}$ e $\eta \in\{\mu, v\}$. Tais relações são provadas do mesmo modo que o item (2) do Teorema A.1.

Para qualquer $f \in C_{c}(G)$, a função $F(x, y)=h(y) f(x)$ é integrável sobre $G \times G$ e vale

$$
\begin{aligned}
\int h d v \int f d \mu & =\int h(y) \int f(x) d \mu(x) d v(y) \\
& =\int h(y) \int f(x y) d \mu(x) d v(y) \\
& =\iint h(y) f(x y) d \mu(x) d v(y) \\
& =\iint h\left(x^{-1} y\right) f(y) d \mu(x) d v(y) \\
& =\iint h\left(y^{-1} x\right) f(y) d \mu(x) d v(y) \\
& =\int f(y) \int h\left(y^{-1} x\right) d \mu(x) d v(y) \\
& =\int f(y) \int h(x) d \mu(x) d v(y) \\
& =\int f d v \int h d \mu .
\end{aligned}
$$

Pelo Teorema A.1(3), as integrais de $h$ com respeito a $\mu$ e $v$ são não-negativas, e podemos escrever

$$
\int f d \mu=c \int f d v, f \in C_{c}(G)
$$

onde $c=\int h d \mu / \int h d v>0$. O Teorema da REpresentação de Riesz nos diz então que, para qualquer aberto $U$ de $G, \mu(U)=c v(U)$; e como ambas $\mu$ e $v$ são Radon, a condição de regularidade acarreta $\mu=c v$.

Agora mostraremos o caso geral para medidas de Haar à esquerda. Ainda pelo Teorema de Riesz, será suficiente mostrar que as razões

$$
r_{f}=\frac{\int f d \mu}{\int f d v}
$$


independentes de $f \in C_{c}^{+}$, pois, de acordo com a notação deste mesmo Teorema, se $\eta \in\{\mu, v\}$, então

$$
\eta(U)=\sup \left\{\int f d \eta: f \in \underline{A}(U)\right\}=\sup \left\{\int f d \eta: f \in \underline{A}(U) \cap C_{c}^{+}\right\} ;
$$

de modo que se $R_{f}=R$, para toda $f \in C_{c}^{+}$, então teremos $r v(U)=\mu(U)$ para qualquer aberto $U$ de $G$. Pela regularidade de $\mu$ e $v$, concluímos então que $r v=\mu$.

Queremos mostrar, assim, que $r_{f}=r_{g}$, quaisquer que sejam $f$ e $g$ em $C_{c}^{+}$. De outro modo,

$$
\int f d \mu \int g d v=\int g d \mu \int f d v
$$

Vamos, primeiramente, obter uma versão mais fraca de (A.3): fixadas $f$ e $g$ em $C_{c}^{+}$, procuraremos estimar as diferenças

$$
\left|\int f d \mu \int h d v-\int h d \mu \int f d v\right|
$$

$$
\left|\int f d \mu \int g d v-\int g d \mu \int h d v\right|,
$$

com $h$ variando em $C_{c}^{+}$. O fato é que podemos fazer o caminho inverso e, uma vez provado que as diferenças em (A.4) tornam-se arbitrariamente pequenas para determinadas funções $h \in C_{c}^{+}$, então será possível transportar estas estimativas para $(A .3)$, mostrando que as integrais duplas em $(A .3)$ estão arbitrariamente próximas e, portanto, comutam em $f$ e $g$.

Seja $h \in C_{c}^{+}$. Então

$$
\int f d \mu \int h d v=\iint f(x) h(y) d \mu(x) d v(y)
$$

$\mathrm{e}$

$$
\int h d \mu \int f d v=\iint h(x) f(y) d \mu(x) d v(y) .
$$

As funções $f(x) h(y)$ e $h(x) f(y)$ são obtidas uma da outra a partir da mudança de coordenadas $(x, y) \mapsto(y, x)$. Deste modo, para se obter uma estimativa entre as integrais correspondentes, primeiramente analisemos o efeito de se trocar $x$ por $y$ em $h(x)$ na integral acima. Escolhemos uma função simétrica $h \in C_{c}^{+}$- i.é., $h(x)=h\left(x^{-1}\right), x \in G$ - e, usando propriedades já conhecidas para medidas de Haar à esquerda, obtemos

$$
\iint h(x) f(y) d \mu(x) d v(y)=\iint h\left(y^{-1} x\right) f(y) d \mu(x) d v(y)
$$




$$
\begin{aligned}
& =\iint h\left(x^{-1} y\right) f(y) d \mu(x) d v(y)=\iint h\left(x^{-1} y\right) f(y) d v(y) d \mu(x) \\
& =\iint h(y) f(x y) d v(y) d \mu(x)=\iint h(y) f(x y) d \mu(x) d v(y) .
\end{aligned}
$$

Por outro lado,

$$
\iint f(x) h(y) d \mu(x) d v(y)=\iint h(y) f(y x) d \mu(x) d v(y) .
$$

Assim,

$$
\left|\int f d \mu \int h d v-\int h d \mu \int f d v\right| \leq \iint h(y)|f(y x)-f(x y)| d \mu(x) d v(y),
$$

para qualquer função simétrica $h \in C_{c}^{+}$.

Fixemos uma vizinhança compacta qualquer $V_{0}$ de $e$. Como $f \in C_{c}(G)$, a Proposição A.1 nos garante que existe uma vizinhança $V_{f} \subset V_{0}$ de $e$ tal que

$$
|f(y x)-f(x)|<\varepsilon / 2,|f(x y)-f(y)|<\varepsilon / 2
$$

e como consequência, $|f(y x)-f(y x)|<\varepsilon$, sempre que $y \in V_{f}$ e $x \in G$. Por sua vez, quando $y \in V_{f}$, o suporte de $\varphi_{y}(x)=f(y x)-f(x y)$ deve estar contido no compacto

$$
A=\operatorname{supp}(f) V_{0} \cup V_{0} \operatorname{supp}(f),
$$

pois, caso $y x \in \operatorname{supp}(f)$ ou $x y \in \operatorname{supp}(f)$, então

$$
x \in y^{-1} \operatorname{supp}(f) \cup \operatorname{supp}(f) y^{-1} \subset V_{f} \operatorname{supp}(f) \cup \operatorname{supp}(f) V_{f} \subset A,
$$

e segue da última inclusão que $\varphi_{y}$ se anula fora de $A$. De volta da $(A .5)$, concluímos que se a função simétrica $h \in C_{c}^{+}$for tomada de modo que seu suporte esteja contido em $V_{f}$, então teremos

$$
\left|\int f d \mu \int h d v-\int h d \mu \int f d v\right| \leq \varepsilon \mu(A) \int h d v
$$

Observamos que $A$ não depende de $\varepsilon$, mas somente de $f$, enquanto que $h$ depende de $f$ e $\varepsilon$. O mesmo argumento usado para $f$ aplica-se à $g$, chegando-se à mesma desigualdade obtida em (A.5), com $f$ substituída por $g$. A partir daí, encontramos uma outra vizinhança $V_{g} \subset V_{f}$ de $e$, também simétrica, de modo que $\sup _{x}|g(y x)-g(x y)| \leq \varepsilon$ sempre que $y \in V_{g}$. O suporte de $\phi_{y}(x)=g(y x)-g(x y)$, para $y \in V_{g}$, está contido no compacto

$$
B=\operatorname{supp}(g) V_{0} \cup V_{0} \operatorname{supp}(g)
$$

e, de modo análogo à $(A .6)$, se $\operatorname{supp}(h) \subset V_{g}$, então

$$
\left|\int f d \mu \int f d v-\int h d \mu \int g d v\right| \leq \varepsilon \mu(B) \int h d v,
$$


onde $B$ depende somente de $g$, e $h \in C_{c}^{+}$pode ser tomada como função em comum tanto para (A.6) quanto para (A.7), pois $V_{g} \subset V_{f}$.

Observamos que, pelo Teorema A.1, todas as integrais em questão são não nula; dividindo-se as desigualdades em $(A .6)$ e $(A .7)$ por

$$
\int h d v \int f d v, \int h d v \int g v
$$

respectivamente, obtemos

$$
\left|r_{f}-r_{g}\right| \leq \varepsilon\left(\frac{\mu(A)}{\int f d v}+\frac{\mu(B)}{\int g d v}\right)
$$

Como $\varepsilon$ é arbitrário e independe dos outros termos na desigualdade acima, concluímos que $r_{f}=r_{g}$, como queríamos. Enunciamos, então, o seguinte

Teorema A.3 Se $\mu$ e v forem duas medidas de Haar à esquerda - ou à direita - sobre um grupo topológico compacto, então existirá uma constante $c>0$ tal que $\mu=c v$. 


\section{Referências}

1. Ahlfors, Lars V. - Complex Analysis: an introduction to the theory of analytic functions of one complex variable. Second Edition, McGraw-Hill Book Company.

2. AhLFORS, LARS V. - Lectures on Quasiconformal Mappings, with additional chapters by C. J. Earle and I. Kra, M. Shishikura, J.H. Hubbard. University Lecture Series, volume 38. American Mathematical Society, 2006.

3. Bers, L. AND Royden, H. L. - Holomorphic families of injections. Acta Math. 157(1986), 259-286.

4. Bhatia, R. and Parthasarathy, K. A. - Lectures on functional analysis, I. MacMillan, Delhi. 1977.

5. Bong ChaE, Soo - Holomorphy and Calculus in Normed Spaces. Coleção Pure and Applied Mathematics (Editora MARCEL DEKKER).

6. Bowen, R. - Equilibrium States and the Ergodic Theory of Anosov Diffeomorphisms. Springer Lecture Notes in Mathematics, 470(1975).

7. BowEN, R. - Hausdorff Dimension of quasicircles. Publications Mathématiques de l'IHÉS, Volume 50, Number 1, 11-25.

8. Billingsley, P. - Ergodic Theory and Information. Wiley (1965).

9. Carleson, L. And Gamelin, T. W. - Complex Dynamics. Springer Verlag, 1993.

10. do Carmo, Manfredo P. - Geometria Riemanniana. Projeto Euclides, IMPA, 2005.

11. Chirka, E. M. - On the extension of holomorphic motions. Doklady Akademii Nauk, 397(1): 37-40, 2004.

12. Chirka, E. M. And Rosay, J.P. - On the extension of holomorphic motions. Ann. Pol. Math., 70: 43-77, 1998.

13. Craizer, M. - Teoria Ergódica das Transformações Expansoras. Dissertação de mestrado, IMPA, 1985.

14. Falconer, K. J. - The Geometry of Fractal Sets. Cambridge Tracts in Mathematics, 85.

15. FAlconer, K. - Fractal Geometry - Mathematical Foundations and Applications. John Wiley \& Sons.

16. De Faria, E. And de Melo, W. - Mathematical Tools for One-Dimensional Dynamics. Cambridge studies in advanced mathematics, 115 - Special ICM 2010 edition.

17. Folland, Gerald B. (1999) - Real Analysis, Modern Techniques and Their Applications - Second Edition - Pure and Applied Mathematics: A Wiley-Interscience Series of Texts, Monographs, and Tracts. John Wiley \& Sons, INC.

18. Gamelin, Theodore W. - Complex Analysis. Undergraduate Texts in Mathematics, Springer.

19. Gardiner, F. P. AND LAKIC, N. - Quasiconformal Teichmüller theory. Math. Surveys. Monogr. 76, American Mathematical Society, Providence, R.I., 2000.

20. GARDiner, F. P., JiAnG, Y. AND WANG, Z. - Holomorphic motions and related topics.

21. HAAR, A. - Über die Multiplikation total-additiver Mengefunctionen. Annali Scuola Norm. Sup. Pisa 2 (1933), 429-452.

22. Halmos, Paul R. - Measure Theory, Graduate Texts In Mathematics, Springer-Verlag (1970).

23. Halmos, P. - The Problem of Learning to Teach: The Teaching of Problem Solving. American Mathematical Monthly, 1975.

24. Hewitt, E. And Ross, K. A. - Abstract Harmonic Analysis. Vol. I, Springer-Verlag, Berlin (1963).

25. HÖNIG, C. S. - Aplicações da topologia à análise. Projeto Euclides, IMPA/CNPq (1976).

26. HÖRmAnder, L. - An Introduction to Complex Analysis in Several Complex Variables. Van Nostrand, Princeton, New Jersey (1967).

27. Kato, T. - Perturbation theory of linear operators. Springer, Berlin. 1966. 
28. Khinchine, A. I. - Mathematical Foundations of Information Theory. Dover Publications, New York (1957).

29. Krantz, Steven G. - Function Theory of Several Complex Variables. Pure and Applied Mathematics. A Wiley-Interscience series of texts, tracts and monographs (1982).

30. Lima, Elon L. - Variedades Diferenciáveis. Publicações matemáticas, IMPA, 2007.

31. Mañé, R., Sad, P., And Sullivan, D. - On the dynamics of rational maps. Ann. Ec. Norm. Sup., 96: 193-217, 1983.

32. Martin, M. And England, J. - Mathematical Theory of Entropy. Addison-Wesley (1981).

33. McMullen, Curtis T. - Complex Dynamics and Renormalization. Annals of Mathematics Studies, Princeton University Press.

34. Milnor, J. - Dynamics in One Complex Variable. Third Edtion. Princeton University Press (2006).

35. Munkres, J. R. - Topology, a firt course. Prentice-Hall, Inc., Englewood Cliffs, New Jersey. 1975.

36. Narasimhan, R. - Analysis on Real and Complex Manifolds. Advanced Studies in Pure Mathematics, Masson and Cie, Paris, North-Holland, Amsterdam.

37. Petersen, K. - Ergodic Theory. Cambridge University Press (1983).

38. PARRY, W. AND POLlicotT, M. - Zeta functions and the periodic struture of hyperbolic dynamics. Astérisque, 187-188 (1990). Société Mathématique de France.

39. PRZYTycki, Feliks and URbański, Mariusz. - Conformal Fractals: Ergodic Theory Methods (2009).

40. RAMOS, VANESSA RIBEIRo - Formalismo Termodinâmico e Dimensão de Hausdorff. Dissertação de Mestrado, UFBA.

41. ReEd, M. And Simon, B. (1972) - Methods of Modern Mathematical Physics. I: Functional Analysis Academic Press, INC.

42. Rivera-Letelier, JuAn - Comunicação Pessoal.

43. Royden, H. L. - Real Analysis. Prentice Hall, Englewood Cliffs. Third edition (1988).

44. RuElle, D. - Repellers for real analytic maps. Ergod. Th. and Dynam. Sys.(1982)2, 99-107

45. SlodkowsKi, Z. - Holomorphis motions and polynomial hulls. Proc. Amer. Math. Soc. 111(1991), $347-$ 355.

46. Sullivan, D. - Conformal Dynamical Systems. Geometric Dynamics, 725-752. Springer-Verlag Lecture Notes N. 1007, 1983.

47. Sullivan, D. AND Thurston, W. P. - Extending holomorphic motions. Acta Math. 157(1986), 243-258.

48. WALSh, J. L. - History of the Riemann Mapping Theorem. The American Mathematical Monthly, Vol 80, No. 3 (Mar., 1973), pp. 270-276

49. Walters, P. - An Introduction to Ergodic Theory. Springer (1981).

50. ZinsmeIster, M. - Thermodynamic Formalism and Holomorphic Dynamical Systems. AMS/Société Mathématique de France (2000). 


\title{
Índice Remissivo
}

\author{
$\frac{\partial f}{\partial x}(\xi), 26$ \\ $C(X), 23$ \\ $C(X, Y), 50$ \\ $C^{\gamma}(X ; \mathbf{C}), 86$ \\ $C^{\gamma}(X ; \mathbf{R}), 86$ \\ $C_{0}(X), 14$ \\ $C_{c}(X), 12$ \\ $D^{m} f, 25$ \\ $F(f), 61$ \\ $J(f), 61$ \\ $L(E, F), 25$ \\ $L(X), 83$ \\ $L_{0}, 85$ \\ $P(\phi), 73$ \\ $S_{m} \phi(x), 70$ \\ $\Delta(w, r), 40$ \\ $\Delta^{N}(a, \boldsymbol{\varepsilon}), 105$ \\ $\Delta^{d}(r), 97$ \\ $\Delta_{R}, 37$ \\ $\Delta_{R}^{*}, 97$ \\ $\mathscr{B}, 9$ \\ $\mathscr{F}_{\sigma}, 9$ \\ $\mathscr{G}_{\delta}, 9$ \\ $\mathscr{N}_{T}, 88$ \\ $\mathscr{N}_{g_{0}}, 88$ \\ $\operatorname{dim}_{\mathrm{H}} A, 21$ \\ $\widehat{\mathbf{C}}, 47$ \\ $\frac{d v}{d \mu}, 6$ \\ $\mathrm{C}_{\mathrm{H}}(f), \mathrm{C}_{\mathrm{H}, \delta}(f), 23$ \\ $\lambda$-Lema, 91 \\ $\mu \perp v, 4$ \\ $v \ll \mu, 5$ \\ $\overline{\Delta(w, r)}, 40$ \\ $\partial f(\xi), 26$ \\ C, 1 \\ $\sigma$-álgebra, 3 \\ $\sigma$-compacto, 10 \\ Z, 1 \\ N, 1 \\ $\mathbf{Z}^{+}, 1$ \\ $\mathbf{R}^{+}, 1$
}

Q, 1

R, 1

$\widehat{A}, 29$

$\widehat{d}(x, y), 47$

$\xi \vee \eta, 74$

$d^{m} f, 25$

$d_{\varphi_{z_{0}}}(z, w), 48$

$f^{-1} \mathscr{C}, 73$

$h_{\mu}(\xi), 74$

Álgebra medida, 77

Átomo., 74

Índice de uma curva, 36

Órbita, 56

periódica, 56

aplicação

conforme, 45

diferenciável, 24

Aplicações

biconformes, 46

Aplicações bi-Lipschitz, 22

Autoespaço generalizado, 83

Autovalor, 83

Simples, 83

Autovetor, 83

Bacia de atração, 56

Bisetor, 60

Borelianos, 5

Ciclo, 56

classe $C^{m}, 26$

Componente

Atratora, 62

Parabólica, 62

Periódica, 61

Conforme, Métrica, 62

Conjugação, 56

Conjugação conforme, 56

Conjunto

de Fatou, 61 
de Julia, 61

Nulo, 4

Mensurável, 3

Constante de expansividade, 82

Constante de Hölder, 23

Continuidade absoluta, 5

Convergência uniforme local, 50

Curva, 35

Diferenciável, 35

Diferenciável por pedaços, 35

de Jordan, 53

\section{Derivada, 35}

Derivada de Radon-Nikodym, 6

derivada direcional, 26

Derivada sobre $\widehat{\mathbf{C}}, 48$

derivadas parciais, 27

Diâmetro

de uma coleção de conjuntos, 71

diferencial, 24

diferencial de ordem $m, 25$

Dimensão de Hausdorff, 21

Disco, 50

Disco de Siegel, 62

Distâncias equivalentes, 48

Equilíbrio, 80

Esfera de Riemann, 47

Espectro de $A \in L(X), 83$

Estado de equilíbrio, 80

Exata

Medida, 85

Fórmula de Bowen, 90

Fórmula integral de Cauchy para diferenciais, 38

Família

normal, 51

Família holomorfa, 95

forma matricial, 27

Fréchet, 24

Função

Analítica, 33, 49

G-holomorfa, 43

Localmente limitada, 43

Mensurável, 5

Univalente, 46

Função característica $1_{A}, \chi_{A}, 11$

Função racional hiperbólica, 62

Função simples, 11

Funções

Analíticas reais, 34

Holomorfas, 34

Funcional Positivo, 12

Grau de uma função racional, 59

Grupo

topológico, 109 topológico localmente compacto, 110

Hölder contínua, 23

Haar à direita, 110

Haar à esquerda, 110

Holomorfia de Gâteaux, 43

Integral, 35

Interior de uma curva, 53

Invariante, 55

Probabilidade, 80

Inverso de um conjunto, 109

Isomorfismo, 76

de álgebras medidas, 77

Iterados, 55

método de Newton, 58

Métrica Conforme, 62

Medida

exterior de Hausdorff, 19

medida, 3

Medida com sinal, 4

Medida complexa, 4

Medida de Haar, 110

Medida de Hausdorff, 20

Medida de Radon, 9

Medida exta, 85

Medida exterior métrica, 18

Medida invariante

à direita, 110

à esquerda, 110

Medida positiva, 4

Medidas

Mutuamente singulares, 4

Mixing

Topologicamente, 85

Movimento holomorfo, 91

$\lambda$-Lema, 91

de conjuntos de Julia, 95

Movimento holomorfo normalizado, 92

Multíndice, 40

Multiplicador, 55, 56

Número de cobertura da Haar, 112

Número real

Não-negativo, 1

Não-positivo, 1

Negativo, 1

positivo, 1

Norma

Multíndice, 40

Norma de um polinômio, 30

Norma uniforme, 110

Normalidade, 51

Operador de transferência, 85 
Operadores de translação

à direita, 110

à esquerda, 110

Plano estendido, 47

Polidisco, 40

polinômio homogêneo de grau $m, 29$

Ponto fixo

Atrator, 55

Geometricamente atrator, 55

Indiferente, 55

Repulsor, 55

Superatrator, 55

ponto fixo, 55

Ponto no infinito, 47

Pré-imagem de uma classe, 73

Pressão topológica, 73

Probabilidade

Invariante, 80

Probabilidade exata, 85

Proposição

Aproximação por funções simples, 11

Raio de convergência absoluta, 30

Raio de convergência uniforme, 30

Região, 46

Regular

exteriormente, 9

internamente, 9

regular, 9

Resolvente

Conjunto, 83

Operador, 83
Série de potências, 30

Série de Taylor, 34

Séries de Potências, 40

segmento linear, 24

Simétrico(a)

subconjunto, 109

Soma de Birkhoff, 70

Tamanho

de produtos formais, 70

Teorema

Classficação de componentes periódicas do conjunto de Fatou, 62

de Hurwitz, 53

de Weierstrass, 53

Fórmula de Bowen, 90

da Curva de Jordan, 53

Existência de Medidas de Haar, 116

Regularidade(1), 10

Regularidade(2), 10

Representação de Riesz, 12

Teorema de Hartogs, 39

Topologia

da convergência uniforme local, 50

Topologicamente mixing, 85

Transformação

expansora, 80

Transformação de Cayley, 52

Variação negativa, 4

Variação positiva, 4

Variação total, 7 